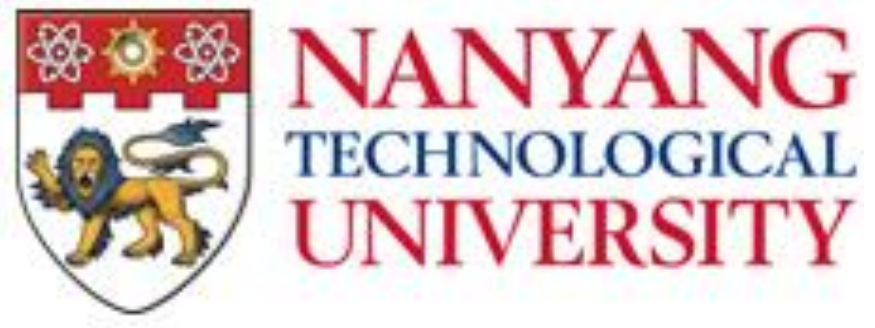

\title{
The Role of Angiopoietin-like 4 in Inflammation
}

\section{Phua Terri}

\author{
School of Biological Sciences \\ A thesis submitted to the Nanyang Technological University in partial fulfillment of the requirement \\ for the degree of Doctor of Philosophy
}

Karolinska Institutet - Department of Microbiology, Tumor and Cell Biology

Work in this thesis was conducted within a joint PhD Programme of the Doctor of Philosophy between the Nanyang Technological University of Singapore, and Karolinska Institutet, Stockholm, Sweden. 
This NTU thesis is an adaptation from my thesis initially published by Karolinska Institutet, with the same title (ISBN 978-91-7676-689-3) and printed by Eprint AB 2017. 
From THE DEPARTMENT OF MICROBIOLOGY, TUMOR AND CELL BIOLOGY

Karolinska Institutet, Stockholm, Sweden

\title{
THE ROLE OF ANGIOPOIETIN-LIKE 4 IN INFLAMMATION
}

\author{
Terri Phua
}

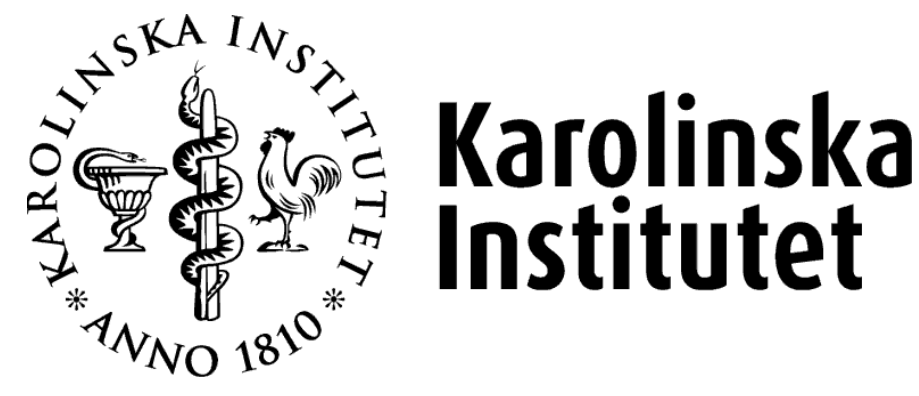

Stockholm 2017 
All previously published papers were reproduced with permission from the publisher. Published by Karolinska Institutet.

Cover illustration depicts The Hive, Nanyang Technological University, Singapore.

Printed by Eprint AB 2017

(C) Terri Phua, 2017

ISBN 978-91-7676-687-3 


\title{
The Role of Angiopoietin-like 4 in Inflammation
}

\author{
THESIS FOR DOCTORAL DEGREE (Ph.D.)
}

By

\section{Terri Phua}

Academic Dissertation

This academic thesis for the Degree of Doctor of Medicine for the NTU-KI Double Degree PhD Program is to be publicly defended in the School of Biological Sciences, College of Science, Nanyang Technological University, Singapore, on $25^{\text {th }}$ May 2017, 14:30 GMT+8.

Principal Supervisor (KI):

Docent Velmurugesan Arulampalam Karolinska Institutet

Department of Microbiology, Tumor and Cell Biology

Principal Supervisor (NTU):

Associate Professor Andrew Nguan Soon Tan Nanyang Technological University

College of Science

School of Biological Sciences

\section{Opponent:}

Associate Professor Feng Rao

Southern University of Science and Technology Department of Biology

\section{Examination Board:}

Docent Benedict Chambers

Karolinska Institutet

Department of Medicine

Associate Professor Valerie Chun Ling Lin Nanyang Technological University

College of Science

School of Biological Sciences

Associate Professor George Wai Cheong Yip National University of Singapore Yong Loo Lin School of Medicine Department of Anatomy 
"It is not the most intellectual of the species that survives; it is not the strongest that survives; but the species that survives is the one that is able best to adapt and adjust to the changing environment in which it finds itself."

Leon C. Megginson. 


\section{ABSTRACT}

Inflammation is a homeostatically fundamental feature of the host defence surveillance system made up of an army of sentinel cells that guard against foreign intrusion. At the onslaught of an attack, primary immune responders (macrophages and neutrophils) secrete a plethora of cytokines and chemokines that attract secondary responders ( $\mathrm{T}$ and $\mathrm{B}$ cells) to the site of inflammation, to eliminate the intrusion and encourage tissue healing. The acute inflammatory stimulation is rapidly attenuated following tissue repair to prevent hypercytokinemia. However, the dysregulation of inflammatory stimulation can also lead to a chronic, erroneous attack on the host immune system, leading to autoimmune diseases and in some cases, cancer initiation. The chronic inflammation theory established by Rudolf Virchow in 1845 is perhaps the first that accurately described the inflammation-induced cancer phenomenon. Since the advancement of technologies, independent researchers have brought forth increasing novel evidence to support the role of inflammation in tumorigenesis. In the compilation of this thesis, we will explore the multi-faceted role of Angiopoietin-like 4 (ANGPTL4) in the regulation of the chemokine landscape during both acute and chronic inflammation.

In Part I, we shall investigate the role of ANGPTL4 in attenuating acute inflammation through the intermediate tristetraprolin (TTP). We show that ANGPTL4 deficiency resulted in an exacerbated inflammatory response in mice subjected to pro-inflammatory stimulation. Confirming the current paradigm, we also reveal that significant microbial divergence was only visible during inflammation and not at steady states. Using immortalized colonic epithelial cells, we connected ANGPTL4 and TTP in the inflammatory signaling axis, and show that ANGPTL4 is able to regulate the acute inflammatory landscape through both TTPdependent and independent signaling pathways.

In Parts II and III, we demonstrate that ANGPTL4 regulates oxidative stress levels in the tumor and tumor microenvironment during chronic inflammation, and induces the epithelialmesenchymal transition (EMT) process. Capitalizing on numerous cultures like A5RT3, MKN78 and HSC5, we first establish that TAK1 protected cells against TGF $\beta$-induced EMT. TAK1 deficiency also promoted Rac1-Nox1 activation, increasing overall ROS production and augmented mesenchymal markers like Snail and E-cadherin. $\mathrm{H}_{2} \mathrm{O}_{2}$ augmented key signaling intermediates which are involved in cellular homeostasis and increased the invasiveness of epithelial cells. In addition, $\mathrm{H}_{2} \mathrm{O}_{2}$ also desensitized cells towards TGF $\beta$ stimulation by decreasing Smad3 and TAK1 expression. To underscore the medical relevance of ANGPTL4 in tumorigenesis, we reveal that ANGPTL4 drives EMT by elevating cellular energy flux through 14-3-3 $\gamma$. In addition, 14-3-3 $\gamma$ promotes the transcription of E-cadherin by stabilizing the binding of Snai1 to its promoter during EMT.

These findings illustrate the importance of ANGPTL4 in regulating the inflammatory landscape during both acute and chronic inflammation, and in tumorigenesis, expanding its role outside of metabolism, insulin resistance, angiogenesis and wound healing. 


\section{LIST OF PUBLICATIONS}

This thesis is based on two publications and one manuscript:

I. Phua T, Sng MK, Tan EHP, Chee DSL, Li Y, Wee JWK, Teo Z, Chan JSK, Lim MMK, Tan CK, Zhu P, Arulampalam V, Tan NS. Angiopoietin-like 4 mediates Colonic Inflammation by Regulating Chemokine Transcript Stability via Tristetraprolin. Sci Rep. 2017; 7:44351.

II. Lam CR, Tan C, Teo Z, Tay CY, Phua T, Wu YL, Cai PQ, Tan LP, Chen X, Zhu $\mathrm{P}$, Tan NS. Loss of TAK1 increases cell traction force in a ROS-dependent manner to drive epithelial-mesenchymal transition of cancer cells. Cell Death Dis. 2013; 4:e848.

III. Teo Z, Sng MK, Chan JSK, Li Y, Li L, Lim MMK, Phua T, Zhu P, Tan NS. Metabolic Reprogramming by Angiopoietin-like 4 is Required for EpithelialMesenchymal Transition. Manuscript under revision.

Other related publications and/or manuscripts are as follows:

IV. Chan JSK, Tan MJ, Sng MK, Teo Z, Phua T, Choo CC, Li L, Zhu P, Tan NS. Cancer-associated fibroblasts enact field cancerization by promoting extratumoral oxidative stress. Cell Death Dis. 2017;8:e2562.

V. Sng MK, Chan JSK, Teo Z, Phua T, Tan EHP, Tan CK, Chen JP, Tong BMK, Tnay YL, Chiba S, Wang XM, Wahli W, Tan NS. Fibroblast PPAR $\beta / \delta$ deficiency causes dermal fibrosis by attenuated LRG1 expression. Submitted Manuscript.

VI. Al-Asmakh M, Anuar F, Phua T, Yip SFM, Kundu P, Zadjali F, Rafter J, Hibberd ML, Fundele R, Tan NS, Parini P, Hedin L, Pettersson S. Commensal microbiota supports placenta development and maternal metabolism. Manuscript. 


\section{CONTENTS}

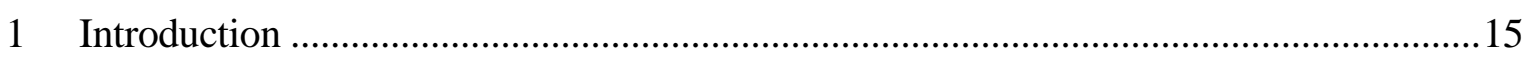

1.1 The Gastrointestinal Tract: Function and Defence .................................................17

1.1.1.1 The Benefits and Hindrance of Host-Microbiota Interactions in the GI tract ..........................................................................19

1.1.1.2 Effects of Development and Lifestyle Adaptations on Microbiota Variability ...................................................................

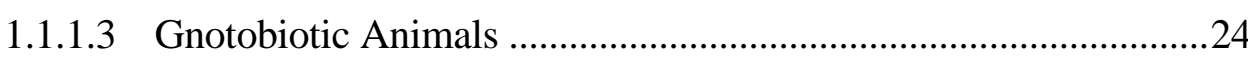

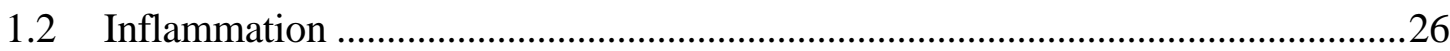

1.2.1 Tristetraprolin (TTP) in Acute Inflammation..............................................27

1.2.1.1 Expression and Regulation of TTP .............................................27

1.2.1.2 mRNA Transcript Stability and its Impact on

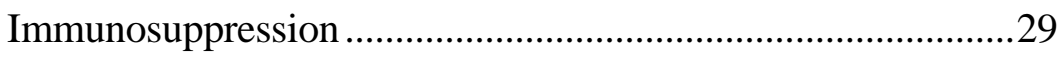

1.2.2 Oxidative Stress in Chronic Inflammation and Cancer ..............................29

1.2.3 TAK1 Expression and Regulation in Chronic Inflammation and

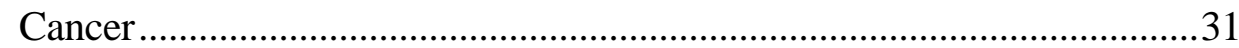

1.3 Angiopoietins and Angiopoietin-like proteins ……………………………..........33

1.3.1 Angiopoietin-like 4 (ANGPTL4) ……………………..............................

1.3.1.1 Expression and Regulation of ANGPTL4 .....................................33

1.3.1.2 ANGPTL4 and Metabolism ..............................................................

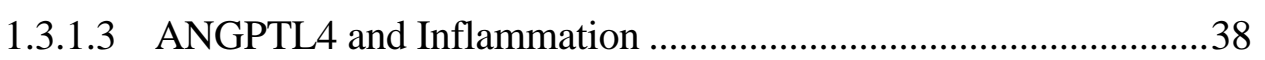

1.3.1.4 ANGPTL4 and Cancer .....................................................................39

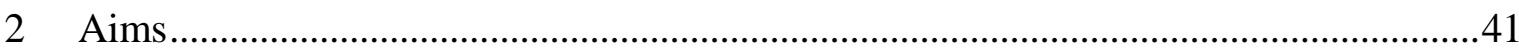

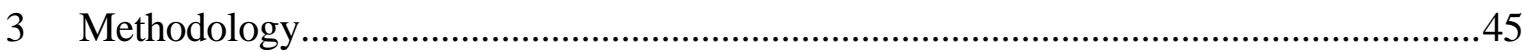

3.1 Methodological Highlights...............................................................................47

3.1.1 Bone Marrow Tranplantation....................................................................4

3.1.2 16S Metagenomics Sequencing ................................................................4

3.1.3 Transwell Migration Assay ......................................................................49

3.1.4 Statistical Analysis ..............................................................................

$4 \quad$ Results and Discussion.........................................................................................

4.1 Angiopoietin-like 4 in Acute Inflammation ...........................................................53

4.2 TAK1 deficiency promotes pro-inflammatory ROS landscape for field

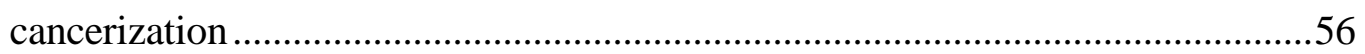

4.3 ANGPTL4 promotes tumor cell migration and EMT ……………………….........58

5 Concluding Perspectives ............................................................................................61

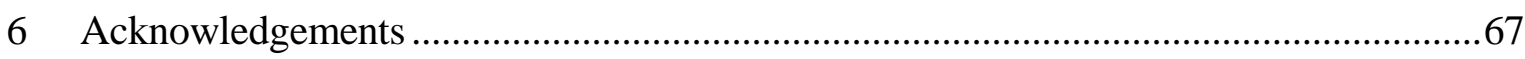

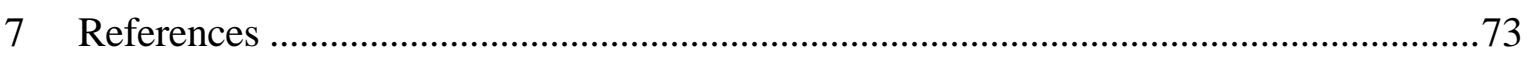




\section{LIST OF ABBREVIATIONS}

185

AKT (PKB)

AMPs

Ang

ANGPTL4

ARES

CANGPTL4

CECS

CCL11

CCL2

CD68

cFos

ChIP

CREB

CRP

CXCL10

CXCL3

CXCL5

DMSO

DSS

ELK1

ERK

fANGPTL4

FIAF

GAPDH

GF

Gl tract

h-cANGPTL4 Ab

HDL

HMP

HRARP

IBD
18S Ribosomal RNA

Protein kinase B

Antimicrobial peptides

Angiopoietin

Angiopoietin-like 4

Adenosine-Uridine Rich Elements

c terminus of ANGPTL4

Colon Epithelial Cells

Chemokine (C-C motif) ligand 11

Chemokine ( $\mathrm{C}-\mathrm{C}$ motif) ligand 2

Cluster of differentiation 68

FBJ murine osteosarcoma viral oncogene homolog

Chromatin immunoprecipitation

CAMP response element-binding protein

C-Reactive Protein

Chemokine (C-X-C motif) ligand 10

Chemokine ( $\mathrm{C}-\mathrm{X}-\mathrm{C}$ motif) ligand 3

Chemokine (C-X-C motif) ligand 5

Dimethyl sulfoxide

Dextran sulfate sodium salt

ETS domain-containing protein

Extracellular signal-regulated kinases

full length fragment of ANGPTL4

Fasting-inducing adipose factor

Glyceraldehyde 3-phosphate dehydrogenase

Germ-free

Gastrointestinal tract

Human cANGPTL4 antibody

High density lipoprotein

Human Microbiome Project

Hepatic Fibrinogen/ Angiopoietin-related Protein

Inflammatory bowel disease 


\begin{tabular}{|c|c|}
\hline iCECS & Immortalized Human Colon Epithelial Cells \\
\hline IECs & Intestinal Epithelial Cells \\
\hline IFN- $\gamma$ & Interferon Gamma \\
\hline IGFR & Insulin-like Growth Factor \\
\hline $\lg G$ & Immunoglobulin G \\
\hline IL-10 & Interleukin 10 \\
\hline IL-17 & Interleukin 17 \\
\hline $\mathrm{IL}-1 \beta$ & Interleukin 1, beta \\
\hline IL-23p19 & Interleukin 23, alpha subunit p19 \\
\hline IL-6 & Interleukin 6 \\
\hline IRS & Insulin Receptor Substrate \\
\hline LCFA & Long chain fatty acids \\
\hline LDL & Low density lipoprotein \\
\hline LPL & Lipoprotein Lipase \\
\hline LPS & Lipopolysaccaharide \\
\hline Ly6G & Lymphocyte antigen 6 complex, locus G \\
\hline MAMP & Microbe-associated Molecular Patterns \\
\hline Muc2 & Mucin-2 protein \\
\hline$n$ & Sample size \\
\hline $\mathrm{NaBu}$ & Sodium butyrate \\
\hline nANGPTL4 & $\mathrm{n}$ terminus of ANGPTL4 \\
\hline NEFA & Non-esterified Fatty Acid \\
\hline NF-KB & NuclearFactor kappa-light-chain-enhancer of activated B cells \\
\hline NGS & Normal goat serum \\
\hline $\mathrm{OA}$ & Oleic acid \\
\hline PBS & Phosphate buffered saline \\
\hline pc-Fos & Phosphorylated FBJ murine osteosarcoma viral oncogene homolog \\
\hline PCR & Polymerase chain reaction \\
\hline pCREB & Phosphorylated cAMP response element-binding protein \\
\hline pELK1 & Phosphorylated ETS domain-containing proteinhomolog \\
\hline PFA & Paraformaldehyde \\
\hline PI3K & Phosphatidylinositol 3-kinase \\
\hline PPAR & Peroxisome proliferator-activated receptor \\
\hline PPRE & PPAR response element \\
\hline
\end{tabular}


PRR

Re-ChIP

Rel Exp

rh-ANGPTL4

S6K

SA

SCFA

SEM

SIRT1

STAT3

TBP

TBS

TBST

TLR4

TNF- $\alpha$

TNFR

TTP

Veh

VLDL

WHO

ZFP36

$\beta$-tubulin
Pattern Recognition Receptors

Re-chromatin immunoprecipitation

Relative expression

Recombinant human c-ANGPTL4

S6 Kinase

Stearic acid

Short chain fatty acids

Standard error of mean

Sirtuin 1

Signal Transducer and Activator of Transcription 3

TATA box binding protein

Tris buffered saline

Tris buffered saline with tween 20

Toll-like receptor 4

Tumor Necrosis Factor Alpha

Tumor necrosis factor receptor

Tristetraprolin

Vehicle control

Very low density lipoprotein

World health organization

Zinc finger protein 36

Beta tubulin 
1 INTRODUCTION 


\subsection{THE GASTROINTESTINAL TRACT: FUNCTION AND DEFENCE}

An estimated $10^{14}$ microorganisms make up the human flora, comprising of more than 10000 bacterial species altogether $[1,2]$. Hence, it is critical that we distinguish and recognize both specific microbes, metabolites and genes in the collection of microbiome that confer health benefits to its host $[3,4]$. To date, the exact composition that makes up the microbiota of a healthy individual remains unknown. Scientists estimate that about $300-1000$ microbial species make up the microflora in the entire gastrointestinal tract. In addition, the lower gastrointestinal tract including both the small and large intestine was found to contain the largest reservoir of intestinal microflora. Microbes residing along the human gastrointestinal tract exhibit habitat-specific traits. To date, studies suggest that more than $99 \%$ of the total microbiota in the gastrointestinal tract consists of anaerobic bacteria, while only those residing in the caecum are believed to be predominantly aerobic [5]. For instance, microbial species representing Bacteroides, Clostridium, Fusobacterium, Eubacterium, Ruminococcus, Peptococcus, Peptostreptococcus, and Bifidobacterium are amongst the most common microbial genera present in the lower gastrointestinal tract of a normal healthy individual [58].

Intestinal epithelial cells (IECs) lining the gastrointestinal tract play an important role as a physical barrier to prevent the invasion of bacteria to the host lamina propria. Hence, the presence of a dynamic and viable intestinal immune system as the first line of defence, allows for a balanced homeostasis between the host and the microbiota community, and in turn, the disregulation of the system, leads to diseases. Although the morphology of the small intestine and the colon differs, the host innate immune approach consists of 2 main strategies: to keep the integrity of the gastrointestinal wall through the continuous production of mucus; and the secretion of antimicrobial peptides (AMPs) by underlying immune and epithelial cells in the lamina propria and submucosa (Figure 1.1). 

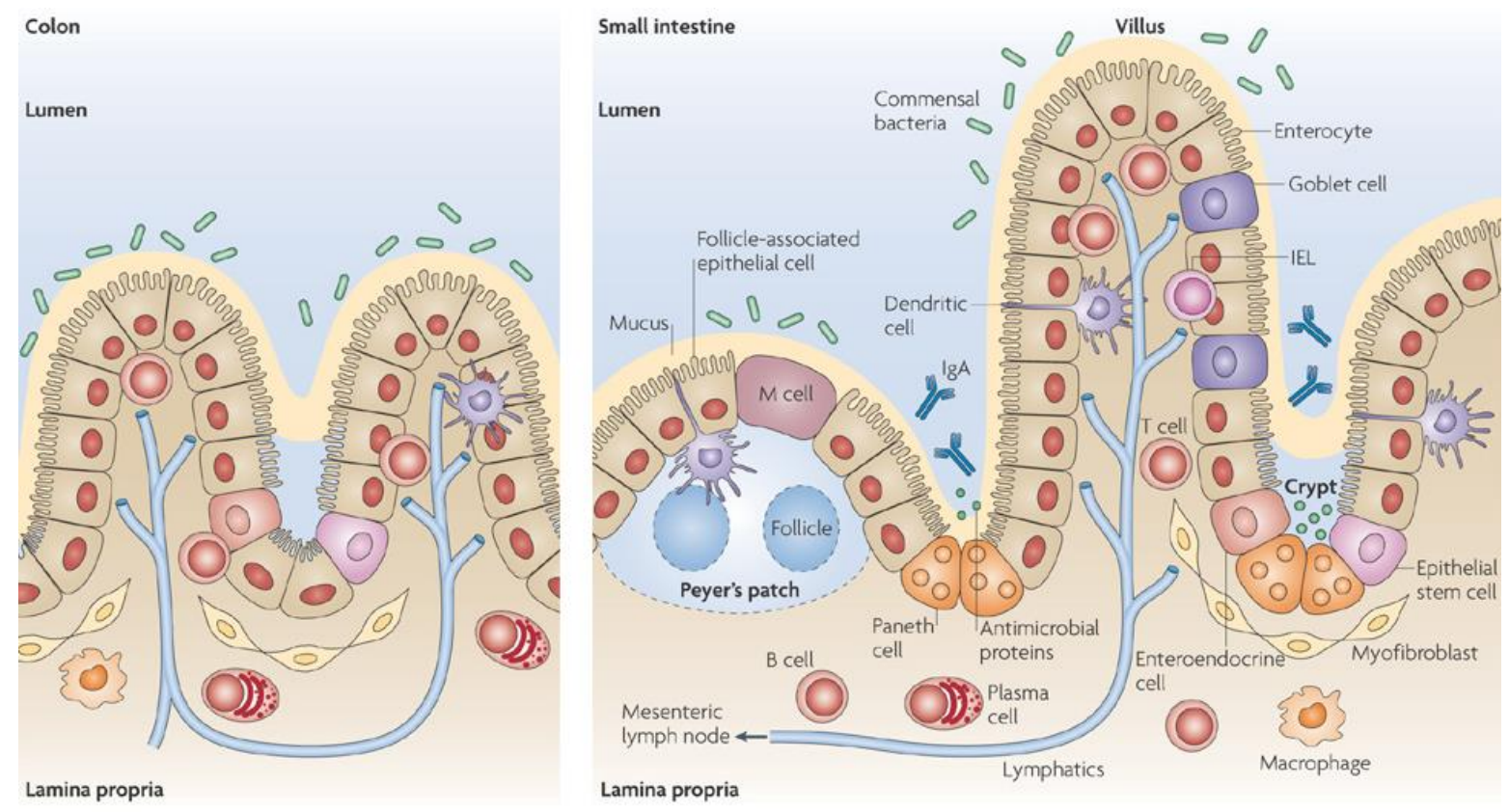

Nature Reviews | Immunology

Figure 1.1 The immune landscape in the gastrointestinal tract. A monolayer of intestinal epithelial cells (IECs) acts as a barrier that partitions the host cells in the lamina propria and the resident microflora in the intestinal lumen. IECs in the colon (colonocytes) and the small intestine (enterocytes) play roles in absorption of water and nutrients respectively. Enteroendocrine and Paneth cells secrete enteric hormones and antimicrobial peptides respectively. Located in proximity with the Paneth cells, epithelial stem cells reside in the crypt regions divide to give daughter cells, which later differentiate into mature IECS as they move towards the luminal surface. Other immune cells like $T$ cells, B cells, dendritic cells and resident macrophages also reside under the lamina propria located close to the IECs. Peyer's patches consisting of lymphatic tissues are also found throughout the ileum, and are primarily responsible for monitoring intestinal bacterial load. Modified and reprinted by permission from Macmillan Publishers Ltd: Nature Reviews Immunology. Abreu MT. Toll-like receptor signalling in the intestinal epithelium: how bacterial recognition shapes intestinal function. Nat Rev Immunol. 10(2):131-144. Copyright 2010.

In the colon, goblet cells are the specialized IECs that keep the resident community of microbiota at bay. Goblet cells residing amongst colonocytes mainly produce mucin-2 protein, which are the building blocks for the mucus matrix [9]. The colon is coated with a double layer of mucus - an inner, stratified layer of mucus in proximity with the epithelial apical surface, as well as an outer, non-attached mucus layer that is in contact with the lumen. Mucin glycans found in the matrix of the outer mucus layer also act as a nutrient supply for resident microbiota. In turn, by-products of microbial fermentation like short chain fatty acids (SCFAs) are absorbed by colonocytes as additional energy source for the host. Because the colon is home to more than $10^{12}$ bacteria per gram of faeces, it becomes of utmost importance that the mucus barrier remains intact to limit events of direct contact between host colonocytes and the gut microbiota. In contrast, the small intestinal mucus layer has been found to be discontinuous because enterocytes are primarily responsible for nutrient absorption and must be in contact with the luminal space [9]. To avert possible assaults, 
Paneth cells located close to the crypt region of the small intestine secrete AMPs like defensins and lectins to form a biochemical barrier in place of the lack of a continuous mucus barrier $[10,11]$ while other host plasma cells secrete immunoglobins (i.e. IgA) [12]. Although once considered to be commensal, the prokaryotic resident microbiota can trigger an inflammatory response and poses severe health risks to its host once the gastrointestinal barrier is breached. Microbes that are capable of degrading mucin-2 and its related glycans can weaken the mucus matrix and hence, reside and grow in proximity with the host epithelial cells.

\subsubsection{The Benefits and Hindrance of Host-Microbiota Interactions in the GI tract}

In humans, only about $85 \%$ of all dietary carbohydrates are absorbed in the proximal small intestine [13, 14]. Indigestible carbohydrates, short chain fatty acids (SCFAs), and polysaccharides representing about $25 \%$ of the total ingested energy enter the distal small intestine and colon $[15,16]$. Without the fermentation action of colonic microbes, this form of energy would have remained inaccessible to the host. The fermentation process of colonic microbes causes the breakdown of large carbohydrates and dietary fibers to give SCFAs like acetate, propionate and butyrate [17], which can then bind to G-protein coupled receptors (GPRs) to regulate a variety of downstream processes $[18,19]$, or be directly utilized as a source of energy by the host (Figure 1.2).

Numerous studies have implicated a continuous interplay between the microbiota residing in the gastrointestinal tract and various host functions. A stable gut microbial landscape resembles a form of defence system because it reduces the risks of pathogenic invasion through competitive exclusion [1]. Commensal microbes have been reported to be involved in the normal development of the gut and also control systemic functions including development, immunity and metabolism in the host $[6,20]$. In addition to secreting digestive enzymes, gut microbes are essential for the production of SCFAs through fermentation of

dietary fibres [21]. SCFAs like acetate, propionate and butyrate also play an integral component of intestinal energy balance and defence [22] (Figure 1.3). 


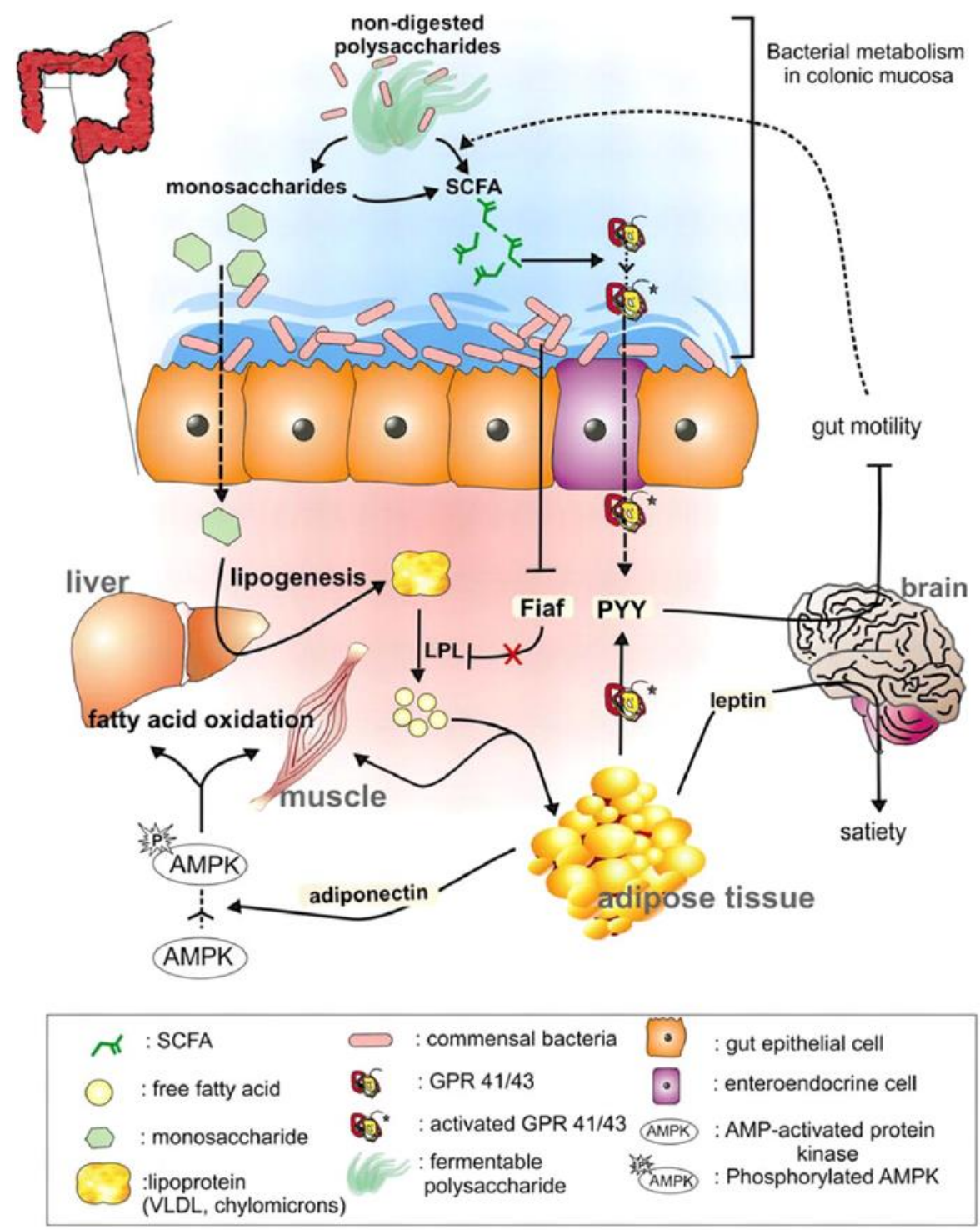

Figure 1.2 Regulation of intestinal microflora on host energy metabolism. If not for intestinal micrbiota, about 30\% worth of the total dietary energy cannot be harvested because the host digestive system cannot break down complex polysaccharides and carbohydrates. The fermentation process by intestinal microbiota gives rise to SCFAs which can be utilized by the host. SCFAs positively regulate GPR $41 / 43$ receptors to increase fermentation. The regulation of ANGPTL4 (FIAF) in intestinal epithelial cells also mediates LPL activity, which alters fat deposition and accumulation in surrounding adipocytes. Reprinted from Nutr Clin Pract., Rosa Krajmalnik-Brown et al, Effects of gut microbes on nutrient absorption and energy regulation, 2012 Apr; 27(2): 201-214, Copyright 2012, with permission from SAGE Publications.

Different members of SCFAs have also been reported to contribute to unique host homeostatic processes that might be both tissue and species specific. The binding affinities between SCFAs are also unique to specific GPRs. For example, it was previously demonstrated that GPR109A is highly selectively for only butyrate [23], while GPR43 binds acetate and propionate over butyrate and GPR41 binds propionate and butyrate over acetate $[24,25]$. Perhaps of all multivariate roles of SCFAs, the anti-inflammatory action of butyrate has most widely been associated with its selectivity and specificity as a potent, non- 
competitive histone deacetylase (HDAC) inhibitor [26]. Butyrate has been reported to mediate the crosstalk between the gut microbiota and host diseases like colitis [27], type II diabetes and obesity [28] through epigenetic regulation and also energy homeostasis through GPR41 [29]. On the other hand, acetate and propionate has been reported to regulate other processes like adipose-specific insulin signaling [30, 31] via GPR43. In a recent study conducted by Ang A et al., acetate was demonstrated to decrease the expression of inflammatory cytokines by increasing p38 phosphorylation and repressing the phosphorylation of AKT and ERK2 in human monocytes [32]. Interestingly, only an increase in p38 and not AKT or ERK2 phosphorylation was observed when mouse monocytes were treated with acetate. Although more studies are needed to show if this acetate/GPR43/41 signaling cascade is unique to monocytes or if it also applies to other cell types or host processes, this monocyte acetate-dependent inflammatory response has been demonstrated to be species-specific.

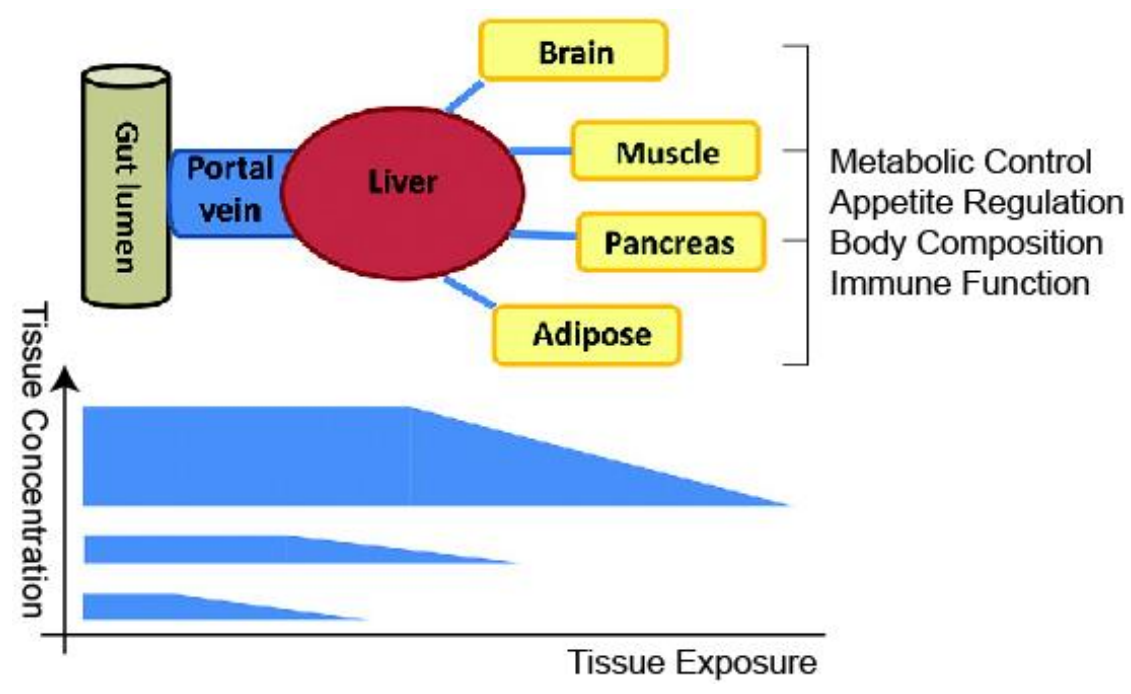

Figure 1.3 The impact and distribution of short chain fatty acids (SCFAs) on host homeostatic processes. The lumen of the gastrointestinal tract is one of the primary sites of SCFA production. As nutrients are absorbed by the epithelial cells and channeled away into the circulation, the concentration of SCFAs reduces. SCFAs are believed to play important roles in regulating host metabolism and immunity. Modified from Gut Microbes, Douglas J. Morrison and Tom Preston. Formation of short chain fatty acids by the gut microbiota and their impact on human metabolism, 2016; 7(3): 189-200. Copyright The Author(s); permitting unrestricted use, distribution and reproduction.

Microbes residing in the colonic mucosa can regulate the levels of epithelial ANGPTL4 [33, 34], while others suggest that ANGPTL4 is a downstream target of GPR 41/43 activation [22, 35]. The regulation of ANGPTL4 alters lipoprotein lipase (LPL) activity in the gastrointestinal tract, directly affecting lipogenesis and the amount of fat deposition and accumulation in circulation, as well as in other storage organs like adipocytes, liver and skeletal muscles. Adipocytes also secrete hormones like adiponectin and leptin in response to changes in SCFA levels. Leptin regulates satiety and appetite in the arcuate nucleus region of the hippocampus $[36,37$ (opposed by ghrelin) while adiponectin activates AMP to AMPK to 
stimulate cellular fatty acid oxidation [38, 39]. Activated by GPR41/43, the secretion of peptide YY (PYY) also reduces peristalsis and gut motility, allowing for increased nutrient absorption [40, 41]. Current studies underscored a significant role for commensal gut microbes in energy regulation, the unwanted enrichment of a subset of microbes can lead to excess energy harvest and storage $[42,43]$ that contribute to the development of various facets of the metabolic syndrome [44, 45]. Current metabolomics findings revealed that obesity is associated with an enrichment of microbes belonging to Actinobacteria and Firmicutes, while decreased fat storage (leanness) has been correlated to the enrichment of Bacteroidetes population [43]. Indeed, genetically obese $(\mathrm{ob} / \mathrm{ob})$ mice on normal chow diet demonstrated an increase in Firmicutes:Bacteroidetes population ratios [46]. However, the cause and effect remains to be determined; it is unclear if the state of obesity selects for Firmicutes population or if a stable population of Firmicutes in the gastrointestinal tract results in inevitable obesity [47].

Despite great mutualistic benefits between microbiota in the gastrointestinal tract and its hosts, it is important to note that these microbes can also become opportunistic pathogens (Figure 1.4). Some famous examples of bacterial-induced inflammation of the gastrointestinal tract (gastroenteritis) include inflammatory bowel disease (IBD). Ulcerative Colitis and Crohn's Disease are well characterized IBDs whereby the commensal gut microbiota in the gastrointestinal tract invade host epithelial cells, causing inflammation predominantly in the ileum and/or the colon which might potentially progress to give colorectal cancer [48]. On the other hand, cholera involves the colonization of Vibrio cholera and infection to the small intestine [49]. A seemingly mild infection by Helicobacter pylori can bring about ulcer formation in the stomach and duodenum. However, H. pylori also secrete a cocktail of enzymes and proteases which further injures epithelial cells, leading to the development of gastric cancer [50]. 


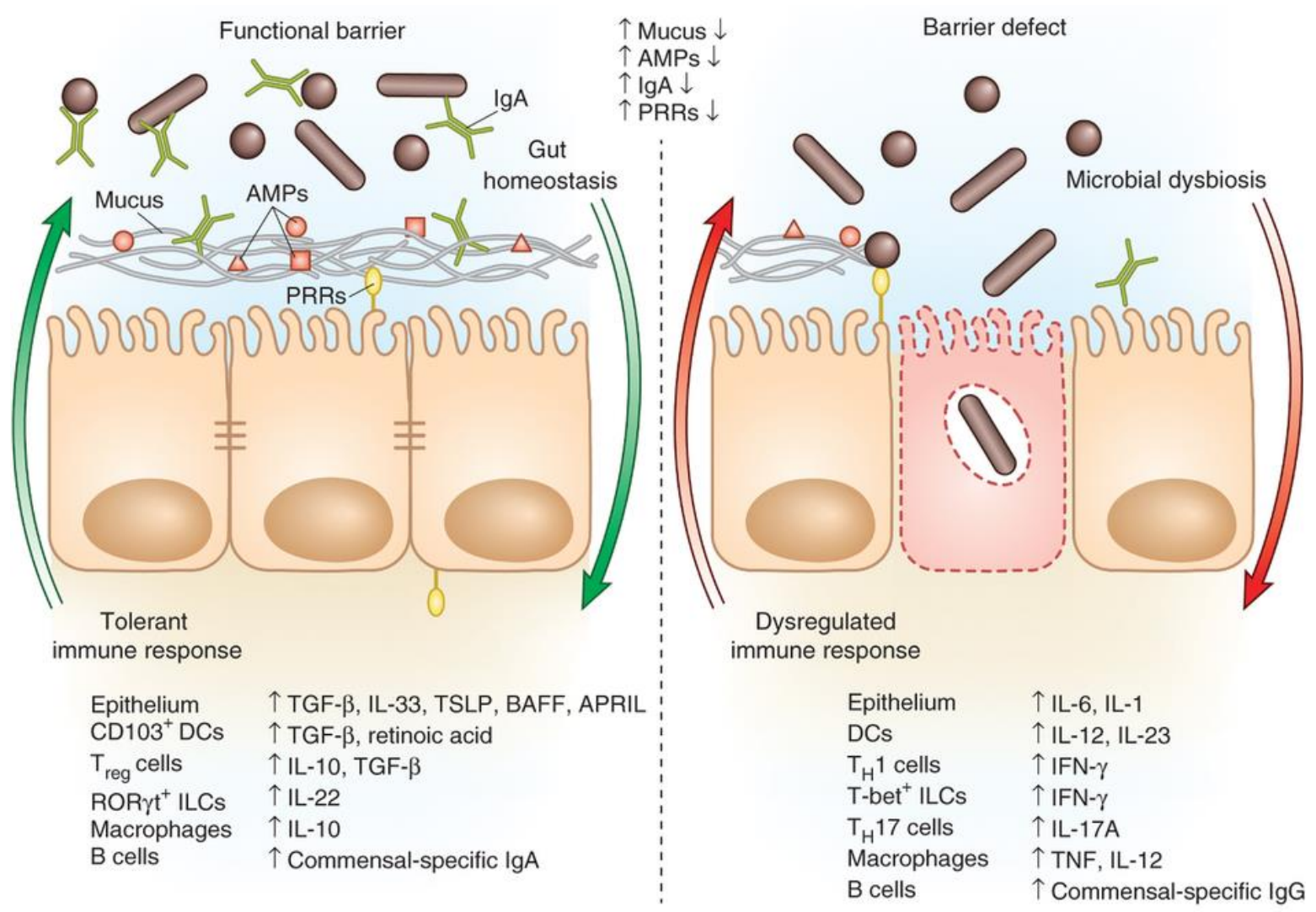

Figure 1.4 An intact and continuous epithelial barrier supports healthy gastrointestinal homeostasis. In a healthy gut environment, cells lining the gastrointestinal wall continuously secrete mucus and anti-microbial peptides (AMPs). Not only does the mucus layer form a physical barrier to keep gut microbiota at bay, it acts as a matrix layer that also allows for a unique habitat for the selection and colonization of commensal gut bacteria. Anti-microbial peptides are secreted by a variety of immune cells located further away in the submucosa and the lamina propria, creating a tolerant immune environment made up of a unique balance of both pro- and anti- inflammatory chemokines. However, a disregulation of mucus or AMP production disrupts healthy gut homeostasis. The lack of a physical compartmentalization of the host from the plethora of gut microbes allows the invasion of opportunistic pathogens. In response to the onslaught, immune cells secrete large amounts of pro-inflammatory chemokines to launch an immune attack against invading pathogens, causing a dysregulated immune envioronment. Reprinted by permission from Macmillan Publishers Ltd: Nature Immunology. Brown EM et al. The role of the immune system in governing host-microbe interactions in the intestine. Nat Immunol. 14(7): 660-667. Copyright 2013.

IECs and a subset of immune cells express protein recognition receptors (PRRs) which aid in monitoring the bacterial load and maintaining healthy intestinal homeostasis [51]. The major PRRs are the Toll-like receptors (TLRs), as well as the nuclear oligomerization domain-like receptors (NOD-like receptors or NLRs). PRRs recognise microbe-associated molecular patterns (MAMPs), which consists of a wide variety of bacterial-related components like peptidoglycans, lipopolysaccharides and microbial RNA/DNA [52]. 


\subsubsection{Effects of Development and Lifestyle Adaptations on Microbiota Variability}

Research suggests changes in the landscape of microbiota in the gastrointestinal tract are also simultaneously observed over the course of development. Currently, 2 distinct milestones during human development - namely, birth and weaning, have observed significant change in the composition of the microbiota in the gastrointestinal tract. While the gastrointestinal tract of the foetus within the womb is thought to be almost sterile, newborns acquire microflora immediately following birth through contact with the mother and also from the surrounding environment. At the outset of birth, infants are believed to first be colonized by facultative anaerobes such as Enterococcus, Streptococcus, Staphylococcus and Enterobacter spp, and thus, creating a reducing environment conducive for the subsequent colonization of strict anaerobic microbes like Bacteroides and Clostridium spp [53, 54]. Although the intestinal microbiota ecosystem is believed to be stabilized up to three years after birth, reports suggest that the various methods of childbirth contributes significantly to the rate of gut microbiota colonization $[55,56]$. During weaning, facultative anaerobes has been observed to give way to obligate anaerobes [2]. As such, the dominant mode of energy synthesis in the gut microbiota switches from aerobic respiration to anaerobic fermentation. Although commensal gut microbiota has been identified to be unique between males and females, little was known about its function on host development. Recent research shows that alterations in the composition of gut microbiota modify the levels of circulating testosterone in males and females to a different extent. This implies that an active crosstalk is present between gut microbiota and host development even in adults [57]. This results in the production of a different landscape of metabolites, which in turn is believed to affect host metabolism.

Consensus accredits the composition of microbiota in the gastrointestinal tract to be predominantly dependent on the lifestyle of the host. For instance, the use of antibiotics during treatment has been observed to alter the microbial ecosystem along the gastrointestinal tract [58]. Recent studies have shown that the reduction of commensal bacteria in the gastrointestinal tract through excessive use of antibiotics in early life increases the risks of developing inflammatory bowel disease and asthma [59]. Strikingly, it has come to attention that the diet could potentially also modify the components of the flora in the gastrointestinal tract. While diet containing high amounts of carbohydrates select for Prevotella species of microbes, diet with high amounts of proteins and fats will favor Bacteroides species. Since the gut microbiota is impermanent and fluid, a change in diet is likely to modify the ecosystem of microbes living in the gastrointestinal tract [60].

\subsubsection{Gnotobiotic Animals}

With the advancement of technology, many groups embarked on independent journeys to study the symbiotic relationship between commensal microbiota and its host. As such, independent groups tried to create the first gnotobiotic model. When the use of antibiotics did 
not successfully eradicate the entire profile of microbes, scientists utilized new technologies that kept animals germ free.

Presumably pioneered by James Reyniers in 1920 [61], germ-free (GF) animals are often used as tools for proof-of-concept trials and were popular in fields like microbiology [43]. However, scientists soon realize that because GF animals had under-developed immune systems, GF mice were more susceptible to pathogenic infections when compared with specific-pathogen-free (SPF) mice that were raised in normal conditions [62]. As interests to understand the plasticity of commensal microbiota grew, attention was drawn to the lower gastrointestinal tract because the ileum and colon harbors the highest density of microbes [58]. To date, evidence suggests that neonatal microbial exposure is crucial in the development of the brain [63] and immune system [64], as well as the membrane barriers of the testis [65] and the blood brain barrier [66]. In addition, recent data indicates that the intestinal microflora might play roles in energy harvest $[33,67]$, and in modulating neuronal signaling cascades that govern behavior and motor control [68].

In recent years, the increasing interests of characterizing GF mice also highlight the importance of PRRs- microbiota associations. GF mice exhibited an under-developed immune system [69]. GF mice lacked immune-associated structures like lymphoid follicles and Peyer's patches in the small intestine [70]. They exhibited an immature and underdeveloped gastrointestinal tract lacking proper mucus formation [71, 72]. In addition, the removal of an important downstream target of TLR signalling, MyD88, saw a decreased response towards inflammatory signals like IL-18 and IL-1 $\beta$ [73], and reduced intestinal lymphocytes [74]. PRR-MAMP complexes also support and boost the production of glycoproteins in the mucus matrix, hence maintaining the integrity of the mucosal barrier [75]. 


\subsection{INFLAMMATION}

Inflammation is a fundamental, homeostatic role of the host defence system. It usually categorised by the cause and mechanism, and can be further divided into the severity (intensity) or eventual outcome. The early surveillance system is made up of an army of immune sentinel cells that alert the host of the intrusion of foreign particles in the body during acute inflammation events. Primary immune cell responders secrete a variety of cytokines and chemokines that attract other specialized immune cells like macrophages, neutrophils, T- and B-cells to combat and eliminate the intrusion - mechanical or microbial and to encourage healing of surrounding tissues. As the wound repair, the inflammation is gradually resolved. Thus, a timed and balanced secretion of both anti- and pro-inflammatory cytokines allow for the rapid initiation and attenuation of inflammatory responses. Chronic inflammation occurs when the inflammation signals continue to persist even after the removal of foreign particles. In these less well-defined events, the sustained secretion of proinflammatory cytokines and chemokines continue to attract immune cells to the presumed site-of-injury, culminating in tissue-damaging cytokine storm, or hypercytokinemia.

In retrospect, the inflammatory bowel disease (IBD) is one of the well-documented microbial-associated, chronic inflammatory events in the gastrointestinal tract. The epithelial cells that line the gastrointestinal tract serve as a physical barrier that separates the host from its surrounding microbiota, nonetheless, they are in close proximity with environmental and dietary carcinogens. It was reported that the increase activation of $\mathrm{CD} 8^{+}$cells coupled with the elevation in ROS production, or KRAS and TP53 mutations aggravate chronic IBDrelated inflammation, supporting a close link to colorectal cancer initiation and progression [76-79]. Recent studies demonstrate that microbial infections also pose significant cancer risks [80]. For instance, Helicobacter pylori (gram negative) and Streptococcus bovis (gram positive) are bacteria that have been associated with gastric and colon cancers. Some viruses have also been shown to cause host DNA mutations by inserting its DNA or RNA into the host cell in attempts for replication. For example, the Epstein-Barr virus (belonging to the family of herpes virus) has been documented to increase the risks to developing lymphoma while certain strains of the human papilloma group of viruses have been linked to the development of cervical cancer. Hepatitis B/C viruses are also correlated to increased risks of developing liver cancer and the human immunodeficiency virus causes the acquired immunedeficiency syndrome (AIDS), which does not cause cancer directly but weakens the immune system of the infected, ultimately elevating the risk of developing other types of cancers or diseases. 


\subsubsection{Tristetraprolin (TTP) in Acute Inflammation}

The role of TTP in acute inflammation was first identified when the embryonically non-lethal mouse deficient of TTP developed progressive manifestations autoimmune, inflammatory responses like erosive arthritis, dermatitis, cachexia and eventually high titers of antinuclear antibodies [81]. Subsequently, it was demonstrated that TTP is involved in the formation of a negative feedback loop responsible of modulating the production of TNF- $\alpha$ by destabilizing its mRNA transcripts [82]. Further studies showed that the removal of the TTP-binding region in the 3' UTR of TNF- $\alpha$ was sufficient to cause inflammatory arthritis [83]. TTP binds to the AREs located at the 3' UTR of TNF- $\alpha$ [84] and initiate deadenlyation [85], initiates deadenlyation to destabilize mRNA transcripts and target them for degradation $[82,86]$. Subsequent findings have identified that TTP also targets other pro-inflammatory and cell cycle proteins [87] like CCL2 [88], c-myc [89], IFN- $\gamma$ [90] and iNOS [91].

The transcription factor NF- $\mathrm{BB}$ is positively regulated by TNF- $\alpha$ and is considered to be a prototypical pro-inflammatory signaling pathway [92]. Functionally active NF- $\kappa \mathrm{B}$ exists as either a hetero- or homo-dimer, made up from the combination of Rel A (p65), Rel B, Rel (c-

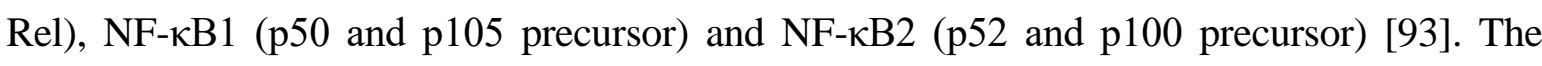
most abundant p65/p50 heterodimer combination is usually sequestered in its inactive state by binding to cytosolic I $\kappa \mathrm{B}$ complexes [94]. Pro-inflammatory stimuli activate the I $\mathrm{B}$ kinase (IKK) complex. Consisting of 3 catalytic subunits, IKK $\alpha, \operatorname{IKK} \beta$ and IKK $\gamma$, IKK

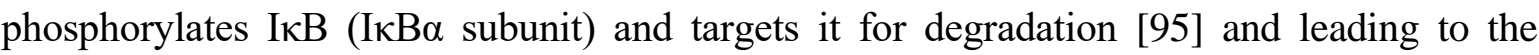
dissociation of the I $\mathrm{KB}-\mathrm{NF}-\kappa \mathrm{B}$ inhibitory complex [96]. This unmasks the nuclear locatlization signal on NF- $\mathrm{KB}$, allowing its nuclear translocation to regulate the transcription of a plethora of pro- and anti-inflammatory target genes engaged in immune modulation [97]. Observations from independent groups indicate that cytosolic TTP is able to bind and prevent the nuclear import of the NF- $\mathrm{BB}$ p65 subunit [98, 99], providing an alternative function of TTP exclusive of its mRNA destabilization activity. Put together, these evidences are consistent with previous postulations [82] that suggest that TTP might have further inhibitory effects on the transcription of a subset of inflammatory-related genes.

\subsubsection{Expression and Regulation of TTP}

Tristetraprolin (TTP), also known as zinc finger protein 36 homolog (ZFP36), Nup475 or GOS24, belongs to a family of proteins containing the $\mathrm{CCCH}(\mathrm{CX} 8 \mathrm{CX} 5 \mathrm{CX} 3 \mathrm{H})$ tandem repeats. TTP was first identified by two independent groups in 1990 using the adipocyte-like, mouse 3T3-L1 cells [100, 101]. TTP has two exons and one intron. Currently, there are four known isoforms of TTP: ZFP36, ZFP36-like 1 (ZFP36L1), ZFP36-like 2 (ZFP36L2) and ZFP36-like 3 (ZFP36L3). Most mammals express three of four TTP isoforms (ZFP36, ZFP36L1 and ZFP36L2) while ZFP36L3 is expressed only in the placenta of rodents [102].

The mouse TTP gene is located on chromosome 7 encoding for a 319-amino acid long protein while the human TTP gene is located on chromosome 19, translating into a 326- 
amino acid long homologue. TTP proteins exist in low levels in non-stimulated cells, predominantly in the nucleus in its unphosphorylated state. Upon stimulation, TTP translocates into the cytoplasm and bind the Adenosine-Uridine rich elements (AU-rich elements, AREs) located at the 3'UTR of target mRNAs [103] (Figure 1.5). The formation of translationally repressed mRNA (translational repression complex) gives rise to two primary forms of mRNA degradation: 3' to 5' decay through exosome complexes or 5' to 3' decay through processing bodies.

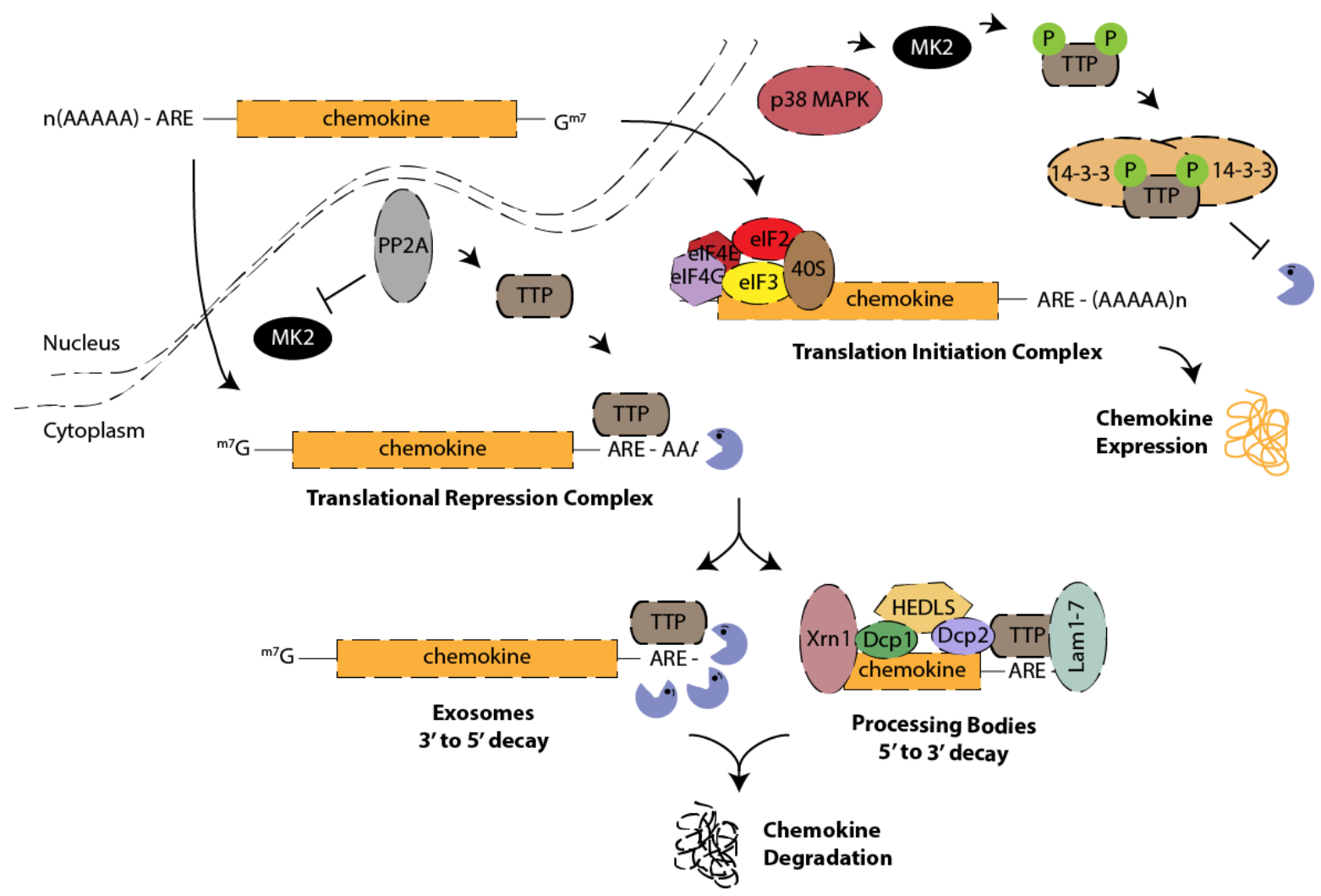

Figure 1.5 Regulation of chemokines by Tristetraprolin. The activity of TTP is an outcome of a balance between PP2A and MK2. p38 MAPK-MK2 dependent signaling causes the phosphorylation of TTP and recruits 14-3-3 complex, sequestering the activity of TTP in the cytosol. On the contrary, PP2A inhibits the action of MK2, resulting in the unphosphorylation of TTP. This allows TTP to recognize and bind the AREs of target mRNAs. This process first triggers deadenylation and the formation of translational repression complexes and the eventual degradation of target chemokines. mRNA degradation happens predominantly through the exosomes $\left(3^{\prime}\right.$ to $5^{\prime}$ decay) and the processing bodies ( $p$-bodies; $5^{\prime}$ to $3^{\prime}$ decay).

Gathering work on TTP revealed that transcription factors and other molecules like SP1, AP2, NF- $\kappa B$, EGR1, TPE1 and ELK1 can transcriptionally regulate the expression of TTP $[104,105]$. TTP was initially identified as an acute-response gene because of its rapid induction towards stimuli like insulin, serum as well as other known mitotic agents like TGF$\alpha$ and p38 MAPK [87, 100, 106-108].

Several serine residues have been found to be phosphorylated in the active form of TTP. Subsequently, TTP has been reported to be stimulated by p38 MAPK and later 
phosphorylated by the mitogen-activated protein kinase-activated protein kinase 2 (MK2) [109] at S60 and S186 in human and S52 and S178 in mouse [87]. However, whether phosphorylated TTP displayed altered ARE-binding capability remains unclear [109]. Interestingly, phosphorylated TTP recruits 14-3-3 adaptor proteins, which reduces the degradation activity of TTP and allowing for downstream chemokine stabilization [110, 111]. The protein phosphotase 2A (PP2A) has been identified to be antagonistic towards MK2 activity, dephosphorylating TTP and causing the degradation of mRNA [110, 112].

\subsubsection{2 mRNA Transcript Stability and its Impact on Immunosuppression}

The balance between target mRNA destabilization and stabilization depends on the ratio of the different cellular regulators present in its environment. In contrast to TTP, the human antigen R (HuR) protein, competes for binding at the ARE of the 3' UTR region of target mRNA transcripts, stabilizing and putting a stop to mRNA degradation [113, 114]. Human $\mathrm{HuR}$ is encoded for by the ELAVL1 gene, is thus, a positive regulator of mRNA stabilization and gene expression [115]. Although much remains to be investigated, it was reported that HuR plays imperative roles in the transcriptional regulation of mRNA transcripts involved in microRNA regulation [116], immune response [117, 118], cancer [119, 120], as well as virus replication [121]. Recently, it was reported that HuR is detrimental in positively regulating the translation of TNF precursors and protein expression into functional TNF- $\alpha$ [122].

The dysregulation or overexpression of pro-inflammatory chemokines and cytokines, i.e. a chemokine storm more commonly known as hypercytokinemia, leads to erroneous damage on the host tissue [123]. Immunosuppressive drugs have been used to rein the hyperactivation of the host immune system. Immunosuppressive drugs are classified into 5 categories: cytostatics, antibodies, anti-immunophilins, glucocorticoids, and other drugs that target active components of the innate immune system. Cytostatics affect the proliferation of B and T cells and largely inhibit cell division [124]. Antibodies are sometimes used as quick-response agents to quench the immune response $[125,126]$. The use of drugs against immunophilins are targeted at the inhibition of prolyl isomerise to prevent the interconversion between the cis and trans position of peptidyl-prolyl groups [127]. Glucocorticoids are arguably the most extensively used immunosuppressant to date [128]. Glucocorticoids inhibit a wide assortment of interleukins [129] and chemokines [128, 130], as well as decreasing eicosanoid and cyclooxygenase (COX-1/2) synthesis and expression [131, 132]. Interestingly, glucocorticoids have been observed to positively regulate MKP-1 (also recognised as DUSP1) [133-135], which has shown to increase the activity of TTP-dependent destabilization of pro-inflammatory cytokines [136-138].

\subsubsection{Oxidative Stress in Chronic Inflammation and Cancer}

The rapid development and advancement of technology over the last century fueled novel research techniques that supported Rudolf Virchow's 'chronic irritation theory'. In 1845, Virchow coined the term leukemia when he reported significant elevation of white blood 
cells in patients. Subsequently, he demonstrated that normal cells can become neoplastic to give rise to cancers [139], and described the cancer initiation and propagation as a result of tissues under sources of severe irritation that spread through a liquid medium. Virchow also described that cancers were intrinsically related to white blood cells which were the original cause of this irritation, an observable fact we now relate to as tumor-associated macrophages during inflammation [140-142]. In subsequent breakthrough findings, Katsusaburo Yamagiwa et al demonstrated the role of chemical carcinogens in cancer [143] while Francis Peyton Rous et al established the existence of cancer-causing viruses [144]. Scientists then discovered a cluster of genes that regulated cellular growth (proto-oncogenes that are mutated to give oncogenes) as well as the cellular DNA repair mechanism (tumor suppressor genes). Researchers also hypothesized the two-hit hypothesis (also known as the Knudson hypothesis), which suggests that multiple hits were required to induce cancer. In this respect, the term field cancerization becomes an extension of the two-hit hypothesis because an earlier hit increases the predisposition of the cells in the cancer vicinity to developing a larger tumor.

Numerous mechanisms by which chemical carcinogens caused tumorigenesis have been proposed [145, 146]. One common underlying theme is the production of free radicals, such as reactive oxygen (ROS) and reactive nitrogen species (RNS) [147]. Because of their highly reactive nature, the balance between the production of ROS and their elimination by antioxidants is important for tissue homeostasis. It is now well-established that low concentrations of these reactive species served as signalling mediators [148, 149]. However, ROS imbalance or the loss of homeostatic control in a continued oxidative microenvironment can either result in cell death caused by oxidative catastrophe or contribute to carcinogenesis, which are tissue- and context-dependent. Environmental factors like heightened background radiation and pollutant levels in cities, or increase intake of drugs and xenobiotics also increases the exposure to exogenous ROS/RNS. Endogenous sources of ROS/RNS are generated by essential biological processes such as respiration and inflammation. During respiration, free electrons are transported from one membrane-bound donor protein to its successive recipients arranged in progressively lower energy levels along the electron transport chain to create a proton gradient during mitochondrial respiration and ATP synthesis. Under normal homeostatic conditions, oxygen is completely reduced to water at the end of the respiratory cycle. However, incomplete reduction of molecular oxygen leads to the formation of the superoxide radical $\left(\mathrm{O}_{2}{ }^{-}\right)$, a regular precursor of most other ROS. In its protonated form, hydroperoxyl $\left(\mathrm{HO}_{2}^{-}\right)$has been shown to inactivate enzymes and accelerate lipid peroxidation $[150,151]$. The dismutation of $\mathrm{O}_{2}{ }^{-}$gives rise to hydrogen peroxide $\left(\mathrm{H}_{2} \mathrm{O}_{2}\right)$, and may further be partially reduced to give the hydroxyl radical $\left(\mathrm{OH}^{-}\right)$.

In normal cells, oxidative stress conjures protective antioxidant response from tissues to regain homeostasis. Antioxidant mechanisms involving enzymatic and non-enzymatic pathways are in place to prevent ROS accumulation. Notably, antioxidant molecules like vitamins $\mathrm{A}$ and $\mathrm{E}$, glutathione, bilirubin and co-enzyme $\mathrm{Q}$ assist in quenching ROS in the body. Enzymes belonging to the family of superoxide dismutases (SODs) or catalyses can be site-specific and help catalyze the reaction of superoxides to the less harmful hydrogen 
peroxides and oxygen. SODs contain different cationic metallic reactive centres and are located in the cytoplasm (SOD1), mitochondria (SOD2) and even in the extracellular matrix (SOD3). Catalyses are concentrated primarily in peroxisomes that are located in proximity to mitochondria and glutathione peroxidises rely on its reactive sulphur centre to reduce ROS.

Perturbations to the balance of antioxidant and oxidants, resulting in accumulation of ROS/RNS causes oxidative stress, and have been documented to cause breakages in the DNA double helixes, mismatches in the complementary DNA base-pairings and also base transversions [152]. Many studies also show that the build-up of ROS promotes disease pathogenesis by acting as a precursor to fuel chronic inflammation and malignancy. Metabolically active organs like the brain and the liver are also highly susceptible to ROS accumulation. ROS elevation in the brain has been reported to increase risks of Alzheimer's disease [153, 154] while chronic hepatitis and liver fibrosis have been attributed to the accumulation of ROS in the liver $[155,156]$. ROS has been reported to cause mutations in critical cell cycle proteins like checkpoint kinases $1 / 2$ (Chk1 and Chk2) and ataxia telangiectasia (ATM), as well as induce G2-M phase cell cycle arrest [157]. Others found that the increase in ROS $[158,159]$ in the hypoxic tumor environment stimulated the subsequent augmentation of TNF- $\alpha$, COX-2 [160], NF- $\kappa B$ [161], STAT3 [162] and JNK/p38 MAPK [163] activity, which brought about further increase in IL-6, TNF- $\alpha$ and BCL-X levels, hence promoting tumor cell survival, migration and metastasis.

\subsubsection{TAK1 Expression and Regulation in Chronic Inflammation and Cancer}

The transcription factors nuclear factor- $\kappa \mathrm{B}(\mathrm{NF}-\kappa \mathrm{B})$ and activating protein-1 (AP-1) are critical regulators of stress responses, immunity, inflammation and cancer. A large variety of cellular stimuli utilize these signaling pathways through a common upstream kinase transforming growth factor- $\beta$-activated kinase 1 (TAK1). TAK1 belongs to the family of mitogen-activated protein kinase kinase kinase (MAPKKK) class of threonine/serine kinases and is encoded for by the MAP3K7 gene on chromosome 6. TAK1 has been found to complex with both Tab1 and Tab2 proteins in the cytosol to facilitate its downstream signaling. The association with Tab1 is believed to activate the TAK1 kinase activity [164] while Tab2 assists in the binding with the TRAF6 complex [165]. TAK1 deficiency in mouse resulted in embryonically lethal phenotypes because of the inability to undergo important processes like angiogenesis and vascularization during development [166].

TAK1 has been well characterized in recent literature in playing pivotal roles in development and innate inflammation and has been found to respond to a plethora of inflammatory stimulation. Bacterial LPS, inflammatory cytokines and TLRs have been reported to stimulate TAK1 activity [167-171]. Although non-canonical, it was also reported that TGF $\beta$ can stimulate TAK1 activity through Smad3 independent pathways [172]. TAK1 was also found to phosphorylate other downstream MAP kinases, culminating in the activation of transcription factors like p38 MAPK, NF- $\mathrm{BB}$ and AP-1 that regulates pro-inflammatory genes 
[173-176]. Importantly, evidence have showed that TAK1 deficiency decreases cJun activation and promotes ROS production and inflammation in the skin [177]. Others demonstrate that TAK1 phosphorylates MAPKs [178] and increases TNF- $\alpha$ signaling [179, $180]$, resulting in the increased production of COX-2 and ROS. 


\subsection{ANGIOPOIETINS AND ANGIOPOIETIN-LIKE PROTEINS}

There are 4 members that make up the angiopoietin family of proteins (Ang). While proangiogenic Ang1 was found to bind its cognate receptor Tie1 and Tie2 to promote angiogenesis and downstream vessel remodelling [181], it was later reported that Ang2 antagonises this effect by competitive binding to Tie2 [182]. Angiopoietins were first identified to be mediators of blood vessels formation during embryonic and postnatal development, and also for the assemblage of the vascular endothelial wall [183, 184].

The Ang family of proteins share structural and sequence homology to the Angiopoietin-like family of proteins (ANGPTL) because both Ang and ANGPTL family of proteins are characterized by a coiled-coil $\mathrm{N}$-terminal domain with a fibrinogen-like $\mathrm{C}$-terminal domain [185-187]. ANGPTL represents a family of 8 proteins. But unlike their cousins, ANGPTL do not bind Tie proteins to mediate downstream responses (18206803). To date, ANGPTL remain as orphan ligands with little knowledge on their direct binding partners. Thus far, studies have observed that ANGPTL2, ANGPTL3, ANGPTL4, ANGPTL5, ANGPTL7 are involved in hemapoietic stem cell maintenance and regulation while ANGPTL3, ANGPTL4, ANGPTL6 and ANGPTL8 have been reported to mediate glucose and lipid metabolism [188190]. Recent studies have also detailed the multifaceted role of ANGPTL in inflammation and cancer progression and metastasis [191-193].

\subsubsection{Angiopoietin-like 4 (ANGPTL4)}

ANGPTL4 is perhaps one of the most important members of the ANGPTLs because it mediates a broad variety of processes. Studies show that ANGPTL4 is involved in a wide assortment of pathways ranging from energy homeostasis, wound repair, inflammation and cancer [194-197]. Emerging evidence indicates that ANGPTL4 alters endothelial barrier integrity and is involved in cancer cell migration and metastasis [198]. Lately, the dysregulation of ANGPTL4 has also been attributed to complications associated with the metabolic syndrome.

\subsubsection{Expression and Regulation of ANGPTL4}

ANGPTL4 is a secretory adipokine previously known as fasting induced adipose factor (FIAF), hepatic fibrinogen/angiopoietin-related protein (HRARP) and peroxisome proliferator-activated receptor gamma angiopoietin-related protein (PGAR). The ANGPTL4 protein is encoded for by the ANGPTL4 gene, a $11.09 \mathrm{~kb}$ gene located on human chromosome 19, and translated into a 406-amino acid long protein. On the other hand, the mouse ANGPTL4 gene is $6.6 \mathrm{~kb}$ on chromosome 17 encoding for a 410-amino acid ANGPTL4 protein. The molecular weight of full length ANGPTL4 (fANGPTL4) in both mice and humans remains contentious, ranging between 45 to $65 \mathrm{kDa}$ due to posttranscriptional modifications such as glycosylation (Figure 1.6). 


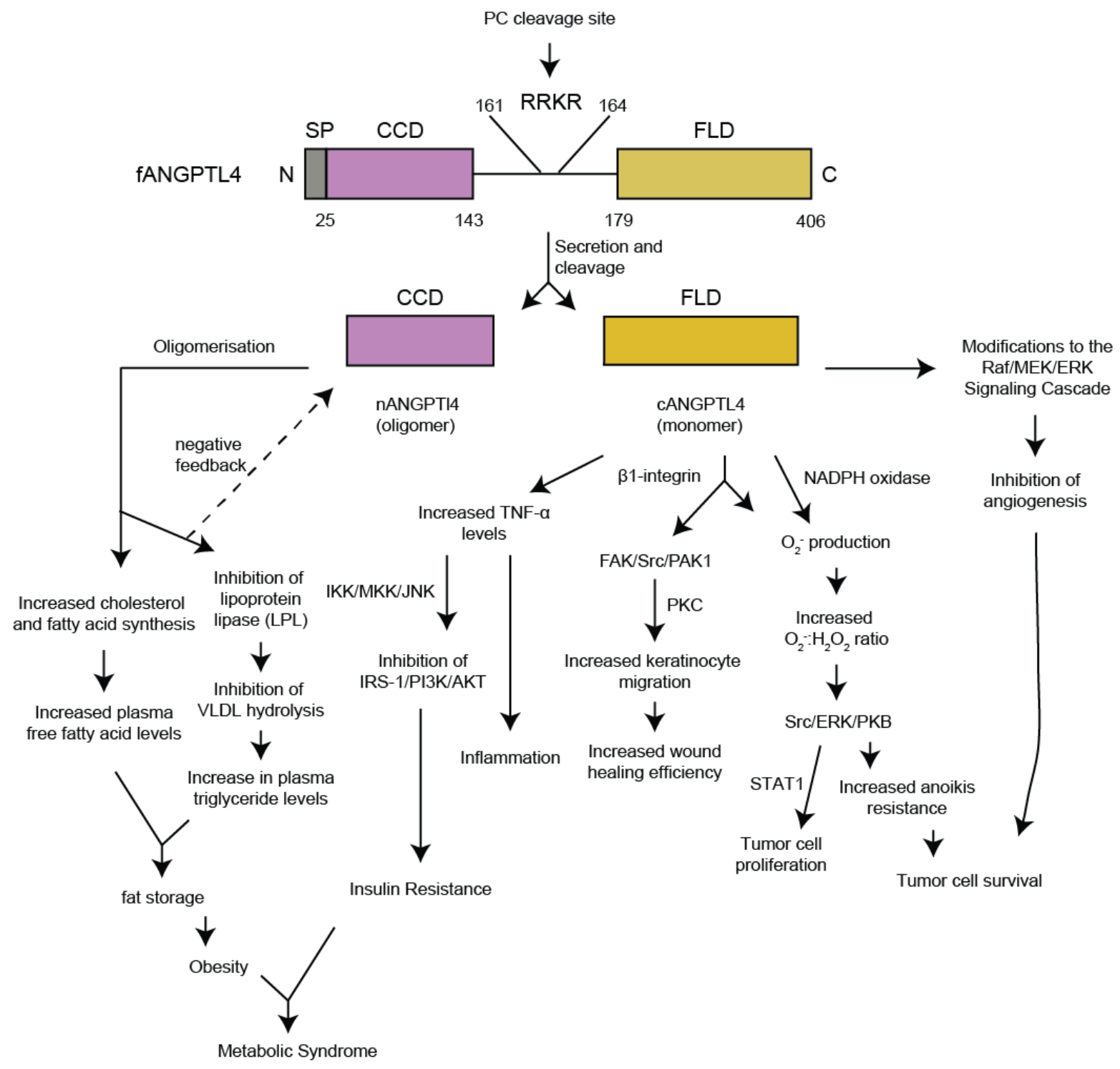

Figure 1.6 Regulation and function of Angiopoietin-like 4 (ANGPTL4). Proprotein convertases like furin have been reported to recognize a conserved sequence -RRKR- of non-functional full length ANGPTL4 (fANGPTL4) to give an N-terminal coiled-coil fragment (nANGPTL4, $26 \mathrm{kDa}$ ) as well as a Cterminal fibrinogen-like fragment (cANGPTL4, 35-47 kDa). Functional nANGPTL4 oligomerizes into dimmers or tetramers, and has been found to inhibit lipoprotein lipase (LPL), primarily controlling downstream fat metabolism and storage. On the other hand, functional cANGPTL4 exists as monomers. Recent findings direct the function of CANGPTL4 to play regulatory roles in inflammation, as well as tumor cell survival and proliferation. Modified and reprinted from Mol Cancer Res, 2012, 10, 677-688, Tan MJ et al, Emerging Roles of Angiopoietin-like 4 in Human Cancer, with permission from AACR.

Once secreted, fANGPTL4 is cleaved by proprotein convertases at the -RRXR- consensus region to give a $25 \mathrm{kDa}$ N-terminus coiled-coil fragment (nANGPTL4) and a 25-35 kDa Cterminus fibrinogen-like fragment (cANGPTL4). To date, the proteolytic cleavage mechanism of fANGPTL4 remains to be elucidated. In vitro studies have shown that proprotein convertases like furin and paired basic amino acid-cleaving enzyme 4 can cleave fANGPTL4 at its consensus site [199]. However, little is known if these proprotein convertases are indeed responsible for the in vivo processing of secreted fANGPTL4, or if the 
expression of the different classes of proprotein convertases correlates to the presence of different ANGPTL4 fragments. Unlike cANGPTL4 which exists as a monomer, disulfide bond formation at the highly conserved 76 and 80 residues of the $\mathrm{N}$-terminus coiled-coil domain allows fANGPTL4 to oligomerize and exist either as a tetramer or a dimer [200]. Likewise, the nANGPTL4 fragment also oligomerizes after cleavage and regulates lipid metabolism via its interaction with lipoprotein lipase.

During hypoxia, the expression of hypoxia-inducing factor $1 \alpha$ (HIF-1 $\alpha)$ is up-regulated, which mediated downstream ANGPTL4 expression [201, 202]. ANGPTL4 is also a direct target gene of the nuclear receptor PPAR (peroxisome proliferator-activated receptor) and functional PPAR response element (PPRE) resided in the intron 3 of the ANGPTL4 gene [203]. HIF-1 $\alpha$ and PPAR were also found to synergistically up-regulate the expression of ANGPTL4 [204]. Other reports indicated that a variety of growth factors and stimuli like the toll-like receptors (TLRs), transforming growth factor beta (TGF $\beta$ ), glucocorticoid receptor (GR), the metabolites from gut microbes and even the circadian rhythm signals ARNT2/SIM1 also regulate the expression of ANGPTL4 [188, 205-210].

fANGPTL4 is readily detected in hepatocytes, adipocytes and macrophages, as well as a lower level in other tissues such as the skin, kidneys and intestines [188] while cANGPTL4 and nANGPTL4 fragments are detected in the liver [203, 211]. The nANGPTL4 associates with lipoproteins [212] and also inhibits the activity of lipoprotein lipases (LPL). This interaction is dependent on the oligomerization of nANGPTL4 fragments in order to effectively inhibit LPL and participate in lipid metabolism [213] (Figure 1.7). Hence, the function of nANGPTL4 hinges on its ability to form active three-dimensional structures which are functional. In contrast, the cANGPTL4 fragment exists as a monomer. cANGPTL4 associates with specific extracellular matrix proteins, $\beta 1$ and $\beta 5$ integrin molecules [214]. In wound healing, cANGPTL4 facilitates keratinocyte migration through the FAK/Src/PAK-1 cascade [215]. The cANGPTL4 also suppresses angiogenesis through the inhibition of the Raf/MEK/ERK pathway [216]. Furthermore, cANGPTL4 also increases the $\mathrm{O}_{2}{ }^{-}: \mathrm{H}_{2} \mathrm{O}_{2}$ ratio, promoting tumor cell survival and proliferation [197]. Put together, these evidences suggest that the expression of specific ANGPTL4 fragments is tissue/organ dependent, and that the various ANGPTL4 fragments play distinct cellular functions. 


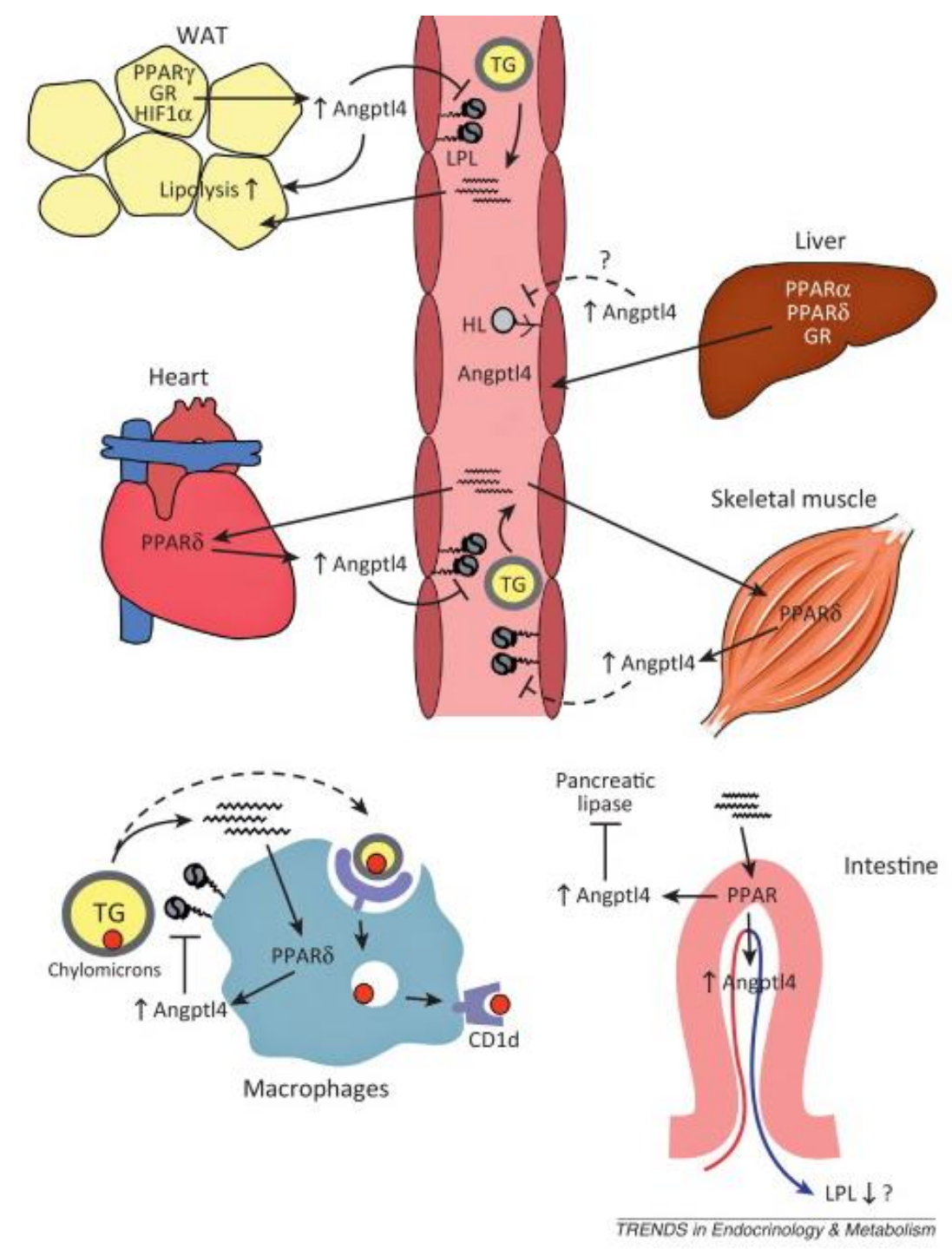

Figure 1.7 The paracrine effects of Angiopoietin-like 4 (ANGPTL4) in lipid metabolism. ANGPTL4 is chiefly expressed in the liver, white adipose tissue (WAT), skeletal muscle but is recently reported to be also expressed in macrophages, heart as well as enteroendocrine cells in the intestine. The function of ANGPTL4 in lipid metabolism is tissue specific, and is primarily under the regulation of peroxisome proliferator-activated receptors (PPARs) and glucocorticoid receptors (GRs). In WAT, increased ANGPTL4 stimulation inhibits lipoprotein lipases and increases lipolysis of accumulated triglycerides. Because the liver does not produce LPL, ANGPTL4 is secreted into the circulation and targeting peripheral LPL activity. In the muscle, heart and in macrophages and intestinal enteroendocrine cells, positive stimulation by fatty acids increases ANGPTL4 levels, bringing about an inhibition of local LPL activity. Reprinted from Trends in Endocrinology \& Metabolism, 25/3, Dijk W et al, Regulation of lipoprotein lipase by Angpt|4, Pages No. 146-155, Copyright 2014, with permission from Elsevier.

Up till now, there is no known protein crystal structure of fANGPTL4, cANGPTL4 or nANGPTL4. Current knowledge of the different roles of ANGPTL4 fragments were elucidated via a combination of biochemical analysis and ANGPTL4-knockout animal model. ANGPTL4 has been implicated to play a role in tumorigenesis, however its effect remained controversial. Although the precise reason for the controversy is unclear, it is conceivable that different ANGPTL4 fragments may be involved, as these fragments have 
been reported to stimulate different signaling cascades Thus, more effort is required to elucidate the structures and functions of the different ANGPTL4 fragments.

\subsubsection{ANGPTL4 and Metabolism}

The oligomerized nANGPTL4 inhibits LPL and regulates lipid metabolism (Figure 1.8). LPLs are found at the luminal surface of capillary endothelial cells and are regulated by hormones like insulin, glucagon and adrenaline. LPL homodimers hydrolyses plasma triglycerides found in VLDL into intermediate-density lipoproteins (IDL) and eventually into free fatty acids (FFA), allowing the subsequent uptake of fatty acids into the liver, adipocytes and macrophages for storage purposes. ANGPTL4 mediates the rate of plasma triglyceride clearance. fANGPTL4 and nANGPTL4 oligomers cause the dissociation and subsequent inactivation of LPL dimers, resulting in the inhibition of triglyceride hydrolysis. Furthermore, it was observed that ANGPTL4 further exacerbates the inhibition of triglyceride hydrolysis by targeting LPLs for proteolysis by proprotein convertases [199]. However, studies also showed that increasing circulating ANGPTL4 further increases plasma FFA levels through increasing cholesterol and fatty acid synthesis [211, 217], suggesting a negative feedback mechanism on plasma LPL inhibition. The over-expression of ANGPTL4 was also demonstrated to increase levels of nonesterified fatty acids (NEFA) in adipocytes [212] and cause hypertriglyceridemia in mice $[212,218]$.

Apart from lipid metabolism, ANGPTL4 was recently found to be involved in glucose metabolism. ANGPTL4 has been shown to reverse glucose insensitivity in diabetic mice. ANGPTL4 lowers hyperglycemia primarily through augmenting insulin-dependent inhibition of gluconeogenesis and impeding glucose synthesis in the liver [219]. In adipocytes, insulin down-regulates ANGPTL4 levels through the PI3K/FOXO1 signalling cascade [220]. 


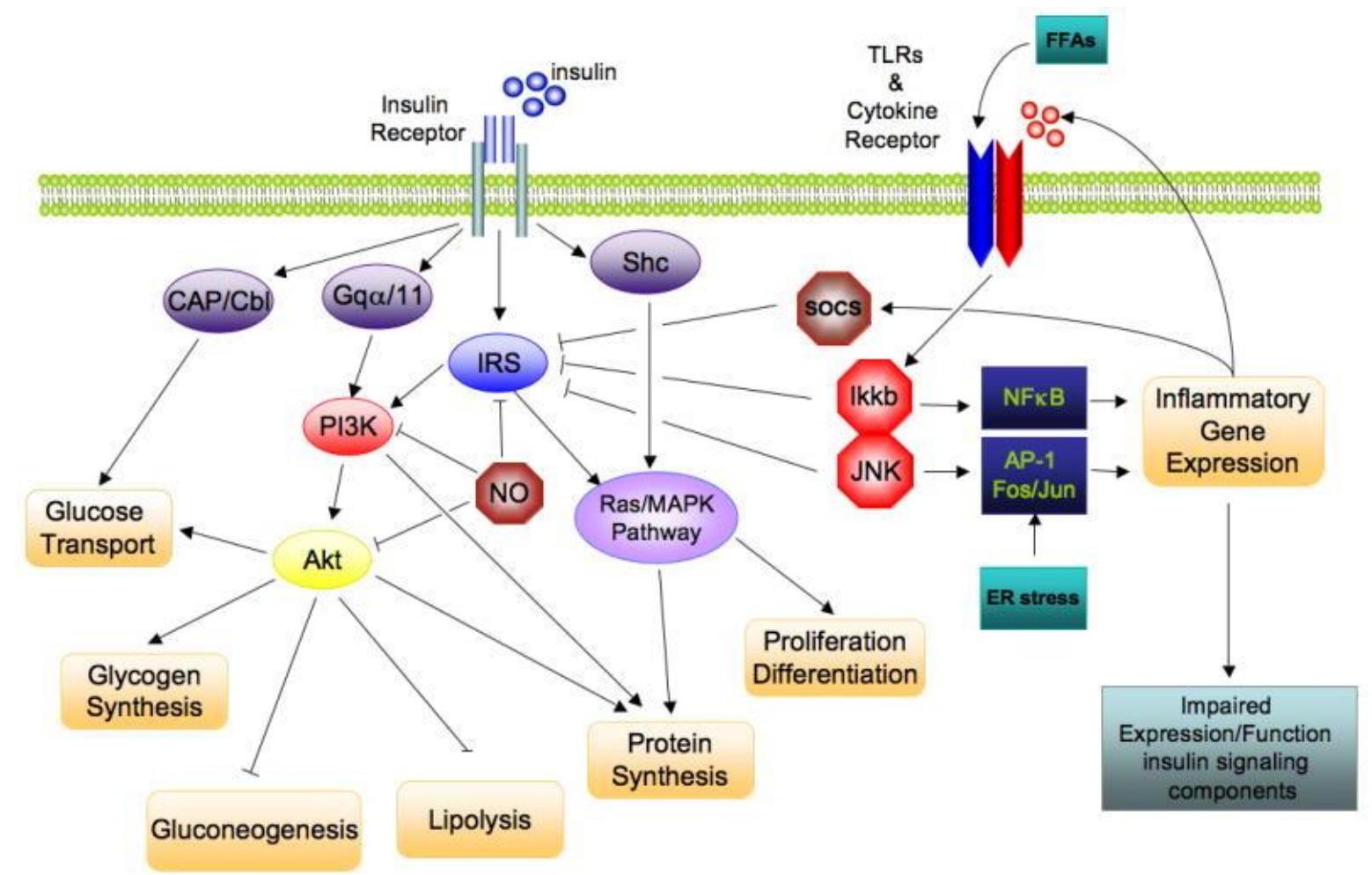

Figure 1.8 Inflammatory pathways implicated in insulin and FFA stimulation. Insulin binds cell surface insulin receptors to increase glucose uptake into cells predominantly through the phosphorylation of IRS, leading to the activation of the PI3K/AKT and Ras/MAPK signaling cascades to support growth and development. The abundance of FFA in the system hyperactivates TLRs and other chemokine receptors, causing an increase in phosphorylation of downstream inflammatory mediators like IKKB and JNK. Signaling through the NFKB and AP1 (Jun/Fos) pathway increases, leading to an increase but impaired expression of inflammatory components. The activation of IKKB and JNK serine kinases also inhibits the signaling ability of IRS, which further impairs conventional insulin signaling. In turn, other inflammatory signals like NO and SOCS also impairs the activity of IRS signaling, resulting in an overall increase in the production of inflammatory mediators and a decrease in normal glucose uptake and metabolism. Reprinted from FEBS Letters, 582/1, De Luca C et al, Inflammation and Insulin Resistance, Pages No. 97-105, Copyright 2008, with permission from Elsevier.

\subsubsection{ANGPTL4 and Inflammation}

The dysregulation of ANGPTL4 impacts lipid and glucose metabolism, leading to obesityinduced inflammation and the unwanted accumulation of fat and energy storage [221, 222]. The increase in FFA and SFA was found to positively regulate pro-inflammatory cytokine production and insulin resistance, the latter directly involved in mediating cellular glucose uptake [223, 224]. FFA and SFA also hyperactivates Toll-like receptor 4 (TLR4), resulting in the activation of an assortment of immune responses. In turn, this triggers the synthesis and secretion of increased levels of pro-inflammatory cytokines like TNF- $\alpha$ and IL-6 [225]. Augmented pro-inflammatory signals further activate downstream S6K/IRS/PI3K/AKT signalling cascade in a positive feedback manner, causing the eventual insulin resistance and development of type II diabetes. 
Numerous studies have implicated a role for ANGPTL4 in inflammation, where ANGPTL4 plays either anti- or pro-inflammatory roles in a context- or tissue-dependent manner. ANGPTL4 prevents foam cell formation by inhibiting fatty acid uptake into mesenteric lymph node macrophages, thus protecting against severe pro-inflammatory effects of saturated fatty acids [226]. ANGPTL4 also protects against atherosclerosis development [227] and acts as an angiogenic mediator in arthritis [227, 228]. ANGPTL4 was observed to exacerbate influenza and inflammation through independent IL-6-STAT3 and SIRT1-NFkB mediated pathways in the lungs [196, 229]. Interestingly, serum ANGPTL4 was correlated to $\mathrm{C}$ reactive protein (CRP) levels in type II diabetic patients, suggesting that ANGPTL4 might be involved in the inflammatory progression during the metabolic syndrome [230]. In the context of infection-associated inflammation, bacterial lipopolysaccharide (LPS) was found to be able to up-regulate ANGPTL4 expression in the mouse serum within 8 hours upon LPS stimulation and was subsequently labelled as an acute phase protein [231]. Furthermore, it was also shown that stimulating 3T3-L1 adipocytes with inflammatory chemokines like IL$1 \beta$, TNF- $\alpha$, IFN- $\gamma$ increases ANGPTL4 expression. Another independent group has also shown that the acute phase protein $\alpha 1$-antitrypsin (A1AT) increases ANGPTL4 expression through ERK1/2 signaling cascade [232]. Albeit the numerous reports of the role of ANGPTL4 in inflammation, the pathways by which ANGPTL4 modulates inflammation are largely unknown and remains unclear.

\subsubsection{ANGPTL4 and Cancer}

We consider the immune system as our friend; it protects us by fighting infections while keeping us healthy. But there is a darker side to the immune system. Often, when it comes to cancer, we find that the immune system can turn traitor and actually promote cancer development. The presence of leukocytes in tumors has been noted for many decades and provides the first clue that inflammation is linked to cancer. Yet it is only within the last few years that we have obtained clear evidence that inflammation plays a critical role in cancer development, and we are just beginning to understand the molecular mechanisms of how this happens. Indeed, chronic infections, obesity, smoking, alcohol consumption, environmental pollutants and high fat diets are now recognized as major risk factors for most common types of cancer; and, importantly, all these risk factors are linked to cancer through inflammation.

In an inflammatory and oxidative milieu, cells begin to accumulate damages to their DNA that facilitates the accumulation of pro-oncogenic mutations. Furthermore, the incessant secretion of ROS, MMPS, cytokines, chemokines and other soluble pro-inflammatory factors like CSF-1 and IL-6 by tumor-associated macrophages (TAMs) amongst other inflammatory infiltrates, potentiates angiogenesis and tumor progression [233]. TAMs also secrete TGF $\beta$, TNF- $\alpha$ and IL-10 [234, 235], and attenuating cytotoxic T cell activation [236]. Coupled onto sustained cell proliferation, reduced DNA repair and the subversion of apoptosis, cells in the chronic inflammatory environment develop neoplastic phenotypes and progress into cancer $[237,238]$. 
A role for ANGPTL4 in regulating inflammation has been implicated (see above, chapter 1.3.1.3). Conceivably, ANGPTL4 has a well-described role in carcinogenesis. ANGPTL4 is both a pro-angiogenic and anti-apoptotic factor that promotes endothelial vascularisation [209, 228, 239, 240]. Recently, we found that ANGPTL4 facilitates cell migration [215] and also regulates intracellular oxidative $\mathrm{O}_{2}^{-}: \mathrm{H}_{2} \mathrm{O}_{2}$ ratios in tumors [197], In addition, ANGPTL4 has also been implicated in cancer progression and metastasis. Independent groups have also reported that ANGPTL4 levels were increased in gastric cancer cells [241] and renal epithelial tumor cells [242, 243]. Nakayama et al and Shibata et al proposed that ANGPTL4 play significant roles in both venous and lymphovascular invasion [241, 244], suggesting that ANGPTL4 affects tumor migration and metastasis. Others identified ANGPTL4 as a marker of breast-lung metastasis [207] and oral squamous cell carcinoma [245]. These findings suggest that ANGPTL4 is a potential regulator in cancer progression and tumor vascularisation. Even though ANGPTL4 has been identified to play vital roles in regulating both inflammation and tumorigenesis, the exact roles by which ANGPTL4 regulates cancer through inflammatory pathways remain to be elucidated. Therefore, it is of utmost importance to understand the relationship between the ANGPTL4-dependent, inflammationtumorigenesis signalling axis. 
2 AIMS 
We aim to explore various ANGPTL4-dependent intermediates that regulate the inflammatory landscape. Using independent models, we further delineate the respective signaling cascades that primarily govern both the acute and chronic phases of inflammation in the inflammation-cancer signaling axis.

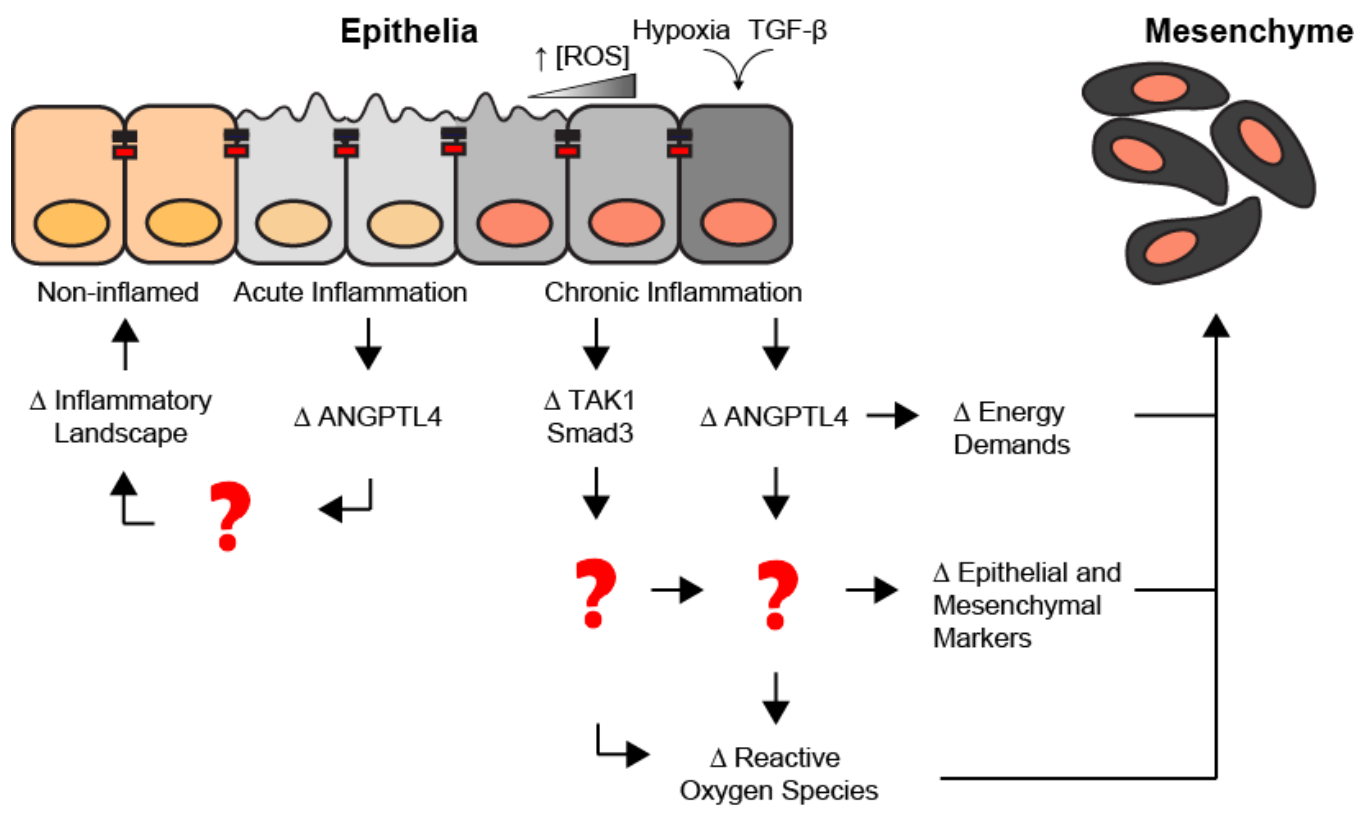

Figure 2.1 A graphical summary of ANGPTL4-dependent signaling cascades during acute and chronic inflammation which promotes epithelial-to-mesenchymal transition.

In PART I of this thesis, we demonstrate that the importance of ANGPTL4 in regulating the acute colonic inflammatory environment.

I. An anti-inflammatory role for colonic ANGPTL4 in dextran sulfate sodium salt (DSS)-induced colitis and dietary stearic acid (SA) intake.

II. The role of the commensal gastrointestinal microbiota in inflammation.

III. The intrinsic role of colonic ANGPTL4 in regulating leukocyte infiltration during DSS-induced inflammation, and thus, the colonic inflammatory landscape.

IV. The underlying mechansims involving the regulation of Tristetraprolin (TTP) through CREB and NF- $\kappa B$ transcription factors.

In PARTS II and III of this thesis, we aim to understand the role of TAK1 and ANGPTL4 in regulating ROS production during the chronic inflammatory state in the tumor microenvironment.

V. The role of TAK1 and ANGPTL4 in mediating the pro-inflammatory ROS landscape in the early, pre-tumor microenvironment.

VI. To understand the role of ROS in promoting field cancerization.

VII. To delineate possible signalling downstream of ANGPTL4 during chronic inflammation that promotes tumor cell migration and the epithelial-mesenchymal transition (EMT) process. 
3 METHODOLOGY 


\subsection{METHODOLOGICAL HIGHLIGHTS}

Detailed experimental methodologies can be obtained in the individual papers and manuscripts. Therefore, this section will describe methodological highlights utilized in this thesis.

\subsubsection{Bone Marrow Tranplantation}

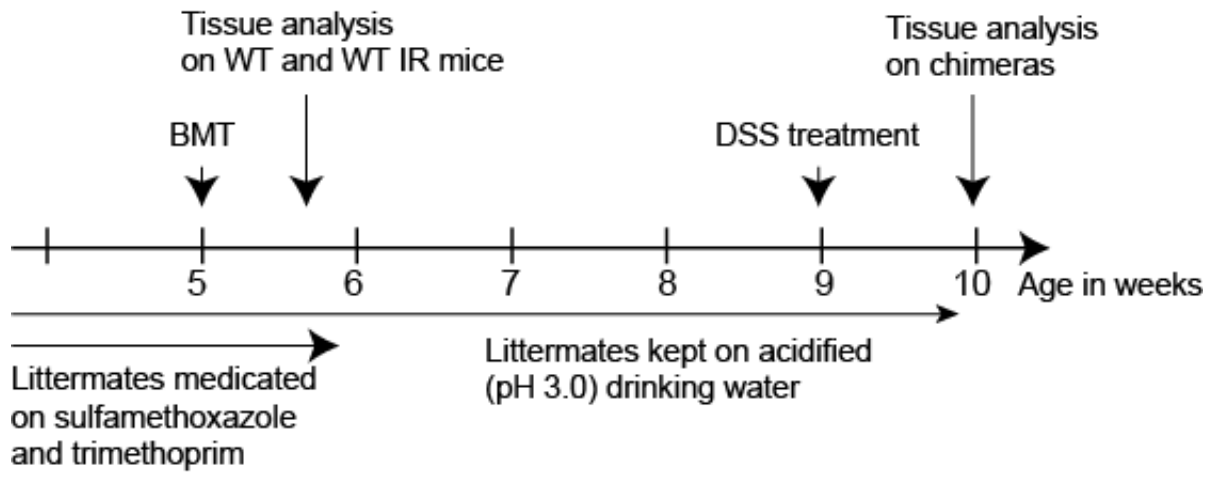

Figure 3.1 Experimental Timeline for the Bone Marrow Transplant.

To exclude the cell-autonomous (intrinsic) effects of ANGPTL4 originating from non-colonic epithelial cells, we further performed a bone marrow transplant using the bone marrow from donor ANGPTL4 $^{-/-}$or ANGPTL4 ${ }^{+/+}$mice to recipient ANGPTL4 $^{+/+}$mice (Figure. 3.1). Recipient mice were kept on acidified water $(\mathrm{pH} 3.0)$ over the course of the entire experiment. BMT (ANGPTL4 ${ }^{-/}$) and BMT $\left(\right.$ANGPTL4 $^{++}$) chimeras were medicated with trimethoprim $(8 \mathrm{mg} / \mathrm{kg}$ ) and sulfamethoxazole $(40 \mathrm{mg} / \mathrm{kg})$ in oral suspension (Allpets Asia, Singapore) for one week before and after lethal $\gamma$-irradiation (9.5 Gy), using the Biobeam 8000 (Gamma-Service Medical GmbH).

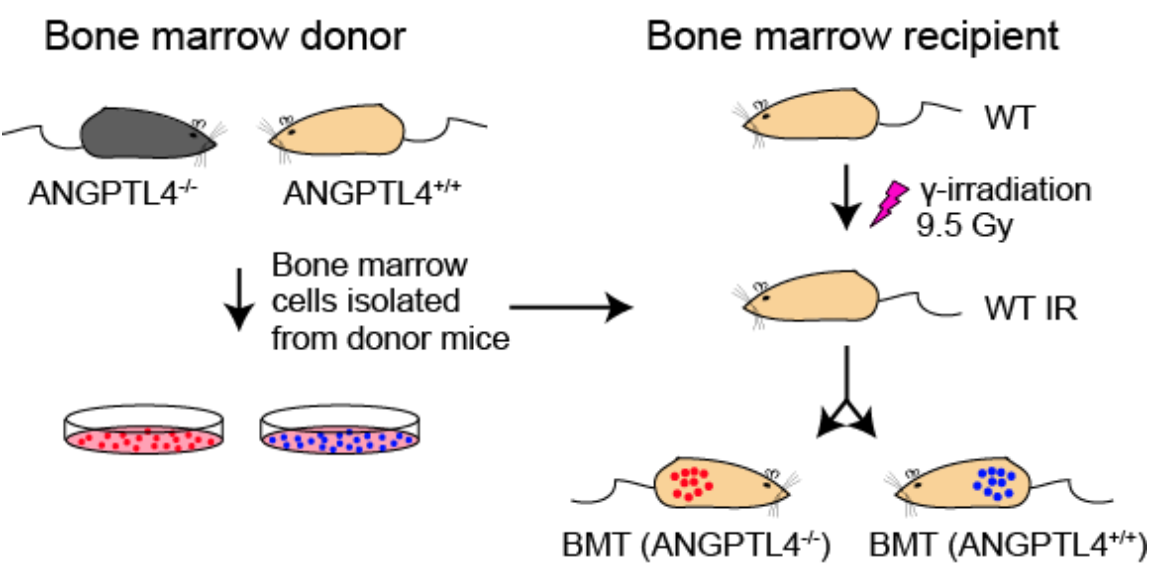

Figure 3.2 Bone Marrow Transplant Procedures.

Donor bone marrow cells from the humerus, tibia and femur of donor mice were isolated in serum-free DMEM. Cells were first washed with PBS followed by red blood cells lysis $\left[0.89 \% \mathrm{NH}_{4} \mathrm{Cl}(\mathrm{w} / \mathrm{v}), 0.1 \mathrm{mM}\right.$ EDTA, $\mathrm{pH}$ 7.2]. Cells were filtered through a $30 \mu \mathrm{m}$ nylon cell 
strainer before depleting mature $\mathrm{CD}^{+}$and $\mathrm{CD}^{+}$donor immune cell populations (QuadroMACS kit with anti-CD4- and anti-CD8a-conjugated microbeads, \#130-049-201, \#130-049-401 and \#130-091-051; from Miltenyi Biotec; in accordance to manufacturer's recommendations; degassed, ice cold PBS containing 0.5\% BSA, 2 mM EDTA at pH 7.2). Cells were resuspended in serum-free DMEM and approximately $10^{7}$ bone marrow cells (in $100 \mu \mathrm{L}$ total injected volume) were introduced into WT IR recipient mice via retro-orbital injection (Figure 3.2). BMT (ANGPTL4 ${ }^{-/}$) and BMT $\left(\mathrm{ANGPTL}^{+/+}\right.$) chimeras were allowed to recover for 4 weeks before the subsequent 8 -day $2 \%$ DSS treatment.

Hematopoietic reconstitution was evaluated using genotype PCR. At experimental endpoint, cell counts were performed for the whole femur and spleen tissues using the ADAM-MC cell counter (NanoEntek, USA). Splenic and colonic cells were analyzed for F4/80 and CD11b expression using FACS (BD LSRFortessa X-20) to determine the extent of immune cell infiltration.

\subsubsection{S Metagenomics Sequencing}

Age-matched, post-weaned ANGPTL4 ${ }^{-/-}$and ANGPTL4 ${ }^{++}$males were co-housed for the entire experiment. Fresh fecal samples were collected from both ANGPTL4 ${ }^{-/}$and $\mathrm{ANGPTL}^{+/+}$littermates before and after 8-day 5\% DSS treatment, and stored at $-80{ }^{\circ} \mathrm{C}$. Bacterial genomic DNA was isolated using the FASTDNA spin kit for feces in accordance to manufacturer's recommendations (MP Biomedicals, USA; \#116570200). 16S metagenomics sequencing was performed by SeqMatic, USA, using primers targeting the 16S V4 region by means of the high throughput NGS Illumina MiSeq platform. 


\subsubsection{Transwell Migration Assay}

THP1 monocytes were initially differentiated into macrophages by incubating cells in DMEM supplemented with 10\% FBS and $100 \mathrm{ng} / \mathrm{mL}$ TPA for $48 \mathrm{~h}$. Cells are left to recover for $24 \mathrm{~h}$ before seeding onto transwell inserts. Concurrently, respective siRNA knockdowns were performed on iCECs (iCEC $\mathrm{Ctrr}_{\text {, }}$ iCEC ANGPTL4 $_{4}$ and iCEC $_{\text {TTP }}$ ) that were pre-seeded onto wells. Cells on both the inserts and wells are kept apart until the start of the transwell migration experiment as shown in Figure 3.3.

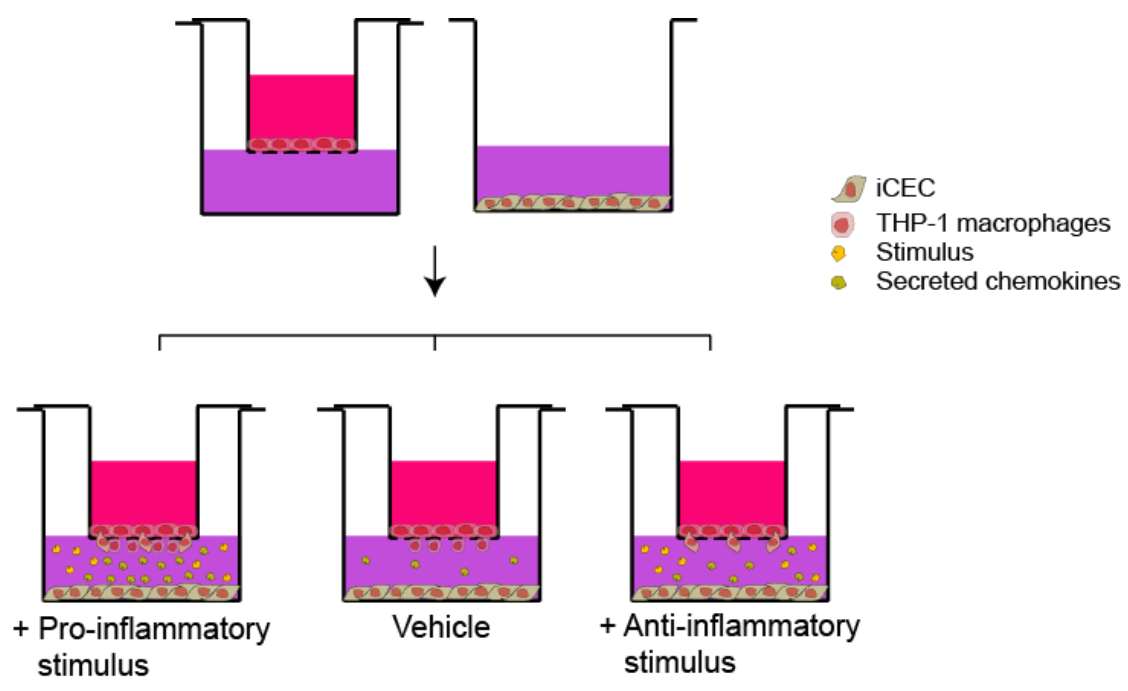

Figure 3.3 A graphical illustration of the transwell migration assay set up.

Inserts containing differentiated THPI-derived monocytes were then introduced to the respective transfected iCECs and exposed to various pro- or anti-inflammatory stimuli for 10 h. Subsequently, inserts were then removed and rinsed twice with PBS and fixed in $1 \%$ glutaraldehyde for $10 \mathrm{~min}$. Inserts were rinsed again and stained with SYTO 60 (Thermo Fisher, USA) for $30 \mathrm{~min}$. Consequently, cotton buds were used to scrape away all nonmigrated THP1 cells trapped within the upper chamber of each insert and rinsed for the last time in PBS. Relative fluorescence was quantified using the CLx scanner and Image Studio V2.1 (LI-COR Biosciences, USA).

\subsubsection{Statistical Analysis}

Statistical analyses were performed using 2-tailed Mann Whitney U-test using Graphpad Prism 5 and Microsoft Excel software. P values are expressed as means \pm standard error; and $\mathrm{p}<0.05$ represent statistically significant differences, where $*$ denotes $\mathrm{p}<0.05 ; * * \mathrm{p}<0.01$ and $* * * \mathrm{p}<0.001$. 
4 RESULTS AND DISCUSSION 


\subsection{ANGIOPOIETIN-LIKE 4 IN ACUTE INFLAMMATION}

Recent studies report that Angiopoietin-like 4 (ANGPTL4) plays a multi-faceted role in the regulation of host inflammatory response. ANGPTL4 has been observed to play angiogenic roles in mediating arthritis [227, 228] and atherosclerosis development [227]. ANGPTL4 was found to exhibit anti-inflammatory functions in preventing macrophages from absorbing fatty acids, hence preventing foam cell formation [226]. However, others attribute ANGPTL4 to exacerbating inflammation. Serum C-reactive protein (CRP) levels have been correlated to ANGPTL4 expression in patients with type II diabetes [230]. LPS was detailed to positively up-regulate ANGPTL4 expression [231]. To date, ANGPTL4 has been shown to exert both anti- and pro- inflammatory responses through a wide array of stimuli. However, little is known about how ANGPTL4 regulates inflammation.

In Part I of this thesis, we sought to outline possible mechanisms that could explain the antiinflammatory role of ANGPTL4 (Figure 4.1). Using both dietary C18 saturated fatty acid (stearic acid; SA) and dextran sodium salt (DSS) as separate models to induce acute inflammation in mice, we show that mice exhibited an exacerbated inflammatory response in the absence of ANGPTL4. Confirming the current paradigm, 16S metagenomics sequencing showed little differences in the microbiota communities between the genotypes at steady state but show divergence during DSS-induced inflammation [246-248]. Both microarray analysis and bone marrow transplantation experiments further affirm the importance of epithelialderived ANGPTL4 in regulating the local inflammatory landscape. Subsequently, we demonstrated an increased stability in a subset of chemokines that were previously found to be regulated by an mRNA destabilizing protein, TTP. To reconfirm our in vivo findings, we subjected SV40-immortalized human colonic epithelial cells (iCECs) to pro- and antiinflammatory treatments. We observe that ANGPTL4 and TTP expression decreases with pro-inflammatory treatments and increases with anti-inflammatory stimulation. In summary Paper I demonstrated that ANGPTL4 is able to regulate the inflammatory landscape through TTP-dependent and TTP-independent pathways. 


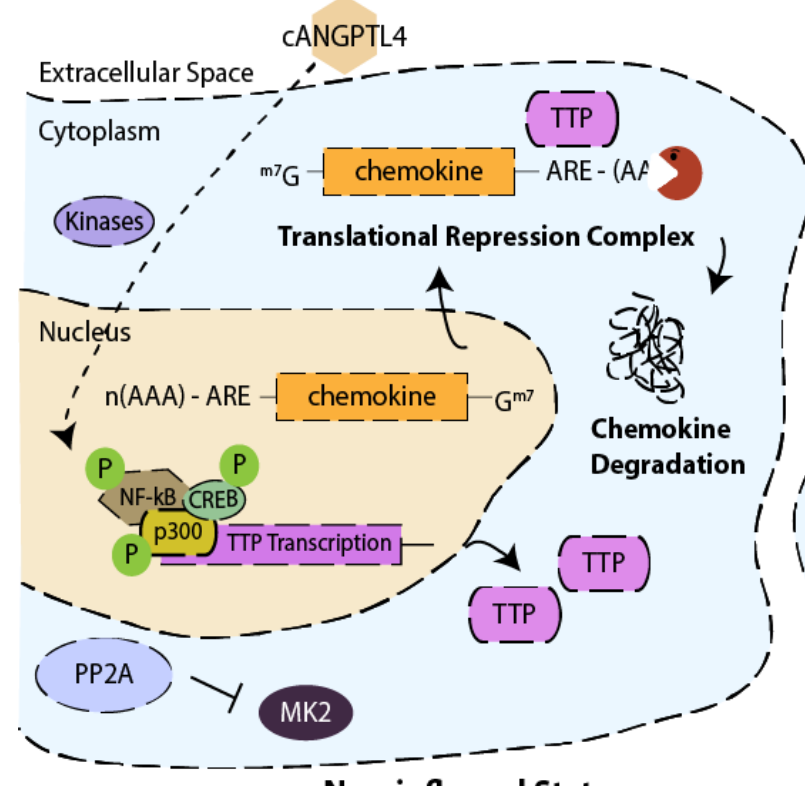

Non-inflamed State

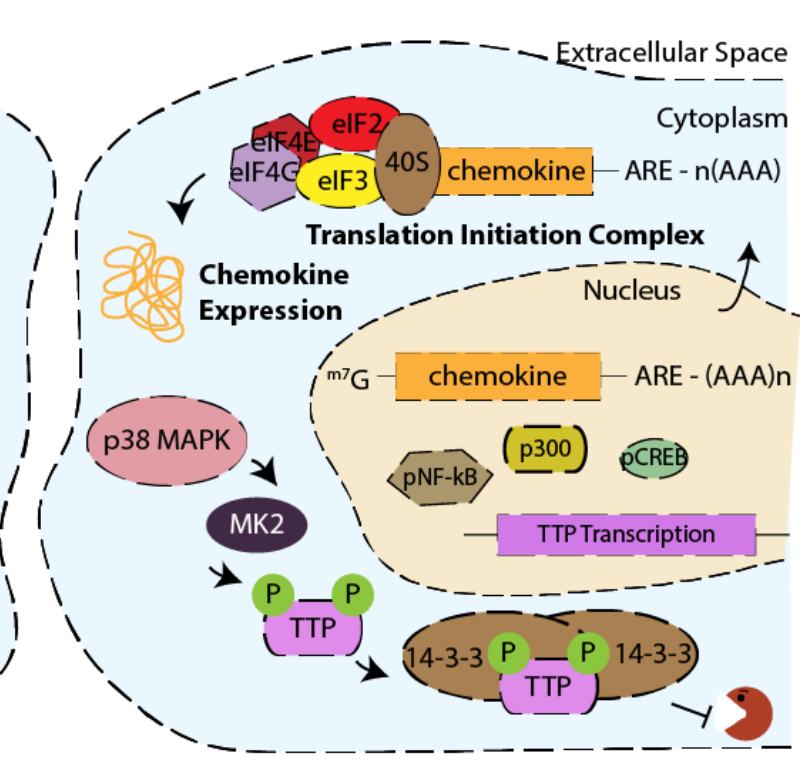

Inflamed State

Figure 4.1 A graphical summary of the TTP-dependent ANGPTL4 signaling axis. CANGPTL4 mediates colon epithelial cell homeostasis by regulating TTP expression. At basal state, CANGPTL4 stimulation maintains TTP expression through the phosphorylation of transcription factors CREB and NF-KB. TTP binds at the AU-rich regions of the chemokine 3' UTR, causing the degradation of target chemokine mRNA transcripts. During inflammation, positive p38MAPK-MK2 signaling causes the phosphorylation of TTP, allowing the 14-3-3 complex to sequester and inactivate TTP. Chemokine transcription ensues, bringing about increased amounts of chemotactic signals to aid the infiltration of immune cells and to remove the source of inflammation.

In a similar genome-wide study aimed at identifying the differences in the gene expression between UC and Crohn's disease (CD), Wu et al found that even though UC and CD resulted to similar clinical IBD characteristics, each disease exhibited a unique and non-overlapping pattern of genomic expression (GSE6731) [249]. Using their study cohort of CD, we conducted a comparative microarray gene expression analysis of $\mathrm{CD}$ patients against our murine ANGPTL4 ${ }^{+/+}$and ANGPTL4 ${ }^{-/-}$colon samples (Figure 4.2).

Unlike UC, the gene expression and hierarchical clustering profile was very different between human $\mathrm{CD}$ and murine samples (Figure 4.2a). Gene ontology analysis demonstrates that top molecular and cellular functions include cellular movement, cellular growth and proliferation, as well as cellular function and maintenance (Figure 4.2b). Further inquisition identified a region of differentially expressed inflammatory genes (black box demarcated in Figure 4.2a), but primary canonical pathways that were affected were not confined to the gastrointestinal tract (Figure 4.2c). One conceivable explanation to this difference is the varying aetiology and manifestation of $\mathrm{UC}$ and $\mathrm{CD}$ even though they belonged to the category of IBD. UC is limited to the colon, while $\mathrm{CD}$ has been reported to mostly affect the ileum and the colon, as well as other regions of the gastrointestinal tract. Recent correlation studies also suggest that CD might also be hereditary, and that genetic factors (mutations in genes like NOD2, IRGM, ATG16L1) are also believed to be contributing to the development of CD [250, 251]. 
a
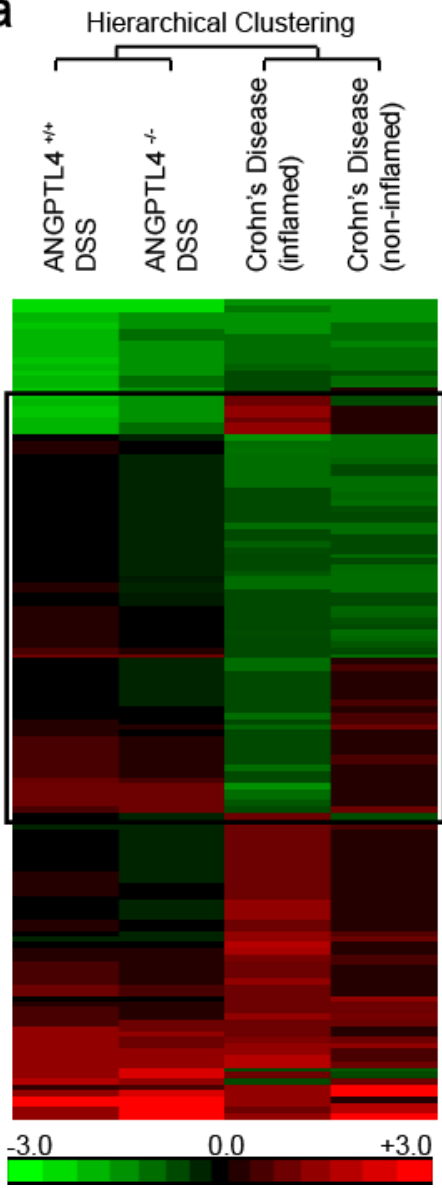

b

Molecular and Cellular Functions

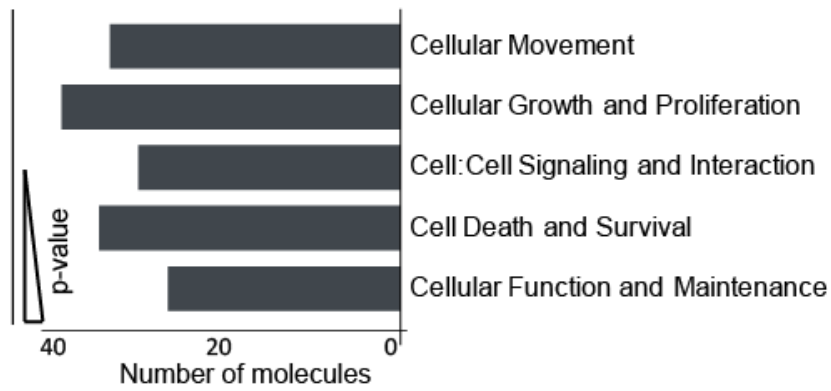

C

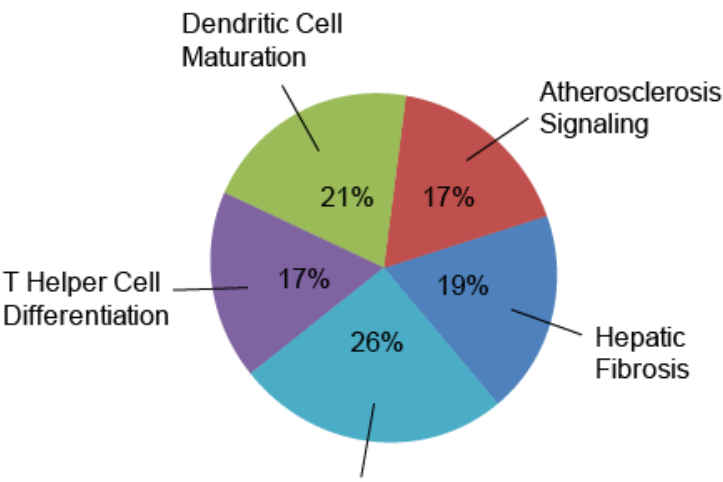

Role of macrophages,

fibroblast and

endothelial cells in

rheumatoid arthritis

Figure 4.2 Comparative microarray gene expression analysis between colonic samples from DSSchallenged mice and human Crohn's disease. (a) Microarray heat map showing changes in gene expression between murine ANGPTL4 ${ }^{+/+}$and ANGPTL4 ${ }^{-/-}$mice treated with DSS, and inflamed and non-inflamed Crohn's disease (CD) biopsies (GSE6731). (b) The IPA database ranks genes responsible for regulating cellular movement, cellular growth and proliferation, as well as cellular maintenance amongst the most significantly varied between murine and human samples. (c) Inflammatory genes that were found to be differentially expressed (as demarcated by the black box in figure 4.2a), were primarily responsible for the regulation of inflammatory processes not limited to the gastrointestinal tract. 


\subsection{TAK1 DEFICIENCY PROMOTES PRO-INFLAMMATORY ROS LANDSCAPE FOR FIELD CANCERIZATION}

As a classical theory that connects inflammation and cancer, the sustained state of chronic inflammation and irritation has frequently been correlated to drive spontaneous tumor development [238]. Epithelial cells in the chronic inflammatory environment are exposed to ROS and are at risk of accumulating unwanted DNA mutations that could further impede tumor suppressor mechanisms in place to prevent tumor formation. Under typical circumstances, the accumulation of ROS-dependent DNA damage or genomic instability would result in senescence or apoptosis via the p53 pathway [252]. However, most tumor cells are incapable of responding to the accumulation of oxidative stress levels because of defective anti-oxidant signaling pathways [253], which further leads to tumor malignancy.

In Part II of this thesis, we capitalized on a variety of tumorigenic cultures like A5RT3, MKN78, HSC-5 and the non-metastatic Hacat to show that epithelial to mesenchymal transition (EMT) is favored with TGF $\beta$ stimulation through the canonical Smad3 signaling axis. Interestingly, Paper II showed that the depletion of TAK1, a non-canonical TGF $\beta$ signaling partner, further promotes cytoskeleton remodeling and EMT. Similar to other groups, we validate a sustained production of ROS in TAK1-ablated cells [254, 255]. We further demonstrate an increase in Rac1-Nox1 activation and a corresponding decrease in RhoA activity in TAK1-depleted cells, which further elevates ROS levels in the microenvironment. An increase in Rac1-Integrin $\beta 1$ interaction and an augmented expression of mesenchymal markers like vimentin, N-cadherin, fibronectin and Snai1/2 also hint at the initiation of cell migration. An increase in cell traction force was also recorded for cells depleted of TAK1. Put together, our data suggests a regulatory role of endogenousTAK1 in Rac-RhoA activation in limiting ROS production, as well as cytoskeleton remodeling and cell migration (Figure 4.4).

Follow-up investigations in a related publication reveal that $\mathrm{H}_{2} \mathrm{O}_{2}$ levels are positively correlated to increasing tumor aggressiveness and the intensity of diffused $\mathrm{H}_{2} \mathrm{O}_{2}$ throughout the tumor epithelial and stromal regions is amplified with increased malignancy [256]. Since the early days of cancer treatments, surgeons and oncologists alike have reported a rising trend of patients developing recurrent second primary malignancies even after the successful removal of the index tumors. As such, Slaughter et al first coined the term 'field cancerization' in 1953 when they first demonstrated histological changes in the epithelium that resided beyond the tumor boundary [257]. The heterogeneous population of cancerassociated fibroblasts (CAFs) which reside in the adjacent stroma is also believed to sustain tumor proliferation and metastasis. However, due to the complexity of defining the region beyond the tumor that possesses malignant transformations, research contributions towards field cancerization remains in its infancy. To date, it was noted that almost $30 \%$ of all cancerrelated deaths are attributed to second primary malignancies and this remains an important finding in cancer treatment regimens and research. 


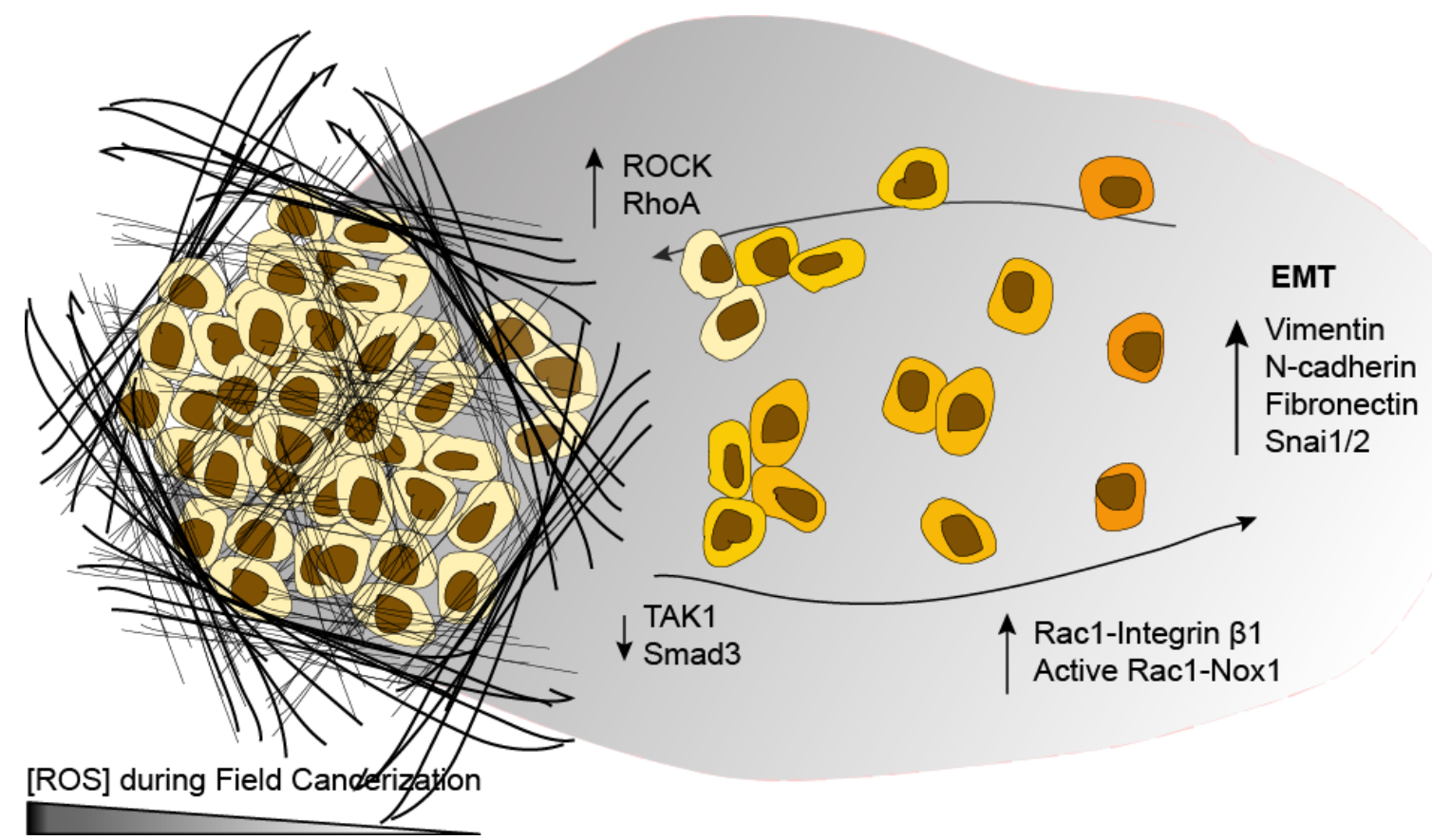

Figure 4.4 A graphical summary of the TAK1-dependent signaling cascade which promotes epithelial-to-mesenchymal transition. TAK1 activity in benign cells protects against the TGF $\beta$-Smad3 stimulation in the tumor environment. As cells lose TAK1 expression, cells respond by increasing Rac1-Integrin $\beta 1 / 3$ interactions, promoting increased activity of Rac1-Nox1 complex formation. This association increases the production of reactive oxygen species (ROS), which further fuels the upregulation of inflammatory cytokines, resulting in the formation of a chronic inflammatory landscape in the tumor microenvironment. Elevated ROS levels also suppress the expression of epithelial marker E-cadherin but raise the levels of mesenchymal markers like vimentin and $\mathrm{N}$-cadherin. In this respect, undergo cytoskeleton remodeling, allowing for the increase the number of focal adhesions and stress fibers to facilitate migration.

Experimental findings reveal that not only does $\mathrm{H}_{2} \mathrm{O}_{2}$ increase the invasiveness of epithelial cells, $\mathrm{H}_{2} \mathrm{O}_{2}$ also disrupts cellular homeostasis by oxidizing key signaling intermediates like PTEN, Src, JNK and EGFR. We show that fibroblasts incubated with the conditioned media of CAFs experience a similar oxidative stress with those treated with exogenous $\mathrm{H}_{2} \mathrm{O}_{2}$. Along with the reduction of Smad3 and TAK1 expression, our data show that $\mathrm{H}_{2} \mathrm{O}_{2}$-treated fibroblasts quickly become desensitized to TGF $\beta$ stimulation. Similar to our earlier findings, we demonstrate that the reduction in Smad3 and TAK1 signaling further increases ROS production and $\mathrm{p} 65-\mathrm{NF}-\kappa \mathrm{B}$ activity in fibroblasts, elevating the overall oxidative stress while promoting a pro- inflammatory microenvironment. These combined conditions not only cause cells to develop resistance to TGF $\beta$ signaling, it also primes the conversion of fibroblasts to become CAFs. Interestingly, we found that CAFs express low levels of Gpx1, rendering them incapable of detoxifying ROS and hence, allowing for the accumulation of oxidative stress. In all, our data is in agreement that ROS-induced chronic inflammation is sufficient to give a pro-inflammatory and pro-tumor microenvironment which promotes field cancerization in the epithelium. 


\subsection{ANGPTL4 PROMOTES TUMOR CELL MIGRATION AND EMT}

In complex diseases like cancer, both the host immune system and the tumor (tumor cells and cells that make up the tumor microenvironment) play crucial roles in maintaining the dynamics that keep the cancer viable.

Huang et al show that tumor cells express high levels of ANGPTL4 and that cANGPTL4 interacts with junction proteins like claudin-5, VE-cadherin and integrin $\alpha 5 \beta 1$ to disrupt endothelial continuity and vascular integrity [198]. Kubo et al also reported that cancer cells exposed to hypoxic environments increased ANGPTL4 expression [258] while Tanaka et al found that ANGPTL4 increases the metastatic potential of tumorigenic cells [245]. Zhu et al demonstrated that ANGPTL4 levels correlated to increase ERK-AKT signaling in cancer, as well as increased E-cadherin and decreased vimentin expression [259], a feature that is characteristic of the EMT process.

To understand the medical relevance of ANGPTL4, we first characterized the expression of cANGPTL4 in human tumor biopsies. We observed that cANGPTL4 is elevated with increasing stages of cancer in a stage-dependent manner (Figure 4.5a). In a submitted manuscript (Paper III), we also demonstrated that ANGPTL4 drives the EMT process by elevating cellular energy flux through 14-3-3 $\gamma$-dependent signaling cascades. We also found that ANGPTL4 phospho-activates AKT and AMPK in cancer cells during EMT and might be a possible modulator of cellular bioenergetics. Using polarized gastric carcinomas MKN74 cells, we show that the induction of EMT accompanied by an increase in Glut 1 expression occurs within 48 hours post hypoxia exposure. Interestingly, this same hypoxia exposure delayed EMT in cells with impaired ANGPTL4 signaling (treated with neutralizing cANGPTL4 antibody; $\alpha$-cANGPTL4). However, stimulation with recombinant cANGPTL4 in normoxic conditions caused cells to lose their epithelial morphology and E-cadherin expression within 48 hours and was sufficient to drive cells into EMT (Figure 4.5b-c). 
a

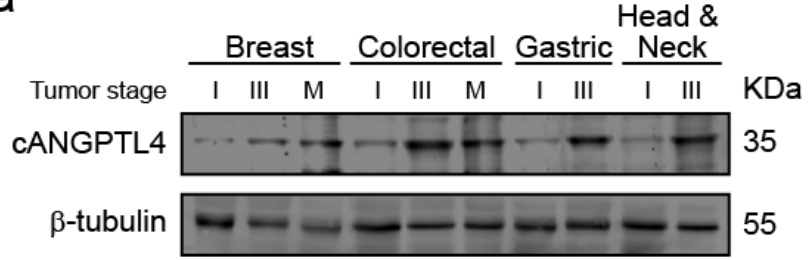

b

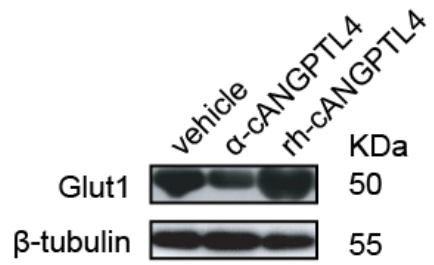

Normoxia + rh-cANGPTL4

C

\begin{tabular}{ll} 
Hypoxia & \\
\hline &
\end{tabular}

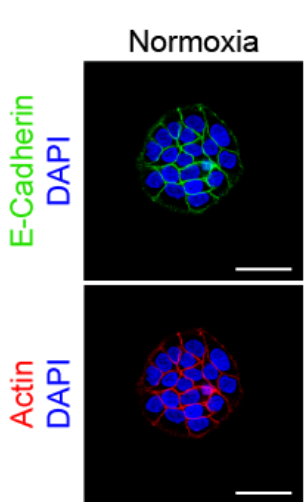

d

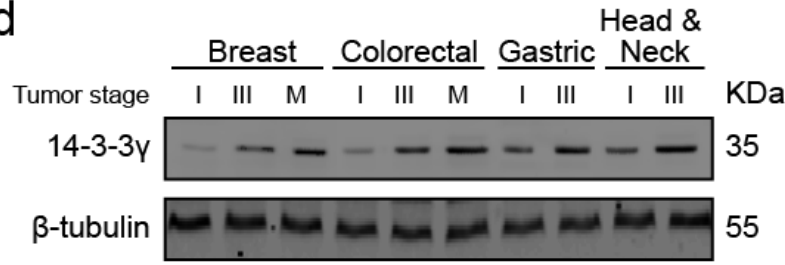

e

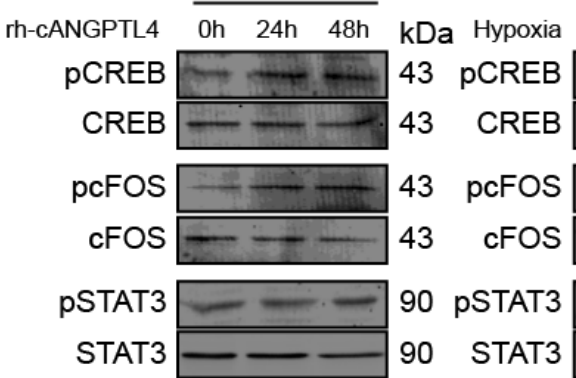

STAT3
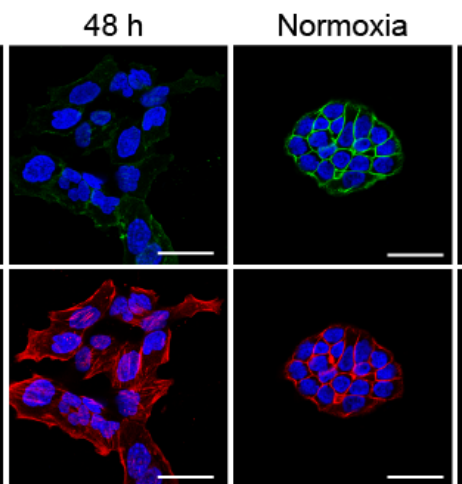

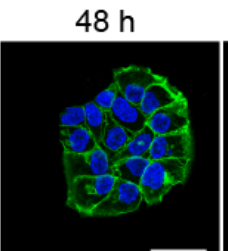

Vehicle $48 \mathrm{~h}$

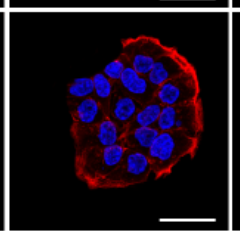

f

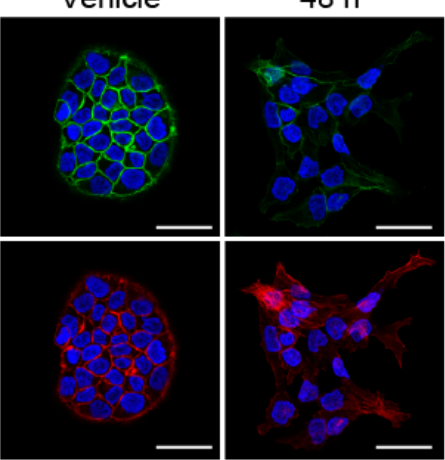

\begin{tabular}{|c|c|c|c|c|}
\hline IP: TSC2 & \\
\hline Hypoxia & $\begin{array}{lll}\text { Oh } & 24 \mathrm{~h} & 48 \mathrm{r} \\
\end{array}$ & Oh & $24 \mathrm{~h}$ & $48 \mathrm{~h}$ \\
\hline IB:TSC2 & -5 & $\overline{-}$ & - & - \\
\hline $\begin{array}{r}\text { B:p-TSC2 } \\
(\mathrm{S}-939)\end{array}$ & $\cdots$ & $=$ & & $\cdots$ \\
\hline$B: 14-3-3$ & 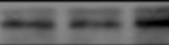 & - & 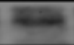 & $=x+x+3$ \\
\hline
\end{tabular}

Figure 4.5 ANGPTL4 promotes cytoskeletal remodeling during epithelial to mesenchymal transition (EMT) and alters cancer cell bioenergetics. (a-b, d) Representative immunoblot analysis of (a) CANGPTL4 and (d) 14-3-3 $\gamma$ in human samples (purchased from Proteogenex, USA), of breast, colorectal, gastric and head and neck cancer samples with varying tumor stages and (b) Glut 1 in $\alpha$ cANGPTL4- and rh-cANGPTL4-treated MKN74 cells. (c) Immunofluorescence staining of the epithelial marker E-cadherin (in green) and phalloidin for the actin cytoskeleton (in red) in MKN74 gastric carcinoma cells, counterstained with DAPI (in blue). MKN74 cells were treated with rh-cANGPTL4 in normoxia (right panel), in hypoxia (middle panel) and in hypoxia with human cANGPTL4 antibody ( $\alpha$ CANGPTL4; left panel) for 48 hours. A sharp decrease in E-cadherin expression and a remodeling of the actin cytoskeleton was observed at 48 hours with both rh-cANGPTL4 and hypoxia treatment. On the other hand, treatment with $\alpha$-CANGPTL4 antibody rescued this loss and promoted EMT induction, suggesting that ANGPTL4 plays crucial roles in cytoskeletal remodeling during EMT. (e) Representative immunoblot analysis showing CREB/pCREB, cFOS/pcFOS and STAT3/pSTAT3 expression in rh-CANGPTL4- and hypoxia-stimulated MKN74. Immunoblot analysis showing the expression of indicated proteins after co-immunoprecipitation of (f) TSC2, (g) 14-3-3 $\gamma$ or Snai1 during hypoxia-induced EMT in MKN74 ctrl $_{1}$ and MKN74 ${ }_{\text {ANGPTL4 }}$ cells. 
14-3-3 belongs to a family of highly conserved adaptor proteins and has been previously reported in regulating cell cycle proteins Chk1 [260], ATM and CDC25 [261], and also acute phase TTP during inflammation (Paper 1). In addition, we previously found that ANGPTL4 augmented 14-3-3 expression during wound healing [195, 215], suggesting that ANGPTL4 could modulate 14-3-3 during EMT. In addition to EMT-induced MKN74, we found that tumors at higher stages express elevated levels of 14-3-3 $\gamma$ but not other 14-3-3 isotypes (Figure 4.5d). Using a combination of the unbiased kinase inhibitor arrays and in silico analysis using the IPA software, we report that ANGPTL4 regulates 14-3-3 $\gamma$ transcription through transcription factors like CREB, cFOS and STAT3 (Figure 4.5e). The ANnotation and Integrated Analysis of the 14-3-3 interactome (ANIA) database also identified TSC2 and Snail as likely interaction partners of the 14-3-3 complex. To strengthen our findings, proximity ligation assays and co-immunoprecipitation experiments reveal an increase in the

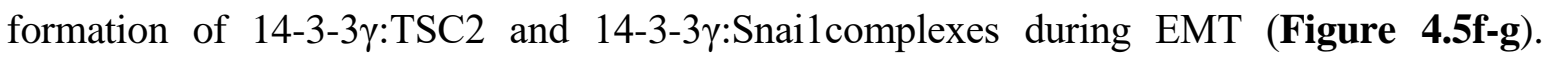
Similarly, we also observed significantly more 14-3-3 $\gamma$ :TSC2 and 14-3-3 $\gamma$ :Snailcomplexes in aggressive human tumors biopsies as compared to their benign controls. We also demonstrate that 14-3-3 $\gamma$ stabilizes Snail binding to the E-cadherin promoter region during EMT, allowing for the transcription of the E-cadherin. Put together, our findings show that ANGPTL4 exert control over the EMT process by stabilizing key EMT proteins and meeting the increased cellular energy demands through AKT-dependent pathways. More importantly, our data underscores the significance of the ANGPTL4: 14-3-3 $\gamma$ signaling axis during EMT.

Interestingly, Wang et al, Griseri et al and Young et al recently discussed about the importance of proteins like TTP and HuR that can regulate cytokine stability play important roles in inflammation-related cancers and also in cancer progression [262-264]. In this respect, the dysregulation in cytokine stability after the initial inflammatory phase allows for a persistent feedback loop of increased inflammatory signals (chronic inflammation) and generation of ROS around the developing tumor. Since there is a need for increased nutrient supply to support the growing tumor, genes that promote angiogenesis should also be highly expressed in the vicinity.

To date, independent groups have brought forth convincing evidence to suggest that various cancers over-express immune checkpoint proteins as a method to evade immuno-surveillance [265]. Evolutionarily, the binding of PD-1 from B and T cells to its ligands PD-L1/2 negatively regulates $\mathrm{T}$ cell activation, muting out immune responses to prevent protracted immune reactions and tissue injury. It was found that immune cells in the pro-inflammatory tumor microenvironment do not mount any anti-cancer response because of the increased PD-1/PD-L1 signaling, further lowering $\mathrm{CD}^{+} \mathrm{T}$ cell survival, proliferation and cytokine production [266, 267]. 
5 CONCLUDING PERSPECTIVES 
The advancement of Science and Research is both rapid and brisk. In a short span of time, seemingly new findings can pave the way to become novel applications aimed at increasing the efficiency of diagnostics. Hence, the need to keep abreast of new findings is crucial to pilot new experiments in directions that pioneer novel work. In this thesis, we explored possible regulatory pathways of ANGPTL4 in both acute and chronic inflammation, and also examined its role in promoting EMT and tumor metastasis.

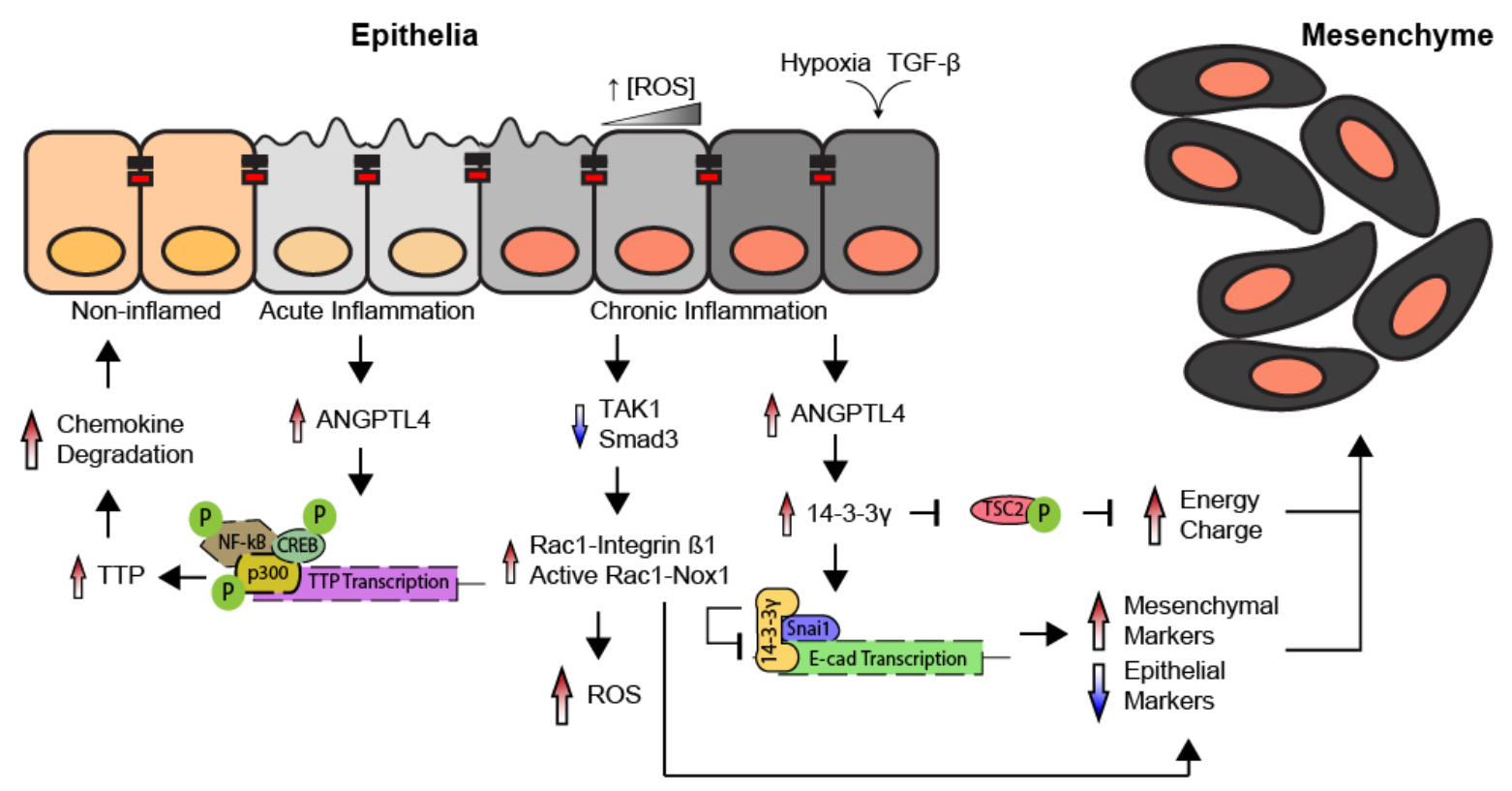

Figure 5.1 A graphical illustration showing ANGPTL4-dependent regulatory pathways during both acute and chronic inflammation. The induction of ANGPTL4 expression during acute inflammation initiates immune cell infiltration to the site of injury. Subsequently, ANGPTL4 stimulates TTP transcription, a RNA-binding intermediate which destabilizes cytokines. This quenches the local proinflammatory landscape and returns the tissue to its non-inflamed state. During chronic inflammation, a tightly regulated shift in ANGPTL4 and TAK1/Smad3 signaling disrupts cellular homeostasis. Cells desensitize towards the steady state of inflammation and accumulate increasing amounts of reactive oxygen species. The increase in expression of mesenchymal:epithelial markers promotes cytoskeleton remodeling and causes cells to adopt a more mesenchymal phenotype. Augmented $14-3-3 \gamma$ also inhibits TSC2, accommodating for the increase in cellular energy demands during EMT.

We began by exploring the value of ANGPTL4 in regulating the immune landscape in both acute and chronic inflammatory states, leading up to cancer formation, EMT and metastasis (Figure 5.1). For the reason that functional ANGPTL4 exists in two forms with nonoverlapping roles (dimeric or tetrameric nANGPTL4 is primarily involved in energy metabolism while monomeric cANGPTL4 has been reported to mediate angiogenesis and inflammation), ANGPTL4 has been implicated to be an ideal environmental sensor. As such, the dysregulation of ANGPTL4 has been described in literature to influence the outcome of a variety of disease models varying from cancer metastasis, atherosclerosis, metabolic syndrome and acute inflammation in association to type II diabetes. 
Aberrant energy metabolism leading up to the accumulation of excess fat in the body through nutritional and hormonal imbalance have also been under scrutiny as a cause for the rising global obesity trends. Recent findings suggest apart from conferring an immunological advantage, the gastrointestinal microbial community might be responsible to modulate host energy metabolism and disease susceptibility. Metabolomics has also spearheaded efforts in identifying the plethora of microbial metabolites and their effects on diseases and health [268]. In addition, nANGPTL4 mediates both glucose and lipid metabolism, suggesting that signaling crosstalk between the host and its commensal microbial community through ANGPTL4 remains to be investigated.

Similar to the dysregulation of the inflammatory landscape during acute colonic inflammation, dietary fat like stearic acid could pose eminent health issues not limiting to inflammatory responses. Generally, common fat found in diet can be classified into saturated, unsaturated and trans fat categories. Using correlation studies, Corwin et al reported that saturated fat intake was inversely associated with bone mineral density [269] while MartínezRamírez et al demonstrated that increased polyunstaturated fat intake increases the predisposition of osteoporotic fractures in elderly patients [270]. However, the complexity involved in categorizing the type of dietary fat intake in human subjects complicates data collection and might explain for the lack of literature.

As a pilot study, we intend to delineate possible differences between different fat types and its effects on bone homeostasis. We explored the effects of the various C18 fat molecules: stearic acid (SA; saturated), oleic acid (OA; non-saturated) and elaidic acid (EA; trans). Using different sources of fat as a model to understand how the excess intake of fat can affect other bone homeostasis, we briefly challenged age-matched mice to $15 \%$ fat: $85 \%$ grounded chow (w/w) for 8 days and recorded for any morphological alterations on the tibia. Scanning electron micrographs reveal that the tibia of fat-challenged mice exhibited a range of depressions and grooves on the bone surface, while the bone matrix formed irregular pits when compared to mice on normal diet (Figure 5.2a). Preliminary findings reveal that connective tissue disorders, organismal injury and abnormalities as well as skeletal and muscular disorders are amongst the top disease functions mapped for fat challenge. Interestingly, we observed a brief increase in TNF and TGF $\beta 1$ expression in both EA and SA but not OA challenge (Figure 5.2b), suggesting that both an increase in trans and saturated fat intake might attribute to the chronic inflammatory phenotype associated to high fat diet observed in several population-based correlative studies. We also observed that the most significant disease associated to an increase in fat diet relates to bone homeostasis: osteoporosis, abnormal bone density and size, as well as skeletal and connective tissue morphology. 
a

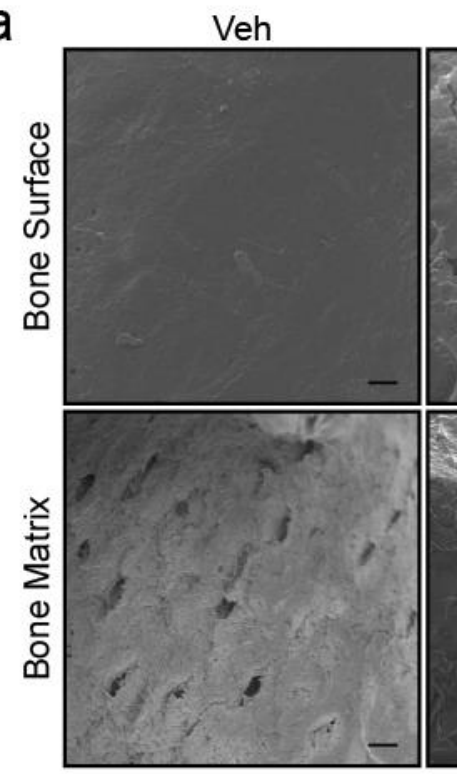

b

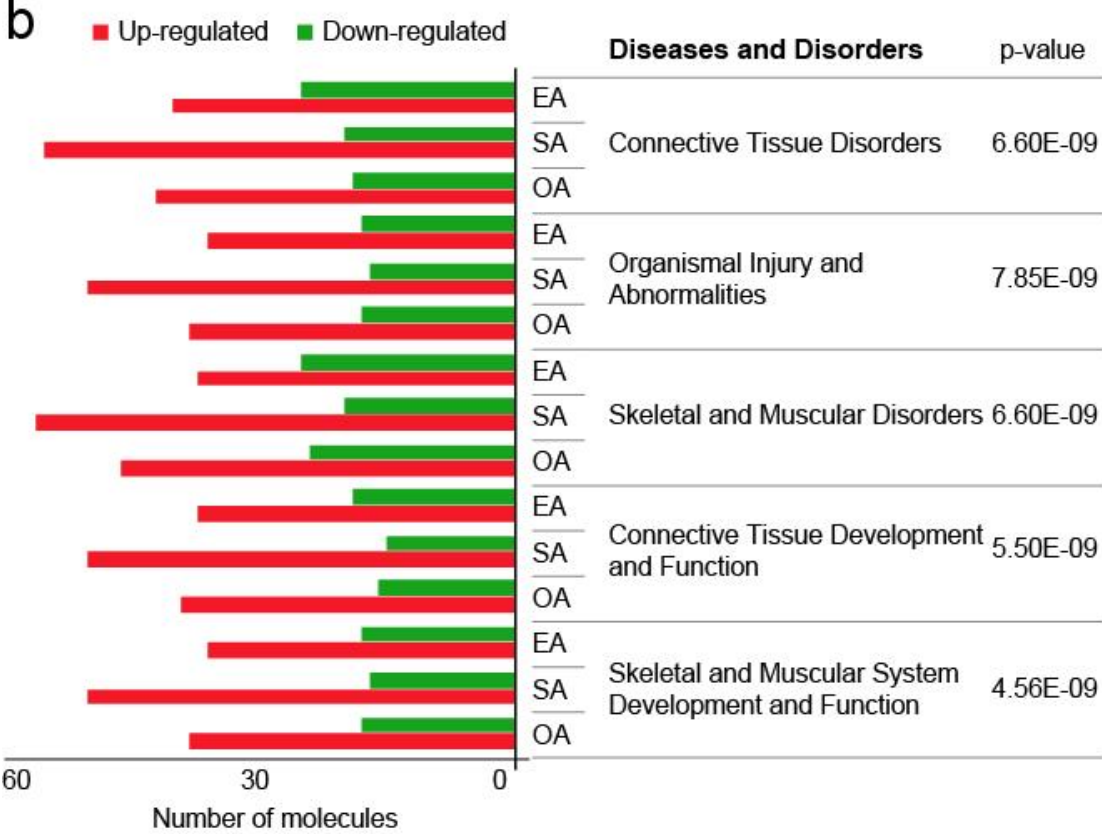

SA

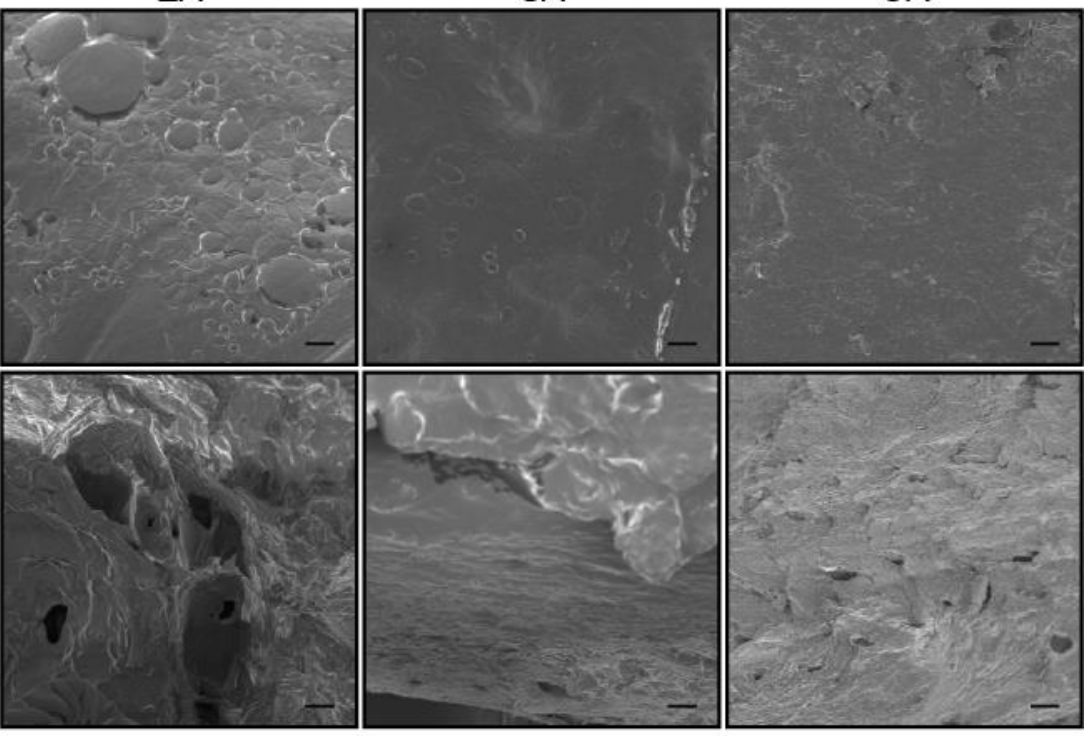

OA 
a disease characterized by decreased bone density, mass and strength, causing skeletal fragility. Since the density of bone mass depended on a fine balance between bone formation by osteoblasts and bone resorption by osteoclasts, this observation suggests a potential homeostatic loop between the bone-adipose axis. To date, Gomez-Ambrosi et al suggested an active glucose homeostasis crosstalk through proteins like osteocalcin and osteopontin that are secreted by bone cells that could impact adipocyte signaling by augmenting circulating adiponectin and insulin $[275,276]$. However, intimate signaling pathways within the boneadipose axis remains to be elucidated.

Quite recently, Shafik et al and Li et al reported that ANGPTL4 exerts its effects through IL$1 \beta$ and NF- $\kappa B$ in breast cancer [277], as well as through IL-6-Stat3 signaling in influenza [229]. However, the identities of other regulatory proteins involved in the ANGPTL4dependent signaling axis remains to be elucidated. On the other hand, independent groups have previously characterized TTP for its role in regulating mRNA transcript stability of a subset of cytokines, and so, have extensively demonstrated that TTP acts as a molecular switch to allow cells to respond quickly to external stimuli during acute inflammation. Despite the wealth of accumulated literature generated by independent laboratories, there has not been much known between environmental sensor proteins like ANGPTL4 and an intermediate, fast-response switch like TTP that has been implicated in the plethora of diseases.

TAMs in close proximity of the pro-inflammatory tumor microenvironment are believed to play key roles in incubating the growing tumor [278, 279]. The plasticity of macrophages allows them to be further polarized into pro-inflammatory M1 or anti-inflammatory M2 states as a response to environmental stimuli $[280,281]$. However, it was reported that TAMs predominantly adopt M2 polarized states [282, 283], promoting a muted immune response essential for immune-suppression around the tumor site. Interestingly, Schumann et al demonstrated that TAMs express high levels of ANGPTL4 [284] while Schmid et al suggested that patients with elevated C-reactive protein (CRP) levels had a worsened prognosis [285]. Wu et al, Waschki et al and Tjeerdema et al later found that ANGPTL4 share a close relationship with CRP during chronic obstructive pulmonary disease [286, 287] and type II diabetes [230]. Hersoug et al also reported that gut microbial-derived LPS increases chylomicron transport in the lymph circulation, promoting fat absorption and also macrophage M2 to M1 polarization [288]. However, the relationship between ANGPTL4 and macrophage polarization involved in tumor proliferation, migration and the EMT process remains largely unclear.

In conclusion, novel findings brought forth in this thesis describes the multi-faceted role of ANGPTL4, drawing links between the external environment, commensal microbiota, host metabolic and immune systems that determine the parameters for health or illness. 
6 ACKNOWLEDGEMENTS 
To set out on a journey following a precise trail towards a destination might be a logical thing that most people do. Although this happens only once in a blue moon in the field of research, I am fortunate to have met some of the greatest people that helped pave the way leading up to this dissertation. This scientific union was made possible by the best supervisors, mentors and colleagues from both Nanyang Technological University (Singapore) and Karolinska Institutet (Sweden).

First and foremost, I would like to express my heartfelt gratitude to Assoc Professor Andrew Tan Nguan Soon of NTU School of Biological Sciences (Singapore), for mentoring me through my doctoral student years, as well as for his generosity, freedom and trust that he bestowed on me. It is almost impossible to not want to push for more data, because his keen eye for detail almost always led to surprises around the corner. His enthusiasm in achieving scientific excellence morphed seemingly preliminary findings into interdisciplinary coalitions, often leading to multipartite collaborations.

I would like to thank Docent Velmurugesan Arulampalam of $\mathrm{KI}$ Institutionen för mikrobiologi, tumör- och cellbiologi (Sweden), for his patience and time during my years over at Stockholm. It was a continuous effort to ensure I didn't slip through the crevice, and to want to speak with me even in the busiest of days. I probably would not have survived my term at $\mathrm{KI}$ without his supervision.

In addition, I would like to thank Professor Sven Pettersson for his guidance over at KI. If only we had more time to explore unknown territories, I anticipate a more complete collaboration could be born out of this NTU-KI affiliation.

Not many will understand how instrumental the role that workmates play in the work that he gets to call his own. Many thanks to the great people working, or used to be working, at the ATAN lab in NTU. I would like to thank Tan Ming Jie, for his contributions and assistance to troubleshooting my experiments. Dr Tan Chek Kun, my second but most probably the most noteworthy mentor in research, I thank him for being a constant pain and for setting targets so high at times, or I would not have grown and learnt the ropes so quickly. To the SBJ Trio - Dr Sng Ming Keat, Dr Teo Ziqiang and Jeremy Chan - life would be so mundane without the trio because they made the long days at lab colorful. Eddie Tan, the occasional diplomat and IT expert, thank you for everything. Maegan Lim, hang in there. I'm not tipping the balance but hey, you're almost done! Mark Tan, enjoy your "eaasssy" life. The newest addition to the big family, Jonathan Wee, your expertise on this last bit of experiments saved me from the trenches.

To Dickson Chee, the most notable of all my students, you were my Sparta and ninja. If we've had more time together, I couldn't imagine the mountains we would have scaled collectively. Koh Hong Zheng, my almost-adopted son, thank you for standing by me and not settling for less! Glendon Phua - who represented an entity of enthusiasm and vigor - 
hey our surname isn't that uncommon afterall! And to my multitasking coolie and commando, Alex Li - you survived my boot camp! Now it's time for you to find your place in this circle of life. I would also like to extend my salutations to my fellow colleagues, past and present: Dr Zhu Pengcheng, Dr Kelvin Chong, Dr Carol Tan, Dr Tan Zhen Wei, Dr Li Liang, Selin Foo, Chen Jia Peng, Wendy Phua, Choo Chee Chong, Justin Lee and Yip Yun Sheng for their time, support, guidance and assistance.

On the other side of the globe, I wish to extent my heartfelt appreciation to my colleagues and staff whom I used to work with at the SP lab in KI. To Dr Viorica Braniste, who was both a friend and a mentor, I miss the dinners we spent closeted together. I am grateful that we met, and for all she's done for me. To this, even "a great pleasure" seems to be an understatement. To Dr Agata Korecka, who has been of great help and a source of guidance, I thank her for assistance and patience in showing me the ropes. I do hope Sligo gets to meet Snow one day. To Dr Maha Al-Asmakh, I admire her for her tolerance and patience with me when she had to sit by the radiator when I insisted in keeping the office windows open during the dead of winter. In her own words, "Never lose faith". To Dr Chng Song Hui, the unexpected friend from an unexpected land and that fellow Singaporean I never met until Stockholm came and almost went, I hope you're enjoying your new postdoctoral life. To Kyaw Soe Oo, I miss those beautiful memories of our weekend brunches and drinking nights. To Dr Lee Changhan, I thank him for forging the unexpected friendship outside the SP lab, and for the wondrous meals we've had together. I would like to acknowledge the following academics: Annika Samuelsson and Johanna Aspsäter from the KI-CFGR team as well as Afrouz Abbaspour and Dr Shawon Lahiri for accomplished contributions to the success of manuscripts leading to the development of this thesis. It has been a privilege and pleasure alongside all of you.

To Dr Said Eshaghi, I thank him for his guidance and for cementing my faith in Science during those undergraduate days I spent in his laboratory. If it wasn't for him, I probably would not have even dreamt that I could make it here. And not forgetting Dr Albert Guskov of Rijksuniversiteit Groningen (Netherlands), who has made even the darkest of days seem a little brighter. And to Gladys Hoh who has not only been a friend but also a confidante, thank you for all the beautiful memories. This doctorate degree would not have been vaguely possible if not for their encouragement.

I would like to thank Dr Philippe Gerard of Institut National de la Recherche Agronomique (INRA, France), for the great memories during his stopover at Stockholm. It was one of those few unforgettable discussions that I would carry with me for a long time. Thank you for the encouragement all these years.

Friends are not forgotten either. To Milton Kwek, who once said he was going to be late for life if he does a doctorate degree. I wonder what he has to say to this now. To Ngoh Xinyi, 
Toh Yifan, Wong Yue Ying and Grace Wong, thank you all for being there and still going strong! I know 12 years is a long time, but what's a decade compared to a friendship of a lifetime? So here's my million-dollar question: when are we all going to congregate in Singapore so we could go for dinner again? To the crazy people who shaped varsity life: Harvey Huang, Gan Xinli and Jacqueline Ng. Thank you for your constructive competition and those cherished memories we forged together; also not forgetting that subtle encouragement when we're each at our limits, and that we can still inch a little bit further. To Elham Zare and Ulrich Amstutz, thank you both for years of unyielding friendship and for keeping Switzerland warmer than it should be. And finally to Matthew Keats, who belonged to a very different world from our world of Research, thank you for sharing a part of your life with me. I kept our crabs alive and I'm sure there is more to destiny than "Kia ora".

On a personal note, I would like to thank Felis Lim: the mother, the authoritarian, the strategist, my audience and partner-in-crime. She has been my pillar of strength and inspiration. She would always be there for me during the bleakest of hours and to brave the next storms. Thank you for everything. I might not have owned the world yet, but to have you will be suffice. And to Snow, whose smile can melt even the coldest of hearts, thank you for not gnawing up the pages of my manuscripts and thesis. I am sure you know that you represent the world to me. To my extended family and friends whom have helped me in one way or another, I thank you all for your company and support. Let's scale greater heights together.

I would like to thank Nanyang Technological University and Karolinska Institutet for awarding me an opportunity at this NTU-KI Double PhD Program, as well as the NTU Research Scholarship for funding my doctoral studies. Last but not least, I would like to thank the efficient people at the administration that made this collaboration possible: Eugene Lee, May Chong and Raymond Lee from NTU-SBS, as well as their counterparts Anita Wallentin and Åsa Belin from KI-MTC. 


\section{REFERENCES}


1. Guarner, F. and J.R. Malagelada, Gut flora in health and disease. Lancet, 2003. 361(9356): p. 512-9.

2. $\quad$ Sears, C.L., A dynamic partnership: celebrating our gut flora. Anaerobe, 2005. 11(5): p. 247-51.

3. Jandhyala, S.M., et al., Role of the normal gut microbiota. World J Gastroenterol, 2015. 21(29): p. 8787-803.

4. Quigley, E.M., Gut bacteria in health and disease. Gastroenterol Hepatol (N Y), 2013. 9(9): p. 560-9.

5. Vedantam, G. and D.W. Hecht, Antibiotics and anaerobes of gut origin. Curr Opin Microbiol, 2003. 6(5): p. 457-61.

6. Schrezenmeir, J. and M. de Vrese, Probiotics, prebiotics, and synbiotics-approaching a definition. Am J Clin Nutr, 2001. 73(2 Suppl): p. 361S-364S.

7. Savage, D.C., Associations and physiological interactions of indigenous microorganisms and gastrointestinal epithelia. Am J Clin Nutr, 1972. 25(12): p. 1372-9.

8. Beaugerie, L. and J.C. Petit, Microbial-gut interactions in health and disease. Antibiotic-associated diarrhoea. Best Pract Res Clin Gastroenterol, 2004. 18(2): p. 337-52.

9. Johansson, M.E., J.M. Larsson, and G.C. Hansson, The two mucus layers of colon are organized by the MUC2 mucin, whereas the outer layer is a legislator of hostmicrobial interactions. Proc Natl Acad Sci U S A, 2011. 108 Suppl 1: p. 4659-65.

10. Bevins, C.L. and N.H. Salzman, Paneth cells, antimicrobial peptides and maintenance of intestinal homeostasis. Nat Rev Microbiol, 2011. 9(5): p. 356-68.

11. Dann, S.M. and L. Eckmann, Innate immune defenses in the intestinal tract. Curr Opin Gastroenterol, 2007. 23(2): p. 115-20.

12. Turner, J.R., Intestinal mucosal barrier function in health and disease. Nat Rev Immunol, 2009. 9(11): p. 799-809.

13. Chacko, A. and J.H. Cummings, Nitrogen losses from the human small bowel: obligatory losses and the effect of physical form of food. Gut, 1988. 29(6): p. 809-15.

14. Cummings, J.H. and G.T. Macfarlane, The control and consequences of bacterial fermentation in the human colon. J Appl Bacteriol, 1991. 70(6): p. 443-59.

15. Parker, D.S., The measurement of production rates of volatile fatty acids in the caecum of the conscious rabbit. Br J Nutr, 1976. 36(1): p. 61-70.

16. Bergman, E.N., Energy contributions of volatile fatty acids from the gastrointestinal tract in various species. Physiol Rev, 1990. 70(2): p. 567-90.

17. Macfarlane, S. and G.T. Macfarlane, Regulation of short-chain fatty acid production. Proc Nutr Soc, 2003. 62(1): p. 67-72.

18. Offermanns, S., Free fatty acid (FFA) and hydroxy carboxylic acid (HCA) receptors. Annu Rev Pharmacol Toxicol, 2014. 54: p. 407-34.

19. Blad, C.C., C. Tang, and S. Offermanns, G protein-coupled receptors for energy metabolites as new therapeutic targets. Nat Rev Drug Discov, 2012. 11(8): p. 603-19. 
20. Stappenbeck, T.S., L.V. Hooper, and J.I. Gordon, Developmental regulation of intestinal angiogenesis by indigenous microbes via Paneth cells. Proc Natl Acad Sci U S A, 2002.99(24): p. 15451-5.

21. Gupta, R., N. Gupta, and P. Rathi, Bacterial lipases: an overview of production, purification and biochemical properties. Appl Microbiol Biotechnol, 2004. 64(6): p. 763-81.

22. Korecka, A., et al., ANGPTL4 expression induced by butyrate and rosiglitazone in human intestinal epithelial cells utilizes independent pathways. Am J Physiol Gastrointest Liver Physiol, 2013. 304(11): p. G1025-37.

23. Ahmed, K., S. Tunaru, and S. Offermanns, GPR109A, GPR109B and GPR81, a family of hydroxy-carboxylic acid receptors. Trends Pharmacol Sci, 2009. 30(11): p. 557-62.

24. Brown, A.J., et al., The Orphan G protein-coupled receptors GPR41 and GPR43 are activated by propionate and other short chain carboxylic acids. J Biol Chem, 2003. 278(13): p. 11312-9.

25. Le Poul, E., et al., Functional characterization of human receptors for short chain fatty acids and their role in polymorphonuclear cell activation. J Biol Chem, 2003. 278(28): p. 25481-9.

26. Cousens, L.S., D. Gallwitz, and B.M. Alberts, Different accessibilities in chromatin to histone acetylase. J Biol Chem, 1979. 254(5): p. 1716-23.

27. Furusawa, Y., et al., Commensal microbe-derived butyrate induces the differentiation of colonic regulatory T cells. Nature, 2013. 504(7480): p. 446-50.

28. Remely, M., et al., Effects of short chain fatty acid producing bacteria on epigenetic regulation of FFAR3 in type 2 diabetes and obesity. Gene, 2014. 537(1): p. 85-92.

29. Cummings, J.H., et al., Short chain fatty acids in human large intestine, portal, hepatic and venous blood. Gut, 1987. 28(10): p. 1221-7.

30. Hong, Y.H., et al., Acetate and propionate short chain fatty acids stimulate adipogenesis via GPCR43. Endocrinology, 2005. 146(12): p. 5092-9.

31. Kimura, I., et al., The gut microbiota suppresses insulin-mediated fat accumulation via the short-chain fatty acid receptor GPR43. Nat Commun, 2013. 4: p. 1829.

32. Ang, Z., et al., Human and mouse monocytes display distinct signalling and cytokine profiles upon stimulation with FFAR2/FFAR3 short-chain fatty acid receptor agonists. Sci Rep, 2016. 6: p. 34145.

33. Backhed, F., et al., The gut microbiota as an environmental factor that regulates fat storage. Proc Natl Acad Sci U S A, 2004. 101(44): p. 15718-23.

34. Mattijssen, F., et al., Angptl4 serves as an endogenous inhibitor of intestinal lipid digestion. Mol Metab, 2014. 3(2): p. 135-44.

35. Lukovac, S., et al., Differential modulation by Akkermansia muciniphila and Faecalibacterium prausnitzii of host peripheral lipid metabolism and histone acetylation in mouse gut organoids. MBio, 2014. 5(4).

36. Brennan, A.M. and C.S. Mantzoros, Drug Insight: the role of leptin in human physiology and pathophysiology--emerging clinical applications. Nat Clin Pract Endocrinol Metab, 2006. 2(6): p. 318-27. 
37. Saltiel, A.R. and C.R. Kahn, Insulin signalling and the regulation of glucose and lipid metabolism. Nature, 2001. 414(6865): p. 799-806.

38. Backhed, F., et al., Mechanisms underlying the resistance to diet-induced obesity in germ-free mice. Proc Natl Acad Sci U S A, 2007. 104(3): p. 979-84.

39. Skurk, T., et al., Relationship between adipocyte size and adipokine expression and secretion. J Clin Endocrinol Metab, 2007. 92(3): p. 1023-33.

40. Samuel, B.S., et al., Effects of the gut microbiota on host adiposity are modulated by the short-chain fatty-acid binding G protein-coupled receptor, Gpr41. Proc Natl Acad Sci U S A, 2008. 105(43): p. 16767-72.

41. Cuche, G., J.C. Cuber, and C.H. Malbert, Ileal short-chain fatty acids inhibit gastric motility by a humoral pathway. Am J Physiol Gastrointest Liver Physiol, 2000. 279(5): p. G925-30.

42. Turnbaugh, P.J., et al., An obesity-associated gut microbiome with increased capacity for energy harvest. Nature, 2006. 444(7122): p. 1027-31.

43. Turnbaugh, P.J., et al., The effect of diet on the human gut microbiome: a metagenomic analysis in humanized gnotobiotic mice. Sci Transl Med, 2009. 1(6): p. 6ra14.

44. Parekh, P.J., et al., The role and influence of gut microbiota in pathogenesis and management of obesity and metabolic syndrome. Front Endocrinol (Lausanne), 2014. 5: p. 47.

45. Parekh, P.J., L.A. Balart, and D.A. Johnson, The Influence of the Gut Microbiome on Obesity, Metabolic Syndrome and Gastrointestinal Disease. Clin Transl Gastroenterol, 2015. 6: p. e91.

46. Ley, R.E., et al., Obesity alters gut microbial ecology. Proc Natl Acad Sci U S A, 2005. 102(31): p. 11070-5.

47. Daniel, H., et al., High-fat diet alters gut microbiota physiology in mice. ISME J, 2014. 8(2): p. 295-308.

48. Ohkusa, T. and S. Koido, Intestinal microbiota and ulcerative colitis. J Infect Chemother, 2015. 21(11): p. 761-8.

49. Almagro-Moreno, S., K. Pruss, and R.K. Taylor, Intestinal Colonization Dynamics of Vibrio cholerae. PLoS Pathog, 2015. 11(5): p. e1004787.

50. Smoot, D.T., How does Helicobacter pylori cause mucosal damage? Direct mechanisms. Gastroenterology, 1997. 113(6 Suppl): p. S31-4; discussion S50.

51. Abreu, M.T., Toll-like receptor signalling in the intestinal epithelium: how bacterial recognition shapes intestinal function. Nat Rev Immunol, 2010. 10(2): p. 131-44.

52. Takeuchi, O. and S. Akira, Pattern recognition receptors and inflammation. Cell, 2010. 140(6): p. 805-20.

53. Favier, C.F., et al., Molecular monitoring of succession of bacterial communities in human neonates. Appl Environ Microbiol, 2002. 68(1): p. 219-26.

54. Martin, R., et al., Early-Life Events, Including Mode of Delivery and Type of Feeding, Siblings and Gender, Shape the Developing Gut Microbiota. PLoS One, 2016. 11(6): p. e0158498. 
55. Gronlund, M.M., et al., Fecal microflora in healthy infants born by different methods of delivery: permanent changes in intestinal flora after cesarean delivery. J Pediatr Gastroenterol Nutr, 1999. 28(1): p. 19-25.

56. Bettelheim, K.A., et al., The origin of $O$ serotypes of Escherichia coli in babies after normal delivery. J Hyg (Lond), 1974. 72(1): p. 67-70.

57. Flak, M.B., J.F. Neves, and R.S. Blumberg, Immunology. Welcome to the microgenderome. Science, 2013. 339(6123): p. 1044-5.

58. Carman, R.J., et al., Ciprofloxacin at low levels disrupts colonization resistance of human fecal microflora growing in chemostats. Regul Toxicol Pharmacol, 2004. 40(3): p. 319-26.

59. Olszak, T., et al., Microbial exposure during early life has persistent effects on natural killer T cell function. Science, 2012. 336(6080): p. 489-93.

60. Wu, G.D., et al., Linking long-term dietary patterns with gut microbial enterotypes. Science, 2011. 334(6052): p. 105-8.

61. Arvidsson, C., A. Hallen, and F. Backhed, Generating and Analyzing Germ-Free Mice. Curr Protoc Mouse Biol, 2012. 2(4): p. 307-16.

62. Inagaki, H., et al., Increased susceptibility to primary infection with Listeria monocytogenes in germfree mice may be due to lack of accumulation of L-selectin+ CD44+ T cells in sites of inflammation. Infect Immun, 1996. 64(8): p. 3280-7.

63. Diamond, B., et al., It takes guts to grow a brain: Increasing evidence of the important role of the intestinal microflora in neuro- and immune-modulatory functions during development and adulthood. Bioessays, 2011. 33(8): p. 588-91.

64. Yamamoto, M., et al., A microarray analysis of gnotobiotic mice indicating that microbial exposure during the neonatal period plays an essential role in immune system development. BMC Genomics, 2012. 13: p. 335.

65. Al-Asmakh, M., et al., The gut microbiota and developmental programming of the testis in mice. PLoS One, 2014. 9(8): p. e103809.

66. Braniste, V., et al., The gut microbiota influences blood-brain barrier permeability in mice. Sci Transl Med, 2014. 6(263): p. 263 ra158.

67. Krajmalnik-Brown, R., et al., Effects of gut microbes on nutrient absorption and energy regulation. Nutr Clin Pract, 2012. 27(2): p. 201-14.

68. Diaz Heijtz, R., et al., Normal gut microbiota modulates brain development and behavior. Proc Natl Acad Sci U S A, 2011. 108(7): p. 3047-52.

69. Cebra, J.J., Influences of microbiota on intestinal immune system development. Am J Clin Nutr, 1999. 69(5): p. 1046S-1051S.

70. Rhee, K.J., et al., Role of commensal bacteria in development of gut-associated lymphoid tissues and preimmune antibody repertoire. J Immunol, 2004. 172(2): p. 1118-24.

71. Abrams, G.D., H. Bauer, and H. Sprinz, Influence of the normal flora on mucosal morphology and cellular renewal in the ileum. A comparison of germ-free and conventional mice. Lab Invest, 1963. 12: p. 355-64. 
72. Pull, S.L., et al., Activated macrophages are an adaptive element of the colonic epithelial progenitor niche necessary for regenerative responses to injury. Proc Natl Acad Sci U S A, 2005. 102(1): p. 99-104.

73. Slack, E., et al., Innate and adaptive immunity cooperate flexibly to maintain hostmicrobiota mutualism. Science, 2009. 325(5940): p. 617-20.

74. Yu, Q., et al., MyD88-dependent signaling for IL-15 production plays an important role in maintenance of CD8 alpha alpha TCR alpha beta and TCR gamma delta intestinal intraepithelial lymphocytes. J Immunol, 2006. 176(10): p. 6180-5.

75. Carvalho, F.A., et al., Toll-like receptor-gut microbiota interactions: perturb at your own risk! Annu Rev Physiol, 2012. 74: p. 177-98.

76. Waldner, M.J. and M.F. Neurath, Colitis-associated cancer: the role of T cells in tumor development. Semin Immunopathol, 2009. 31(2): p. 249-56.

77. Saraggi, D., et al., The molecular landscape of colitis-associated carcinogenesis. Dig Liver Dis, 2017. 49(4): p. 326-330.

78. Rossin, D., et al., HNE and cholesterol oxidation products in colorectal inflammation and carcinogenesis. Free Radic Biol Med, 2017.

79. Du, L., et al., KRAS and TP53 mutations in inflammatory bowel disease-associated colorectal cancer: a meta-analysis. Oncotarget, 2017.

80. de Martel, C. and S. Franceschi, Infections and cancer: established associations and new hypotheses. Crit Rev Oncol Hematol, 2009. 70(3): p. 183-94.

81. Taylor, G.A., et al., A pathogenetic role for TNF alpha in the syndrome of cachexia, arthritis, and autoimmunity resulting from tristetraprolin (TTP) deficiency. Immunity, 1996. 4(5): p. 445-54.

82. Carballo, E., W.S. Lai, and P.J. Blackshear, Feedback inhibition of macrophage tumor necrosis factor-alpha production by tristetraprolin. Science, 1998. 281(5379): p. 1001-5.

83. Kontoyiannis, D., et al., Impaired on/off regulation of TNF biosynthesis in mice lacking TNF AU-rich elements: implications for joint and gut-associated immunopathologies. Immunity, 1999. 10(3): p. 387-98.

84. Lai, W.S., et al., Evidence that tristetraprolin binds to AU-rich elements and promotes the deadenylation and destabilization of tumor necrosis factor alpha mRNA. Mol Cell Biol, 1999. 19(6): p. 4311-23.

85. Carballo, E., W.S. Lai, and P.J. Blackshear, Evidence that tristetraprolin is a physiological regulator of granulocyte-macrophage colony-stimulating factor messenger RNA deadenylation and stability. Blood, 2000. 95(6): p. 1891-9.

86. Smoak, K. and J.A. Cidlowski, Glucocorticoids regulate tristetraprolin synthesis and posttranscriptionally regulate tumor necrosis factor alpha inflammatory signaling. Mol Cell Biol, 2006. 26(23): p. 9126-35.

87. Sanduja, S., et al., The role of tristetraprolin in cancer and inflammation. Front Biosci (Landmark Ed), 2012. 17: p. 174-88.

88. Sauer, I., et al., Interferons limit inflammatory responses by induction of tristetraprolin. Blood, 2006. 107(12): p. 4790-7. 
89. Marderosian, M., et al., Tristetraprolin regulates Cyclin D1 and c-Myc mRNA stability in response to rapamycin in an Akt-dependent manner via p38 MAPK signaling. Oncogene, 2006. 25(47): p. 6277-90.

90. Ogilvie, R.L., et al., Tristetraprolin mediates interferon-gamma mRNA decay. J Biol Chem, 2009. 284(17): p. 11216-23.

91. Fechir, M., et al., Tristetraprolin regulates the expression of the human inducible nitric-oxide synthase gene. Mol Pharmacol, 2005. 67(6): p. 2148-61.

92. Sen, R. and D. Baltimore, Multiple nuclear factors interact with the immunoglobulin enhancer sequences. Cell 1986. 46: 705-716. J Immunol, 2006. 177(11): p. 7485-96.

93. Hayden, M.S. and S. Ghosh, Regulation of NF-kappaB by TNF family cytokines. Semin Immunol, 2014. 26(3): p. 253-66.

94. Hinz, M., S.C. Arslan, and C. Scheidereit, It takes two to tango: IkappaBs, the multifunctional partners of NF-kappaB. Immunol Rev, 2012. 246(1): p. 59-76.

95. Henkel, T., et al., Rapid proteolysis of I kappa B-alpha is necessary for activation of transcription factor NF-kappa B. Nature, 1993. 365(6442): p. 182-5.

96. Ghosh, S. and D. Baltimore, Activation in vitro of NF-kappa B by phosphorylation of its inhibitor I kappa B. Nature, 1990. 344(6267): p. 678-82.

97. Mitchell, S., J. Vargas, and A. Hoffmann, Signaling via the NFkappaB system. Wiley Interdiscip Rev Syst Biol Med, 2016. 8(3): p. 227-41.

98. Liang, J., et al., RNA-destabilizing factor tristetraprolin negatively regulates NFkappaB signaling. J Biol Chem, 2009. 284(43): p. 29383-90.

99. Schichl, Y.M., et al., Tristetraprolin impairs NF-kappaB/p65 nuclear translocation. J Biol Chem, 2009. 284(43): p. 29571-81.

100. Lai, W.S., D.J. Stumpo, and P.J. Blackshear, Rapid insulin-stimulated accumulation of an mRNA encoding a proline-rich protein. J Biol Chem, 1990. 265(27): p. 1655663.

101. DuBois, R.N., et al., A growth factor-inducible nuclear protein with a novel cysteine/histidine repetitive sequence. J Biol Chem, 1990. 265(31): p. 19185-91.

102. Blackshear, P.J., et al., Zfp36l3, a rodent X chromosome gene encoding a placentaspecific member of the Tristetraprolin family of CCCH tandem zinc finger proteins. Biol Reprod, 2005. 73(2): p. 297-307.

103. Blackshear, P.J., et al., Characteristics of the interaction of a synthetic human tristetraprolin tandem zinc finger peptide with AU-rich element-containing RNA substrates. J Biol Chem, 2003. 278(22): p. 19947-55.

104. Lai, W.S., et al., Promoter analysis of Zfp-36, the mitogen-inducible gene encoding the zinc finger protein tristetraprolin. J Biol Chem, 1995. 270(42): p. 25266-72.

105. Florkowska, M., et al., EGF activates TTP expression by activation of ELK-1 and EGR-1 transcription factors. BMC Mol Biol, 2012. 13: p. 8.

106. Taylor, G.A., et al., Mitogens stimulate the rapid nuclear to cytosolic translocation of tristetraprolin, a potential zinc-finger transcription factor. Mol Endocrinol, 1996. 10(2): p. 140-6. 
107. DuBois, R.N., et al., Transforming growth factor alpha regulation of two zinc fingercontaining immediate early response genes in intestine. Cell Growth Differ, 1995. 6(5): p. 523-9.

108. Tchen, C.R., et al., The stability of tristetraprolin $m R N A$ is regulated by mitogenactivated protein kinase $p 38$ and by tristetraprolin itself. J Biol Chem, 2004. 279(31): p. 32393-400.

109. Marchese, F.P., et al., MAPKAP kinase 2 blocks tristetraprolin-directed mRNA decay by inhibiting CAF1 deadenylase recruitment. J Biol Chem, 2010. 285(36): p. 27590600 .

110. Sun, L., et al., Tristetraprolin (TTP)-14-3-3 complex formation protects TTP from dephosphorylation by protein phosphatase $2 a$ and stabilizes tumor necrosis factoralpha mRNA. J Biol Chem, 2007. 282(6): p. 3766-77.

111. Clement, S.L., et al., Phosphorylation of tristetraprolin by MK2 impairs AU-rich element mRNA decay by preventing deadenylase recruitment. Mol Cell Biol, 2011. 31(2): p. 256-66.

112. Qian, F., et al., Pivotal Role of Mitogen-Activated Protein Kinase-Activated Protein Kinase 2 in Inflammatory Pulmonary Diseases. Curr Protein Pept Sci, 2016. 17(4): p. 332-42.

113. Nadar, M., et al., HuR binding to AU-rich elements present in the 3' untranslated region of Classical swine fever virus. Virol J, 2011. 8: p. 340.

114. $\mathrm{Wu}, \mathrm{X}$., et al., Identification and validation of novel small molecule disruptors of HuR-mRNA interaction. ACS Chem Biol, 2015. 10(6): p. 1476-84.

115. Nabors, L.B., et al., Tumor necrosis factor alpha induces angiogenic factor upregulation in malignant glioma cells: a role for RNA stabilization and HuR. Cancer Res, 2003. 63(14): p. 4181-7.

116. Guo, X., et al., MicroRNA-16 modulates HuR regulation of cyclin E1 in breast cancer cells. Int J Mol Sci, 2015. 16(4): p. 7112-32.

117. DeMicco, A., et al., B Cell-Intrinsic Expression of the HuR RNA-Binding Protein Is Required for the T Cell-Dependent Immune Response In Vivo. J Immunol, 2015. 195(7): p. 3449-62.

118. Takeuchi, O., HuR keeps interferon-beta mRNA stable. Eur J Immunol, 2015. 45(5): p. 1296-9.

119. To, K.K., W.W. Leung, and S.S. Ng, Exploiting a novel miR-519c-HuR-ABCG2 regulatory pathway to overcome chemoresistance in colorectal cancer. Exp Cell Res, 2015. 338(2): p. 222-31.

120. Jimbo, M., et al., Targeting the mRNA-binding protein HuR impairs malignant characteristics of pancreatic ductal adenocarcinoma cells. Oncotarget, 2015. 6(29): p. 27312-31.

121. Shwetha, S., et al., HuR Displaces Polypyrimidine Tract Binding Protein To Facilitate La Binding to the 3' Untranslated Region and Enhances Hepatitis C Virus Replication. J Virol, 2015. 89(22): p. 11356-71.

122. Tiedje, C., et al., The p38/MK2-driven exchange between tristetraprolin and HuR regulates AU-rich element-dependent translation. PLoS Genet, 2012. 8(9): p. e1002977. 
123. Tisoncik, J.R., et al., Into the eye of the cytokine storm. Microbiol Mol Biol Rev, 2012. 76(1): p. 16-32.

124. Ulrichs, K., et al., Immunosuppression by cytostatic drugs? Behring Inst Mitt, 1984(74): p. 239-49.

125. Mahmud, N., D. Klipa, and N. Ahsan, Antibody immunosuppressive therapy in solidorgan transplant: Part I. MAbs, 2010. 2(2): p. 148-56.

126. Klipa, D., N. Mahmud, and N. Ahsan, Antibody immunosuppressive therapy in solid organ transplant: Part II. MAbs, 2010. 2(6): p. 607-12.

127. Roy, S., et al., Equilibrium unfolding of cyclophilin from Leishmania donovani: characterization of intermediate states. Int J Biol Macromol, 2014. 69: p. 353-60.

128. Coutinho, A.E. and K.E. Chapman, The anti-inflammatory and immunosuppressive effects of glucocorticoids, recent developments and mechanistic insights. Mol Cell Endocrinol, 2011. 335(1): p. 2-13.

129. Amano, Y., S.W. Lee, and A.C. Allison, Inhibition by glucocorticoids of the formation of interleukin-1 alpha, interleukin-1 beta, and interleukin-6: mediation by decreased mRNA stability. Mol Pharmacol, 1993. 43(2): p. 176-82.

130. Auphan, N., et al., Immunosuppression by glucocorticoids: inhibition of NF-kappa B activity through induction of I kappa B synthesis. Science, 1995. 270(5234): p. 28690.

131. Brewer, J.A., et al., T-cell glucocorticoid receptor is required to suppress COX-2mediated lethal immune activation. Nat Med, 2003. 9(10): p. 1318-22.

132. Lasa, M., et al., Dexamethasone causes sustained expression of mitogen-activated protein kinase (MAPK) phosphatase 1 and phosphatase-mediated inhibition of MAPK p38. Mol Cell Biol, 2002. 22(22): p. 7802-11.

133. Kassel, O., et al., Glucocorticoids inhibit MAP kinase via increased expression and decreased degradation of MKP-1. EMBO J, 2001. 20(24): p. 7108-16.

134. Newton, R., et al., Glucocorticoids inhibit IL-1beta-induced GM-CSF expression at multiple levels: roles for the ERK pathway and repression by MKP-1. Biochem J, 2010. 427(1): p. 113-24.

135. Abraham, S.M., et al., Antiinflammatory effects of dexamethasone are partly dependent on induction of dual specificity phosphatase 1. J Exp Med, 2006. 203(8): p. 1883-9.

136. Prabhala, P., et al., Corticosteroid-Induced MKP-1 Represses Pro-Inflammatory Cytokine Secretion by Enhancing Activity of Tristetraprolin (TTP) in ASM Cells. J Cell Physiol, 2016. 231(10): p. 2153-8.

137. Huotari, N., et al., Regulation of tristetraprolin expression by mitogen-activated protein kinase phosphatase-1. APMIS, 2012. 120(12): p. 988-99.

138. Wells, M.L., L. Perera, and P.J. Blackshear, An Ancient Family of RNA-Binding Proteins: Still Important! Trends Biochem Sci, 2017. 42(4): p. 285-296.

139. Wagner, R.P., Anecdotal, historical and critical commentaries on genetics. Rudolph Virchow and the genetic basis of somatic ecology. Genetics, 1999. 151(3): p. 917-20. 
140. Balkwill, F. and A. Mantovani, Inflammation and cancer: back to Virchow? Lancet, 2001. 357(9255): p. 539-45.

141. Cardesa, A., et al., The Kaiser's cancer revisited: was Virchow totally wrong? Virchows Arch, 2011. 458(6): p. 649-57.

142. Ober, W.B., The case of the Kaiser's cancer. Pathol Annu, 1970. 5: p. 207-16.

143. Katsusaburo Yamagiwa (1863-1930). CA Cancer J Clin, 1977. 27(3): p. 172-3.

144. Kumar, P. and F.A. Murphy, Who is this man? Francis Peyton Rous. Emerg Infect Dis, 2013. 19(4): p. 661-3.

145. Blankenstein, T. and Z. Qin, Chemical carcinogens as foreign bodies and some pitfalls regarding cancer immune surveillance. Adv Cancer Res, 2003. 90: p. 179207.

146. Wogan, G.N., et al., Environmental and chemical carcinogenesis. Semin Cancer Biol, 2004. 14(6): p. 473-86.

147. Liou, G.Y. and P. Storz, Reactive oxygen species in cancer. Free Radic Res, 2010. 44(5): p. 479-96.

148. Otani, H., Reactive oxygen species as mediators of signal transduction in ischemic preconditioning. Antioxid Redox Signal, 2004. 6(2): p. 449-69.

149. Touyz, R.M., Reactive oxygen species as mediators of calcium signaling by angiotensin II: implications in vascular physiology and pathophysiology. Antioxid Redox Signal, 2005. 7(9-10): p. 1302-14.

150. Birben, E., et al., Oxidative stress and antioxidant defense. World Allergy Organ J, 2012. 5(1): p. 9-19.

151. Vatansever, F., et al., Antimicrobial strategies centered around reactive oxygen species--bactericidal antibiotics, photodynamic therapy, and beyond. FEMS Microbiol Rev, 2013. 37(6): p. 955-89.

152. Cadet, J. and J.R. Wagner, DNA base damage by reactive oxygen species, oxidizing agents, and UV radiation. Cold Spring Harb Perspect Biol, 2013. 5(2).

153. Benzi, G. and A. Moretti, Are reactive oxygen species involved in Alzheimer's disease? Neurobiol Aging, 1995. 16(4): p. 661-74.

154. Dumont, M. and M.F. Beal, Neuroprotective strategies involving ROS in Alzheimer disease. Free Radic Biol Med, 2011. 51(5): p. 1014-26.

155. De Maria, N., et al., Association between reactive oxygen species and disease activity in chronic hepatitis $C$. Free Radic Biol Med, 1996. 21(3): p. 291-5.

156. Esrefoglu, M., Oxidative stress and benefits of antioxidant agents in acute and chronic hepatitis. Hepat Mon, 2012. 12(3): p. 160-7.

157. Ames, B.N., Dietary carcinogens and anticarcinogens. Oxygen radicals and degenerative diseases. Science, 1983. 221(4617): p. 1256-64.

158. Zhou, D., L. Shao, and D.R. Spitz, Reactive oxygen species in normal and tumor stem cells. Adv Cancer Res, 2014. 122: p. 1-67.

159. Panieri, E. and M.M. Santoro, ROS homeostasis and metabolism: a dangerous liason in cancer cells. Cell Death Dis, 2016. 7(6): p. e2253. 
160. Gupta, S.C., et al., Upsides and downsides of reactive oxygen species for cancer: the roles of reactive oxygen species in tumorigenesis, prevention, and therapy. Antioxid Redox Signal, 2012. 16(11): p. 1295-322.

161. Karin, M., NF-kappaB as a critical link between inflammation and cancer. Cold Spring Harb Perspect Biol, 2009. 1(5): p. a000141.

162. Hoesel, B. and J.A. Schmid, The complexity of NF-kappaB signaling in inflammation and cancer. Mol Cancer, 2013. 12: p. 86.

163. Tochhawng, L., et al., Redox regulation of cancer cell migration and invasion. Mitochondrion, 2013. 13(3): p. 246-53.

164. Sakurai, H., et al., TAK1-TAB1 fusion protein: a novel constitutively active mitogenactivated protein kinase kinase kinase that stimulates AP-1 and NF-kappaB signaling pathways. Biochem Biophys Res Commun, 2002. 297(5): p. 1277-81.

165. Takaesu, G., et al., TAB2, a novel adaptor protein, mediates activation of TAK1 MAPKKK by linking TAK1 to TRAF6 in the IL-1 signal transduction pathway. Mol Cell, 2000. 5(4): p. 649-58.

166. Shim, J.H., et al., TAK1, but not TAB1 or TAB2, plays an essential role in multiple signaling pathways in vivo. Genes Dev, 2005. 19(22): p. 2668-81.

167. Yang, S., et al., Therapeutic effect of methyl salicylate 2-O-beta-d-lactoside on LPSinduced acute lung injury by inhibiting TAK1/NF-kappaB phosphorylation and NLRP3 expression. Int Immunopharmacol, 2016. 40: p. 219-228.

168. Li, K., et al., TAK1 knockdown enhances lipopolysaccharide-induced secretion of proinflammatory cytokines in myeloid cells via unleashing MEKK3 activity. Cell Immunol, 2016. 310: p. 193-198.

169. Yamaguchi, K., et al., Identification of a member of the MAPKKK family as a potential mediator of TGF-beta signal transduction. Science, 1995. 270(5244): p. 2008-11.

170. Shirakabe, K., et al., TAK1 mediates the ceramide signaling to stress-activated protein kinase/c-Jun N-terminal kinase. J Biol Chem, 1997. 272(13): p. 8141-4.

171. Sun, P., et al., Salidroside Regulates Inflammatory Response in Raw 264.7 Macrophages via TLR4/TAK1 and Ameliorates Inflammation in Alcohol Binge Drinking-Induced Liver Injury. Molecules, 2016. 21(11).

172. Choi, M.E., Y. Ding, and S.I. Kim, TGF-beta signaling via TAK1 pathway: role in kidney fibrosis. Semin Nephrol, 2012. 32(3): p. 244-52.

173. Takatsu, Y., et al., TAK1 participates in c-Jun N-terminal kinase signaling during Drosophila development. Mol Cell Biol, 2000. 20(9): p. 3015-26.

174. Kishimoto, K., K. Matsumoto, and J. Ninomiya-Tsuji, TAK1 mitogen-activated protein kinase kinase kinase is activated by autophosphorylation within its activation loop. J Biol Chem, 2000. 275(10): p. 7359-64.

175. Dey, N., et al., TAK1 regulates NF-KappaB and AP-1 activation in airway epithelial cells following RSV infection. Virology, 2011. 418(2): p. 93-101.

176. Liu, R., et al., HIV-1 Vpr stimulates NF-kappaB and AP-1 signaling by activating TAK1. Retrovirology, 2014. 11: p. 45. 
177. Omori, E., et al., TAK1 regulates reactive oxygen species and cell death in keratinocytes, which is essential for skin integrity. J Biol Chem, 2008. 283(38): p. 26161-8.

178. Onodera, Y., et al., Reactive oxygen species induce Cox-2 expression via TAK1 activation in synovial fibroblast cells. FEBS Open Bio, 2015. 5: p. 492-501.

179. Wang, J.S., et al., TAK1 inhibition-induced RIP1-dependent apoptosis in murine macrophages relies on constitutive TNF-alpha signaling and ROS production. $\mathrm{J}$ Biomed Sci, 2015. 22: p. 76.

180. Morioka, S., et al., TAK1 kinase determines TRAIL sensitivity by modulating reactive oxygen species and cIAP. Oncogene, 2009. 28(23): p. 2257-65.

181. Brindle, N.P., P. Saharinen, and K. Alitalo, Signaling and functions of angiopoietin-1 in vascular protection. Circ Res, 2006. 98(8): p. 1014-23.

182. Maisonpierre, P.C., et al., Angiopoietin-2, a natural antagonist for Tie2 that disrupts in vivo angiogenesis. Science, 1997. 277(5322): p. 55-60.

183. Barton, W.A., D. Tzvetkova, and D.B. Nikolov, Structure of the angiopoietin-2 receptor binding domain and identification of surfaces involved in Tie2 recognition. Structure, 2005. 13(5): p. 825-32.

184. Alves, B.E., et al., Imbalances in serum angiopoietin concentrations are early predictors of septic shock development in patients with post chemotherapy febrile neutropenia. BMC Infect Dis, 2010. 10: p. 143.

185. Quagliarini, F., et al., Atypical angiopoietin-like protein that regulates ANGPTL3. Proc Natl Acad Sci U S A, 2012. 109(48): p. 19751-6.

186. Hato, T., M. Tabata, and Y. Oike, The role of angiopoietin-like proteins in angiogenesis and metabolism. Trends Cardiovasc Med, 2008. 18(1): p. 6-14.

187. Katoh, Y. and M. Katoh, Comparative integromics on Angiopoietin family members. Int J Mol Med, 2006. 17(6): p. 1145-9.

188. Kersten, S., Regulation of lipid metabolism via angiopoietin-like proteins. Biochem Soc Trans, 2005. 33(Pt 5): p. 1059-62.

189. Oike, Y., [Role of angiopoietin-like protein (Angptl)]. Seikagaku, 2005. 77(11): p. 1412-7.

190. Kersten, S., Physiological regulation of lipoprotein lipase. Biochim Biophys Acta, 2014. 1841(7): p. 919-33.

191. Schumacher, A., et al., Angptl4 is upregulated under inflammatory conditions in the bone marrow of mice, expands myeloid progenitors, and accelerates reconstitution of platelets after myelosuppressive therapy. J Hematol Oncol, 2015. 8: p. 64.

192. Ventura Ferreira, M.S., et al., Ex vivo expansion of cord blood-CD34(+) cells using IGFBP 2 and Angptl-5 impairs short-term lymphoid repopulation in vivo. $\mathrm{J}$ Tissue Eng Regen Med, 2013. 7(12): p. 944-54.

193. Parri, M., et al., Angiopoietin-like 7, a novel pro-angiogenetic factor over-expressed in cancer. Angiogenesis, 2014. 17(4): p. 881-96.

194. Kim, K.A., et al., High fat diet-induced gut microbiota exacerbates inflammation and obesity in mice via the TLR4 signaling pathway. PLoS One, 2012. 7(10): p. e47713. 
195. Goh, Y.Y., et al., Angiopoietin-like 4 interacts with integrins betal and beta5 to modulate keratinocyte migration. Am J Pathol, 2010. 177(6): p. 2791-803.

196. Guo, L., et al., Silencing Angiopoietin-Like Protein 4 (ANGPTL4) Protects Against Lipopolysaccharide-Induced Acute Lung Injury Via Regulating SIRT1 /NF-kB Pathway. J Cell Physiol, 2015. 230(10): p. 2390-402.

197. Zhu, P., et al., Angiopoietin-like 4 protein elevates the prosurvival intracellular O2():H2O2 ratio and confers anoikis resistance to tumors. Cancer Cell, 2011. 19(3): p. 401-15.

198. Huang, R.L., et al., ANGPTL4 modulates vascular junction integrity by integrin signaling and disruption of intercellular VE-cadherin and claudin-5 clusters. Blood, 2011. 118(14): p. 3990-4002.

199. Lei, X., et al., Proteolytic processing of angiopoietin-like protein 4 by proprotein convertases modulates its inhibitory effects on lipoprotein lipase activity. J Biol Chem, 2011. 286(18): p. 15747-56.

200. Ge, H., et al., Oligomerization and regulated proteolytic processing of angiopoietinlike protein 4. J Biol Chem, 2004. 279(3): p. 2038-45.

201. Li, H., et al., Hypoxia-inducible factor 1 alpha-activated angiopoietin-like protein 4 contributes to tumor metastasis via vascular cell adhesion molecule-1/integrin betal signaling in human hepatocellular carcinoma. Hepatology, 2011. 54(3): p. 910-9.

202. Zhang, H., et al., HIF-1-dependent expression of angiopoietin-like 4 and L1CAM mediates vascular metastasis of hypoxic breast cancer cells to the lungs. Oncogene, 2012. 31(14): p. 1757-70.

203. Mandard, S., et al., The direct peroxisome proliferator-activated receptor target fasting-induced adipose factor (FIAF/PGAR/ANGPTL4) is present in blood plasma as a truncated protein that is increased by fenofibrate treatment. J Biol Chem, 2004. 279(33): p. 34411-20.

204. Inoue, T., et al., Cross-enhancement of ANGPTL4 transcription by HIF1 alpha and PPAR beta/delta is the result of the conformational proximity of two response elements. Genome Biol, 2014. 15(4): p. R63.

205. Yoon, J.C., et al., Peroxisome proliferator-activated receptor gamma target gene encoding a novel angiopoietin-related protein associated with adipose differentiation. Mol Cell Biol, 2000. 20(14): p. 5343-9.

206. Koliwad, S.K., et al., Angiopoietin-like 4 (ANGPTL4, fasting-induced adipose factor) is a direct glucocorticoid receptor target and participates in glucocorticoid-regulated triglyceride metabolism. J Biol Chem, 2009. 284(38): p. 25593-601.

207. Padua, D., et al., TGFbeta primes breast tumors for lung metastasis seeding through angiopoietin-like 4. Cell, 2008. 133(1): p. 66-77.

208. Bierie, B. and H.L. Moses, Tumour microenvironment: TGFbeta: the molecular Jekyll and Hyde of cancer. Nat Rev Cancer, 2006. 6(7): p. 506-20.

209. Kim, I., et al., Hepatic expression, synthesis and secretion of a novel fibrinogen/angiopoietin-related protein that prevents endothelial-cell apoptosis. Biochem J, 2000. 346 Pt 3: p. 603-10.

210. Grootaert, C., et al., Angiopoietin-like protein 4: health effects, modulating agents and structure-function relationships. Expert Rev Proteomics, 2012. 9(2): p. 181-99. 
211. Lichtenstein, L., et al., Angptl4 upregulates cholesterol synthesis in liver via inhibition of LPL- and HL-dependent hepatic cholesterol uptake. Arterioscler Thromb Vasc Biol, 2007. 27(11): p. 2420-7.

212. Mandard, S., et al., The fasting-induced adipose factor/angiopoietin-like protein 4 is physically associated with lipoproteins and governs plasma lipid levels and adiposity. J Biol Chem, 2006. 281(2): p. 934-44.

213. Ge, H., et al., Oligomerization state-dependent hyperlipidemic effect of angiopoietinlike protein 4. J Lipid Res, 2004. 45(11): p. 2071-9.

214. Tan, M.J., et al., Emerging roles of angiopoietin-like 4 in human cancer. Mol Cancer Res, 2012. 10(6): p. 677-88.

215. Goh, Y.Y., et al., Angiopoietin-like 4 interacts with matrix proteins to modulate wound healing. J Biol Chem, 2010. 285(43): p. 32999-3009.

216. Yang, Y.H., et al., Suppression of the Raf/MEK/ERK signaling cascade and inhibition of angiogenesis by the carboxyl terminus of angiopoietin-like protein 4. Arterioscler Thromb Vasc Biol, 2008. 28(5): p. 835-40.

217. Kim, H.K., et al., Hypothalamic Angptl4/Fiaf is a novel regulator of food intake and body weight. Diabetes, 2010. 59(11): p. 2772-80.

218. Kobayashi, J., et al., Lipoprotein lipase mass and activity in severe hypertriglyceridemia. Clin Chim Acta, 1993. 216(1-2): p. 113-23.

219. Xu, A., et al., Angiopoietin-like protein 4 decreases blood glucose and improves glucose tolerance but induces hyperlipidemia and hepatic steatosis in mice. Proc Natl Acad Sci U S A, 2005. 102(17): p. 6086-91.

220. Yamada, T., et al., Insulin downregulates angiopoietin-like protein $4 \mathrm{mRNA}$ in 3T3L1 adipocytes. Biochem Biophys Res Commun, 2006. 347(4): p. 1138-44.

221. Oliver, E., et al., The role of inflammation and macrophage accumulation in the development of obesity-induced type 2 diabetes mellitus and the possible therapeutic effects of long-chain n-3 PUFA. Proc Nutr Soc, 2010. 69(2): p. 232-43.

222. Osborn, O. and J.M. Olefsky, The cellular and signaling networks linking the immune system and metabolism in disease. Nat Med, 2012. 18(3): p. 363-74.

223. Hotamisligil, G.S., N.S. Shargill, and B.M. Spiegelman, Adipose expression of tumor necrosis factor-alpha: direct role in obesity-linked insulin resistance. Science, 1993. 259(5091): p. 87-91.

224. Uysal, K.T., et al., Protection from obesity-induced insulin resistance in mice lacking TNF-alpha function. Nature, 1997. 389(6651): p. 610-4.

225. Shoelson, S.E., J. Lee, and A.B. Goldfine, Inflammation and insulin resistance. J Clin Invest, 2006. 116(7): p. 1793-801.

226. Lichtenstein, L., et al., Angptl4 protects against severe proinflammatory effects of saturated fat by inhibiting fatty acid uptake into mesenteric lymph node macrophages. Cell Metab, 2010. 12(6): p. 580-92.

227. Georgiadi, A., et al., Overexpression of angiopoietin-like protein 4 protects against atherosclerosis development. Arterioscler Thromb Vasc Biol, 2013. 33(7): p. 152937. 
228. Hermann, L.M., et al., Angiopoietin-like-4 is a potential angiogenic mediator in arthritis. Clin Immunol, 2005. 115(1): p. 93-101.

229. Li, L., et al., Angiopoietin-like 4 Increases Pulmonary Tissue Leakiness and Damage during Influenza Pneumonia. Cell Rep, 2015.

230. Tjeerdema, N., et al., Inflammation increases plasma angiopoietin-like protein 4 in patients with the metabolic syndrome and type 2 diabetes. BMJ Open Diabetes Res Care, 2014. 2(1): p. e000034.

231. Lu, B., et al., The acute phase response stimulates the expression of angiopoietin like protein 4. Biochem Biophys Res Commun, 2010. 391(4): p. 1737-41.

232. Frenzel, E., et al., Acute-phase protein alphal-antitrypsin--a novel regulator of angiopoietin-like protein 4 transcription and secretion. J Immunol, 2014. 192(11): p. 5354-62.

233. Allavena, P., et al., The chemokine receptor switch paradigm and dendritic cell migration: its significance in tumor tissues. Immunol Rev, 2000. 177: p. 141-9.

234. Torisu, H., et al., Macrophage infiltration correlates with tumor stage and angiogenesis in human malignant melanoma: possible involvement of TNFalpha and IL-1alpha. Int J Cancer, 2000. 85(2): p. 182-8.

235. Schoppmann, S.F., et al., Tumor-associated macrophages express lymphatic endothelial growth factors and are related to peritumoral lymphangiogenesis. Am J Pathol, 2002. 161(3): p. 947-56.

236. Dennis, K.L., et al., Current status of interleukin-10 and regulatory T-cells in cancer. Curr Opin Oncol, 2013. 25(6): p. 637-45.

237. Coussens, L.M. and Z. Werb, Inflammation and cancer. Nature, 2002. 420(6917): p. 860-7.

238. Rakoff-Nahoum, S., Why cancer and inflammation? Yale J Biol Med, 2006. 79(3-4): p. 123-30.

239. Zhu, H., et al., [Cloning of a novel gene, ANGPTL4 and the functional study in angiogenesis]. Zhonghua Yi Xue Za Zhi, 2002. 82(2): p. 94-9.

240. Le Jan, S., et al., Angiopoietin-like 4 is a proangiogenic factor produced during ischemia and in conventional renal cell carcinoma. Am J Pathol, 2003. 162(5): p. 1521-8.

241. Nakayama, T., et al., Expression of angiopoietin-like 4 in human gastric cancer: ANGPTL4 promotes venous invasion. Oncol Rep, 2010. 24(3): p. 599-606.

242. Hu, J., et al., Angiopoietin-like 4: a novel molecular hallmark in oral Kaposi's sarcoma. Oral Oncol, 2011. 47(5): p. 371-5.

243. Dong, D., et al., Serum level of ANGPTL4 as a potential biomarker in renal cell carcinoma. Urol Oncol, 2017.

244. Shibata, K., et al., Clinicopathological significance of angiopoietin-like protein 4 expression in oesophageal squamous cell carcinoma. J Clin Pathol, 2010. 63(12): p. 1054-8.

245. Tanaka, J., et al., ANGPTL4 regulates the metastatic potential of oral squamous cell carcinoma. J Oral Pathol Med, 2015. 44(2): p. 126-33. 
246. De Fazio, L., et al., Longitudinal analysis of inflammation and microbiota dynamics in a model of mild chronic dextran sulfate sodium-induced colitis in mice. World J Gastroenterol, 2014. 20(8): p. 2051-61.

247. Berry, D., et al., Intestinal Microbiota Signatures Associated with Inflammation History in Mice Experiencing Recurring Colitis. Front Microbiol, 2015. 6: p. 1408.

248. Brinkman, B.M., et al., Gut microbiota affects sensitivity to acute DSS-induced colitis independently of host genotype. Inflamm Bowel Dis, 2013. 19(12): p. 2560-7.

249. Wu, F., et al., Genome-wide gene expression differences in Crohn's disease and ulcerative colitis from endoscopic pinch biopsies: insights into distinctive pathogenesis. Inflamm Bowel Dis, 2007. 13(7): p. 807-21.

250. Loddo, I. and C. Romano, Inflammatory Bowel Disease: Genetics, Epigenetics, and Pathogenesis. Front Immunol, 2015. 6: p. 551.

251. Ek, W.E., M. D'Amato, and J. Halfvarson, The history of genetics in inflammatory bowel disease. Ann Gastroenterol, 2014. 27(4): p. 294-303.

252. Borges, H.L., R. Linden, and J.Y. Wang, DNA damage-induced cell death: lessons from the central nervous system. Cell Res, 2008. 18(1): p. 17-26.

253. Liu, D. and Y. Xu, p53, oxidative stress, and aging. Antioxid Redox Signal, 2011. 15(6): p. 1669-78.

254. Omori, E., et al., Ablation of TAK1 upregulates reactive oxygen species and selectively kills tumor cells. Cancer Res, 2010. 70(21): p. 8417-25.

255. Lam, C.R., et al., TAK1 regulates SCF expression to modulate PKBalpha activity that protects keratinocytes from ROS-induced apoptosis. Cell Death Differ, 2011. 18(7): p. 1120-9.

256. Chan, J.S., et al., Cancer-associated fibroblasts enact field cancerization by promoting extratumoral oxidative stress. Cell Death Dis, 2017. 8(1): p. e2562.

257. Slaughter, D.P., H.W. Southwick, and W. Smejkal, Field cancerization in oral stratified squamous epithelium; clinical implications of multicentric origin. Cancer, 1953. 6(5): p. 963-8.

258. Kubo, H., et al., Regulation and clinical significance of the hypoxia-induced expression of ANGPTL4 in gastric cancer. Oncol Lett, 2016. 11(2): p. 1026-1034.

259. Zhu, X., et al., ANGPTL4 Correlates with NSCLC Progression and Regulates Epithelial-Mesenchymal Transition via ERK Pathway. Lung, 2016. 194(4): p. 637-46.

260. Jiang, K., et al., Regulation of Chk1 includes chromatin association and 14-3-3 binding following phosphorylation on Ser-345. J Biol Chem, 2003. 278(27): p. 25207-17.

261. Forrest, A. and B. Gabrielli, Cdc25B activity is regulated by 14-3-3. Oncogene, 2001. 20(32): p. 4393-401.

262. Wang, H., et al., Dysregulation of TTP and HuR plays an important role in cancers. Tumour Biol, 2016. 37(11): p. 14451-14461.

263. Griseri, P. and G. Pages, Control of pro-angiogenic cytokine mRNA half-life in cancer: the role of AU-rich elements and associated proteins. J Interferon Cytokine Res, 2014. 34(4): p. 242-54. 
264. Young, L.E., et al., The $m R N A$ binding proteins HuR and tristetraprolin regulate cyclooxygenase 2 expression during colon carcinogenesis. Gastroenterology, 2009. 136(5): p. 1669-79.

265. Dolan, D.E. and S. Gupta, PD-1 pathway inhibitors: changing the landscape of cancer immunotherapy. Cancer Control, 2014. 21(3): p. 231-7.

266. Wang, X., et al., PD-L1 expression in human cancers and its association with clinical outcomes. Onco Targets Ther, 2016. 9: p. 5023-39.

267. Gandini, S., D. Massi, and M. Mandala, PD-L1 expression in cancer patients receiving anti $P D-1 / P D-L 1$ antibodies: A systematic review and meta-analysis. Crit Rev Oncol Hematol, 2016. 100: p. 88-98.

268. Zhang, L.S. and S.S. Davies, Microbial metabolism of dietary components to bioactive metabolites: opportunities for new therapeutic interventions. Genome Med, 2016. 8(1): p. 46.

269. Corwin, R.L., et al., Dietary saturated fat intake is inversely associated with bone density in humans: analysis of NHANES III. J Nutr, 2006. 136(1): p. 159-65.

270. Martinez-Ramirez, M.J., et al., Dietary fat intake and the risk of osteoporotic fractures in the elderly. Eur J Clin Nutr, 2007. 61(9): p. 1114-20.

271. Johansson, C., et al., Bone mineral density is a predictor of survival. Calcif Tissue Int, 1998. 63(3): p. 190-6.

272. von der Recke, P., M.A. Hansen, and C. Hassager, The association between low bone mass at the menopause and cardiovascular mortality. Am J Med, 1999. 106(3): p. 273-8.

273. Albala, C., et al., Obesity as a protective factor for postmenopausal osteoporosis. Int J Obes Relat Metab Disord, 1996. 20(11): p. 1027-32.

274. Reid, I.R., L.D. Plank, and M.C. Evans, Fat mass is an important determinant of whole body bone density in premenopausal women but not in men. J Clin Endocrinol Metab, 1992. 75(3): p. 779-82.

275. Gomez-Ambrosi, J., et al., The bone-adipose axis in obesity and weight loss. Obes Surg, 2008. 18(9): p. 1134-43.

276. Fukumoto, S. and T.J. Martin, Bone as an endocrine organ. Trends Endocrinol Metab, 2009. 20(5): p. 230-6.

277. Shafik, N.M., et al., Significance of Tissue Expression and Serum Levels of Angiopoietin-like Protein 4 in Breast Cancer Progression: Link to NF-kappaB /P65 Activity and Pro-Inflammatory Cytokines. Asian Pac J Cancer Prev, 2015. 16(18): p. 8579-87.

278. Condeelis, J. and J.W. Pollard, Macrophages: obligate partners for tumor cell migration, invasion, and metastasis. Cell, 2006. 124(2): p. 263-6.

279. Noy, R. and J.W. Pollard, Tumor-associated macrophages: from mechanisms to therapy. Immunity, 2014. 41(1): p. 49-61.

280. Sica, A. and A. Mantovani, Macrophage plasticity and polarization: in vivo veritas. $\mathrm{J}$ Clin Invest, 2012. 122(3): p. 787-95. 
281. Mantovani, A., et al., Macrophage plasticity and polarization in tissue repair and remodelling. J Pathol, 2013. 229(2): p. 176-85.

282. Liu, C.Y., et al., M2-polarized tumor-associated macrophages promoted epithelialmesenchymal transition in pancreatic cancer cells, partially through TLR4/IL-10 signaling pathway. Lab Invest, 2013. 93(7): p. 844-54.

283. Yamaguchi, T., et al., Tumor-associated macrophages of the M2 phenotype contribute to progression in gastric cancer with peritoneal dissemination. Gastric Cancer, 2016. 19(4): p. 1052-65.

284. Schumann, T., et al., Deregulation of PPARbeta/delta target genes in tumorassociated macrophages by fatty acid ligands in the ovarian cancer microenvironment. Oncotarget, 2015. 6(15): p. 13416-33.

285. Schmid, M., et al., Association of elevated $C$-reactive protein levels with an impaired prognosis in patients with surgically treated endometrial cancer. Obstet Gynecol, 2007. 110(6): p. 1231-6.

286. Wu, Y.Q., et al., Serum angiopoietin-like 4 is over-expressed in COPD patients: association with pulmonary function and inflammation. Eur Rev Med Pharmacol Sci, 2016. 20(1): p. 44-53.

287. Waschki, B., et al., Angiopoietin-like protein 4 and cardiovascular function in COPD. BMJ Open Respir Res, 2016. 3(1): p. e000161.

288. Hersoug, L.G., P. Moller, and S. Loft, Gut microbiota-derived lipopolysaccharide uptake and trafficking to adipose tissue: implications for inflammation and obesity. Obes Rev, 2016. 17(4): p. 297-312. 


\section{Angiopoietin-like 4 Mediates Colonic Inflammation by Regulating Chemokine Transcript Stability via Tristetraprolin}

Terri PHUA, Ming Keat SNG, Eddie Han Pin TAN, Dickson Shao Liang CHEE, Yinliang LI, Jonathan Wei Kiat WEE, Ziqiang TEO, Jeremy Soon Kiat CHAN, Maegan Miang Kee LIM, Chek Kun TAN, Pengcheng ZHU, Velmurugesan ARULAMPALAM, Nguan Soon TAN*

*Corresponding Author.

Scientific Reports. 2017 Mar 13;7:44351. doi: 10.1038/srep44351. 


\section{SCIENTIFIC REPRTS}

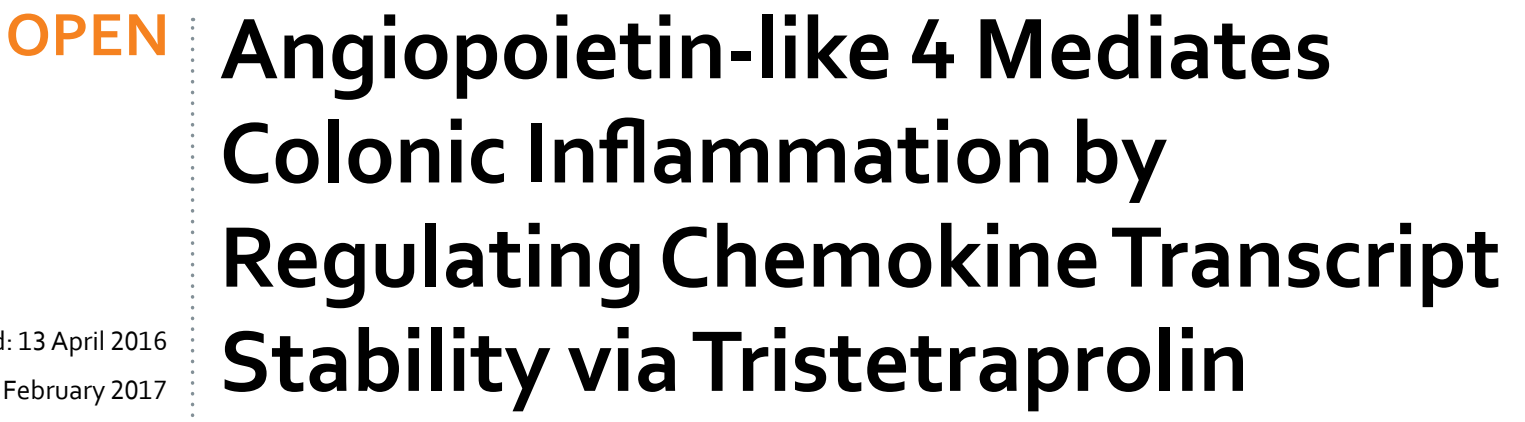

Accepted: 09 February 2017

Published: 13 March 2017

Terri Phua ${ }^{1,2}$, Ming Keat Sng ${ }^{1,3}$, Eddie Han Pin Tan ${ }^{1}$, Dickson Shao Liang Chee ${ }^{1}$, Yinliang $\mathrm{Li}^{1}$, Jonathan Wei Kiat Wee ${ }^{1}$, Ziqiang Teo ${ }^{1}$, Jeremy Soon Kiat Chan ${ }^{1}$, Maegan Miang Kee Lim ${ }^{1}$, Chek Kun Tan ${ }^{3}$, Pengcheng Zhu ${ }^{1}$, Velmurugesan Arulampalam ${ }^{2}$ \& Nguan Soon Tan ${ }^{1,3,4,5}$

Many gastrointestinal diseases exhibit a protracted and aggravated inflammatory response that can lead to hypercytokinaemia, culminating in extensive tissue damage. Recently, angiopoietin-like 4 (ANGPTL4) has been implicated in many inflammation-associated diseases. However, how ANGPTL4 regulates colonic inflammation remains unclear. Herein, we show that ANGPTL4 deficiency in mice (ANGPTL4 ${ }^{-l-}$ ) exacerbated colonic inflammation induced by dextran sulfate sodium (DSS) or stearic acid. Microbiota was similar between the two genotypes prior DSS challenge. A microarray gene expression profile of the colon from DSS-treated ANGPTL4 ${ }^{-l-}$ mice was enriched for genes involved in leukocyte migration and infiltration, and showed a close association to inflamed ulcerative colitis (UC), whereas the profile from ANGPTL4 ${ }^{+++}$littermates resembled that of non-inflamed UC biopsies. Bone marrow transplantation demonstrates the intrinsic role of colonic ANGPTL4 in regulating leukocyte infiltration during DSS-induced inflammation. Using immortalized human colon epithelial cells, we revealed that the ANGPTL4-mediated upregulation of tristetraprolin expression operates through CREB and NF- $K B$ transcription factors, which in turn, regulates the stability of chemokines. Together, our findings suggest that ANGPTL4 protects against acute colonic inflammation and that its absence exacerbates the severity of inflammation. Our findings emphasize the importance of ANGPTL4 as a novel target for therapy in regulating and attenuating inflammation.

An aggravated inflammatory response is a common feature of many gastrointestinal disorders, such as inflammatory bowel diseases, enteritis, and colitis. Many of these conditions are caused by changes in dietary fat intake, the ingestion of bacteria-contaminated food and water, and certain chemicals. These insults trigger an inflammatory response by inducing the recruitment of macrophages to the site of inflammation to combat pathogens, neutralize harmful immunogens and promote tissue repair ${ }^{1}$. However, a protracted inflammatory response can cause tissue damage and lead to hypercytokinaemia, which is a potentially fatal immune reaction. Immune cell infiltration into the site of damage is highly regulated by chemotactic factors, such as macrophage inflammatory protein $1 \alpha$ and chemokine (C-C motif) ligand 2 (CCL2) $)^{2,3}$. As the initial cellular barrier that encounters lumenal insults, intestinal and colonic epithelia play important roles in the early recruitment of inflammatory cells to the mucosa. Epithelial cells are a major source of chemoattractants, and epithelial chemokine production has been proposed as a key target of future therapies for gastrointestinal disorders ${ }^{4}$. However, much remains to be understood about the mechanisms that regulate the levels of these chemokines in the gastrointestinal and colonic tracts.

\footnotetext{
${ }^{1}$ School of Biological Sciences, Nanyang Technological University, 60 Nanyang Drive, Singapore 637551, Singapore. 2Department of Microbiology, Tumor and Cell Biology, Karolinska Institutet, Nobels väg 16, Stockholm 17177, Sweden. ${ }^{3}$ Lee Kong Chian School of Medicine, Nanyang Technological University, 50 Nanyang Drive, Singapore 639798, Singapore. ${ }^{4}$ Institute of Molecular Cell Biology, 61 Biopolis Drive, Proteos, Agency for Science Technology \& Research, Singapore 138673, Singapore. ${ }^{5}$ KK Research Centre, KK Women's and Children Hospital, 100 Bukit Timah Road, Singapore 229899, Singapore. Correspondence and requests for materials should be addressed to N.S.T. (email: nstan@ntu.edu.sg)
} 
Angiopoietin-like 4 (ANGPTL4) is a matricellular protein that has been implicated in many inflammation-associated diseases ${ }^{5}$. Native full-length ANGPTL4 (fANGPTL4) is proteolytically cleaved into two functionally distinct isoforms: the N-terminal domain (nANGPTL4) inhibits lipoprotein lipase (LPL) and directly regulates energy homeostasis, while the C-terminal domain (cANGPTL4) has been implicated in various processes such as cancer metastasis, skin wound and pulmonary inflammation ${ }^{6-8}$. Diabetic wounds show low endogenous cANGPTL4 levels and have been associated with an elevated $\mathrm{F} 4 / 80^{+}$macrophage population at the wound site. The infiltration of $\mathrm{F} 4 / 80^{+}$macrophages was reduced upon treatment of diabetic wounds with recombinant cANGPTL4 when compared with saline ${ }^{9}$. ANGPTL4 can also protect against the severe pro-inflammatory effects of saturated fat by inhibiting fatty acid uptake by mesenteric lymph node macrophages ${ }^{10}$. Similarly, ANGPTL4 confers protective effects against the development of atherosclerosis ${ }^{11}$, which has been associated with atherogenesis and macrophage polarization ${ }^{12}$. ANGPTL4 has also been identified as an angiogenic mediator in arthritis ${ }^{13}$. ANGPTL4 has been observed to exacerbate influenza-associated inflammation through IL-6-Stat3 signaling in the lung ${ }^{14}$. Furthermore, serum ANGPTL4 was associated with the C-reactive protein level in type II diabetic patients, suggesting that ANGPTL4 may be involved in the progression of inflammation during metabolic syndrome ${ }^{15}$. Thus, ANGPTL4 may exert both anti- and pro-inflammatory effects in a context-dependent manner. Despite numerous reports of the role of ANGPTL4 in inflammation, the mechanisms whereby ANGPTL4 modulates inflammation in various diseases remain largely unclear.

Herein, we describe an anti-inflammatory role for colonic ANGPTL4 in dextran sulfate sodium salt (DSS)-induced colitis and dietary stearic acid (SA) intake in vitro and in vivo. We showed that the microbiota was similar between ANGPTL4 ${ }^{+/+}$and ANGPTL4 $4^{-1-}$ mice at steady states, but with perturbation such as DSS treatment some differences in microbiota community become accentuated. Bone marrow transplantation and microarray analysis confirmed the intrinsic role of colonic ANGPTL4 in regulating leukocyte infiltration during DSS-induced inflammation, and thus the colonic inflammatory landscape. The underlying mechanism involves the regulation of tristetraprolin (TTP or ZFP36), an mRNA-binding protein that is involved in chemokine destabilization, by ANGPTL4 via activation of CREB and NF- $\kappa B$ transcription factors.

\section{Results}

ANGPTL4 reduces DSS- and saturated fat-induced colonic inflammation. We first characterized the intestinal and colonic tract of unchallenged ANGPTL4-knockout (ANGPTL4 ${ }^{-1-}$ ) and wild-type (ANGPTL4 ${ }^{+/+}$) mice. There was no significant difference in body weight, colon length, disease activity index (DAI), endpoint macroscopic scores or histological scores between the genotypes (Fig. 1a and Supplementary Fig. S1a,c). Detailed examination revealed that ANGPTL4 ${ }^{-1-}$ mice exhibited an increased muscularis thickness and shorter colonic villus length than ANGPTL4 ${ }^{+/+}$littermates (Fig. 1b, Supplementary Fig. S1d,e). To gain insights into the role of ANGPTL4 in acute colonic inflammation, we challenged ANGPTL4 ${ }^{-1-}$ and ANGPTL $4^{+I+}$ mice to either $5 \%$ DSS or $15 \%(w / w)$ stearic acid (SA) for 8 days.

ANGPTL4 ${ }^{-1-}$ mice receiving DSS lost significantly more weight compared with ANGPTL $4^{+/+}$mice from day 6 onwards (Supplementary Fig. S1a). DSS-treatment resulted in a significant increase in both the DAI and endpoint macroscopic score (Fig. $1 \mathrm{c}$ and Supplementary Fig. S1b) as well as a reduction in the ANGPTL4 ${ }^{+/+}$colonic villus length, whereas there was no significant reduction observed in ANGPTL4 ${ }^{-1-}$ mice compared with their cognate controls (Fig. $1 \mathrm{~b}$ and Supplementary Fig. S1e). Histological analysis of the DSS-challenged ANGPTL4 ${ }^{-/-}$ colon sections revealed a severe loss of epithelial integrity and a massive infiltration of immune cells into the lamina propria when compared with cognate ANGPTL4 ${ }^{+/+}$colon (Fig. 1b). Indeed, we observed a higher number of Ly6G ${ }^{+}$neutrophils and $\mathrm{CD}^{+} 8^{+}$macrophages in the colons of DSS-treated ANGPTL4 ${ }^{-1-}$ mice (Fig. $1 \mathrm{~d}$ and Supplementary Fig. S1f). FACS analysis also confirmed the higher number of Ly6G ${ }^{+}, \mathrm{CD}_{11 \mathrm{~b}^{+}}$and F4/80 $0^{+}$cells in ANGPTL4 ${ }^{-1-}$ mice compared to ANGPTL4 ${ }^{+/+}$mice (Fig. 1e,f). Such differences were not attributed to an increase in apoptotic cells between the genotypes, as indicated by TUNEL-positive cells and the expression of cleaved caspase 3 (Supplementary Fig. S1g). Consistent with the elevated inflammation status, we also detected higher levels of TNF- $\alpha$, IL-6 and IFN- $\gamma$ mRNA transcripts (Fig. 1g).

As expected, stearic acid induced a more subdued inflammatory response compared to the DSS challenge (Fig. 1g). No significant differences in weight gain, blood glucose levels, DAI, endpoint macroscopic scores or histological scores were observed between ANGPTL4 ${ }^{-1-}$ and ANGPTL4 ${ }^{+/+}$mice on the SA diet compared with mice fed a normal chow diet (Fig. $1 \mathrm{c}$ and Supplementary Fig. S1a-c,h). ANGPTL4 ${ }^{+/+}$mice on an SA diet exhibited a longer colon, a thicker muscularis wall and a shorter colonic villus length at the endpoint compared with mice fed a standard diet (Fig. 1a,b and Supplementary Fig. S1c,d). Immunofluorescence staining showed a similar elevated number of $\mathrm{Ly}_{6 \mathrm{G}}{ }^{+}$neutrophils and $\mathrm{CD}^{+} 8^{+}$macrophages in ANGPTL4 ${ }^{-1-}$ colon (Fig. $1 \mathrm{~d}$ and Supplementary Fig. S1f). FACS analysis also detected an elevated number of Ly6G ${ }^{+}, \mathrm{CD}_{11 \mathrm{~b}}{ }^{+}$and F4/80 $0^{+}$cells in ANGPTL4 $^{-l-}$ mice (Fig. 1e,f). Together, our data demonstrated that the absence of ANGPTL4 enriched for a subset of the immune cell population not limited to neutrophils, macrophages and eosinophils during inflammation. Altogether, our observations are consistent with a model whereby ANGPTL4 attenuates colonic inflammation in response to inflammatory stimuli.

ANGPTL4 deficiency does not influence colonic commensal microbiota at steady state. Several reports have suggested that dysbiosis aggravates host inflammatory and metabolic diseases ${ }^{16-19}$. Studies also suggested that a subset of microbes could regulate ANGPTL4 expression in the gut in response to changes in energy demand $\mathrm{s}^{20-22}$. To examine if the exacerbated colonic inflammatory response in ANGPTL4 ${ }^{-1-}$ mice could be attributed to microbe composition, we performed $16 \mathrm{~S}$ metagenomics sequencing using the V4 region to identify microbe abundance between ANGPTL4 $4^{+/+}$and ANGPTL4 ${ }^{-/-}$littermates before and after DSS-induced inflammation. Redundancy analysis showed that genotype and inflammation amounted to $41.2 \%$ of the total variation observed between microbial compositions in the colon. Genotype could only explain for $10.7 \%$ of the differences 
a

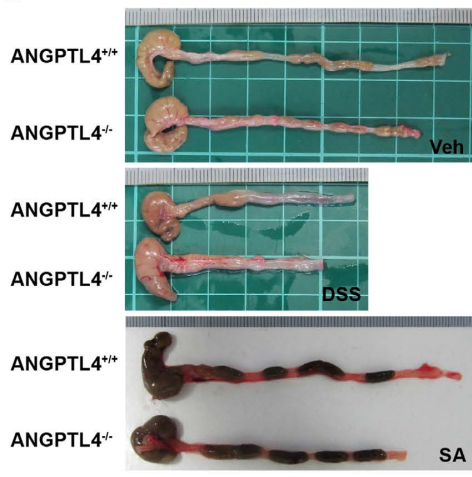

C

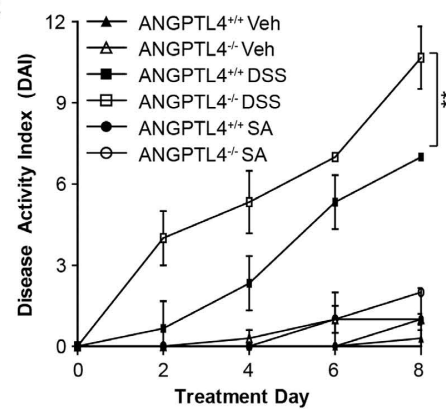

d

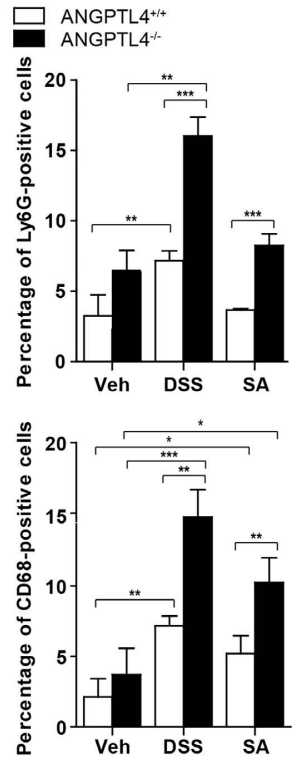

b

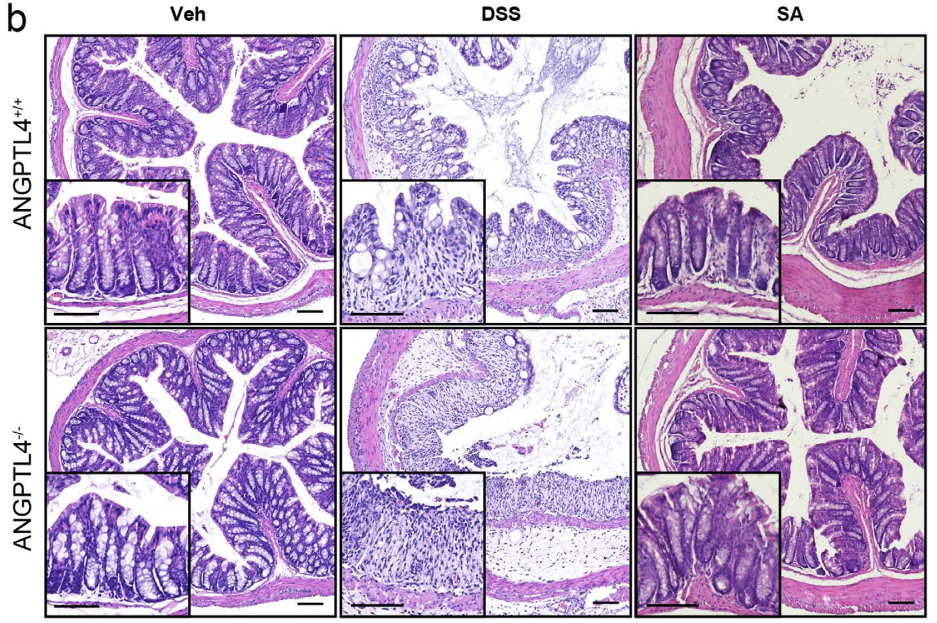

e

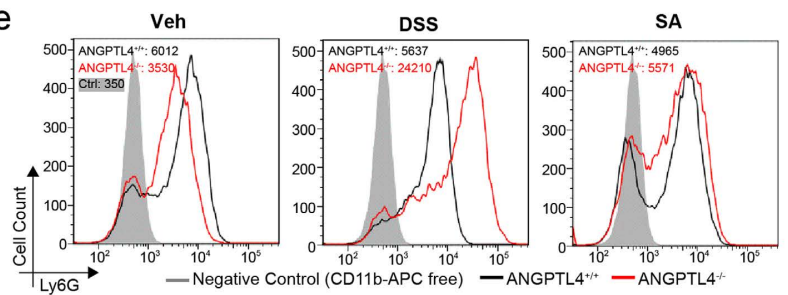

f
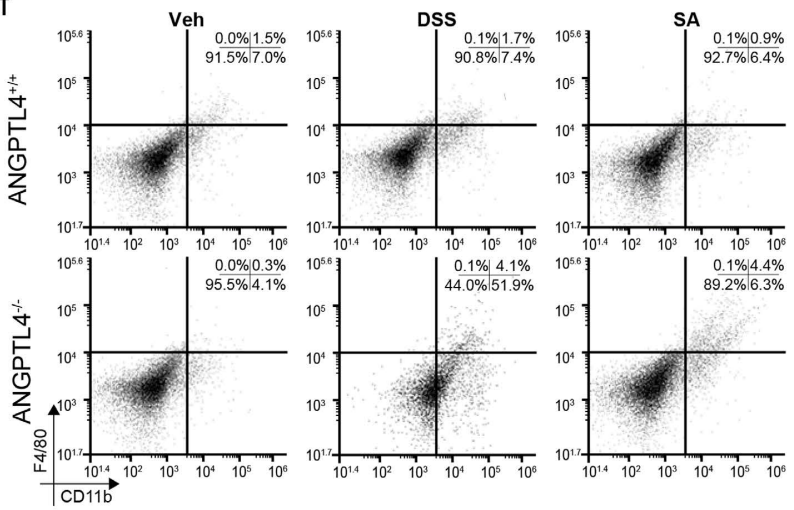

g

$\square$ ANGPTL4 $^{+/+}$Veh $⿴$ ANGPTL4 ${ }^{+/+}$SA $\square \square$ ANGPTL4 ${ }^{+/+}$DSS

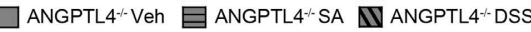

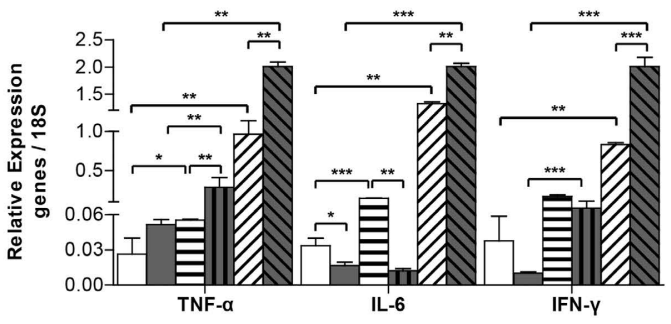

Figure 1. ANGPTL4 attenuates colonic inflammation. (a-e) Images of colon samples (a) Representative colon sections stained with hematoxylin and eosin (H\&E) (b). (c) Disease activity index (DAI) of mice assessed every alternate day over the treatment regimes. Scoring criteria can be found in Supplementary Table S1. (d) Percentage of Ly6G $\mathrm{G}^{+}$and $\mathrm{CD} 68^{+}$cells per field of view. Total DAPI-stained nuclei were taken as the total cell number. Microscopic views from 5 different sections were numerated. (e) Representative FACS histograms for Ly6G expression (neutrophils) from ANGPTL4 ${ }^{+/+}$and ANGPTL4 $^{-/-}$littermates for the indicated treatments. (f) Representative FACS analysis of the colons from ANGPTL4 ${ }^{+/+}$and ANGPTL4 ${ }^{-1-}$ mice double stained for F4/80 (macrophages) and CD11b (macrophages, monocytes, granulocytes, NK cells, dendritic cells) for each of the treatments. (g) Relative mRNA levels of pro-inflammatory cytokines (TNF- $\alpha$, IL- 6 and IFN- $\gamma$ ) from ANGPTL $^{+/+}$and ANGPTL4 ${ }^{-1-}$ mice at the endpoint. For the Veh, DSS and SA groups, $\mathrm{n}=20$ mice were used for each treatment. The Mann-Whitney $U$ test was used. ${ }^{*} \mathrm{p}<0.05 ;{ }^{* *} \mathrm{p}<0.01$. 

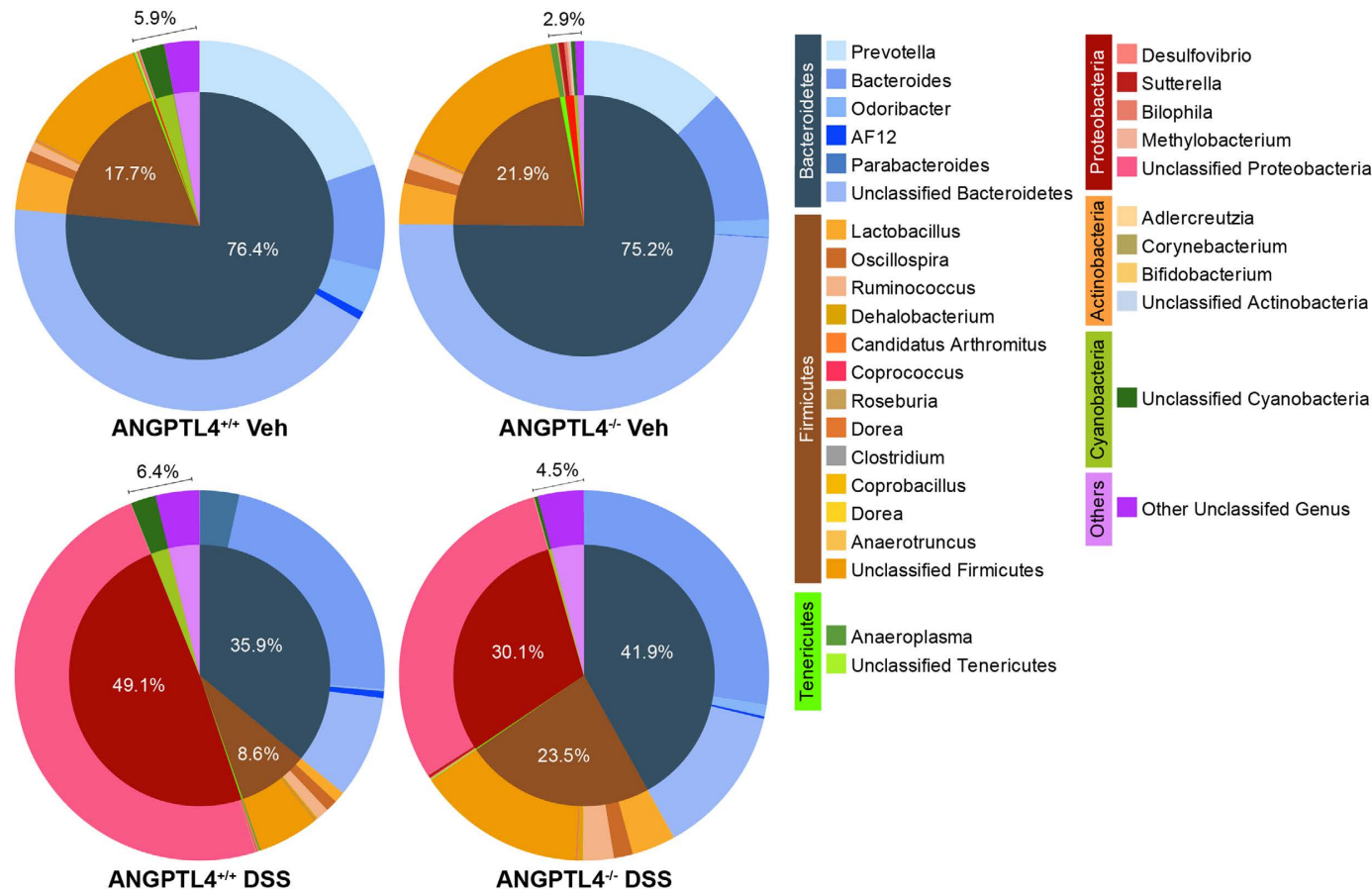

Figure 2. ANGPTL4 deficiency does not influence commensal microbe composition. Pie charts showing the commensal microbe landscape at steady state (upper panel) and after DSS treatment (lower panel) of age- and gender- matched ANGPTL4 ${ }^{+/+}$and ANGPTL $4^{-/-}$littermates. Values in the charts denote mean percentage of abundance. $\mathrm{n}=6$ mice per group.

observed, while inflammation amounted to at least $31.1 \%$. The steady state phylogenetic microbe composition was predominated by Firmicutes and Bacteriodetes, which did not differ much between both ANGPTL4 ${ }^{+/+}$ and ANGPTL4 ${ }^{-1-}$ mice before DSS treatment (Fig. 2). As expected, DSS-induced inflammation caused a sharp decrease in Bacteroidetes and a concomitant increase in Firmicutes and Proteobacteria. We found that Firmicutes were more abundant in inflamed ANGPTL4 ${ }^{+/+}$mice, while Proteobacteria was more prevalent in ANGPTL4 ${ }^{-/-}$ mice (Fig. 2). Collectively, our data showed that microbe composition was similar between ANGPTL4 ${ }^{+/+}$and ANGPTL4 ${ }^{-1-}$ mice at steady state, but with perturbation such as DSS treatment, some differences in the microbiota community become accentuated.

Intrinsic role of colonic ANGPTL4 in leukocytes infiltration during DSS-induced inflammation. To further define the role of epithelial-derived ANGPTL4, we performed bone marrow transplantation (BMT) experiment. We transferred bone marrow from ANGPTL4 ${ }^{+/+}$and ANGPTL4 ${ }^{-1-}$ donors to $\gamma$-irradiated wild-type $\mathrm{ANGPTL}^{+/+}$(WT IR) recipient mice $\left(\mathrm{BMT}\left(\mathrm{ANGPTL} 4^{+/+}\right)\right.$or BMT $\left(\mathrm{ANGPTL} 4^{-1-}\right)$; Fig. 3a,b). CD4 ${ }^{+}$and $\mathrm{CD}^{+}$cells were depleted from bone marrow inoculum before BMT to limit possible graft-versus-host-disease (GVHD)-related mortality. The bone marrow of WT IR mice appeared necrotic with a significant reduction in the number of bone marrow cells. The spleen was smaller in size and has reduced CD $45^{+}$splenic cells (Fig. 3c,d). Hematopoietic reconstitution was observed through the repopulation of bone marrow cells in the femur and CD $45^{+}$cells in the spleen at 4-week post BMT (Fig. 3c,d). Genotype PCR confirmed the successful reconstitution of the respective donor cells in WT IR mice (Fig. 3e). No significant difference in classical clinical scorings was observed between BMT $\left(\right.$ ANGPTL4 ${ }^{+/+}$) or BMT $\left(\right.$ANGPTL4 $4^{-l-}$ ) chimeras on vehicle treatment (Fig. 3f). Histological analysis also revealed little difference between BMT $\left(\right.$ ANGPTL4 $^{+/+}$) or BMT (ANGPTL4 ${ }^{-/-}$) chi- $^{-}$ meras (Fig. 3g). As expected, DSS-induced inflammation caused an erosion of the epithelial lining with increased infiltration of immune cells into the lamina propria (Fig. $3 \mathrm{~g}$ ). Notably, there was no significant difference recorded in the DAI, endpoint macroscopic score or histological score between BMT $\left(\right.$ ANGPTL4 $^{+/+}$) and BMT $\left(\right.$ ANGPTL4 ${ }^{-l-}$ ) chimeras after DSS treatment (Fig. 3f,g and Supplementary Tables S1-3). FACS analysis also showed no significant difference in the number of $\mathrm{CD} 11 \mathrm{~b}^{+}$and $\mathrm{F} 4 / 80^{+}$cells in the inflamed colons of BMT $\left(\right.$ ANGPTL4 $^{+/+}$) and BMT (ANGPTL4 ${ }^{-l-}$ ) chimeras (Fig. 3h). Taken together, our data demonstrates the intrinsic role of epithelial-derived ANGPTL4 in regulating colonic leukocyte infiltration during DSS-induced inflammation, and thus the colonic inflammatory landscape.

ANGPTL4 deficiency alters colonic inflammatory gene expression. Our observations suggest that ANGPTL4 deficiency increased the susceptibility to inflammation induced by SA and DSS, albeit with differential severities. To strengthen our findings, we performed a comparative microarray gene expression analysis using colon tissues from ANGPTL4 ${ }^{-/-}$and ANGPTL4 $4^{+/+}$mice challenged with either DSS or SA. Using Ingenuity Pathway Analysis (IPA), we identified 250 differentially expressed genes associated with "gastrointestinal diseases". As expected, the gene expression profiles of DSS-challenged colon samples showed a more aggravated 
a

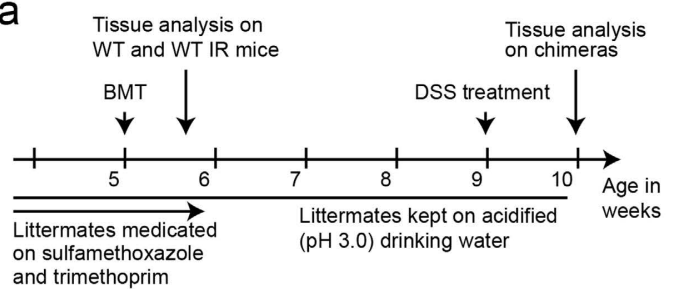

b

Bone marrow donor

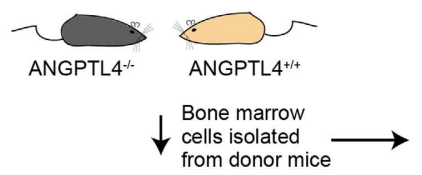

Bone marrow recipient

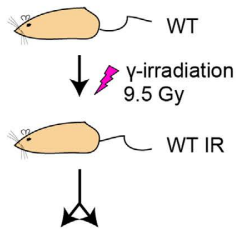

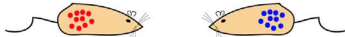
BMT (ANGPTL4 ${ }^{-+}$) BMT (ANGPTL4 ${ }^{+++}$)

C
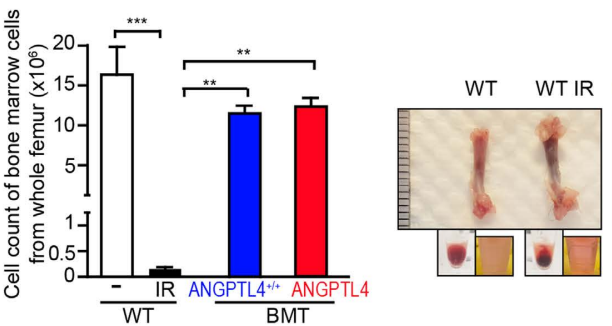

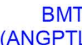

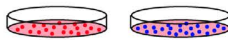
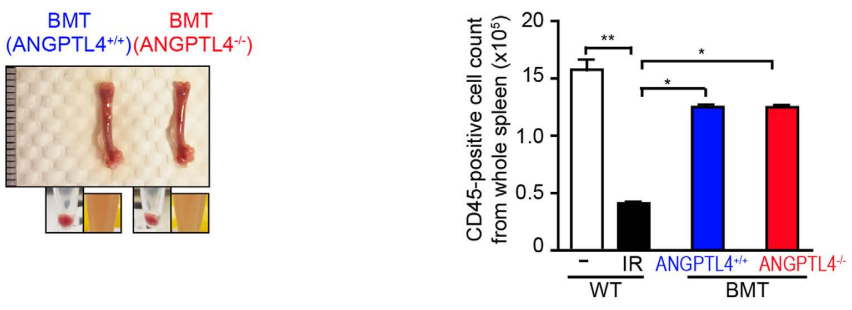

d

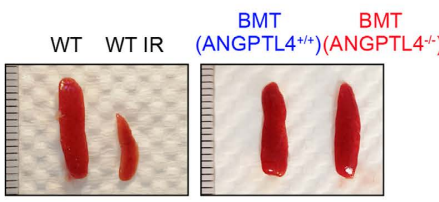

e

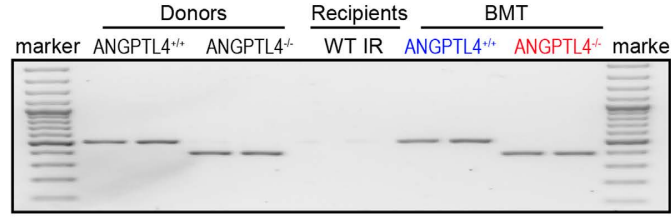

f
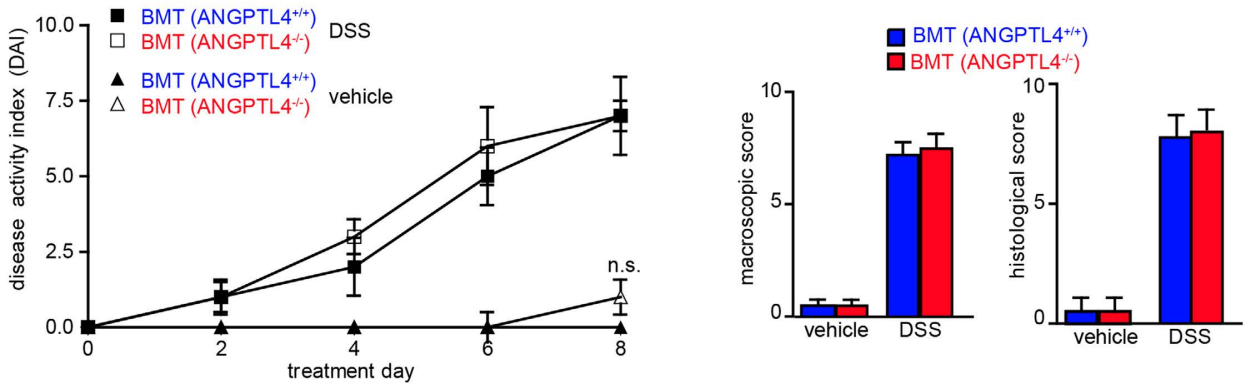

g Vehicle DSS

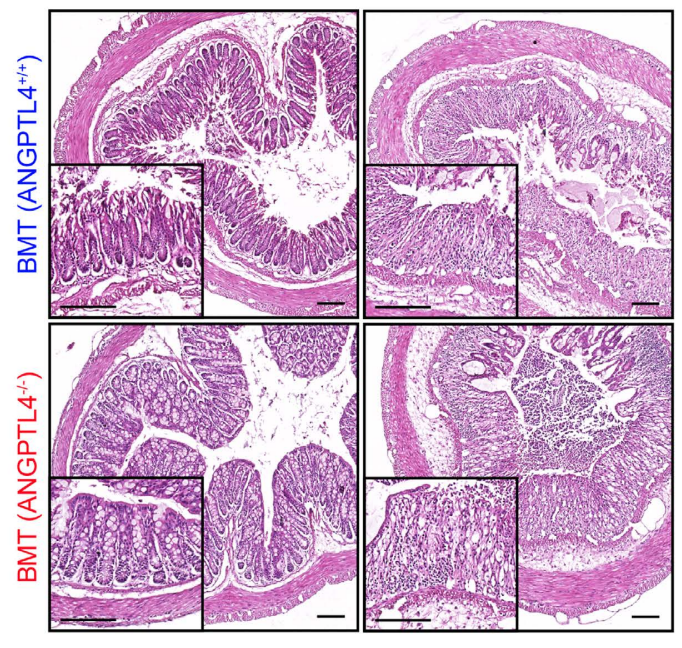

h
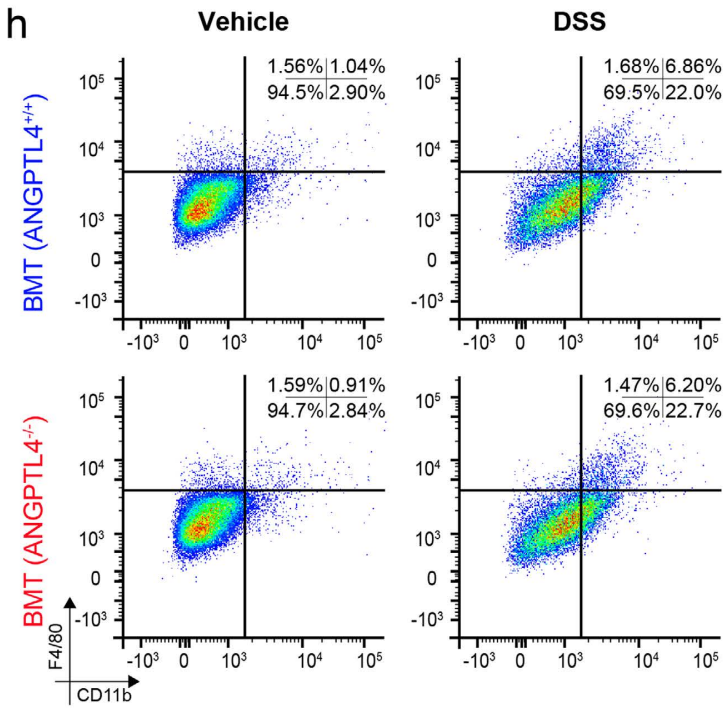

Figure 3. Intrinsic role of colonic ANGPTL4 in leukocytes infiltration during DSS. (a,b) Graphical illustrations depicting the experimental setup for bone marrow transplantation (BMT). CD4+ and CD8+ depleted bone marrow cells from age- and gender-matched ANGPTL $4^{+/+}$or ANGPTL $4^{-/-}$donors were transplanted into $\gamma$-irradiated ANGPTL $^{+/+}$wild-type recipient (WT IR). Chimera BMT ANGPTL4 $4^{+/+}$and BMT ANGPTL4 ${ }^{-1-}$ denote WT IR transplanted with bone marrow cells from ANGPTL4 $4^{+/+}$and ANGPTL4 ${ }^{-/-}$donor, respectively. Graph showed the number of $\mathrm{CD}_{4} 5^{+}$cells from spleen of indicated mice (Mean \pm S.D.; $\mathrm{n}=6$ mice per group). (c,d) Representative 
images of the femur (c) and spleen (d) from indicated mice (WT and WT IR) and chimeras (BMT ANGPTL4 ${ }^{+/+}$ and BMT ANGPTL4 ${ }^{-l-}$ ). Inserts in (c) showed the bone marrow cell pellet before and after red blood cell lysis. Graph in (c; left panel) showed the number of bone marrow cells from femur of indicated mice (Mean \pm S.D.; $\mathrm{n}=6$ mice per group). (e) Representative agarose gel image of genotype PCR products from CD $45^{+}$splenic cells from indicated mice. (f) Graphs showing the disease activity index (DAI; left panel), macroscopic score (middle panel) and histological score (right panel) of indicated mice treated with vehicle or $2 \%$ DSS. Individual scoring criteria can be found in Supplementary Tables S1-S3. (g) Representative hematoxylin and eosin (H\&E) images of colon section from vehicle and DSS treated mice post BMT. (h) Representative FACS analysis for CD11 $\mathrm{b}^{+}$and F4/80+ cells from colons of BMT ANGPTL4 $4^{+/+}$and BMT ANGPTL4 ${ }^{-/-}$chimeras treated with vehicle or DSS. $\mathrm{n}=6$ per group. The MannWhitney $U$ test was used. ${ }^{\star} \mathrm{p}<0.05 ;{ }^{* *} \mathrm{p}<0.01$ and ${ }^{\star * *} \mathrm{p}<0.001$. n.s. denotes not significant.

response when compared with the SA-challenged colon samples. Consistent with the above observations, the heat map also indicated that ANGPTL4 deficiency resulted in more severe inflammation in ANGPTL4 ${ }^{-1-}$ mice when compared with ANGPTL4 ${ }^{+/+}$mice (Fig. 4a,b and Supplementary Table S4). Further studies of the 2 islands of dissimilarly regulated genes between either challenge groups or genotypes revealed that $52 \%$ of the transcripts were related to TNF- $\alpha$ signaling, whereas $27 \%$ and $31 \%$ were linked to the IL-10 and IL- 6 signaling cascades, respectively (Fig. 4c). Gene ontology maps suggested that these genes were primarily involved in leukocyte migration and infiltration (Fig. 4d). The top diseases identified from previous studies listed in the IPA database include colitis, inflammation of the intestine, diabetes mellitus and gastrointestinal tract cancers and tumors (Fig. 4d). Next, a comparative gene expression analysis was performed between DSS-challenged ANGPTL4 ${ }^{-1-}$ and ANGPTL4 ${ }^{+/+}$mice and human ulcerative colitis (UC) colonoscopy samples. The colonic mucosal samples from UC patient biopsies were classified as "inflamed" or "non-inflamed", according to whether the samples exhibited signs of inflammation during collection. Unsupervised hierarchical clustering revealed that the gene expression profile of DSS-challenged ANGPTL $4^{+/+}$mice was more closely associated with the non-inflamed UC samples, while the profile of ANGPTL4 ${ }^{-1-}$ mice was clustered more closely with the inflamed UC samples (Fig. 4e and Supplementary Table S5). Further analysis revealed that the UC samples showed the highest number of up-regulated genes, followed by the DSS-challenged ANGPTL4 ${ }^{-/-}$mice; the DSS-challenged ANGPTL4 ${ }^{+/+}$ mice showed the lowest number of up-regulated genes (Fig. 4f). Our analysis also identified a cluster of distinctively regulated genes across both the mouse and human samples. Among these genes, $29 \%, 27 \%$, and $23 \%$, were involved in the IL-4, IL-1 $\beta$ and IL-6 signaling cascades, respectively (Fig. 4g). Gene ontology analysis revealed that the top molecular and cellular functions included cellular movement, cellular development, cellular function and maintenance, and cellular proliferation, as well as cell death and survival (Fig. 4h). These transcripts have also been found to be involved in myeloid cell homeostasis, immune responses, and leukocyte homeostasis, activation and migration (Fig. 4h). Together, the histological analysis and gene expression profiling indicated that ANGPTL $4^{-1-}$ mice were more susceptible to colonic inflammation, implicating a role for ANGPTL4 in immune cell infiltration.

ANGPTL4 deficiency increases the infiltration of immune cells. ANGPTL4 deficiency exacerbated colonic inflammation upon DSS and SA challenge. Guided by histological differences and gene expression changes between colon samples from ANGPTL4 ${ }^{+/+}$and ANGPTL4 ${ }^{-/-}$mice, we next investigated the chemokine expression profile during inflammation. Consistent with the microarray findings, we detected an overall elevated expression of pro-inflammatory chemokines and a reduced expression of anti-inflammatory cytokines IL-10 and IL-17 in ANGPTL4 ${ }^{-1-}$ mice compared with their ANGPTL4 ${ }^{+/+}$littermates (Fig. 5a). The highest levels of these chemokines were also consistent with greater infiltration of inflammatory cells into the colons of ANGPTL4 ${ }^{-1-}$ mice (Fig. 1d-f and Supplementary Fig. 1f). The protein concentrations of CCL2, CCL11 and CXCL10 were also found to be elevated in ANGPTL4 ${ }^{-1-}$ littermates in both the DSS- and SA- treated groups (Supplementary Fig. S2a). Furthermore, we observe a decreasing trend, albeit not statistically significant, in the protein levels of IL-1 $\beta$, IL-10 and IL-17 between ANGPTL4 ${ }^{+/+}$and ANGPTL4 ${ }^{-/-}$littermates among different treatments.

To date, little is known about the mechanisms by which ANGPTL4 exerts such effects on the immune and chemokine landscape. Recent publications suggest that ANGPTL4 may modulate the inflammatory response in a variety of disease models ${ }^{9-11,14,15,23}$. Our microarray analysis and a review of the literature directed us to two potential intermediary proteins that respond quickly during acute inflammation and acts broadly to attenuate inflammation ${ }^{24,25}$. Human antigen R (HuR) and tristetraprolin (TTP) are mRNA binding proteins well-characterized for their opposite roles in binding mRNA at AU-rich regions located at the $3^{\prime}$-UTR of target transcripts, including chemokines, to modify the inflammatory landscape at the onset of inflammation. HuR is highly selective in its interaction with its cognate mRNA binding partners and has been described as an active participant in promoting mRNA stability ${ }^{26,27}$. In contrast, TTP promotes mRNA degradation by destabilizing target transcripts ${ }^{28-30}$. The acute inflammation elicited by DSS and SA treatments significantly suppressed both ANGPTL4 and TTP expression in ANGPTL4 ${ }^{+/+}$littermates (Supplementary Fig. 2b). However, we detected no significant change in HuR expression in both pro-inflammatory treatments. Analysis of our focused gene expression array revealed that the expression of TTP was altered, as was the expression of genes involved in immune cell infiltration and responses. The reduced mRNA and protein levels of TTP in ANGPTL4 ${ }^{+/+}$and ANGPTL4 ${ }^{-1-}$ littermates were confirmed by real-time PCR and western blot analyses (Supplementary Fig. 2c). Together, our data suggests the involvement of TTP in the regulation of local colonic inflammation.

To further understand the role that colonic epithelial cells play in regulating chemokine expression, we treated human colon epithelial cells (iCECs) with a panel of pro-inflammatory (DSS, SA, IL-1 $\beta$ or TNF- $\alpha$ ) or 
a

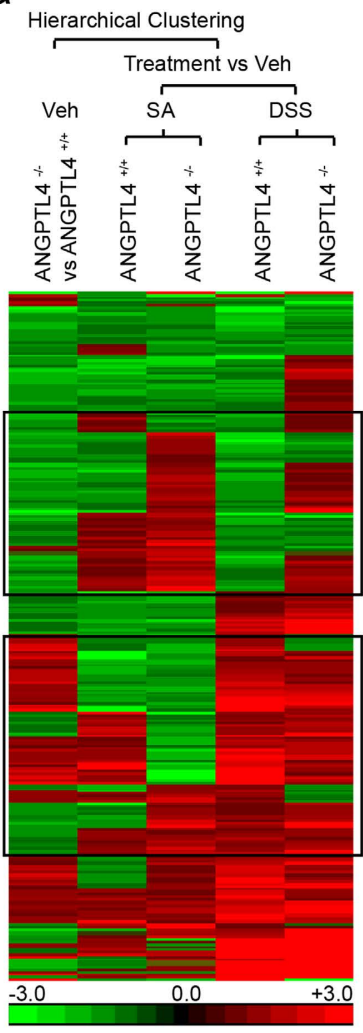

e

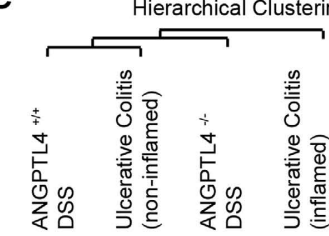

b

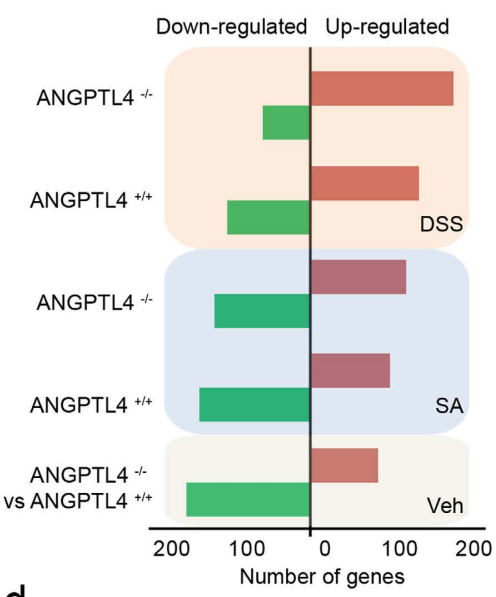

d

Gastrointestinal Diseases and Functions

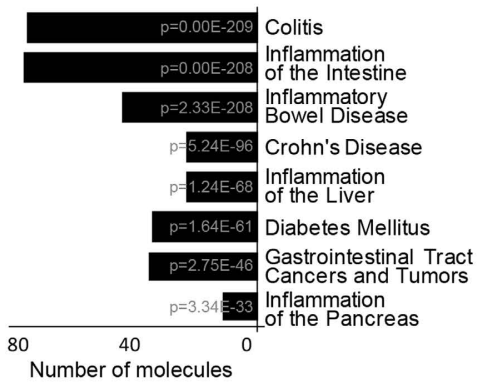

$f$

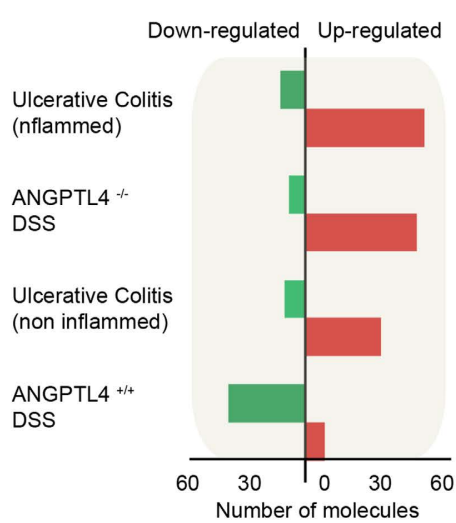

$\mathrm{h}$

Molecular and Cellular Functions

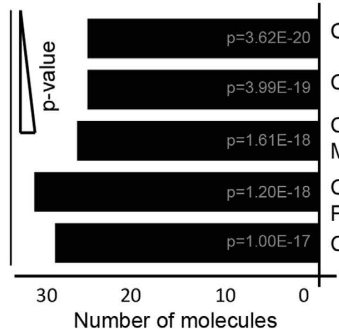

C

g Maintenance Proliferation

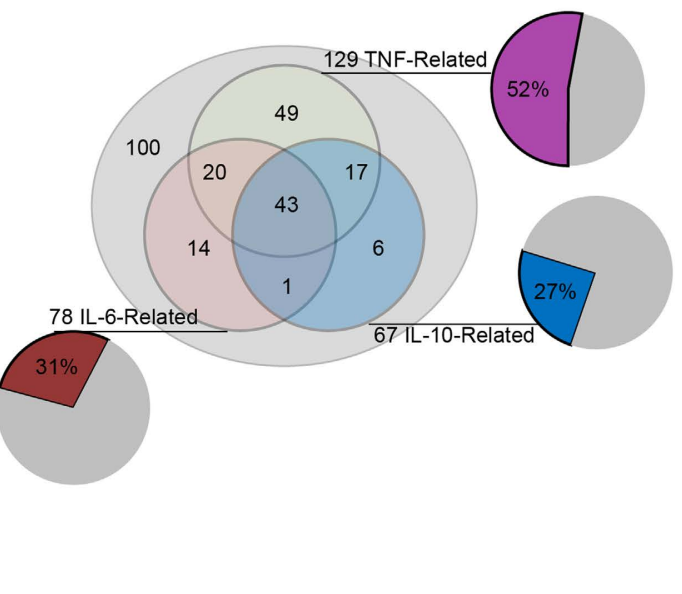

Immune Cell Trafficking and Functions
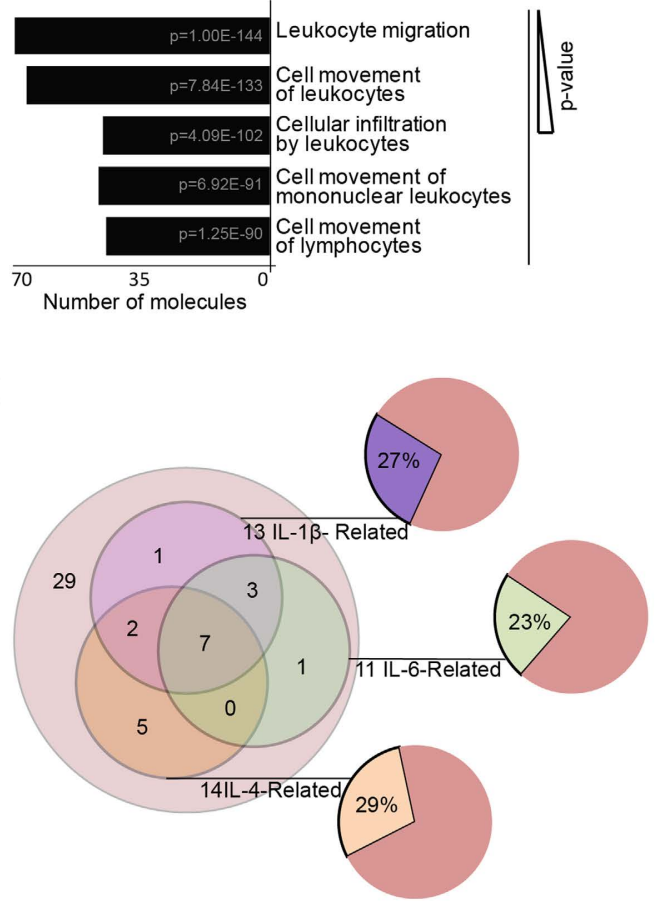

Biological Process and Functions

Cellular Movement Cellular Development Cellular Function and

Cellular Growth and Cell Death and Survival

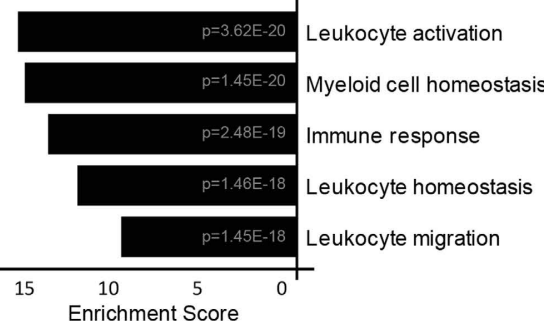

Figure 4. ANGPTL4 deficiency alters colonic inflammatory gene expression. (a,e) Using Partek Genomic Suite software, we generated a microarray heat map showing changes in gene expression among colon tissues from ANGPTL4 $4^{+/+}$and ANGPTL4 ${ }^{-1-}$ mice fed with Veh, DSS or SA (a) and among colons from ANGPTL4 ${ }^{+/+}$ and ANGPTL4 ${ }^{-1-}$ mice treated with DSS, inflamed and non-inflamed colon biopsies from ulcerative colitis patients (GSE9452) (e). Transcripts were estimated using a $\log _{2}$ transformation and subjected to unbiased ANOVA to detect differentially expressed genes between samples. Only genes with a fold change $<-1.2$ or $>1.2$ were of significance. The genes were then hierarchical clustered based on significance to generate heatmaps. For (a), among the 434 transcripts associated with "gastrointestinal diseases" identified with the IPA database, 
$63 \%$ were found to be commonly regulated across the three treatment groups. For (e), $32 \%$ of the transcripts associated with "gastrointestinal diseases" were found to be shared among the groups, with the gene profile from DSS-induced inflammation in ANGPTL4 ${ }^{-1-}$ mice most closely associated with the inflamed UC biopsies. (b,f) A larger number of up-regulated genes was correlated with an increasing severity of colonic inflammation in the microarray analysis. (c) Gene ontology analysis of gene clusters (black boxes in (a)) indicated that the major pathways included IL-6-, IL-10- and TNF-related pathways. (d) IPA analysis ranked colitis, inflammation of the intestine and inflammatory bowel disease as the most closely associated gastrointestinal diseases, with leukocyte migration and infiltration as the predominant functions. (g) Among the genes that were commonly regulated between human and mouse colon samples (black box), most genes encode for components of the IL$1 \beta$, IL-6 and IL-4 pathways. (h) The IPA database ranked cellular movement, development, function, growth and proliferation as the top molecular processes involved, along with immune processes such as leukocyte activation, myeloid cell homeostasis and immune responses being the most significant biological functions involved. The numbers in the bar graphs in $(\mathbf{d}, \mathbf{h})$ represent the p-values of the process in the GO analysis, with the smallest p-value being the most significant. An in-depth overview is described in Supplementary Fig. S4.

anti-inflammatory ( $\mathrm{HC}$ or $\mathrm{NaBu}$ ) stimuli. Notably, ANGPTL4 and TTP levels decreased when iCECs were treated with DSS, SA, IL-1 $\beta$ and TNF- $\alpha$ (Fig. 5b). Exposure to such pro-inflammatory stimuli increased the levels of CCL2, CCL11, CXC10 and IL-1 $\beta$ mRNA transcripts and decreased the levels of IL-10 and IL-17 mRNA transcripts (Supplementary Fig. 2d). Conversely, anti-inflammatory HC and NaBu increased both ANGPTL4 and TTP levels, prompting a decreased expression of CCL2, CCL11, CXCL10 and IL-1 $\beta$ concomitant with an increase in IL-10 and IL-17 (Fig. 5b and Supplementary Fig. 2d). Next, we examined the role of ANGPTL4 and TTP in mediating the abundance and stability of chemokines. iCECs were subjected to transient ANGPTL4 (iCE$\mathrm{C}_{\mathrm{ANGPTL} 4}$ ) or TTP knockdowns ( $\mathrm{iCEC}_{\mathrm{TTP}}$ ) and subsequently exposed to pro- or anti-inflammatory stimulation (Fig. 5c and Supplementary Fig. S2e-g, Tables S6 and 7). As a control, scrambled siRNA was used (iCEC Ctrl $_{1}$ ). Pro-inflammatory stimuli (DSS, SA, IL- $1 \beta$ and TNF- $\alpha$ ) increased CCL2, CCL11, CXCL10 and IL- $1 \beta$ expression but decreased IL-10 and IL-17 in iCEC $\mathrm{Ctrr}_{\text {. }}$. Conversely, $\mathrm{HC}$ and $\mathrm{NaBu}$ stimulation in $\mathrm{iCEC}_{\mathrm{Ctrl}}$ suppressed CCL2, CCL11, CXCL10 and IL-1 $\beta$ but heightened IL-10 and IL-17. The absence of ANGPTL4 (iCEC ANGPTL4 $_{4}$ )raised the basal levels of CCL2, CCL11, CXCL10, IL-1 $\beta$, IL-10 and IL-17 expression independent of the nature of stimulation. Interestingly, the CCL11, IL-10 and IL-17 levels surged independent of stimulation when TTP was ablated $\left(\mathrm{iCEC}_{\mathrm{TTP}}\right)$. Further investigation into chemokine stability demonstrated that the half-lives of CCL11, IL-10 and IL-17 mRNA transcripts, but not CCL2, IL-1 $\beta$ or CXCL10, were prolonged in iCEC $_{\text {ANGPTL4 }}$ and iCEC $_{\text {TTP }}$ (Fig. 5d). The decreased half-life of TTP in iCEC $_{\text {ANGPTL4 }}$, and not vice versa, also confirmed that ANGPTL4 positively regulates downstream TTP (Supplementary Fig. S2g). In an effort to understand immune cell infiltration, THP1-derived macrophages were co-cultured with iCECs $\left(\mathrm{iCEC}_{\mathrm{ANGPT}_{4}}, \mathrm{iCEC}_{\mathrm{TTP}}\right.$ and $\mathrm{iCEC}_{\mathrm{Ctrl}}$ ) in the presence of various treatments, and macrophage transwell migration was investigated. Consistent with the above results, we found that deficiency in ANGPTL4 and TTP was sufficient to increase chemotactic signals from epithelial cells and accelerate the migration of macrophages (Fig. 5e and Supplementary Fig. S2i). Taken together, our results demonstrate that ANGPTL4 is an important regulator that modulates the chemokine landscape and immune cell infiltration during inflammation. Our data also highlights the role of ANGPTL4 in attenuating inflammation simultaneously through TTP-dependent and independent pathways, although the mechanism for the latter remains to be elucidated.

ANGPTL4 up-regulates TTP via CREB and NF- $\kappa$ B. Full-length ANGPTL4 is proteolytically cleaved into N-terminal coiled-coil (nANGPTL4) and C-terminal fibrinogen-like (cANGPTL4) fragments ${ }^{31}$. To understand which fragment of ANGPTL4 is responsible for regulating TTP expression, we treated iCECs with recombinant (rh) cANGPTL4 and rh-nANGPTL4. This investigation showed that cANGPTL4 was potent in significantly up-regulating TTP expression (Supplementary Fig. 3a). Next, we sought to elucidate the mechanism whereby ANGPTL4 regulates TTP expression. We subjected rh-cANGPTL4-stimulated iCECs to a kinase inhibitor array screen to identify key signaling pathways involved in the cANGPTL4-mediated up-regulation of TTP (Supplementary Fig. 3b). TTP expression was increased when iCECs were treated with rh-cANGPTL4 (Supplementary Fig. 3b, left panel). Hence, we reasoned that kinase inhibitors that negated or inhibited the rh-cANGPTL4-mediated up-regulation of TTP mRNA transcripts indicated the involvement of those specific kinases in the selected signaling cascades (middle panel). Conversely, kinase inhibitors that did not attenuate the cANGPTL4-mediated up-regulation of TTP would indicate that those kinases were not involved in ANGPTL4-mediated signaling (right panel). Our results showed that inhibitors against CDK, JNK, MEK1/2, mTOR, PI3K 8 , TYK2, BTK, CK2, GSK3, PDK1 and Aurora A/B/C targeted key signaling regulators of cANGPTL4-mediated TTP transcription (Supplementary Fig. 3c). To avoid bias, all kinases that prevented the up-regulation of TTP expression under the stimulation of rh-cANGPTL4 were used to study how their availability impacted the overall level of inflammatory signaling. Using IPA, we further established that these signaling mediators resulted in the activation of downstream transcription factors, such as AP1 (cFos-cJun complex), CREB and NF- $\kappa B$ (Fig. 6a). Immunoblot analysis revealed that rh-cANGPTL4 increased the phosphorylation of CREB (pCREB) and NF- $\kappa B$ (pNF- $\kappa B$ ) but not phosphorylated cFos (pcFos) (Fig. 6b). In silico analysis identified putative CREB ( +455 to $+453 \mathrm{bp}), \mathrm{AP} 1(+388$ to $+395 \mathrm{bp})$ and NF- $\mathrm{KB}(+528$ to $+537 \mathrm{bp})$ transcription binding sites in the promoter of TTP (Fig. 6c).

To underscore the importance of CREB and NF- $\kappa B$ as active transcription factors mediating TTP expression upon cANGPTL4 stimulation, we inhibited the activity of CREB, NF- $\kappa B$ or both using siRNAs (iCEC RELA, $_{\text {, }}$ iCEC${ }_{\text {CREB }}$ and $\mathrm{iCEC}_{\text {RELA/CREB }}$ ) and specific kinase inhibitors (IKK-2, 666-15 and IKK-2/666-15), respectively. The highly 
a

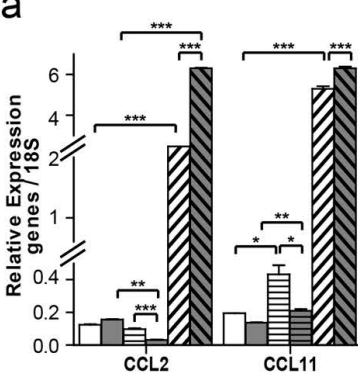

b

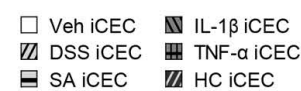

$\boxminus$ SA ICEC

III HC iCEC E NaBu iCEC

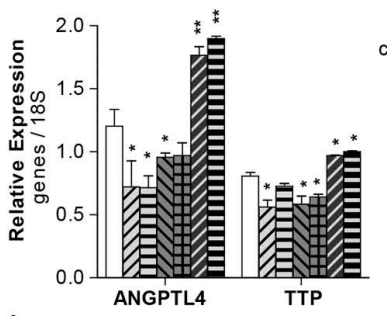

CANGPTL

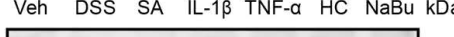

Veh DSS SA IL-1 $1 \beta$ TNF- $\alpha$ HC NaBu kD

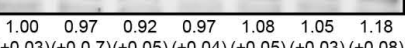

$( \pm 0.03)( \pm 0.0 .7)( \pm 0.05)( \pm 0.04)( \pm 0.05)( \pm 0.03)( \pm 0.08)$

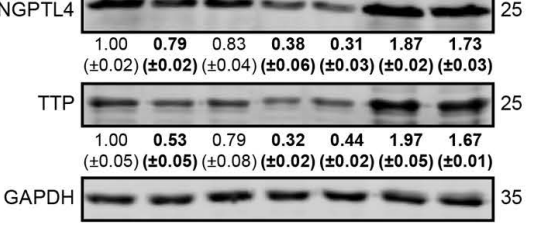

d
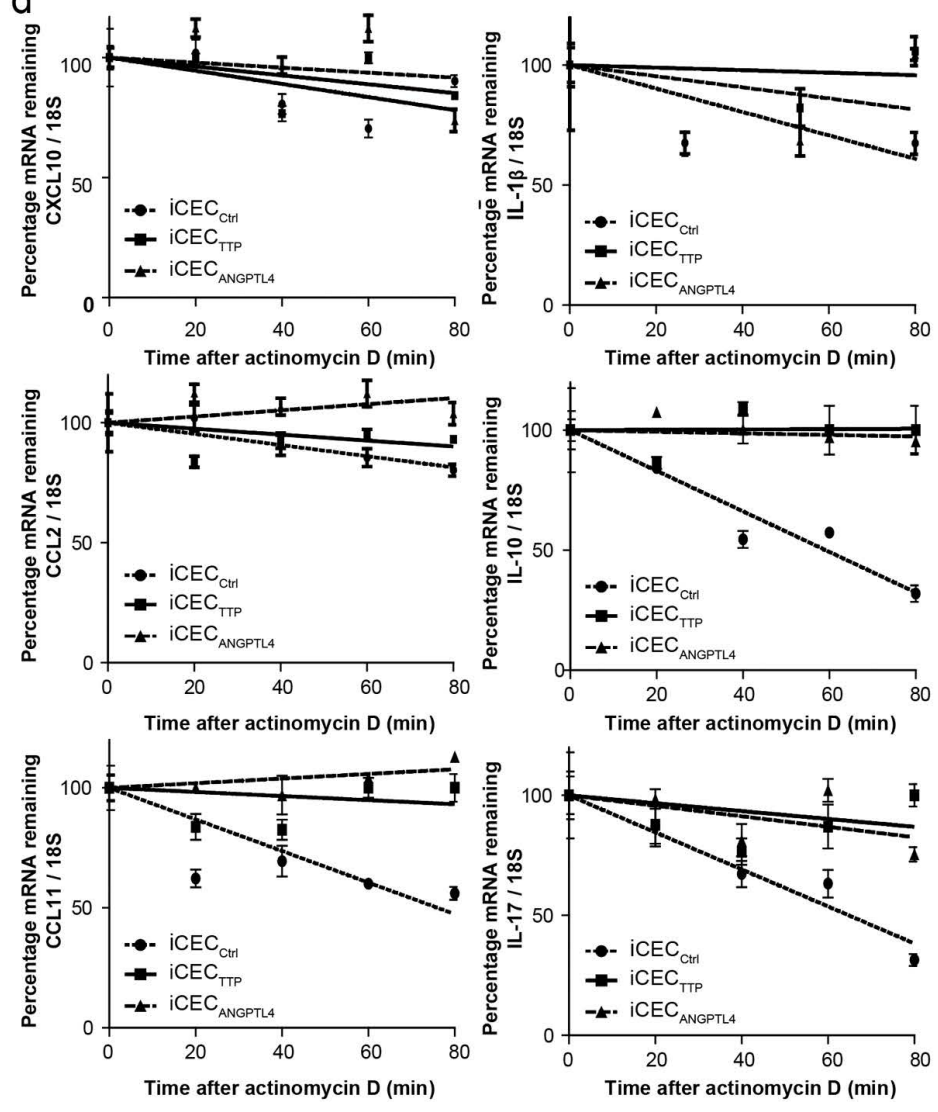

C
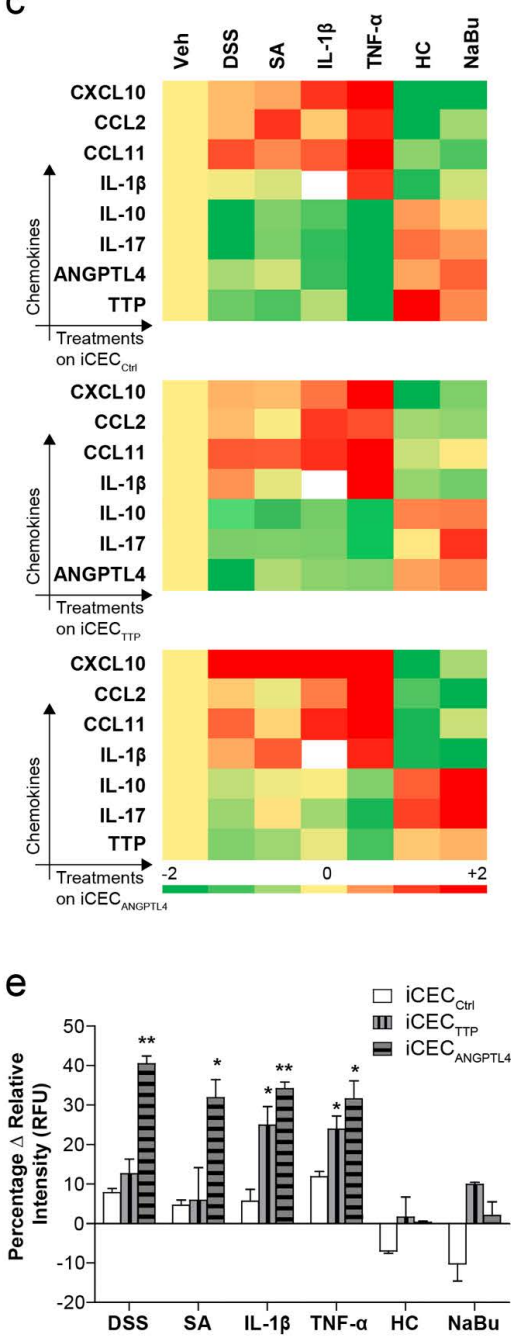

Figure 5. ANGPTL4 deficiency increases the infiltration of immune cells. (a) Relative mRNA levels of indicated chemokines in colon tissues from ANGPTL4 ${ }^{+/+}$and ANGPTL4 ${ }^{-1-}$ mice treated with Veh, DSS or SA. (b) Relative expression (left panel) and immunoblot analysis (right panel) of ANGPTL4 and TTP levels in iCECs when stimulated with DSS, SA, IL-1 $\beta$, TNF- $\alpha, \mathrm{HC}$ or NaBu. (c) Heatmap displaying log-transformed relative expression levels of indicated chemokine mRNA transcripts in iCEC $_{\text {Ctrl }}$, iCEC $_{\text {TTP }}$ and iCEC $_{\text {ANGPTL4 }}$ when stimulated with DSS, SA, IL-1 $\beta$, TNF- $\alpha$, HC or NaBu. (d) Decay curves of IL-1 $\beta$, IL-10, IL-17, CCL2, CCL11 and CXCL10 in $\mathrm{iCEC}_{\mathrm{ANGPTL}}, \mathrm{iCEC}_{\mathrm{TTP}}$ or $\mathrm{iCEC}_{\mathrm{Ctr}}$, following actinomycin $\mathrm{D}$ treatment after $\mathrm{NaBu}$ stimulation. (e) Transwell migration assay measuring the percentage change in relative fluorescence intensity of THP1 migration in response to chemokines secreted by $\mathrm{iCEC}_{\mathrm{Ctr}}, \mathrm{iCEC}_{\mathrm{TTP}}$ and $\mathrm{iCEC}_{\mathrm{ANGPTL} 4}$ (bottom panel). Five independent experiments $(n=5)$ were performed in triplicate. The Mann-Whitney $U$ test was used. ${ }^{*} \mathrm{p}<0.05$, ${ }^{* *} \mathrm{p}<0.01,{ }^{* *} \mathrm{p}<0.001$. 
a

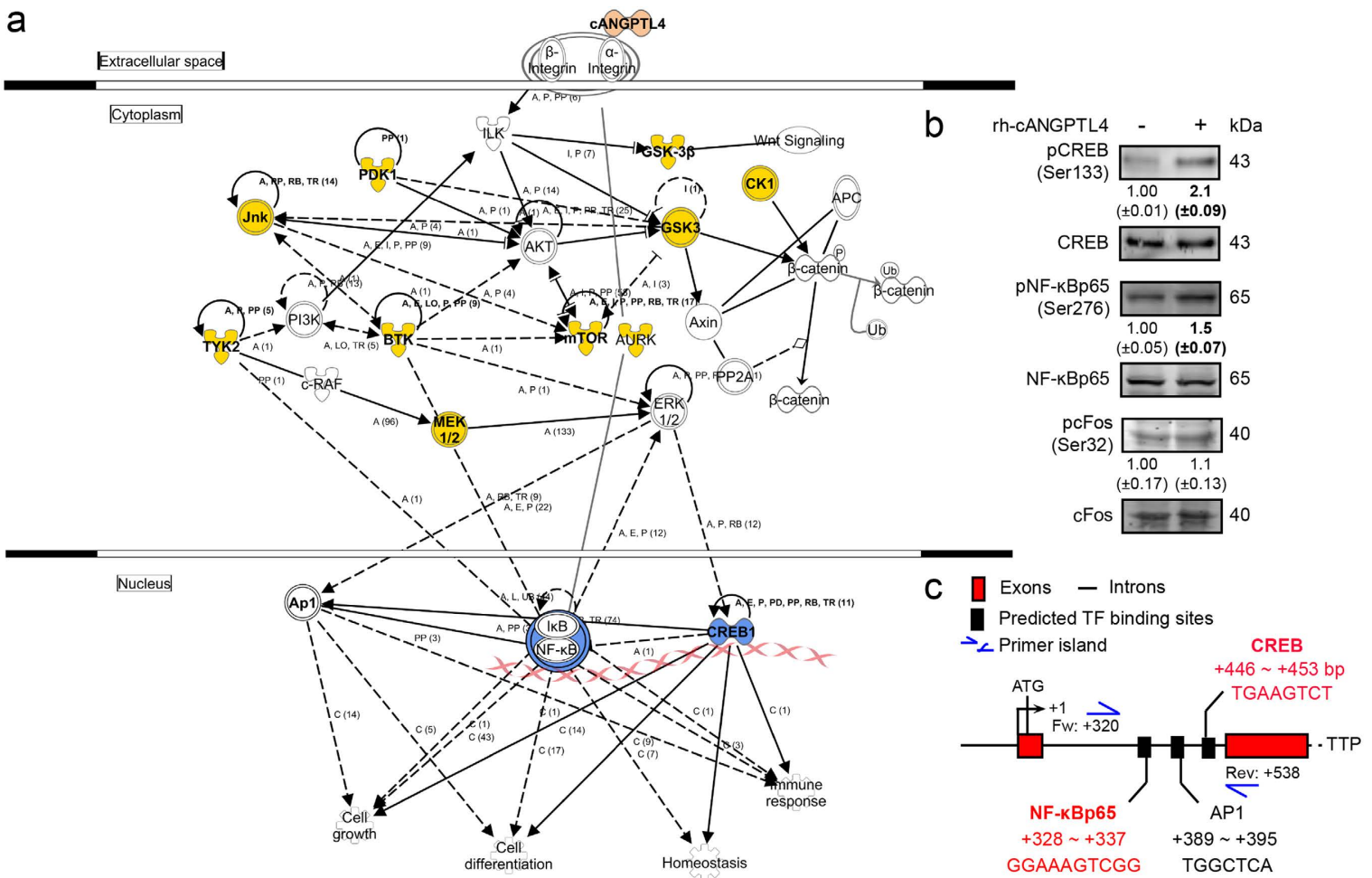

d
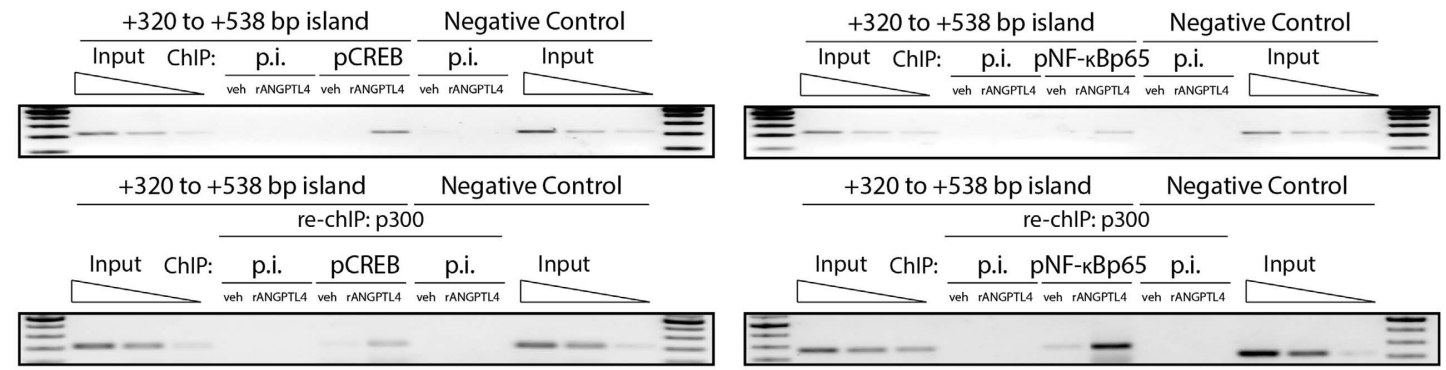

e

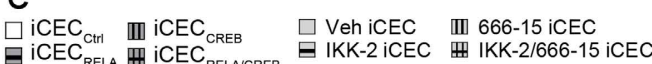

日 ICE $C_{\text {RELA }}$ 田 ICEC

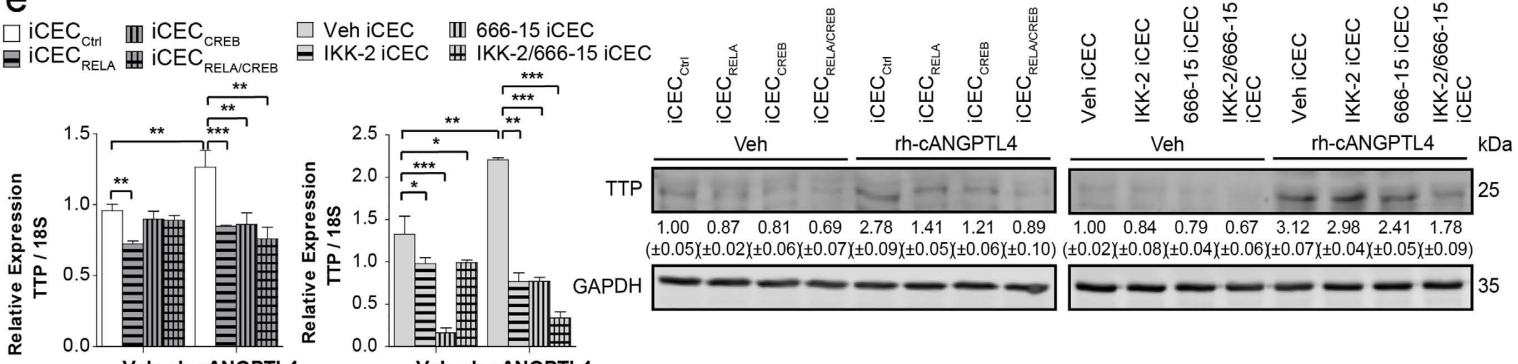

f
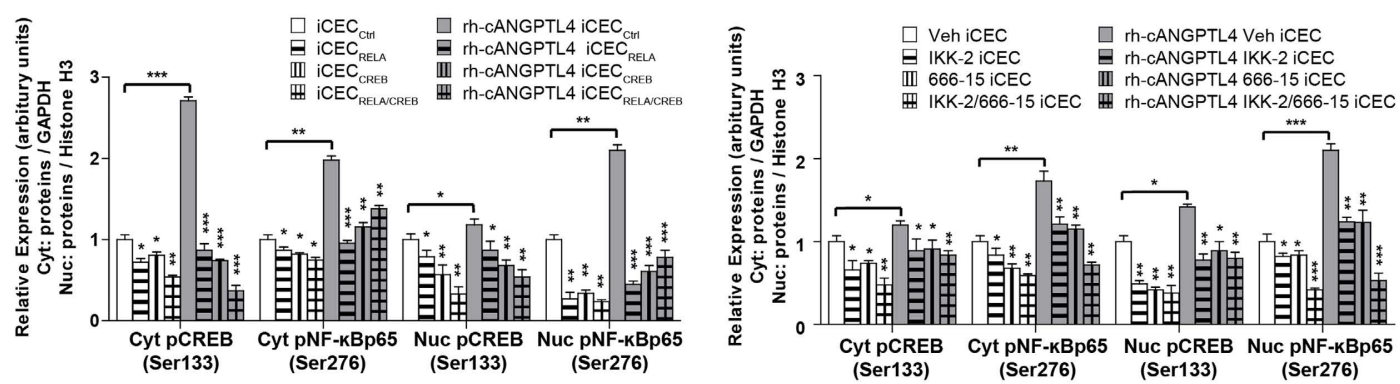

Figure 6. ANGPTL4 up-regulates TTP expression via CREB and NF- $\kappa B$. (a) IPA identified CREB, NF- $\kappa B$ and AP-1 as potential transcription factors that regulate the expression of TTP when stimulated with rhcANGPTL4. IPA-assisted pathways were mapped following experimental screens using a kinase inhibitor array. (b) Immunoblot analysis revealed an increase in the phosphorylation of CREB (pCREB) and NF- $\kappa B$ p65 (pNF- $\kappa \mathrm{B}$ ) but not cFos (pcFos) after rh-cANGPTL4 stimulation. (c) A schematic illustration of the promoter of the human TTP gene. The putative transcription factors binding sites were determined in silico online with the 
Jaspar database. The relative location of the ChIP primers was indicated. (d) Chromatin Immunoprecipitation (ChIP) and re-ChIP using pCREB or pNF- $\kappa \mathrm{B} / \mathrm{p} 65$ and coactivator $\mathrm{p} 300$ antibodies in iCECs that were stimulated with rh-ANGPTL4. (e) Relative expression and immunoblot analysis of TTP for iCECs repressed of CREB and/or NF- $\kappa$ B activity using siRNA (iCEC Ctrl $_{1}, \mathrm{iCEC}_{\mathrm{RELA}}, \mathrm{iCEC}_{\mathrm{CREB}}$ and $\mathrm{iCEC}_{\mathrm{RELA} / \mathrm{CREB}}$ ) and specific kinase inhibitors (Veh, IKK-2, 666-15 and IKK-2/666-15) in the absence and presence of rh-cANGPTL4. (f) Densitometry analysis showing the relative expression of pCREB and pNF- $\kappa$ Bp 65 in nuclear and cytoplasmic fractions of iCECs as treated in (e), normalized against Histone $\mathrm{H} 3$ and GAPDH for nuclear and cytoplasmic fractions, respectively, for Supplementary Fig. 3h. The Mann-Whitney U test was used. Three independent experiments $(\mathrm{n}=3)$ were performed.

potent CREB inhibitor (666-15) hinders the interaction of KID-KIX, which is essential for CREB-dependent gene transcription $^{32}$, while the IKK-2 inhibitor IV (IKK-2) selectively targets IKK-2 and retards downstream p65NF-kB signaling. In both instances, the lack of either CREB or NF- $\kappa B$ was sufficient to reduce TTP expression in iCECs even in the presence of rh-cANGPTL4 (Fig. 6e and Supplementary Fig. S3g). We also observed reduced pCREB and $\mathrm{pNF}-\kappa \mathrm{B}$ levels in nuclear and cytoplasmic fractions of iCECs during cANGPTL4-stimulation, suggesting that the nuclear translocation of pCREB and pNF- $\kappa B$ was severely compromised (Fig. $6 \mathrm{f}$ and Supplementary Fig. 3h). In conclusion, our data suggests that the CREB and NF- $\kappa B$ transcription factors play irreplaceable roles in the ANGPTL4-mediated, TTP-dependent signaling axis.

\section{Discussion}

The epithelial cells that line the gastrointestinal tract serve as a physiological barrier that prevents the invasion of pathogens and a selective conduit of luminal signals to the host. When harmful immunogens and pathogens are detected, epithelial cells produce chemoattractants to initiate the infiltration of immune cells into the mucosa. Our findings highlight the importance of colonic epithelial cell-secreted ANGPTL4 as a prospective regulator that alters the chemokine landscape in the colon to influence downstream inflammation. We showed that ANGPTL4-deficient mice were more susceptible to acute DSS and SA exposure. 16S metagenomic sequencing indicated little difference in microbe composition between ANGPTL4 ${ }^{+/+}$and ANGPTL4 ${ }^{-1-}$ mice that could account for the exacerbated inflammatory response in ANGPTL4 ${ }^{-1-}$ mice. Bone marrow transplant study emphasized the intrinsic role of epithelial-derived ANGPTL4 in regulating the inflammatory landscape in the colon. Using human colonic epithelial cells, we further showed that ANGPTL4 regulated the expression of TTP, an mRNA destabilizing agent, via the activation of CREB and NF- $\kappa$ B. The absence of ANGPTL4 or TTP prolonged the mRNA half-life of a specific subset of chemokines.

An exuberant and protracted inflammatory response contributes to the development of many gastrointestinal disorders. Infiltrating macrophages are the major cellular components of this inflammatory response. As the initial cellular barrier, the colonic epithelium plays an important role in the recruitment of inflammatory cells to the mucosa. We showed that ANGPTL4-knockout mice fed DSS or SA exhibited a greater colonic inflammatory response, which was associated with greater infiltration of immune cells when compared with wild-type mice fed similar treatments. Similarly, pro-inflammatory stimuli such as DSS, SA, IL- $1 \beta$ and TNF- $\alpha$ suppressed the expression of ANGPTL4 and TTP in iCECs, whereas anti-inflammatory stimuli such as $\mathrm{HC}$ and $\mathrm{NaBu}$ increased its expression. In support of these findings, previous studies showed that anti-inflammatory glucocorticoid treatment boosted TTP expression in lymphocytes ${ }^{33}$, while the synthetic glucocorticoid dexamethasone increased TTP levels in A549 cells ${ }^{34}$ as well as ANGPTL4 levels in hepatocytes ${ }^{35}$ and adipocytes ${ }^{36}$. Although the exact pathways remain to be elucidated, dietary $\mathrm{NaBu}$ was also found to stimulate ANGPTL4 expression via a PPAR-independent pathway ${ }^{37}$. HC has also been demonstrated to up-regulate IL-10 and IL-17 levels in natural killer cells to improve pneumonia survival rates ${ }^{38}$, although no detailed mechanism was reported. Interestingly, our findings cement the importance of ANGPTL4 in regulating the general inflammatory landscape in the gastrointestinal tract. Although other ANGPTL4-dependent but TTP-independent signaling mechanisms remain to be identified, we demonstrated that ANGPTL4 modifies the availability of a subset of chemokine signals that alter the ease of immune cell infiltration through a TTP-dependent signaling circuit.

Confluent iCECs mimic an intact gastrointestinal mucosal layer and express low basal levels of ANGPTL4 and TTP. Many previous studies have utilized cancerous lines, such as HT-29, Caco-2 and HCT-116 cells, as surrogates for an in vitro gastrointestinal model ${ }^{39,40}$. Potentially, this ANGPTL4-dependent mechanism may be impaired or altered in cancerous lines, as the expression of ANGPTL4 is known to be elevated in many types of cancer ${ }^{7}$. We further established that iCECs secreted ANGPTL4, which acted in an autocrine manner to induce the expression of TTP. We previously showed that secreted ANGPTL4 bound and activated integrin-mediated pathways ${ }^{41}$. Indeed, TTP is an important factor that contributes to mediating, modulating and attenuating inflammatory responses. Using the combination of an unbiased kinase inhibitor screen assay, along with ChIP and re-ChIP experiments, our current work unveiled multiple signaling conduits whereby ANGPTL4 stimulates TTP expression, resulting in CREB and NF- $\kappa B$ activation. Interestingly, BTK and Aurora inhibitors were identified to significantly inhibit the ANGPTL4-dependent up-regulation of TTP, suggesting that they exert dominant roles in this signaling cascade. Lending support for a role for Aurora in NF-kB activation, Katsha A et al. also reported that Aurora kinase A promoted and sustained inflammation through NF- $\kappa B$, leading to tumor formation ${ }^{42}$. Again, the importance of Aurora in the ANGPTL4-mediated up-regulation of TTP may be compromised when studied in cancer cell lines. Our study also emphasizes a potential role for Aurora kinases in regulating inflammation. Similarly, other studies have shown that cancer cells that express low levels of TTP are correlated with a genetic signature of low expression of CREB-related target genes ${ }^{43}$. Numerous studies have also shown that TTP binds to AU-rich sequences at the $3^{\prime}$-UTRs of specific mRNA transcripts such as TNF- $\alpha^{44}$, IFN- $\gamma^{45}$, IL-10 and IL- $17^{46}$. In 
agreement with these previous reports, we showed that the mRNA half-lives of specific inflammatory chemokines were prolonged in the absence of ANGPTL4 and TTP.

The pathogenesis of inflammatory bowel disease in humans is complex and has a multifactorial and diverse aetiology ${ }^{47}$. Dysbiosis has been associated with inflammation and impaired mucosal immune function in intestine of humans ${ }^{48-50}$. Our $16 \mathrm{~S}$ sequencing revealed little difference in microbe composition between two genotypes that can account for the exacerbated colonic inflammatory response in ANGPTL4 ${ }^{-1-}$ mice. In this respect, the extent to which ANGPTL4 expression defines the colonic microbiota profiles remains to be delineated. It is also conceivable that mechanisms other than ANGPTL4-TTP-mediated chemokine mRNA stability might be involved. Our microarray analyses showed that $\operatorname{Lrg} 1$ and Gpx2 expression was up-regulated by 6- and 4-fold, respectively, in the colons of ANGPTL4 ${ }^{-1-}$ mice compared with wild-type counterparts. $\operatorname{Lrg} 1$ is proposed to be a possible biomarker for the diagnosis of ulcerative colitis ${ }^{51}$. Both Lrg1 and Gpx2 proteins are up-regulated during the acute stages of pediatric appendicitis ${ }^{52}$ and colitis ${ }^{53}$, respectively. These data suggest that additional ANGPTL4-dependent mechanisms may contribute to the severity of colitis in ANGPTL4 ${ }^{-1-}$ mice. Furthermore, ANGPTL4 has been implicated in many inflammation-associated pathologies. Although not explored in the present study, the mechanism whereby ANGPTL4 may be targeted to influence cell infiltration via TTP-mediated chemokine mRNA stability could be applicable to these pathologies.

\section{Materials and Methods}

Antibodies and Reagents. Antibodies against c-Fos (\#2250), phospho-c-Fos (Ser32; \#5348), Elk-1 (\#9182), phospho-Elk-1 (Ser383; \#9186), CREB (\#48H2) and phospho-CREB (Ser133; \#9198) were from Cell Signaling

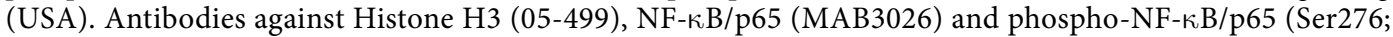
AB3375) were from Merck Millipore, USA. Antibodies against Ly-6G (\#127602 and \#127614), CD68 (\#137002) were from Biolegend, USA. FACS antibodies (APC-/FITC-conjugated) against CD11b, CD45, F4/80 were from Miltenyi Biotec, USA. Anti-CD4- and anti-CD8a- conjugated microbeads (\#130-049-201, \#130-049-401) was purchased from Miltenyi Biotec, USA. Anti-TTP (sc-14030, Santa Cruz, USA), active/cleaved Caspase 3 (NB10056113, Novus Biological, USA),

ChIP-grade antibodies against NF- $\kappa \mathrm{B} / \mathrm{p} 65$ (ab7970) and CREB (ab31387) were from Abcam, UK and p300 (RW128) from Upstate Biotechnology, USA. IRDye ${ }^{\circledR} 680$ LT goat anti-rabbit IgG, goat anti-mouse IgG and donkey anti-goat IgG were purchased from Li-Cor Biosciences, USA. Dextran sulfate sodium salt (DSS; 36000 to 50 000M.Wt) was from Affymetrix, USA (\#14489). Recombinant human cANGPTL4 (rh-cANGPTL4) and anti-ANGPTL4 antibodies were produced in-house as previously described ${ }^{24}$. Recombinant human nANGPTL4 (rh-nANGPTL4; 8249-AN, RnD Systems), IL-1 $\beta$ (ab9617, Abcam), TNF- $\alpha$ (\#717904, BioLegend) and hydrocortisone (CAS 50-23-7, Calbiochem). All other chemicals were purchased from Sigma-Aldrich.

Animals. ANGPTL4 ${ }^{+/-}$C57BL/6J mice were acquired from the Mutant Mouse Regional Resource Center (MMRRC) and were generated by Genentech. The mice were crossed to produce ANGPTL4 ${ }^{+/+}$and ANGPTL4 ${ }^{-1-}$ offspring. DNA from mouse ear clippings was isolated using the KAPA Express Extract reactions and genotyped using Mouse Genotyping HotStart in accordance with the manufacturer's recommendations (KAPA Biosystems, USA).

The PCR products were visualized on $2 \%$ agarose gels stained with SYBR Safe (ThermoFisher Scientific, USA). In the DSS treatment study, age-matched ANGPTL4 ${ }^{+/+}$and ANGPTL4 ${ }^{-1-}$ males $(\mathrm{n}=20)$ were treated with $5 \%$ DSS in the drinking water for 8 days; the DSS was changed every 2 days. In the SA study, mice $(n=20)$ were fed with a $15 \%$ SA: $85 \%$ ground chow $(w / w)$ diet for 8 days. The disease activity index (DAI) was assessed every alternate day, while macroscopic and histological scorings were performed at the experimental endpoint. The scoring criteria are available in Supplementary Tables S1-3. Protein and RNA samples were collected by scraping colonic epithelial cells. Animal experiments were approved by and carried out in accordance with the guidelines of Nanyang Technological University's Institutional Animal Care and Use Committee (NTU, IACUC, ARF SBS/ NIE-A0321 and ARF SBS/NIE-A0324), Singapore.

Cell Culture. SV40-immortalized human colon epithelial cells (iCECs; T0570; ABM Canada) were cultured in Prigrow III Medium (ABM Canada) in collagen-coated flasks and maintained at $5 \% \mathrm{CO}_{2}$ at $37^{\circ} \mathrm{C}$. Confluent iCECs were stimulated with either $1 \mu \mathrm{g} / \mathrm{mL}$ DSS, $500 \mu \mathrm{M} \mathrm{SA}, 10 \mathrm{ng} / \mathrm{mL} \mathrm{IL}-1 \beta, 10 \mathrm{ng} / \mathrm{mL}$ TNF- $\alpha, 0.4 \mathrm{ug} / \mathrm{mL} \mathrm{HC}$ or $2 \mathrm{mM} \mathrm{NaBu}$ for $6 \mathrm{~h}$ prior to harvesting. The siRNA knockdown against ANGPTL4, CREB, RELA or TTP was accomplished using Dharmacon ON-TARGETplus siRNA (Supplementary Table S8) according to the manufacturer's protocol (Thermo Scientific, USA). Inhibition of the transcription factors CREB and IKK were carried out using $0.1 \mu \mathrm{M}$ CREB inhibitor (666-15; \#5661; Tocris Bioscience, UK) and/or $1 \mu \mathrm{M}$ IKK-2 inhibitor IV (IKK-2;

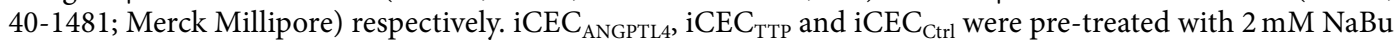
for $2 \mathrm{~h}$, followed by $10 \mu \mathrm{g} / \mathrm{mL}$ actinomycin $\mathrm{D}$ for half-life measurements. In vitro experiments were carried out in triplicate.

RNA Extraction and Real-time Quantitative PCR. RNA was extracted using TRIzol (Invitrogen, USA) and was reverse transcribed using the iScript ${ }^{\mathrm{TM}}$ Reverse Transcription Supermix according to the manufacturer's recommendations (Bio-Rad, USA). Quantitative PCR (qPCR) was conducted as previously described ${ }^{54}$. Primer sequences are presented in Supplementary Table S9. The relative expression levels of the respective mRNAs described in this manuscript were normalized against those of the housekeeping ribosomal 18S RNA.

Microarray. RNA was transcribed into cDNA using an Applause WT-Amp ST System in accordance with the manufacturer's recommendations (NuGEN Technologies, USA). cDNA was purified using a MinElute Reaction Cleanup Kit (Qiagen, USA), fragmented and labeled using an Encore Biotin Module kit (NuGEN Technologies, 
USA). Labeled cDNA molecules were hybridized using the Affymetrix hybridization master mix and injected onto GeneChip Mouse Gene 1.0 ST Array gene chips (Affymetrix, USA) for scanning. Partek Genomic Suite (Partek Inc., USA) and Ingenuity Pathway Analysis (IPA) software were used to perform analyses, an overview of which is available in Supplementary Fig. S4. The human colonic mucosal samples used for the comparative analyses in Fig. 3e-h were published by Olsen et al. under the GEO accession number GSE9452 ${ }^{55}$. The microarray data (accession number GSE78500) have been deposited in the GEO database.

Protein Extraction and Western Blot Analysis. Proteins were extracted in ice-cold lysis buffer $(20 \mathrm{mM}$ $\mathrm{Na}_{2} \mathrm{H}_{2} \mathrm{PO}_{4}, 250 \mathrm{mM} \mathrm{NaCl}, 1 \%$ Triton-X, $0.1 \%$ SDS, $1 \mathrm{mM}$ PMSF, and $200 \mu \mathrm{M}$ sodium orthovanadate) as previously described ${ }^{44}$. Equal amounts of proteins were resolved using SDS-PAGE gels and electro-transferred to low-fluorescence PVDF membranes (IPFL00010; Merck Millipore, USA). The cytoplasmic and nuclear fractions were isolated using the NE-PER Nuclear and Cytoplasmic Extraction Reagents (\#78833; Thermo Scientific). Briefly, all protein samples for western blot analysis performed in this manuscript were first normalized using the NanoDrop 3300 Spectrophotometer (Thermo Scientific) prior to the ensuing downstream analyses. All loading controls for immunoblot analyses were obtained from the same sample. Membranes were blocked with $0.5 \times$ Odyssey Blocking Buffer (LI-COR Biotechnology, USA) and probed with the respective primary antibodies overnight at $4{ }^{\circ} \mathrm{C}$ in $1 \times$ Blocking Buffer with $0.1 \%$ Tween-20. Membranes were washed thrice in TBST $(50 \mathrm{mM}$ Tris $\mathrm{HCl}, \mathrm{pH} 7.6,150 \mathrm{mM} \mathrm{NaCl}, 0.1 \%$ Tween-20) for $5 \mathrm{~min}$ each and incubated for $1 \mathrm{~h}$ with the respective secondary antibodies in $1 \times$ Blocking Buffer at room temperature. Each membrane was washed thrice in TBST and then air-dried. Images were analyzed using a CLx scanner and Image Studio V2.1 (LI-COR Biosciences, USA). All membranes were first probed for target proteins before being re-probed for GAPDH housekeeping protein. Densitometry analyses were processed using Image (NIH, USA). Densitometry values are presented under each blot, with values in bold representing significance $(\mathrm{p}<0.05)$, or in graphs (for Fig. $4 \mathrm{e}$ and Supplementary Fig. 3h). The LEGENDplex Multi-Analyte Flow Assay Kit was used to measure the protein concentrations of targets (CCL2, CCL11, CXCL10, IL-1ß, IL-10 and IL-17) for both mouse and humans and was customized through Biolegend, USA and performed in accordance with the manufacturer's recommendations.

Tissue Processing, H\&E, Immunofluorescence and TUNEL Staining. Tissues were harvested and fixed in $4 \%$ paraformaldehyde overnight at $4{ }^{\circ} \mathrm{C}$ and subsequently were dehydrated and embedded in paraffin as previously described ${ }^{56}$. Tissues were rehydrated and stained with hematoxylin and eosin (H\&E) according to the manufacturer's protocol (Sigma-Aldrich, USA). Slides were mounted with Fluka Eukitt quick-hardening mounting medium (Sigma-Aldrich, USA). Images were obtained using a Zeiss Axiovert 200M microscope with a Zeiss FLUAR 10x/0.5 NA objective and PALMRobo V4.3 software (Carl Zeiss, Germany).

The TUNEL assay was performed in accordance with the manufacturer's protocol (Roche). For immunofluorescence analyses, tissue sections were rehydrated and incubated in 5\% normal goat serum (NGS) for $1 \mathrm{~h}$ and then incubated with the primary antibodies in $3 \%$ NGS overnight at $4{ }^{\circ} \mathrm{C}$. Subsequently, sections were washed and then incubated with respective secondary Alexa Fluor 594-conjugated antibodies (Invitrogen) for 1 hour at room temperature. Slides were then mounted with Hoechst 33342 dye (Life Technologies, USA). Images were obtained using a LSM 710 confocal microscope with a Zeiss EC Plan-NEOFLUAR 20x/0.5 NA objective and were analyzed using ZEN 2012 Blue Edition software (Carl Zeiss, Germany).

Tissue Dissociation and Flow Cytometry. Mouse colon tissues from various treatment groups were first homogenized into single-cell suspensions using the gentleMACS Dissociator (Miltenyi Biotec, USA) in DMEM containing $1 \mathrm{mg} / \mathrm{mL}$ collagenase- 3 and 40 units $/ \mathrm{mL}$ DNase 1 . Homogenates were filtered through a $70 \mu \mathrm{m}$ nylon cell strainer, washed and finally resuspended in PBS blocking buffer containing 3\% FBS. The resultant suspension was then incubated with the respective FITC-/APC- conjugated antibodies on ice. Samples were washed twice and resuspended in PBS, and were then subjected to FACS analysis on the Accuri C6 Flow Cytometer (BD Biosciences, USA). All FACS analyses were performed for 5000 events.

Transwell Migration Assay. THP1 monocytes were differentiated in $100 \mathrm{ng} / \mathrm{mL}$ TPA complete medium for $48 \mathrm{~h}$ and allowed to recover for $24 \mathrm{~h}$ on transwell inserts. Respective siRNA knockdowns were performed on iCECS ( $\mathrm{iCEC}_{\mathrm{ANGPTL}}, \mathrm{iCEC}_{\mathrm{TTP}}$ and $\mathrm{iCEC}_{\mathrm{Ctrl}}$ ). Inserts containing differentiated THP1 cells were then introduced to the transfected iCECs and exposed to various pro- and anti- inflammatory stimuli for $10 \mathrm{~h} \mathrm{(Fig.} \mathrm{3e).} \mathrm{Inserts}$ were then washed with PBS twice and fixed in $1 \%$ glutaraldehyde for $10 \mathrm{~min}$, rinsed with PBS and stained with SYTO 60 (Thermo Fisher, USA) for $30 \mathrm{~min}$. Cotton buds were used to remove all unmigrated cells trapped in the upper chamber of the inserts. Inserts were rinsed again in PBS before the quantification of fluorescence using the CLx scanner and Image Studio V2.1 (LI-COR Biosciences, USA). Relative fluorescence as a readout for cellular migration was calculated by normalizing the intensities between test wells (THP1 and iCECs) and control wells (THP1 without iCECs).

Chromatin Immunoprecipitation (ChIP) and Re-ChIP. ChIP and re-ChIP experiments were performed as described previously ${ }^{57}$. In addition, samples were subjected to washes with increased stringencies: twice each with the low (1\% Triton X-100, $0.1 \%$ SDS, $2 \mathrm{nM}$ EDTA, $20 \mathrm{mM}$ Tris- $\mathrm{HCl}, 150 \mathrm{mM} \mathrm{NaCl}$ at $\mathrm{pH} 8.1)$ and high salt ( $1 \%$ Triton X-100, $0.1 \%$ SDS, $2 \mathrm{nM}$ EDTA, $20 \mathrm{mM}$ Tris- $\mathrm{HCl}, 500 \mathrm{mM} \mathrm{NaCl}$ at $\mathrm{pH}$ 8.1) wash buffers. Primer sequences are presented in Supplementary Table S9.

Kinase Inhibitor Array. Immortalized human colon cells were subjected to treatment with 95 kinase inhibitors (SYN-2103; SYNkinase, Australia) in the presence or absence of rh-c-ANGPTL4 $(8 \mu \mathrm{g} / \mathrm{mL})$ for $6 \mathrm{~h}$ (Supplementary Table S10). RNA was then isolated and reverse transcribed as described above. 
16S Metagenomics Sequencing. Post-weaned, age- and gender-matched ANGPTL4 ${ }^{-1-}$ or ANGPTL4 ${ }^{+/+}$ mice were used. Fresh fecal pellets were collected before and after DSS treatment. Bacterial genomic DNA was isolated from feces using the FASTDNA spin kit (\#116570200, MP Biomedicals, USA). 16S sequencing of the 16S V4 variable region was performed by SeqMatic LLC, USA using the Illumina MiSeq sequencing platform.

Bone Marrow Transplant Model. BMT was performed using bone marrow cells isolated from donor mice (ANGPTL4 ${ }^{-/-}$or ANGPTL4 ${ }^{+/+}$) and transplanted into recipient $\gamma$-irradiated ANGPTL4 ${ }^{+/+}$wild-type mice (WT IR; $\mathrm{n}=6$ per group). Recipient WT mice were kept on acidified water ( $\mathrm{pH} 3.0$ ) over the course of the BMT. WT mice were medicated with sulfamethoxazole $(40 \mathrm{mg} / \mathrm{kg})$ and trimethoprim $(8 \mathrm{mg} / \mathrm{kg})$ in oral suspension for one week prior to and after $\gamma$-irradiation $(9.5 \mathrm{~Gy}$ ) using the Biobeam 8000 (Gamma-Service Medical $\mathrm{GmbH}$, Germany). Red blood cells from donor bone marrow cells were lyzed $\left(0.89 \% \mathrm{NH}_{4} \mathrm{Cl}, 0.1 \mathrm{mM}\right.$ EDTA, $\mathrm{pH}$ 7.2). Residual bone marrow cells were washed with PBS and filtered through $30 \mu \mathrm{M}$ nylon cell strainer before depleting $\mathrm{CD}^{+}$and $\mathrm{CD}^{+}$cell populations using the QuadroMACS kit (\#130-091-051, Miltenyi Biotec, USA). Approximately $10^{7} \mathrm{CD}^{+}$and $\mathrm{CD} 8^{+}$depleted bone marrow cells were introduced to WT IR mice via retro-orbital injection. Chimera mice [BMT $\left(\right.$ ANGPTL4 ${ }^{+/+}$) and BMT $\left(\mathrm{ANGPTL}^{-/-}\right)$] were allowed to recover for 4 -weeks before $2 \%$ DSS treatment for 8 days. Bone marrow reconstitution was determined using genotype PCR (Supplementary Table S11). PCR products were visualized on 2\% agarose gels tinted with SYBR Safe (ThermoFisher Scientific, USA). Cell count was numerated using the automated ADAM-MC cell counter (NanoEntek, USA). Cells from the spleen and colon were analyzed for immune cell infiltration using FACS (BD LSRFortessa X-20).

Statistical Analysis. Statistical analyses were performed using two-tailed Mann-Whitney U tests. P-values $<0.05$ denote statistically significant differences between means; ${ }^{*} \mathrm{p}<0.05 ;{ }^{* *} \mathrm{p}<0.01$ and ${ }^{* * *} \mathrm{p}<0.001$. Values are expressed as the means \pm standard error.

\section{References}

1. Liddiard, K., Rosas, M., Davies, L. C., Jones, S. A. \& Taylor, P. R. Macrophage heterogeneity and acute inflammation. Eur J Immunol. 41, 2503-2508 (2011)

2. Maurer, M. \& von Stebut, E. Macrophage inflammatory protein-1. Int J Biochem Cell Bio. 36, 1882-1886 (2004)

3. Carr, M. W., Roth, S. J., Luther, E., Rose, S. S. \& Springer, T. A. Monocyte chemoattractant protein 1 acts as a T-lymphocyte chemoattractant. Proc Natl Acad Sci USA 91, 3652-3656 (1994).

4. Turner, J. R. Intestinal mucosal barrier function in health and disease. Nat Rev Immunol. 9, 799-809 (2009).

5. Zhu, P., Goh, Y. Y., Chin, H. F. A., Kersten, S. \& Tan, N. S. Angiopoietin-like 4: a decade of research. Biosci Rep. 32, 211-219 (2012).

6. Zhu, P. et al. Angiopoietin-like 4 protein elevates the prosurvival intracellular $\mathrm{O}_{2}(-): \mathrm{H}_{2} \mathrm{O}_{2}$ ratio and confers anoikis resistance to tumors. Cancer Cell. 19, 401-415 (2011).

7. Tan, M. J., Teo, Z., Sng, M. K., Zhu, P. \& Tan, N. S. Emerging roles of angiopoietin-like 4 in human cancer. Mol Cancer Res. 10, 677-688 (2012)

8. Li, L., Chow, V. T. K. \& Tan, N. S. Targeting vascular leakage in lung inflammation. Oncotarget 6, 19338-19339 (2015).

9. Chong, H. C. et al. Angiopoietin-like 4 stimulates STAT3-mediated iNOS expression and enhances angiogenesis to accelerate wound healing in diabetic mice. Mol Ther. 22, 1593-1604 (2014).

10. Lichtenstein, L. et al. Angptl4 protects against severe proinflammatory effects of saturated fat by inhibiting fatty acid uptake into mesenteric lymph node macrophages. Cell Metab. 12, 580-592 (2010).

11. Georgiadi, A. et al. Overexpression of angiopoietin-like protein 4 protects against atherosclerosis development. Arterioscler Thromb Vasc Biol. 33, 1529-1537 (2013).

12. Aryal, B. et al. ANGPTL4 deficiency in haematopoietic cells promotes monocyte expansion and atherosclerosis progression. Nature communications 7, 12313 (2016)

13. Hermann, L. M. et al. Angiopoietin-like-4 is a potential angiogenic mediator in arthritis. Clin Immunol. 115, 93-101 (2005).

14. Li, L. et al. Angiopoietin-like 4 Increases Pulmonary Tissue Leakiness and Damage during Influenza Pneumonia. Cell Reports 10, 654-663 (2015).

15. Tjeerdema, N. et al. Inflammation increases plasma angiopoietin-like protein 4 in patients with the metabolic syndrome and type 2 diabetes. BMJ Open Diabetes Res Care. 2(1), e000034 (2014).

16. Costello, E. K., Stagaman, K., Dethlefsen, L., Bohannan, B. J. \& Relman, D. A. The application of ecological theory toward an understanding of the human microbiome. Science 336, 1255-1262 (2012).

17. Nicholson, J. K. et al. Host-gut microbiota metabolic interactions. Science 336, 1262-1267 (2012).

18. Hooper, L. V. \& Macpherson, A. J. Immune adaptations that maintain homeostasis with the intestinal microbiota. Nat Rev Immunol 10, 159-169 (2010)

19. Marchesi, J. R. et al. The gut microbiota and host health: a new clinical frontier. Gut 65, 330-339 (2016).

20. Chaplin, A., Parra, P., Laraichi, S., Serra, F. \& Palou, A. Calcium supplementation modulates gut microbiota in a prebiotic manner in dietary obese mice. Mol Nutr Food Res. 60, 468-480 (2016).

21. Backhed, F. et al. The gut microbiota as an environmental factor that regulates fat storage. Proc Natl Acad Sci USA 101, 15718-15723 (2004).

22. Camp, J. G., Jazwa, A. L., Trent, C. M. \& Rawls, J. F. Intronic cis-regulatory modules mediate tissue-specific and microbial control of angptl4/fiaf transcription. PLoS genetics 8, e1002585 (2012).

23. Guo, L. et al. Role of Angptl4 in vascular permeability and inflammation. Inflamm Res. 63, 13-22 (2014).

24. Joe, Y. et al. Tristetraprolin mediates anti-inflammatory effects of carbon monoxide on lipopolysaccharide-induced acute lung injury. Am J Pathol. 185, 2867-2874 (2015).

25. Kratochvill, F. et al. Tristetraprolin Limits Inflammatory Cytokine Production in Tumor-Associated Macrophages in an mRNA Decay-Independent Manner. Cancer Res. 75, 3054-3064 (2015).

26. Brennan, C. M. \& Steitz, J. A. HuR and mRNA stability. Cell Mol Life Sci. 58, 266-277 (2001).

27. Fan, J. et al. Chemokine transcripts as targets of the RNA-binding protein HuR in human airway epithelium. J Immunol. 186, 2482-2494 (2011).

28. Brooks, S. A. \& Blackshear, P. J. Tristetraprolin (TTP): interactions with mRNA and proteins, and current thoughts on mechanisms of action. Biochim Biophys Acta. 1829, 666-679 (2013).

29. Lee, H. H. et al. Tristetraprolin down-regulates IL-17 through mRNA destabilization. FEBS Lett. 586, 41-46 (2012).

30. Gaba, A. et al. Cutting edge: IL-10-mediated tristetraprolin induction is part of a feedback loop that controls macrophage STAT3 activation and cytokine production. J Immunol. 189, 2089-2093 (2012) 
31. Goh, Y. Y. et al. Angiopoietin-like 4 interacts with matrix proteins to modulate wound healing. J Biol Chem. 285, 32999-33009 (2010).

32. Xie, F. et al. Identification of a Potent Inhibitor of CREB-Mediated Gene Transcription with Efficacious in Vivo Anticancer Activity. J Med Chem 58, 5075-5087 (2015).

33. Shah, S., Mostafa, M. M., McWhae, A., Traves, S. L. \& Newton, R. Negative Feed-forward Control of Tumor Necrosis Factor (TNF) by Tristetraprolin (ZFP36) Is Limited by the Mitogen-activated Protein Kinase Phosphatase, Dual-specificity Phosphatase 1 (DUSP1): implications for regulation by glucocorticoids. J Biol Chem. 291, 110-125 (2016).

34. Smoak, K. \& Cidlowski, J. A. Glucocorticoids regulate tristetraprolin synthesis and posttranscriptionally regulate tumor necrosis factor alpha inflammatory signaling. Mol Cell Biol. 26, 9126-9135 (2006).

35. Kuo, T. et al. Repression of glucocorticoid-stimulated angiopoietin-like 4 gene transcription by insulin. J Lipid Res. 55, 919-928 (2014).

36. Koliwad, S. K. et al. Angiopoietin-like 4 (ANGPTL4, fasting-induced adipose factor) is a direct glucocorticoid receptor target and participates in glucocorticoid-regulated triglyceride metabolism. J Biol Chem. 284, 25593-25601 (2009).

37. Korecka, A. et al. ANGPTL4 expression induced by butyrate and rosiglitazone in human intestinal epithelial cells utilizes independent pathways. Am J Physiol Gastrointest Liver Physiol. 304, G1025-1037 (2013).

38. Roquilly, A. et al. Hydrocortisone prevents immunosuppression by interleukin-10+ natural killer cells after trauma-hemorrhage. Crit Care Med. 42, e752-761 (2014).

39. Petito, V. et al. Direct effect of infliximab on intestinal mucosa sustains mucosal healing: exploring new mechanisms of action. Dig Liver Dis. 48, 391-398 (2015).

40. Lin, L. et al. Celastrol Ameliorates Ulcerative Colitis-Related Colorectal Cancer in Mice via Suppressing Inflammatory Responses and Epithelial-Mesenchymal Transition. Front Pharmacol. 6, 320 (2015).

41. Goh, Y. Y. et al. Angiopoietin-like 4 interacts with integrins betal and beta5 to modulate keratinocyte migration. Am J Pathol. 177, 2791-2803 (2010).

42. Katsha, A. et al. Aurora kinase A promotes inflammation and tumorigenesis in mice and human gastric neoplasia. Gastroenterology. 145, 1312-1322. e1311 (2013).

43. Fallahi, M., Amelio, A. L., Cleveland, J. L. \& Rounbehler, R. J. CREB targets define the gene expression signature of malignancies having reduced levels of the tumor suppressor tristetraprolin. PloS one. 9, el15517 (2014)

44. Carballo, E., Lai, W. S. \& Blackshear, P. J. Feedback inhibition of macrophage tumor necrosis factor-alpha production by tristetraprolin. Science. 281, 1001-1005 (1998)

45. Ogilvie, R. L. et al. Tristetraprolin mediates interferon-gamma mRNA decay. J Biol Chem. 284, 11216-11223 (2009).

46. Tudor, C. et al. The p38 MAPK pathway inhibits tristetraprolin-directed decay of interleukin-10 and pro-inflammatory mediator mRNAs in murine macrophages. FEBS Lett. 583, 1933-1938 (2009).

47. Xavier, R. J. \& Podolsky, D. K. Unravelling the pathogenesis of inflammatory bowel disease. Nature. 448, 427-434 (2007).

48. Jiang, W. et al. Dysbiosis gut microbiota associated with inflammation and impaired mucosal immune function in intestine of humans with non-alcoholic fatty liver disease. Sci Rep. 5, 8096 (2015).

49. Gkouskou, K. K., Deligianni, C., Tsatsanis, C. \& Eliopoulos, A. G. The gut microbiota in mouse models of inflammatory bowel disease. Front. Cell. Infect. Microbiol. 4, 28 (2014).

50. Bloom, S. M. et al. Commensal Bacteroides species induce colitis in host-genotype-specific fashion in a mouse model of inflammatory bowel disease. Cell Host Microbe. 9, 390-403 (2011).

51. Serada, S. et al. Serum leucine-rich alpha-2 glycoprotein is a disease activity biomarker in ulcerative colitis. Inflamm Bowel Dis. 18, 2169-2179 (2012).

52. Kharbanda, A. B., Rai, A. J., Cosme, Y., Liu, K. \& Dayan, P. S. Novel serum and urine markers for pediatric appendicitis. Acad Emerg Med. 19, 56-62 (2012).

53. Hiller, F., Besselt, K., Deubel, S., Brigelius-Flohe, R. \& Kipp, A. P. GPx2 Induction Is Mediated Through STAT Transcription Factors During Acute Colitis. Inflamm Bowel Dis. 21, 2078-2089 (2015).

54. Lam, C. R. et al. TAK1 regulates SCF expression to modulate PKBalpha activity that protects keratinocytes from ROS-induced apoptosis. Cell Death Differ. 18, 1120-1129 (2011).

55. Olsen, J. et al. Diagnosis of ulcerative colitis before onset of inflammation by multivariate modeling of genome-wide gene expression data. Inflamm Bowel Dis. 15, 1032-1038 (2009).

56. Tan, C. K. et al. SMAD3 deficiency promotes inflammatory aortic aneurysms in angiotensin II-infused mice via activation of iNOS. J Am Heart Assoc. 2, e000269 (2013).

57. Wang, X. et al. Early controlled release of peroxisome proliferator-activated receptor beta/delta agonist GW501516 improves diabetic wound healing through redox modulation of wound microenvironment. J Control Release. 197, 138-147 (2015).

\section{Acknowledgements}

All work in this manuscript was supported by Ministry of Education, Singapore Tier 2 (MOE2014-T2-1-012) and NTU iFood Research Grant (WBS M4081459.080) to NST. TP is a recipient of the NTU-KI Scholarship; EHPT and JSKC are recipients of the Nanyang President's Graduate Scholarship. We wish to acknowledge Dr. Han Chung CHONG and Ming Jie TAN from Denova Sciences Pte Ltd for their assistance in microarray analysis and imaging, and Dr. Abdul Rashid Bin Mohammad Muzaki of the NTU-SBS Flow Facility for his assistance in FACS analysis.

\section{Author Contributions}

N.S.T. and T.P. designed, organized and interpreted the data, and wrote this manuscript. T.P., M.K.S., E.H.P.T., D.S.L.C., Y.L., J.W.K.W., J.S.K.C. and M.M.K.L. performed the experiments. Z.T., C.K.T., P.C.Z. and V.A. assisted in data analyses. N.S.T. supervised the entire study.

\section{Additional Information}

Supplementary information accompanies this paper at http://www.nature.com/srep

Competing Interests: The authors declare no competing financial interests.

How to cite this article: Phua, T. et al. Angiopoietin-like 4 Mediates Colonic Inflammation by Regulating Chemokine Transcript Stability via Tristetraprolin. Sci. Rep. 7, 44351; doi: 10.1038/srep44351 (2017).

Publisher's note: Springer Nature remains neutral with regard to jurisdictional claims in published maps and institutional affiliations. 
(c) (i) This work is licensed under a Creative Commons Attribution 4.0 International License. The images c) or other third party material in this article are included in the article's Creative Commons license, unless indicated otherwise in the credit line; if the material is not included under the Creative Commons license, users will need to obtain permission from the license holder to reproduce the material. To view a copy of this license, visit http://creativecommons.org/licenses/by/4.0/

(C) The Author(s) 2017 


\section{SUPPLEMENTAL INFORMATION}

\section{Angiopoietin-like 4 Mediates Colonic Inflammation by Regulating Chemokine Transcript Stability via Tristetraprolin.}

Terri PHUA ${ }^{1,2}$, Ming Keat $\mathrm{SNG}^{1,3}$, Eddie Han Pin $\mathrm{TAN}^{1}$, Dickson Shao Liang CHEE ${ }^{1}$, Yinliang $\mathrm{LI}^{1}$, Jonathan Wei Kiat WEE ${ }^{1}$, Ziqiang $\mathrm{TEO}^{1}$, Jeremy Soon Kiat $\mathrm{CHAN}^{1}$, Maegan Miang Kee LIM ${ }^{1}$, Chek Kun TAN ${ }^{3}$, Pengcheng ZHU ${ }^{1}$, Velmurugesan ARULAMPALAM ${ }^{2}$, Nguan Soon TAN ${ }^{1,3,4,5^{*}}$

${ }^{1}$ School of Biological Sciences, Nanyang Technological University, 60 Nanyang Drive, Singapore 637551

${ }^{2}$ Department of Microbiology, Tumor and Cell Biology, Karolinska Institutet, Nobels väg 16, Stockholm 17177, Sweden

${ }^{3}$ Lee Kong Chian School of Medicine, Nanyang Technological University, 50 Nanyang Drive, Singapore 639798

${ }^{4}$ Institute of Molecular Cell Biology, 61 Biopolis Drive, Proteos, Agency for Science Technology \& Research, Singapore 138673

${ }^{5}$ KK Research Centre, KK Women's and Children Hospital, 100 Bukit Timah Road, Singapore 229899

Correspondence to NST: nstan@ ntu.edu.sg; Tel: +65 6316 2941; Fax: +65 67913856 . 
Supplementary Table S1. Disease Activity Index (DAI) Score.

\begin{tabular}{cccc}
\hline Score & Weight Loss & Stool Consistency & Visible Blood in Feces \\
\hline $\mathbf{0}$ & $0 \%$ & None & None \\
$\mathbf{1}$ & $1-5 \%$ & & Slight Bleeding \\
$\mathbf{2}$ & $5-10 \%$ & Loose Stool & \\
$\mathbf{3}$ & $10-20 \%$ & & Extensive Bleeding \\
\hline
\end{tabular}

Supplementary Table S2. Endpoint Macroscopic/Disease Severity Score.

\begin{tabular}{ccccc}
\hline Score & Rectal Bleeding & Rectal Prolapse & Stool Consistency & Blood \\
\hline $\mathbf{0}$ & None & None & Normal & Normal \\
$\mathbf{1}$ & Red & Signs of prolapse & Soft & Red \\
$\mathbf{2}$ & Dark Red & Clear prolapse & Very Soft & Dark Red \\
$\mathbf{3}$ & Gross Bleeding & Extensive Prolapse & Diarrhea & Black \\
\hline
\end{tabular}

Supplementary Table S3. Histological Scoring for Degree of Epithelial Damage and

Inflammatory Infiltration.

\begin{tabular}{|c|c|c|c|}
\hline Score & Inflammation Severity & Crypt Damage & Ulceration \\
\hline 0 & Rare inflammatory cells in the lamina propria & Intact crypts & absence of ulcer \\
\hline 1 & $\begin{array}{c}\text { Increased numbers of granulocytes in the } \\
\text { lamina propria }\end{array}$ & Loss of the basal one-third & $<2$ foci of ulcerations \\
\hline 2 & $\begin{array}{l}\text { Confluence of inflammatory cells extending } \\
\text { into the submucosa }\end{array}$ & Loss of the basal two-thirds & $<4$ foci of ulcerations \\
\hline 3 & $\begin{array}{l}\text { Transmural extension of the inflammatory } \\
\text { infiltrate }\end{array}$ & Loss of entire crypts & $\begin{array}{l}\text { confluent or } \\
\text { extensive ulceration }\end{array}$ \\
\hline 4 & - & $\begin{array}{l}\text { Change of epithelial surface } \\
\text { with erosion }\end{array}$ & - \\
\hline 5 & - & Confluent erosion & - \\
\hline
\end{tabular}


Supplementary Table S4. List of Differentially Expressed Genes and their respective expression values in association with Gastrointestinal Diseases.

\begin{tabular}{|c|c|c|c|c|c|}
\hline $\begin{array}{l}\text { Gene } \\
\text { Symbol }\end{array}$ & Vehicle & SA ANGPTL4 ${ }^{+/+}$ & SA ANGPTL4 ${ }^{-/-}$ & DSS ANGPTL4 ${ }^{+/+}$ & DSS ANGPTL4 ${ }^{-/-}$ \\
\hline RETNLB & -26.6188 & -1.25321 & 4.64282 & -5.39947 & 10.1949 \\
\hline SIRT6 & 1.58671 & -1.30123 & -5.54798 & 1.60477 & -1.20498 \\
\hline IL17A & 1.12074 & -1.11832 & -1.05405 & -2.055 & -1.7773 \\
\hline DRD4 & 1.05236 & -1.5765 & -1.24962 & -1.32943 & -1.56154 \\
\hline PON1 & 1.97514 & -1.10116 & -1.09437 & -1.09882 & -2.90767 \\
\hline FCER1A & 1.34889 & -1.07422 & 1.1706 & -1.21276 & -1.70433 \\
\hline NFE2L2 & -2.7691 & -1.35215 & -1.38063 & -3.7374 & -1.14798 \\
\hline B3GNT6 & -2.10196 & -1.1442 & -1.17655 & -3.1278 & -1.60115 \\
\hline NR5A2 & -1.5738 & -2.0245 & -1.36879 & -1.56857 & -1.40889 \\
\hline PROC & -1.25318 & -1.64026 & -1.35534 & -1.62156 & -1.60218 \\
\hline TRPV1 & -1.37549 & -1.16311 & -1.6602 & -1.98386 & -1.36789 \\
\hline CEBPE & -1.19974 & -1.05284 & -1.12961 & -2.07957 & -1.43371 \\
\hline SERPINE1 & -1.58514 & -1.41746 & -1.25299 & -2.10557 & -1.033 \\
\hline CORT & -1.60273 & -1.012 & -1.09575 & -2.01711 & -1.25602 \\
\hline MST1 & -1.75233 & -1.05904 & -1.10075 & -1.80311 & -1.07072 \\
\hline IL25 & -1.44409 & -1.04595 & -1.31383 & -1.68468 & -1.36804 \\
\hline FUT7 & -1.43967 & -1.08146 & -1.41597 & -1.40863 & -1.20905 \\
\hline AGR2 & -1.31688 & -1.4246 & -1.33041 & -1.28983 & -1.07755 \\
\hline IL13 & -1.17519 & -1.06607 & -1.11831 & -1.40748 & -1.19772 \\
\hline IL23A & -1.22906 & -1.08807 & -1.1708 & -1.09771 & -1.12181 \\
\hline HPGD & -1.02934 & -1.1409 & -1.88522 & -1.08238 & -1.73129 \\
\hline PDE4C & -1.16904 & 1.02033 & -1.2904 & -1.76682 & -1.44068 \\
\hline JAK3 & -1.20285 & 1.20139 & -1.38017 & -1.28041 & -1.38338 \\
\hline CRLF2 & -1.00953 & 1.04459 & -1.32036 & -1.12094 & -1.16585 \\
\hline APOA4 & -1.04016 & 1.30988 & -1.11326 & -1.42492 & -1.2794 \\
\hline FKBP1A & -2.19355 & -1.54415 & -1.42833 & -3.03289 & 1.02716 \\
\hline ID3 & -1.38537 & -1.40079 & -2.20764 & -3.39579 & 1.23223 \\
\hline HSF1 & -1.497 & -2.26297 & -2.13899 & -1.46727 & 1.03598 \\
\hline PTPN11 & -1.51045 & -1.70983 & -2.33757 & -1.19645 & 1.20427 \\
\hline NFKB1 & -1.1295 & -2.02506 & -1.8885 & -1.02364 & 1.25495 \\
\hline MTOR & -2.00182 & -2.26367 & -1.091 & -1.5921 & 1.26378 \\
\hline VEGFA & -2.17882 & -1.97848 & -1.38317 & -1.38931 & 2.03393 \\
\hline PTGS1 & -1.97047 & -2.0465 & -1.04333 & -1.08905 & 1.63136 \\
\hline MERTK & -1.85714 & -1.71701 & -1.57332 & -1.01989 & 1.58254 \\
\hline JAK1 & -1.82148 & -1.4327 & -1.75069 & -1.1433 & 1.66069 \\
\hline RAC1 & -1.93969 & -1.27498 & -1.0857 & -1.77756 & 1.13242 \\
\hline
\end{tabular}




\begin{tabular}{|c|c|c|c|c|c|}
\hline РPP3СA & -1.71001 & -1.72684 & -1.39265 & -1.30021 & 1.03945 \\
\hline TRAF3IP2 & -1.46481 & -1.51732 & -1.75997 & -1.591 & 1.1241 \\
\hline Map3k7 & -1.54306 & -1.46731 & -1.6109 & -1.24003 & 1.14156 \\
\hline LTB & -1.29939 & -1.67018 & -1.19537 & -1.75935 & 1.02987 \\
\hline ITGB3 & -1.4162 & -1.8556 & -1.17229 & -1.46268 & 1.24162 \\
\hline NLRX1 & -1.27339 & -1.30732 & -1.34163 & -1.63348 & 1.09034 \\
\hline CD40 & -1.41569 & -1.13296 & -1.38161 & -1.34019 & 1.04531 \\
\hline SELE & -1.30216 & -1.02828 & -1.1178 & -1.45899 & 1.0242 \\
\hline GCNT3 & -1.7527 & -1.08844 & -1.54661 & -1.02564 & 1.39333 \\
\hline HSP90B1 & -1.45019 & -1.01837 & -1.28478 & -1.07022 & 1.31761 \\
\hline STAP2 & -1.04524 & -1.82845 & -1.04225 & -1.01008 & 1.15859 \\
\hline TLR9 & -1.11351 & -1.64595 & -1.14105 & -1.02772 & 1.39376 \\
\hline ANXA1 & -2.2405 & 1.02251 & -2.68851 & -2.00896 & 1.84666 \\
\hline S1PR4 & -1.79524 & 1.0563 & -1.00132 & -1.68558 & 1.04522 \\
\hline SOD1 & -1.89037 & 1.60346 & -1.31383 & -1.38129 & 1.06448 \\
\hline PROCR & -1.16328 & 1.14168 & -1.69279 & -1.25741 & 1.05706 \\
\hline IL27RA & -1.19545 & 1.06105 & -1.13638 & -1.23417 & 1.06253 \\
\hline CD44 & -1.1668 & 1.1498 & -1.14438 & -1.27485 & 1.06489 \\
\hline STAT5A & -1.1369 & 1.22899 & -1.21412 & -1.11786 & 1.1354 \\
\hline NKX2-3 & -1.2083 & 1.07732 & -1.03985 & -1.09584 & 1.34038 \\
\hline RAG1 & -2.00338 & -1.48789 & 2.14076 & -2.07728 & -1.06656 \\
\hline ALOX12 & -1.47344 & -1.67266 & 1.6672 & -2.24655 & -1.49486 \\
\hline CYP11B1 & -1.60169 & -1.54329 & 1.16741 & -2.14192 & -1.13875 \\
\hline SOCS1 & -1.38137 & -1.26633 & 1.28739 & -2.55959 & -1.19905 \\
\hline CALCA & -1.36846 & -1.08872 & 1.32864 & -2.02409 & -1.60363 \\
\hline TBX21 & -1.69255 & -1.10917 & 1.44342 & -1.6745 & -1.27891 \\
\hline PLG & -1.25363 & -1.04103 & 1.26468 & -1.61668 & -1.19262 \\
\hline IL21 & -1.25166 & -1.04097 & 1.44289 & -1.79289 & -1.08921 \\
\hline PGLYRP2 & -1.29207 & -1.02418 & 1.49765 & -1.38411 & -1.04748 \\
\hline DRD2 & -1.28427 & -1.33718 & 1.11135 & -1.3641 & -1.22718 \\
\hline BID & -1.08705 & -1.3221 & 1.10094 & -1.13724 & -1.07721 \\
\hline PDE4A & -1.11174 & -1.10246 & 1.09427 & -1.22686 & -1.06803 \\
\hline DRD3 & -2.09993 & -1.45169 & 1.08058 & -2.07108 & 1.14421 \\
\hline ALOX5 & -1.75646 & -1.75883 & 1.21825 & -1.69646 & 1.04325 \\
\hline RXRA & -1.41533 & -1.99749 & 1.505 & -1.41533 & 1.06201 \\
\hline POR & -1.56913 & -2.089 & 1.2376 & -1.03348 & 1.50171 \\
\hline CRP & -1.59623 & -1.34974 & 1.49501 & -1.73895 & 1.05988 \\
\hline BATF & -1.61161 & -1.16806 & 1.60481 & -1.7622 & 1.08132 \\
\hline TKTL1 & -1.39718 & -1.24981 & 1.60785 & -1.82859 & 1.00825 \\
\hline ADORA2A & -1.57238 & -1.25024 & 1.69087 & -1.44974 & 1.0619 \\
\hline EBI3 & -1.48586 & -1.12135 & 1.48807 & -1.45179 & 1.25108 \\
\hline
\end{tabular}




\begin{tabular}{|c|c|c|c|c|c|}
\hline IL2 & -1.22685 & -1.00257 & 1.75146 & -1.39365 & 1.13582 \\
\hline NOD2 & -1.38484 & -1.44373 & 1.36766 & -1.38311 & 1.07226 \\
\hline $\mathrm{CDH} 1$ & -1.55877 & -1.77433 & 1.40625 & -1.20528 & 1.3836 \\
\hline NFKBID & -1.68562 & -1.54046 & 1.52507 & -1.36841 & 1.37383 \\
\hline NOS3 & -1.47938 & -1.53841 & 1.57869 & -1.29937 & 1.28667 \\
\hline HTR7 & -1.39511 & -1.40025 & 1.58485 & -1.22475 & 1.6514 \\
\hline RUNX3 & -1.13678 & -1.35953 & 1.43174 & -1.08961 & 1.37892 \\
\hline TREX1 & -1.16251 & -1.00275 & 1.22233 & -1.26651 & 1.11976 \\
\hline RASGRP4 & -1.50867 & -1.11397 & 1.06686 & -1.09975 & 1.58407 \\
\hline IL1R1 & -1.63231 & -1.07927 & 1.17933 & -1.192 & 2.81466 \\
\hline NFIL3 & -2.60854 & -2.43028 & 1.46341 & -1.39627 & 3.44323 \\
\hline CKM & -2.21212 & 1.46795 & 1.84386 & -3.88946 & -1.28993 \\
\hline MEP1A & -1.18545 & 1.26504 & 1.62529 & -1.46591 & -2.99943 \\
\hline BSN & -1.65624 & 1.13292 & 1.29964 & -1.86133 & -1.05234 \\
\hline DRD5 & -1.27351 & 1.0402 & 1.47924 & -1.76156 & -1.1581 \\
\hline MPO & -1.14963 & 1.24997 & 1.13302 & -1.67043 & -1.31289 \\
\hline IFNG & -1.0258 & 1.09285 & 1.19431 & -1.12442 & -1.07517 \\
\hline CD40LG & -1.11526 & 1.1636 & 1.47449 & -1.28332 & -1.16061 \\
\hline PGLYRP3 & -1.20701 & 1.11443 & 1.59727 & -1.29865 & -1.11216 \\
\hline ESR2 & -1.21616 & 1.0409 & 1.61505 & -1.21272 & -1.02877 \\
\hline CSF2 & -1.06841 & 1.49153 & 1.8323 & -1.14348 & -1.20635 \\
\hline TRPM8 & -1.06825 & 1.75878 & 1.14576 & -1.12163 & -1.39738 \\
\hline IL4 & -1.14174 & 1.10666 & 2.22296 & -1.43256 & -1.19431 \\
\hline CYP11A1 & -1.22238 & 1.25683 & 2.51989 & -1.47142 & -1.06899 \\
\hline ALB & 1.19747 & 1.04177 & 1.18117 & -1.06722 & -1.63433 \\
\hline IL24 & 1.01303 & 1.5918 & 1.67301 & -1.06248 & -1.12344 \\
\hline MME & 1.02132 & 1.41249 & 2.52821 & -1.30279 & -1.28318 \\
\hline PPARG & -1.94427 & 1.51837 & 1.24371 & -1.7641 & 1.19287 \\
\hline CALCB & -1.89205 & 1.41566 & 1.70846 & -2.05855 & 1.11461 \\
\hline IL12A & -1.60121 & 1.01815 & 2.12528 & -1.93616 & 1.09617 \\
\hline IL22 & -1.17297 & 1.00585 & 1.51543 & -1.63395 & 1.97593 \\
\hline MMP7 & -1.65341 & 1.23486 & 1.65503 & -1.25154 & 1.23674 \\
\hline SIGIRR & -1.18122 & 1.05917 & 1.33369 & -1.22699 & 1.08792 \\
\hline IL23R & -1.29396 & 1.13352 & 1.48381 & -1.05412 & 1.35519 \\
\hline WAS & -1.0857 & 1.09616 & 1.59823 & -1.11237 & 1.45503 \\
\hline ITGB7 & -1.24647 & 1.10803 & 1.92843 & -1.06249 & 1.23373 \\
\hline $\mathrm{Ccl} 2$ & -1.66945 & 1.2296 & 2.056 & -1.10835 & 1.90177 \\
\hline $\mathrm{Ccl} 2$ & -1.66945 & 1.2296 & 2.056 & -1.10835 & 1.90177 \\
\hline CR2 & -1.08288 & 1.95694 & 1.57529 & -1.23283 & 1.69063 \\
\hline PGLYRP4 & -1.74188 & 1.53334 & 2.39859 & -1.5389 & 1.15389 \\
\hline PDCD1 & -1.68359 & 1.24356 & 2.84018 & -1.64444 & 1.21547 \\
\hline
\end{tabular}




\begin{tabular}{|c|c|c|c|c|c|}
\hline IL10 & -1.46245 & 1.14761 & 2.88133 & -1.18316 & 1.54387 \\
\hline STAT6 & -1.30016 & -2.57358 & -1.84793 & 1.12096 & 1.37862 \\
\hline ITGA1 & -1.40833 & -1.4551 & -2.3596 & 1.1914 & 1.48545 \\
\hline IRF3 & -1.06951 & -1.28953 & -1.75937 & 1.01439 & 1.22236 \\
\hline AHR & -1.04713 & -1.38696 & -1.58564 & 1.15732 & 1.14741 \\
\hline STX2 & -1.08302 & -1.71137 & -1.69874 & 1.12409 & 1.64993 \\
\hline DDX58 & -1.38222 & -1.16978 & -1.15213 & 1.01099 & 1.36929 \\
\hline ATG16L1 & -1.06252 & -1.35452 & -1.10566 & 1.06644 & 1.28045 \\
\hline PDE4B & -1.481 & -1.30477 & -1.15957 & 1.08216 & 1.7176 \\
\hline PIK3CD & -1.19695 & -1.36932 & -1.21022 & 1.01904 & 1.8112 \\
\hline ICAM1 & -1.12922 & -1.41927 & -1.18683 & 1.19976 & 2.0483 \\
\hline IL1RN & -1.10143 & -1.38788 & -1.03174 & 1.7474 & 1.69256 \\
\hline C3 & -1.68126 & -1.97542 & -2.49761 & 2.31674 & 2.7554 \\
\hline TNFRSF1A & -1.09713 & -1.66177 & -1.80691 & 1.92805 & 2.5234 \\
\hline IL6ST & -1.08629 & -1.31998 & -1.18184 & 2.11966 & 2.62532 \\
\hline STAT1 & -1.03316 & -1.22273 & -1.21251 & 1.90765 & 3.25507 \\
\hline CD274 & -1.04028 & -1.99381 & -1.10074 & 2.18924 & 3.89993 \\
\hline PTGS2 & -1.97925 & -1.3019 & -1.54534 & 1.58974 & 4.45818 \\
\hline NLRP6 & 1.17997 & -1.78366 & -2.15082 & 1.3403 & -1.09509 \\
\hline RAC3 & 1.16945 & -1.09155 & -1.31408 & 1.03373 & -1.16945 \\
\hline FAAH & 1.31787 & -1.07043 & -1.47256 & 1.29516 & -1.05414 \\
\hline SMAD7 & 1.61498 & -1.29873 & -1.02701 & 1.1959 & -1.35974 \\
\hline PHB & 2.28424 & -1.07405 & -1.70313 & 1.49525 & -1.45846 \\
\hline TPMT & 2.15391 & -1.01194 & -1.96843 & 1.72489 & -1.17363 \\
\hline PYCARD & 2.92642 & -1.10603 & -1.82476 & 3.20785 & -1.3755 \\
\hline EDN1 & 1.29646 & -2.51659 & -2.00331 & 1.71405 & 1.48887 \\
\hline XBP1 & 1.94201 & -2.65388 & -1.46594 & 1.74532 & 1.12364 \\
\hline GPX2 & 2.12319 & -2.67529 & -1.42045 & 1.02146 & 2.02904 \\
\hline TRAF2 & 1.18797 & -1.64956 & -1.77769 & 1.10533 & 1.13745 \\
\hline GPX1 & 1.28542 & -1.56572 & -1.75153 & 1.40496 & 1.2074 \\
\hline ABCC4 & 1.2224 & -1.19904 & -1.42674 & 1.52685 & 1.4991 \\
\hline RPTOR & 1.03235 & -2.00154 & -1.22256 & 1.36818 & 1.31137 \\
\hline TGFB1 & 1.33674 & -1.74343 & -1.10928 & 1.44106 & 1.70122 \\
\hline AKT1 & 2.31563 & -1.78267 & -1.29387 & 1.61879 & 1.05119 \\
\hline LGALS4 & 1.76501 & -1.48001 & -1.0853 & 1.62791 & 1.01459 \\
\hline STAT5B & 1.62087 & -1.18976 & -1.4971 & 1.78372 & 1.21549 \\
\hline LTBR & 1.90908 & -1.35937 & -1.27052 & 1.9874 & 1.17344 \\
\hline GTPBP1 & 2.08531 & -1.053 & -1.25931 & 2.38207 & 1.00968 \\
\hline TFF3 & 1.54439 & -1.16569 & -1.34886 & 2.37195 & 1.59218 \\
\hline COL1A1 & 1.42936 & -1.89733 & -1.61133 & 1.91599 & 1.92556 \\
\hline STAT3 & 1.24422 & -1.49867 & -1.6117 & 2.07328 & 2.01191 \\
\hline
\end{tabular}




\begin{tabular}{|c|c|c|c|c|c|}
\hline TGFBR2 & 1.55764 & -1.73728 & -1.04745 & 2.22275 & 2.09301 \\
\hline COL1A2 & 1.54003 & -1.29655 & -1.47134 & 2.26325 & 2.42547 \\
\hline CFB & 1.22193 & -1.75355 & -1.0137 & 2.85426 & 2.60359 \\
\hline HPGDS & 1.29833 & -1.29193 & -1.19181 & 3.53669 & 2.42167 \\
\hline FAS & 1.02361 & -1.04475 & -1.56447 & 2.99063 & 3.01426 \\
\hline CSF1R & 2.14256 & -1.35453 & -1.52388 & 3.51295 & 2.35429 \\
\hline MYD88 & 2.3735 & -2.78234 & -1.44625 & 3.7431 & 1.91635 \\
\hline IL10RB & 2.64618 & -2.85961 & -1.09453 & 4.06753 & 1.30899 \\
\hline KRAS & -1.0818 & 3.44966 & -3.25919 & 1.50156 & 1.17326 \\
\hline TLR2 & -1.23749 & 1.08891 & -1.10471 & 1.14635 & 1.57774 \\
\hline DDIT3 & -1.13516 & 1.52218 & -1.08529 & 1.1533 & 1.31871 \\
\hline TLR1 & -1.07657 & 1.60355 & -1.16999 & 1.22882 & 1.47395 \\
\hline NOD1 & -1.37543 & 1.55792 & -1.02677 & 1.12507 & 1.75135 \\
\hline B2M & -1.01352 & 1.28174 & -1.24845 & 1.89814 & 1.74976 \\
\hline HSF2 & -1.03352 & 2.49994 & -1.14628 & 1.53628 & 1.31122 \\
\hline TLR4 & -1.05335 & 1.81016 & -2.31818 & 1.5363 & 1.98114 \\
\hline CCL11 & -1.58055 & 1.73723 & -1.40988 & 1.38563 & 2.74515 \\
\hline CD47 & -1.28137 & 3.82436 & -1.25429 & 2.60584 & 3.05922 \\
\hline ATM & 1.04759 & 1.01685 & -1.99078 & 1.49357 & 1.14115 \\
\hline TNFAIP8 & 1.26517 & 1.04402 & -1.93902 & 1.659 & 1.50925 \\
\hline TNFAIP3 & 1.29807 & 1.14978 & -1.88753 & 1.63354 & 1.9782 \\
\hline SMAD3 & 1.02789 & 1.0188 & -1.13196 & 1.40517 & 1.71746 \\
\hline F2RL1 & 1.02679 & 1.69078 & -1.18163 & 1.69445 & 1.64769 \\
\hline ITGA4 & 1.61911 & 1.32338 & -2.40836 & 2.68561 & 1.71311 \\
\hline$A X L$ & 1.51246 & 1.20566 & -1.80288 & 2.48566 & 1.70236 \\
\hline JAK2 & 1.35359 & 1.35254 & -1.61361 & 2.55728 & 1.89524 \\
\hline PROS1 & 1.43364 & 1.26306 & -1.85696 & 2.73403 & 2.53528 \\
\hline IL7 & 1.20265 & 1.76105 & -1.00331 & 2.49812 & 1.00466 \\
\hline CASP1 & 1.63288 & 2.53568 & -2.15564 & 2.0891 & 1.18771 \\
\hline ATP11C & 1.07495 & 2.92215 & -1.58812 & 2.46417 & 1.70011 \\
\hline CR1L & 1.65646 & 3.71986 & -2.00429 & 2.71468 & 1.23355 \\
\hline CKB & 2.17095 & 1.1382 & -4.07999 & 3.04446 & 1.17899 \\
\hline CRBN & 2.00387 & 1.73112 & -3.1418 & 2.80311 & 1.05185 \\
\hline IL18 & 2.48296 & 1.24017 & -2.76151 & 3.95895 & 1.49787 \\
\hline ENTPD1 & 2.49697 & 1.44078 & -3.29715 & 3.97206 & 2.28863 \\
\hline LY96 & 1.73233 & 1.36609 & -2.34586 & 4.86187 & 1.66274 \\
\hline ID2 & 3.41051 & 2.32825 & -1.31236 & 3.82715 & 1.39019 \\
\hline VDR & -1.16707 & -2.39178 & 1.50576 & 1.04406 & -1.15479 \\
\hline FERMT1 & -1.03852 & -1.25715 & 1.15029 & 1.2737 & -1.19526 \\
\hline ERN2 & 1.17839 & -2.01899 & 1.18919 & 1.8399 & -1.03618 \\
\hline IL22RA2 & 1.41036 & 1.35181 & 1.52681 & 1.38398 & -1.23789 \\
\hline
\end{tabular}




\begin{tabular}{|c|c|c|c|c|c|}
\hline IFNB1 & 1.31756 & 1.01373 & 1.97103 & 1.33854 & -1.14159 \\
\hline PIP & 1.15697 & 1.43735 & 2.11715 & 1.03299 & -1.10134 \\
\hline NRG4 & 1.1667 & 2.00222 & 2.2079 & 1.20857 & -1.51717 \\
\hline AKT2 & -1.4754 & -2.8049 & 1.17837 & 1.06033 & 1.80109 \\
\hline CEACAM1 & -1.23378 & -1.40504 & 1.09442 & 1.10696 & 1.27983 \\
\hline PIK3R1 & -1.20881 & -1.03504 & 1.351 & 1.07032 & 1.20972 \\
\hline PLS1 & -1.2801 & -1.15342 & 1.30416 & 1.10937 & 1.55576 \\
\hline F10 & -1.3612 & -1.02499 & 1.34582 & 1.10198 & 1.51719 \\
\hline IL2RA & -1.1629 & -1.20627 & 1.67881 & 1.23773 & 1.56122 \\
\hline PIK3CG & -1.00657 & -1.70243 & 1.38037 & 1.5127 & 1.54673 \\
\hline CTLA4 & -1.44901 & -1.27595 & 2.04779 & 1.06545 & 1.963 \\
\hline DMBT1 & -1.63303 & -1.23155 & 2.16134 & 1.94448 & 3.30078 \\
\hline IL6 & -1.2921 & -1.20194 & 3.77616 & 1.23142 & 2.70156 \\
\hline TNFRSF11B & -1.20234 & 1.13038 & 1.21879 & 1.05709 & 1.29307 \\
\hline NR3C1 & -1.21579 & 1.23663 & 1.00196 & 1.1518 & 1.34884 \\
\hline TLR3 & -1.18448 & 1.20297 & 1.11373 & 1.31416 & 1.30573 \\
\hline РРP3СС & -1.33787 & 1.26169 & 1.33565 & 1.3808 & 1.33199 \\
\hline ALOX15 & -1.03146 & 1.24802 & 1.35281 & 1.58157 & 1.11082 \\
\hline IL12B & -1.12705 & 1.0792 & 1.79723 & 1.16114 & 1.27572 \\
\hline CD80 & -1.07047 & 1.1673 & 1.79182 & 1.16824 & 1.36869 \\
\hline ADAMTS12 & -1.52372 & 1.08295 & 1.54216 & 1.05914 & 1.79677 \\
\hline IL13RA2 & -1.02982 & 1.31415 & 2.24483 & 1.29808 & 1.95026 \\
\hline CXCL10 & -1.23354 & 1.43813 & 1.79403 & 1.48444 & 4.08562 \\
\hline NLRP3 & -1.19481 & 1.35447 & 2.30694 & 2.3255 & 2.83283 \\
\hline TYK2 & 1.24185 & -1.353 & 1.06705 & 1.26122 & 1.11137 \\
\hline KSR1 & 1.14312 & -1.4129 & 1.17775 & 1.55673 & 1.70665 \\
\hline NCF1 & 1.10163 & -1.08527 & 1.1492 & 1.73512 & 2.03481 \\
\hline CD69 & 1.00405 & -1.07282 & 1.57186 & 1.68661 & 2.55396 \\
\hline GNAI2 & 1.68937 & -1.43281 & 1.13918 & 2.09806 & 1.35224 \\
\hline GNAI2 & 1.68937 & -1.43281 & 1.13918 & 2.09806 & 1.35224 \\
\hline TNFRSF14 & 1.55123 & -1.09474 & 1.02392 & 2.03333 & 1.78266 \\
\hline RELA & 2.11036 & -1.34066 & 1.52934 & 1.90412 & 1.11825 \\
\hline GUCY2C & 2.07913 & -1.53966 & 1.53528 & 2.26251 & 1.00212 \\
\hline RORC & 1.70711 & -1.28754 & 1.04349 & 2.94119 & 1.25254 \\
\hline FGFR2 & 1.28582 & -1.16658 & 1.00974 & 3.01045 & 1.67707 \\
\hline ADM & 1.69298 & -1.10755 & 1.52419 & 2.49359 & 3.00715 \\
\hline CCR5 & 1.0213 & -1.04346 & 1.30101 & 3.47081 & 3.6519 \\
\hline ITGB8 & 1.10243 & 1.44749 & 1.17829 & 1.18371 & 1.61066 \\
\hline SPP1 & 1.19356 & 1.26573 & 1.00382 & 2.12677 & 1.28588 \\
\hline MMP9 & 1.29794 & 1.08506 & 1.0841 & 2.15277 & 1.39096 \\
\hline PDE4D & 1.53377 & 1.3404 & 1.2399 & 2.63356 & 1.15824 \\
\hline
\end{tabular}




\begin{tabular}{|c|c|c|c|c|c|}
\hline РPРЗСВ & 1.55466 & 1.80873 & 1.10411 & 2.19592 & 1.04349 \\
\hline IL10RA & 1.1248 & 1.07662 & 1.54193 & 1.7779 & 2.41555 \\
\hline HSD11B1 & 1.20162 & 1.1637 & 1.63652 & 1.7798 & 2.88012 \\
\hline NCF4 & 1.29552 & 1.30862 & 1.2629 & 2.04625 & 2.53647 \\
\hline STAT4 & 1.31313 & 1.04788 & 1.93287 & 2.08256 & 2.64075 \\
\hline CXCL3 & 1.14316 & 1.22465 & 1.99026 & 2.38905 & 2.5253 \\
\hline NR1H4 & 1.12738 & 1.33626 & 1.15467 & 2.67755 & 2.05239 \\
\hline S1PR1 & 1.38752 & 1.47645 & 1.02028 & 3.09947 & 2.22284 \\
\hline PTPN22 & 1.52082 & 1.18684 & 1.51551 & 3.54386 & 1.90429 \\
\hline CYLD & 1.22994 & 3.13735 & 1.10372 & 2.35591 & 1.79937 \\
\hline $\mathrm{MC} 2 \mathrm{R}$ & -1.18598 & 1.82783 & 4.86515 & 1.13151 & -1.13127 \\
\hline RAG2 & -1.06394 & 1.19881 & 4.98807 & 1.13033 & 1.0224 \\
\hline SLPI & -2.12001 & -1.70489 & 1.64073 & 1.49557 & 5.57916 \\
\hline CXCL5 & -1.23289 & -1.12782 & 1.27556 & 1.44991 & 6.04236 \\
\hline TNF & -1.71711 & 1.07873 & 1.93168 & 1.62914 & 6.46805 \\
\hline SELP & -1.27778 & 1.02891 & 2.10372 & 1.9938 & 5.77376 \\
\hline $\mathrm{CFH}$ & -1.15477 & 1.50003 & -2.25178 & 3.5769 & 5.69614 \\
\hline TREM1 & -1.44136 & 1.15221 & 1.05275 & 4.79722 & 5.511 \\
\hline HGF & 1.17141 & 1.34558 & -1.22794 & 3.07991 & 4.4313 \\
\hline EREG & 1.48455 & 1.54003 & 1.10689 & 3.71949 & 4.70649 \\
\hline Retnla & -1.00485 & 1.75934 & -1.41336 & 7.45647 & 3.99548 \\
\hline CXCL2 & -1.04405 & 1.49699 & 1.72401 & 6.70428 & 9.86742 \\
\hline CCR2 & 1.19437 & 2.44401 & 2.00809 & 7.26107 & 9.03643 \\
\hline NFKBIA & 3.49968 & -1.02344 & 1.01129 & 7.3258 & 2.67856 \\
\hline SOCS3 & -1.8566 & -1.51803 & 1.91034 & 1.81516 & 11.9349 \\
\hline LTF & -1.7211 & -1.04272 & 1.86351 & 4.26719 & 13.3968 \\
\hline LBP & -1.6013 & -1.37887 & -1.81481 & 3.6804 & 17.7245 \\
\hline NOS2 & -1.65396 & -1.38193 & 1.33736 & 3.74231 & 18.9493 \\
\hline S100A8 & -1.26127 & 1.06691 & 1.46931 & 6.76554 & 17.1899 \\
\hline LCN2 & 1.31501 & -1.39367 & -1.23284 & 18.5878 & 12.1224 \\
\hline MUC1 & 4.22658 & 1.68306 & 1.1663 & 40.5054 & 6.60718 \\
\hline IL1B & -1.22156 & 1.34929 & 1.77778 & 23.5381 & 36.4379 \\
\hline IGHM & 60.7957 & -1.16806 & -1.71707 & 3.82298 & -11.3306 \\
\hline
\end{tabular}


Supplementary Table S5. List of Differentially Expressed Genes and their respective expression values in association with Gastrointestinal Diseases in comparision between murine and human samples.

\begin{tabular}{|c|c|c|c|c|}
\hline $\begin{array}{l}\text { Gene } \\
\text { Symbol }\end{array}$ & ANGPTL $^{+/+}$DSS & $\begin{array}{r}\text { Ulcerative Colitis } \\
\text { (non-inflamed) }\end{array}$ & ANGPTL4 ${ }^{-/-}$DSS & $\begin{array}{r}\text { Ulcerative Colitis } \\
\text { (inflamed) }\end{array}$ \\
\hline CKB & 2.17095 & 3.04446 & 1.17899 & -1.88829 \\
\hline FAS & 1.02361 & 2.99063 & 3.01426 & -1.54032 \\
\hline RORC & 1.70711 & 2.94119 & 1.25254 & -1.51346 \\
\hline GUCY2C & 2.07913 & 2.26251 & 1.00212 & -1.44301 \\
\hline PIK3R1 & -1.20881 & 1.07032 & 1.20972 & -1.4242 \\
\hline EDN1 & 1.29646 & 1.71405 & 1.48887 & -1.38378 \\
\hline ERN2 & 1.17839 & 1.8399 & -1.03618 & -1.23377 \\
\hline FAAH & 1.31787 & 1.29516 & -1.05414 & -1.22826 \\
\hline EREG & 1.48455 & 3.71949 & 4.70649 & -1.12482 \\
\hline VDR & -1.16707 & 1.04406 & -1.15479 & -1.08755 \\
\hline ESR2 & -1.21616 & -1.21272 & -1.02877 & 1.09874 \\
\hline ITGB3 & -1.4162 & -1.46268 & 1.24162 & 1.15755 \\
\hline JAK1 & -1.82148 & -1.1433 & 1.66069 & 1.17046 \\
\hline IL17A & 1.12074 & -2.055 & -1.7773 & 1.20362 \\
\hline WAS & -1.0857 & -1.11237 & 1.45503 & 1.22069 \\
\hline $\mathrm{BID}$ & -1.08705 & -1.13724 & -1.07721 & 1.23141 \\
\hline VEGFA & -2.17882 & -1.38931 & 2.03393 & 1.25006 \\
\hline ALOX5 & -1.75646 & -1.69646 & 1.04325 & 1.2522 \\
\hline CD44 & -1.1668 & -1.27485 & 1.06489 & 1.25621 \\
\hline IL1R1 & -1.63231 & -1.192 & 2.81466 & 1.25621 \\
\hline CD40 & -1.41569 & -1.34019 & 1.04531 & 1.31529 \\
\hline ANXA1 & -2.2405 & -2.00896 & 1.84666 & 1.31543 \\
\hline HSP90B1 & -1.45019 & -1.07022 & 1.31761 & 1.34164 \\
\hline ITGB7 & -1.24647 & -1.06249 & 1.23373 & 1.36925 \\
\hline SOD1 & -1.89037 & -1.38129 & 1.06448 & 1.46972 \\
\hline MERTK & -1.85714 & -1.01989 & 1.58254 & 1.50204 \\
\hline SOCS1 & -1.38137 & -2.55959 & -1.19905 & 1.55949 \\
\hline PROCR & -1.16328 & -1.25741 & 1.05706 & 1.59804 \\
\hline CCL2 & -1.66945 & -1.10835 & 1.90177 & 2.21867 \\
\hline
\end{tabular}


Supplementary Table S6. Fold change of chemokine expression in iCECs when treated with various pro- and anti-inflammatory stimuli.

\begin{tabular}{|c|c|c|c|c|c|c|c|c|c|c|c|c|c|c|c|}
\hline & \multirow{2}{*}{$\begin{array}{l}\text { Treatments } \\
\text { Genes }\end{array}$} & \multicolumn{2}{|c|}{ Veh } & \multicolumn{2}{|c|}{ DSS } & \multicolumn{2}{|c|}{ SA } & \multicolumn{2}{|c|}{ IL-1 $\beta$} & \multicolumn{2}{|c|}{ TNF- $\alpha$} & \multicolumn{2}{|c|}{$\mathrm{HC}$} & \multicolumn{2}{|c|}{$\mathrm{NaBu}$} \\
\hline & & Mean & SEM & Mean & SEM & Mean & SEM & Mean & SEM & Mean & SEM & Mean & SEM & Mean & SEM \\
\hline \multirow{6}{*}{ iCEC $_{\mathrm{ctrl}}$} & CXCL10 & 1 & 0.02 & 5.92 & 0.06 & 1.46 & 0.01 & 2.73 & 0.01 & 102.68 & 1.88 & 0.2 & 0.01 & 0.02 & 0 \\
\hline & CCL11 & 1 & 0 & 2.37 & 0.01 & 1.72 & 0.04 & 2.23 & 0.04 & 4.78 & 0.02 & 0.6 & 0.12 & 0.45 & 0.03 \\
\hline & IL-1 $\beta$ & 1 & 0.06 & 0.94 & 0.07 & 0.83 & 0.06 & & & 2.73 & 0.69 & 0.37 & 0.05 & 0.8 & 0.05 \\
\hline & IL-10 & 1 & 0 & 0.31 & 0.03 & 0.56 & 0.02 & 0.46 & 0.02 & 0.16 & 0.02 & 1.57 & 0 & 1.18 & 0.03 \\
\hline & ANGPTL4 & 1 & 0.01 & 0.68 & 0.02 & 0.81 & 0.01 & 0.41 & 0.09 & 0.31 & 0 & 1.48 & 0.03 & 2.13 & 0.01 \\
\hline & TTP & 1 & 0.01 & 0.52 & 0.01 & 0.45 & 0 & 0.72 & 0.09 & 0.06 & 0 & 5.34 & 0.01 & 1.71 & 0.01 \\
\hline \multirow{3}{*}{$\mathrm{iCEC}_{\text {TTP }}$} & CXCL10 & 1 & 0.01 & 1.37 & 0.02 & 1.3 & 0.04 & 1.92 & 0.02 & 59.88 & 0.31 & 0.3 & 0.01 & 0.56 & 0.02 \\
\hline & CCL2 & 1 & 0.05 & 1.35 & 0.06 & 0.68 & 0.07 & 2.78 & 0.03 & 2.43 & 0.1 & 0.96 & 0.02 & 0.62 & 0.06 \\
\hline & CCL11 & 1 & 0.05 & 2.13 & 0.09 & 2.23 & 0.04 & 2.8 & 0.06 & 3.97 & 0.11 & 0.78 & 0.13 & 1.03 & 0.01 \\
\hline \multirow{7}{*}{ iCEC $_{\text {ANGPTL4 }}$} & CXCL10 & 1 & 0.01 & 9.61 & 0.06 & 5.8 & 0.09 & 9.38 & 0.04 & 156.34 & 5.39 & 0.29 & 0.02 & 0.68 & 0.02 \\
\hline & $\mathrm{CCL} 2$ & 1 & 0 & 1.3 & 0 & 0.97 & 0 & 2.67 & 0.01 & 2.37 & 0.01 & 0.66 & 0 & 0.61 & 0 \\
\hline & CCL11 & 1 & 0.01 & 2.11 & 0.02 & 1.16 & 0.02 & 2.99 & 0.02 & 4.9 & 0.03 & 0.35 & 0.02 & 0.78 & 0.02 \\
\hline & IL-1 $\beta$ & 1 & 0.01 & 1.44 & 0.11 & 2.21 & 0.28 & & & 3 & 0.71 & 0.35 & 0.08 & 0.09 & 0.01 \\
\hline & IL-10 & 1 & 0.01 & 0.76 & 0.02 & 0.92 & 0.01 & 0.97 & 0.02 & 0.57 & 0.05 & 2.17 & 0.01 & 4.54 & 0.03 \\
\hline & IL-17 & 1 & 0.03 & 0.64 & 0.05 & 1.07 & 0.04 & 0.65 & 0.02 & 0.36 & 0.05 & 2.55 & 0.02 & 4.48 & 0.01 \\
\hline & TTP & 1 & 0.01 & 0.57 & 0.01 & 0.65 & 0 & 0.9 & 0.02 & 0.44 & 0 & 1.23 & 0 & 1.37 & 0.15 \\
\hline
\end{tabular}


Supplementary Table S7. Fold change of chemokine concentration in iCECs when treated with various pro- and anti-inflammatory stimuli.

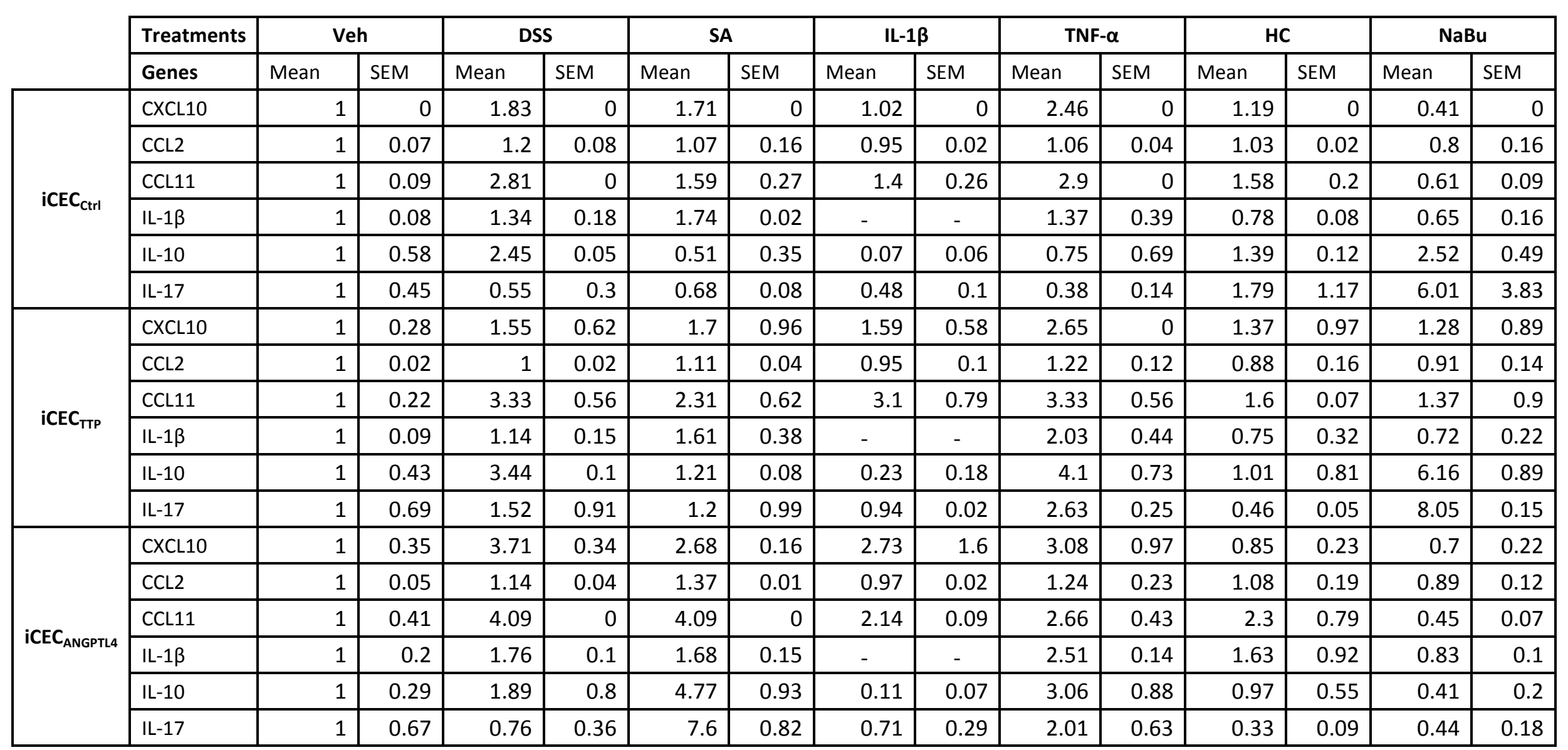


Supplementary Table S8. Sequences of human ON-TARGETplus SMARTPool siRNA targets for ANGPTL4-, CREB-, RELA- or TTP-knockdown.

\begin{tabular}{|c|c|c|}
\hline Gene Symbol & Species & siRNA smartpool target sequences (5' to $3^{\prime}$ ) \\
\hline \multirow[b]{4}{*}{ ANGPTL4 } & \multirow[b]{4}{*}{ Human } & GAUGGAGGCUGGACAGUAA \\
\hline & & CCACUUGGGACCAGGAUCA \\
\hline & & GAAAGAGGCUGCCCGAGAU \\
\hline & & GGCAGAAGCUUAAGAAGGG \\
\hline \multirow[b]{4}{*}{ CREB1 } & \multirow[b]{4}{*}{ Human } & GAGAGAGGUCCGUCUAAUG \\
\hline & & UAGUACAGCUGCCCAAUGG \\
\hline & & CAACUCCAAUUUACCAAAC \\
\hline & & GCCCAGCCAUCAGUUAUUC \\
\hline \multirow[b]{4}{*}{ RELA } & \multirow[b]{4}{*}{ Human } & GGAUUGAGGAGAAACGUAA \\
\hline & & CCCACGAGCUUGUAGGAAA \\
\hline & & GGCUAUAACUCGCCUAGUG \\
\hline & & CCACACAACUGAGCCCAUG \\
\hline \multirow[b]{4}{*}{ TTP } & \multirow[b]{4}{*}{ Human } & GCAAGUAGCCAAAGCCGUU \\
\hline & & CCCAAAUACAAGACGGAAC \\
\hline & & GGACAGUAAUCAAGUAAUC \\
\hline & & GCAUAUUUAAGGGAGGCAA \\
\hline
\end{tabular}


Supplementary Table S9. Sequences of real-time PCR primers used in this study.

\begin{tabular}{|c|c|c|c|c|}
\hline $\begin{array}{l}\text { Gene } \\
\text { Symbol }\end{array}$ & Species & Gene ID & $\begin{array}{l}\text { Forward primer sequence } \\
\left.\text { (5' to } 3^{\prime}\right)\end{array}$ & $\begin{array}{l}\text { Reverse primer sequence } \\
\left(5^{\prime} \text { to } 3^{\prime}\right)\end{array}$ \\
\hline $18 \mathrm{~S}$ & $\begin{array}{l}\text { Human/ } \\
\text { Mouse }\end{array}$ & 19791 & GTAACCCGTTGAACCCCATT & CCATCCAATCGGTAGTAGCG \\
\hline ANGPTL4 & Human & 51129 & TGGTTTGGCACCTGCAGCCATTC & TGCTGCCATGGGCTGGATCAAC \\
\hline ANGPTL4 & Mouse & 57875 & CCCCACGCACCTAGACAATG & GCCTCCATCTGAAGTCATCTCA \\
\hline CCL2 & Human & 6347 & CAGCCAGATGCAATCAATGCC & TGGAATCCTGAACCCACTTCT \\
\hline $\mathrm{CCL} 2$ & Mouse & 20296 & TTAAAAACCTGGATCGGAACCAA & GCATTAGCTTCAGATTTACGGGT \\
\hline CCL11 & Human & 6356 & CCCCTTCAGCGACTAGAGAG & TCTTGGGGTCGGCACAGAT \\
\hline CCL11 & Mouse & 20292 & GAATCACCAACAACAGATGCAC & ATCCTGGACCCACTTCTTCTT \\
\hline CREB1 & Human & 1385 & TTAACCATGACCAATGCAGCA & TGGTATGTTTGTACGTCTCCAGA \\
\hline CXCL10 & Human & 3627 & GTGGCATTCAAGGAGTACCTC & TGATGGCCTTCGATTCTGGATT \\
\hline CXCL10 & Mouse & 15945 & CCAAGTGCTGCCGTCATTTTC & GGCTCGCAGGGATGATTTCAA \\
\hline $\mathrm{IL}-1 \beta$ & Human & 3553 & ATGATGGCTTATTACAGTGGCAA & GTCGGAGATTCGTAGCTGGA \\
\hline IL-1 $\beta$ & Mouse & 16176 & GAAATGCCACCTTTTGACAGTG & TGGATGCTCTCATCAGGACAG \\
\hline IL-6 & Mouse & 16193 & TCTATACCACTTCACAAGTCGGA & GAATTGCCATTGCACAACTCTTT \\
\hline IL-10 & Human & 3586 & GACTTTAAGGGTTACCTGGGTTG & TCACATGCGCCTTGATGTCTG \\
\hline IL-10 & Mouse & 16153 & CTTACTGACTGGCATGAGGATCA & GCAGCTCTAGGAGCATGTGG \\
\hline IL-17 & Human & 3605 & TCCCACGAAATCCAGGATGC & GGATGTTCAGGTTGACCATCAC \\
\hline IL-17 & Mouse & 16171 & CGGAGAATTAGTCCCTGTGTTG & GAACAGTCACTTCATACTCCTGG \\
\hline IFN- $\gamma$ & Mouse & 15978 & ACAGCAAGGCGAAAAAGGATG & TGGTGGACCACTCGGATGA \\
\hline $\begin{array}{l}\text { pCREB/pNF- } \\
\text { кB Binding } \\
\text { Site on TTP }\end{array}$ & Human & - & TGCAAGTGGAAAGTCGGAG & TGGAAGTCCGGATTGCTTC \\
\hline $\begin{array}{l}\text { pCREB/pNF- } \\
\text { kB Negative } \\
\text { Control on } \\
\text { TTP }\end{array}$ & Human & - & CCAGACAGAGGGAACTGCAAG & GAGCATGCAGTGTGCACCAG \\
\hline RELA & Human & 5970 & GTGGGGACTACGACCTGAATG & GGGGCACGATTGTCAAAGATG \\
\hline TTP & Human & 7538 & GACTGAGCTATGTCGGACCTT & GAGTTCCGTCTTGTATTTGGGG \\
\hline TTP & Mouse & 22695 & CCGAATCCCTCGGAGGACTT & GAGCCAAAGGTGCAAAACCA \\
\hline TNF- $\alpha$ & Mouse & 21926 & СССТCACACTCAGATCATCTTC & GCTACGACGTGGGCTACAG \\
\hline
\end{tabular}


Supplementary Table S10. Concentrations of kinase inhibitors in the Kinase Inhibitor Array used in this study.

\begin{tabular}{|c|c|c|c|}
\hline Position & Inhibitor & Inhibitor Target & $\begin{array}{l}\text { Concentration } \\
\text { (nM) }\end{array}$ \\
\hline $\mathrm{A} 1$ & DMSO & DMSO & - \\
\hline $\mathrm{A} 2$ & R428 & $A X L$ & 14 \\
\hline A3 & Purvalanol B & CDK & 9 \\
\hline A4 & NVP-BGJ398 & FGFR $1 / 2 / 3$ & 10 \\
\hline A5 & Tofacitinib & JAK3 & 91 \\
\hline A6 & TAK-715 & MAPK, $\mathrm{p} 38 \alpha$ & 30 \\
\hline A7 & PP242 & mTOR & 8 \\
\hline A8 & GDC-0941 & $\mathrm{PI} 3 \mathrm{~K} \alpha / \delta$ & 42 \\
\hline A9 & (R)-BI-2536 & PLK & 5 \\
\hline A10 & GNF-5837 & TRK & 42 \\
\hline A11 & Axitinib & c-Kit, PDGFR $\beta$, VEGFR $1 / 2 / 3$ & 2 \\
\hline A12 & MK-2461 & c-Met, FGFR 1/2/3, PDGFR $\beta$ & 2.5 \\
\hline B1 & $\mathrm{A}-674563 \mathrm{HCl}$ & AKT, CDK, PKA & 16 \\
\hline B2 & ABT-737 & $\mathrm{Bcl} 2$ & 40 \\
\hline B3 & $\mathrm{BMS} 387032 \mathrm{HCl}$ & CDK & 62 \\
\hline B4 & PD-0173074 & FGFR 1 & 25 \\
\hline B5 & $\mathrm{CC}-401 \mathrm{HCl}$ & JNK & 50 \\
\hline B6 & AS703026 & MEK $1 / 2$ & 11 \\
\hline B7 & Rapamycin & mTOR & 1 \\
\hline B8 & IC87114 & PI3Kס & 500 \\
\hline B9 & PF431396 & PYK2 & 31 \\
\hline B10 & GW441756 & TrkA & 2 \\
\hline B11 & Bosutinib & BCR-Abl, Src & 1.2 \\
\hline B12 & Motesanib & c-Kit, PDGFR, VEGFR 1/2/3 & 6 \\
\hline $\mathrm{C} 1$ & MK-2206 2HCl & Akt $1 / 2 / 3$ & 65 \\
\hline $\mathrm{C} 2$ & Imatinib mesylate & BCR-Abl & 600 \\
\hline C3 & PF0477736 & CHK1 & 50 \\
\hline C4 & Quizartinib & FLT3, FMS, cKIT, PDGFR & 4.2 \\
\hline $\mathrm{C} 5$ & Masatinib & $\mathrm{c}-\mathrm{KIT}$ & 800 \\
\hline C6 & $\mathrm{Cl}-1040$ & MEK $1 / 2$ & 17 \\
\hline $\mathrm{C7}$ & XL388 & mTOR & 10 \\
\hline $\mathrm{C} 8$ & PIK-75 & PI3K, p110a & 6 \\
\hline C9 & PLX4032 & $\mathrm{B}-\mathrm{RAF}$ & 31 \\
\hline $\mathrm{C} 10$ & GDC-046 & TYK2 & 13.3 \\
\hline C11 & Cabozantinib & c-MET, Tle2, AXL, Kit & 14.3 \\
\hline $\mathrm{C} 12$ & Ponatinib & BCR-Abl, VEGFR2, FGFR1, Src & 6 \\
\hline D1 & LDK378 & ALK & 0.2 \\
\hline
\end{tabular}




\begin{tabular}{|c|c|c|c|}
\hline D2 & GCl1746 & BTK & 2 \\
\hline D3 & CX-4945 & CK2 & 1 \\
\hline D4 & SB-216763 & GSK-3 & 35 \\
\hline D5 & AMG-47a & Lck & 10 \\
\hline D6 & Selumetinib & MEK1 & 50 \\
\hline D7 & BX795 & PDK1 & 15 \\
\hline D8 & PIK-90 & $\mathrm{PI} 3 \mathrm{~K} \alpha / \beta / \delta$ & 60 \\
\hline D9 & GSK429286A & ROCK & 15 \\
\hline D10 & Tivozanib & VEGFR $1 / 2 / 3$, PDGFR, c-Kit & 1 \\
\hline D11 & Dasatinib & BCR-Abl, Src, c-Kit & 1 \\
\hline D12 & Regorafenib & VEGFR1/2/3, PDGFR $\beta$, KIT, RET, Raf1 & 46 \\
\hline E1 & S-99 & ASK & 5 \\
\hline E2 & PD173955-Analog1 & c-Src & 10 \\
\hline E3 & Gefitinib & EGFR & 30 \\
\hline E4 & Mubritinib & HER2 & 6 \\
\hline E5 & BMS-5 & LIMK & 8 \\
\hline E6 & Crizotinib & C-MET & 24 \\
\hline E7 & GSK2606414 & PERK & 3.2 \\
\hline E8 & TGX221 & $\mathrm{PI} 3 \mathrm{~K}, \mathrm{p} 110 \beta$ & 50 \\
\hline E9 & BI-D1870 & RSK & 31 \\
\hline E10 & JNJ-38158471 & VEGFR2 & 50 \\
\hline E11 & CHIR-258 & $\begin{array}{l}\text { c-KIT, FGFR 1/3, FLT3, FMS, PDGFR, } \\
\text { VEGFR 1-4 }\end{array}$ & 36 \\
\hline E12 & Sorafenib & B-RAF, VEGFR2, Raf-1 & 90 \\
\hline F1 & CYC116 & Aurora A/B & 70 \\
\hline F2 & Alvocidib & CDK $1 / 2 / 4 / 6$ & 40 \\
\hline F3 & Lapatinib & EGFR, HER2 & 11 \\
\hline F4 & LY2784544 & JAK2 & 3 \\
\hline F5 & RWJ-67657 & MAPK (p38MAP) & 200 \\
\hline F6 & PF04217903 & c-MET & 5 \\
\hline F7 & AS-252424 & PI3Ky & 33 \\
\hline F8 & YM201636 HCl & PIKfyve & 33 \\
\hline F9 & PF-04708671 & S6K & 160 \\
\hline $\mathrm{F} 10$ & SAR131675 & VEGFR3 & 45 \\
\hline F11 & E7080 & VEGFR $2 / 3$ & 6 \\
\hline $\mathrm{F} 12$ & SU-6668 & VEGF, PDGFR, FGFR1 & 8 \\
\hline G1 & SNS-314 & Aurora A/B/C & 31 \\
\hline G2 & AT-7519 HCl & CDK 1/2/4/6/9 & 210 \\
\hline G3 & PF-562271 & FAK & 1.5 \\
\hline G4 & Momelotinib & JAK1/2 & 20 \\
\hline G5 & SB202190 & MAPK (p38MAP), p38 $\alpha / \beta$ & 100 \\
\hline G6 & Foretinib & c-MET, VEGFR & 1 \\
\hline G7 & BKM120 & $\mathrm{PI} 3 \mathrm{~K}, \mathrm{p} 110 \alpha / \beta / \mathrm{v} / \delta$ & 20 \\
\hline
\end{tabular}




\begin{tabular}{|l|l|l|r|} 
G8 & SMI-4a & Pim & 21 \\
\hline G9 & R406 & Syk & 41 \\
\hline G10 & Pazopanib & C-KIT, PDGFR1/2/3, FGFR, c-Fms & 146 \\
\hline G11 & Intedanib & VEGFR1/2/3, FGFR1/2/3,PDGFR $\alpha / \beta$ & 100 \\
\hline G12 & Sunitinib malate & C-KIT, PDGFR $\beta$, VEGFR2 & 80 \\
\hline H1 & Tozasertib & Aurora A & 70 \\
\hline H2 & Palbociclib Isethionate & CDK 4/6 & 16 \\
\hline H3 & Brivanib & FGFR1, VEGFR2 & 25 \\
\hline H4 & TG101348 & JAK2 & 3 \\
\hline H5 & SD169 & MAPK (p38MAP) & 4 \\
\hline H6 & MPI0479605 HCl & Mps1, TTK & 4 \\
\hline H7 & GDC-0032 & PI3K $\alpha /$ Y/ & 10 \\
\hline H8 & Bisindoylmaleimide X HCl & PKC & 15 \\
\hline H9 & AMG-Tie2-1 & TIE2 & 1 \\
\hline H10 & Amuvatinib & Fit3, KIT, PDGFR & 81 \\
\hline H11 & Linifanib & Fit1/3, PDGFR $\beta$, VEGFR2, CSF-1R & 66 \\
\hline H12 & Tandutinib & C-KIT, FLT3, PDGFR & 220 \\
\hline
\end{tabular}


Supplementary Table S11. Genotyping PCR primer sequences used in this study.

\begin{tabular}{|l|l|l|l|}
\hline Gene & Species & $\begin{array}{l}\text { Forward primer sequence } \\
\left.\text { (5' to } \mathbf{3}^{\prime}\right)\end{array}$ & $\begin{array}{l}\text { Reverse primer sequence } \\
\left.\text { (5' to } \mathbf{3}^{\prime}\right)\end{array}$ \\
\hline ANGPTL4 & Mouse & GCAAGATCCAGCAATTGTTCCAG & ACCCTTGATGAGAGCCTAGTGAG \\
\hline ANGPTL4 & Mouse & GCAAGATCCAGCAATTGTTCCAG & GGTGCCCCAAGGACCTGAAATGA \\
\hline
\end{tabular}




\section{Supplementary Figures and Legends}
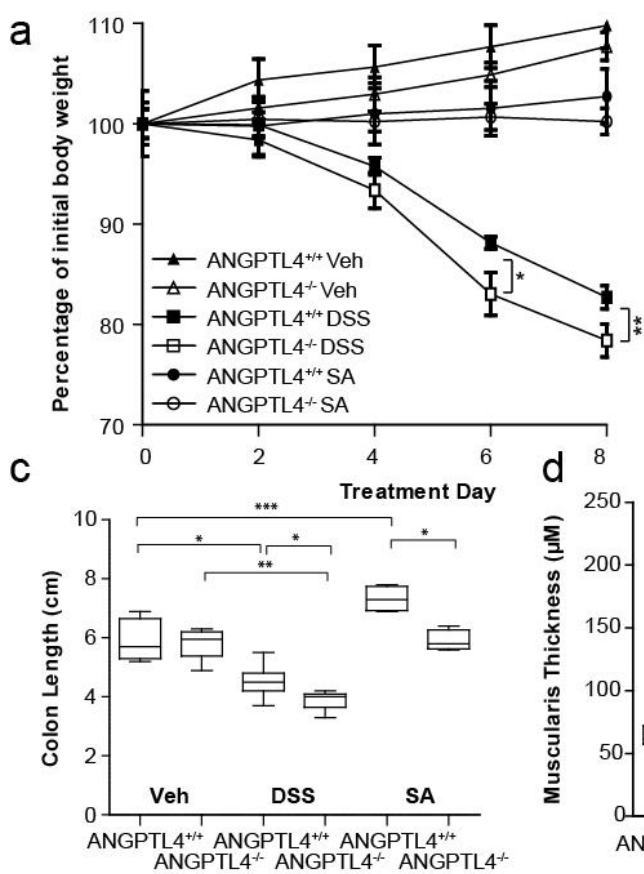

f

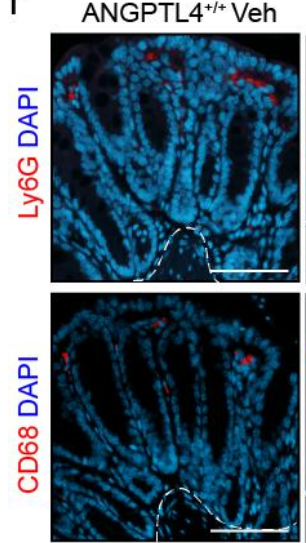

ANGPTL $4^{+/+}$
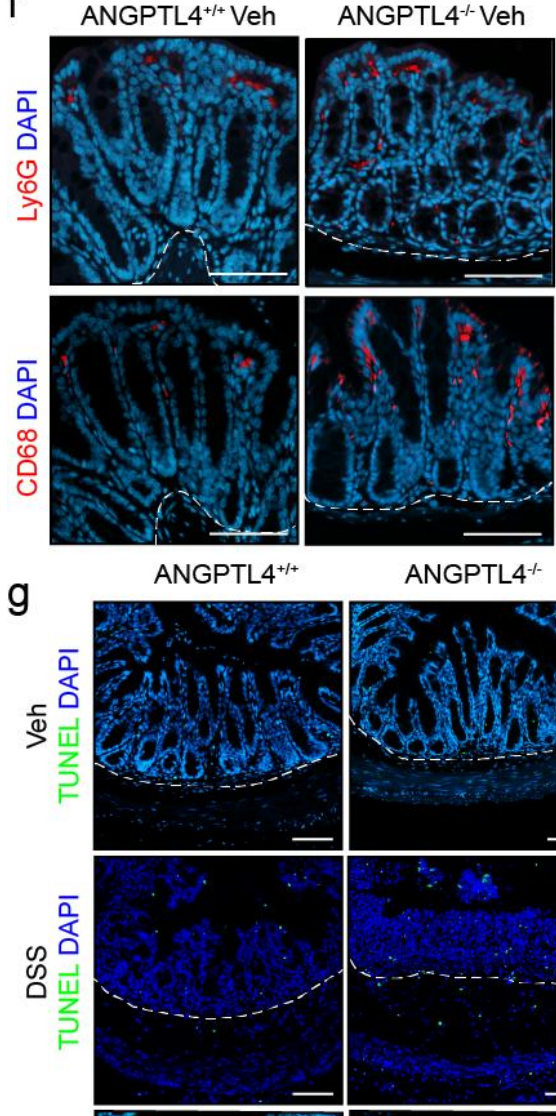

ANGPTL $4^{-1}$
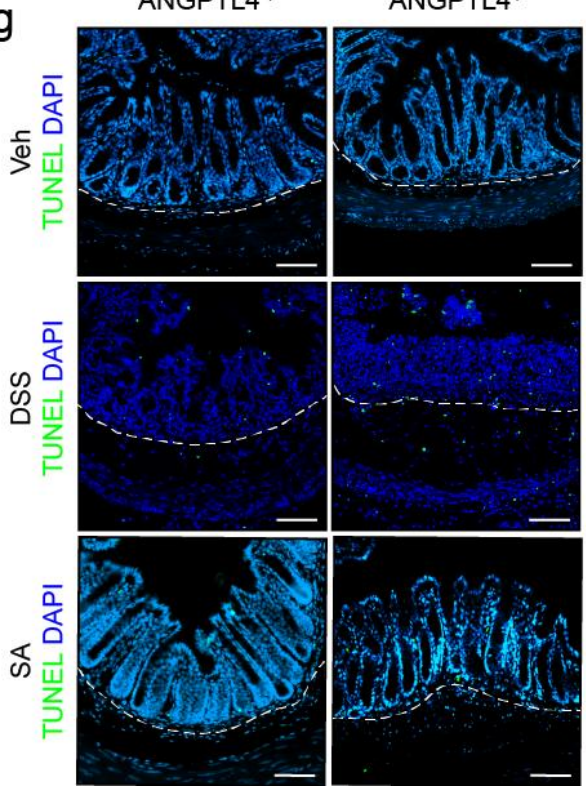

b $\square$ ANGPTL4 ${ }^{+/+}$
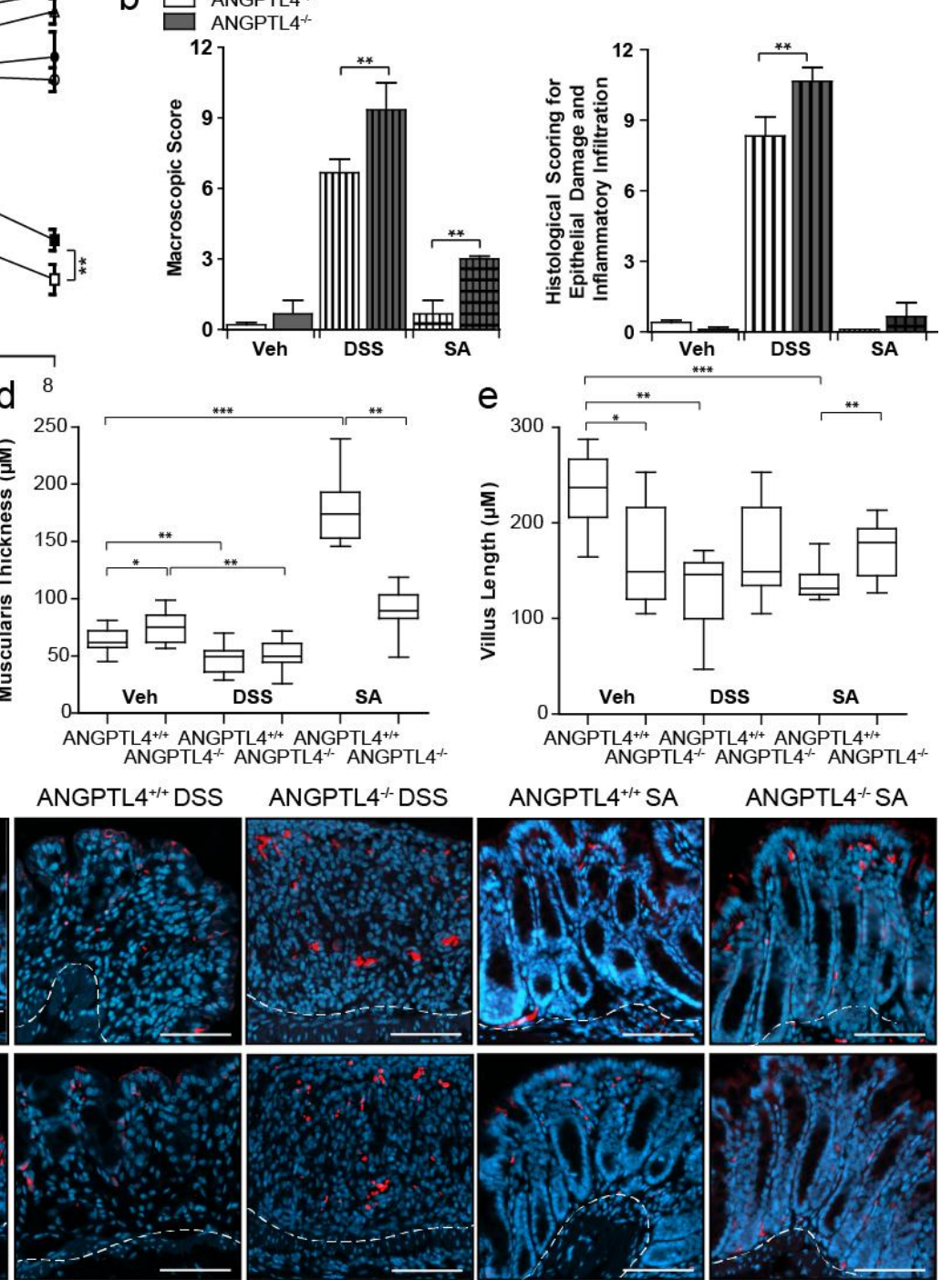

ANGPTL4 ${ }^{-\nsim}$ DSS

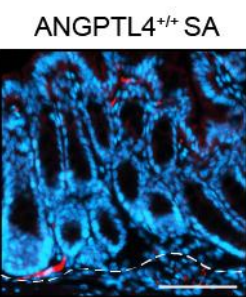

ANGPTL4 ${ }^{--}$SA
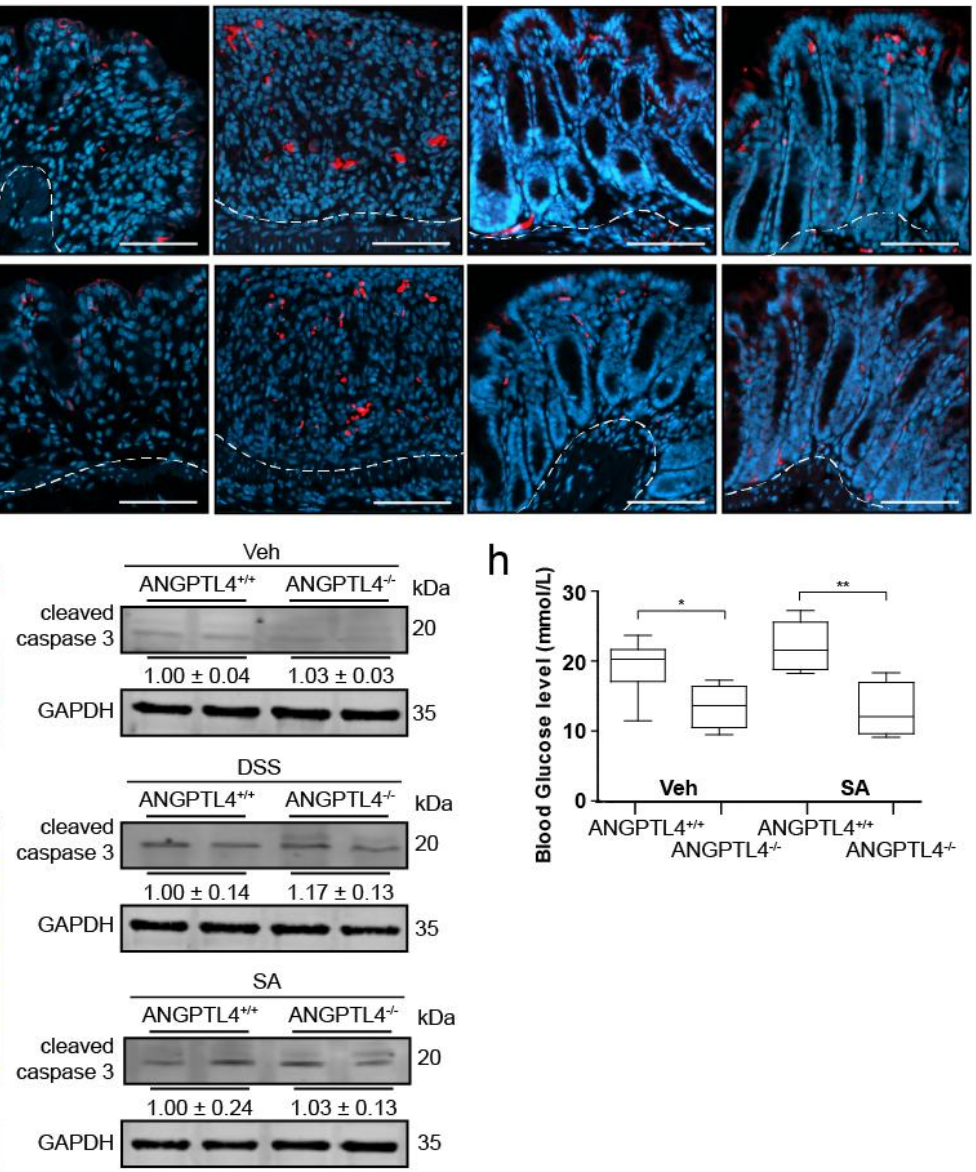

$\mathrm{h}$

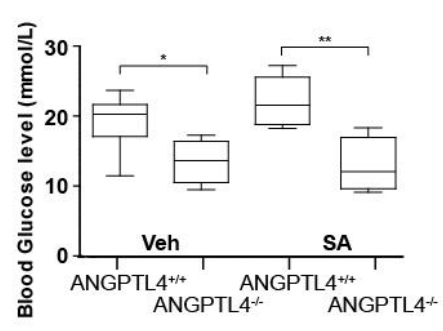




\section{Supplementary Fig. S1. Parameters of colon samples from ANGPTL4 ${ }^{+/+}$and}

\section{ANGPTL4 ${ }^{-/-}$mice given Veh, DSS and SA at 8-day post-treatment.}

(a) Percentage change in body weight of ANGPTL4 $4^{+/+}$and ANGPTL4 $4^{-/-}$mice given Veh, DSS or SA over an 8-day treatment. (b) Macroscopic (left panel) and histological (right panel) scoring for mice at the endpoint; scoring criteria can be found in Supplementary Tables 2-3 respectively. Quantitative measurements of (c) colon length, (d) muscularis thickness and (e) villus length at the 8-day post-treatment experimental endpoint. (f) Immunofluorescence staining for Ly6G (neutrophils; red) and CD68 (macrophages; red) and nuclei (blue) in colon samples from ANGPTL4 ${ }^{+/+}$and ANGPTL4 $4^{-/-}$mice following the indicated treatments. (g) TUNEL staining (left panel) and immunoblot analysis of cleaved caspase 3 (right panel) and for apoptotic cells in colon sections from ANGPTL4 ${ }^{+/+}$and ANGPTL $^{-/-}$mice following the indicated treatment. Scale bar $=100 \mu \mathrm{m}$. (h) Quantitative measurements of plasma blood glucose levels in ANGPTL4 $4^{+/+}$and ANGPTL4 $4^{-/-}$mice after Veh, DSS and SA treatments for 8 days. For the Veh, DSS or SA groups, $n=20$ mice were used in each treatments. The Mann-Whitney U test was used. ${ }^{*} \mathrm{p}<0.05, * * \mathrm{p}<0.01,{ }^{*} * \mathrm{p}<$ 0.001 . 

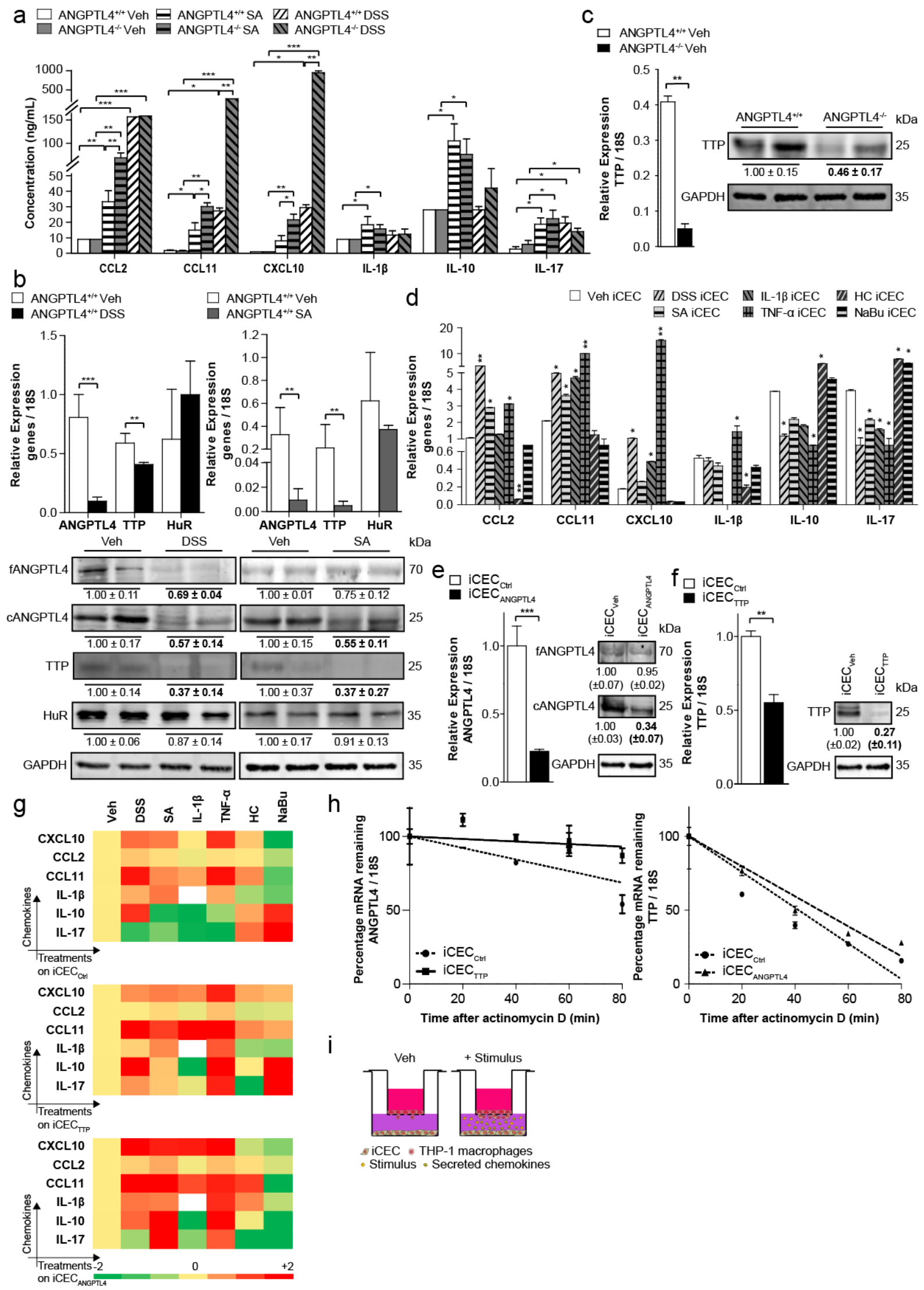


\section{Supplementary Fig. S2. Chemokine expression levels in ANGPTL4 ${ }^{+/+}$and ANGPTL4 ${ }^{-/-}$ mice.}

(a) Relative concentration of the indicated chemokines in colon tissues from ANGPTL $4^{+/+}$ and ANGPTL4 ${ }^{-/-}$mice treated with Veh, DSS or SA. (b) Relative expression (upper panel) and immunoblot analysis (lower panel) of ANGPTL4, TTP and HuR levels in ANGPTL4 ${ }^{+/+}$ littermates for DSS and SA treatments with respect to Veh. (c) Relative mRNA and protein levels of TTP in colon tissues from ANGPTL $4^{+/+}$and ANGPTL4 $4^{-/-}$mice. (d) Relative mRNA expression of the indicated chemokines in iCECs treated with DSS, SA, IL-1 $\beta$, TNF- $\alpha$, HC or $\mathrm{NaBu}$. Relative mRNA (left panel) and protein (right panel) levels accounting for levels of (e) ANGPTL4 and (f) TTP following siRNA knockdown as determined by real-time PCR and immunoblot analysis, respectively. (g) Heatmap showing log-transformed protein concentration of indicated chemokine mRNA transcripts in $\mathrm{iCEC}_{\mathrm{Ctrl}}, \mathrm{iCEC}_{\mathrm{TTP}}$ and iCEC $_{\text {ANGPTL4 }}$ following stimulation with DSS, SA, IL-1 $\beta$, TNF- $\alpha, \mathrm{HC}$ or NaBu. (h) Decay curves of ANGPTL4 and TTP in $\mathrm{iCEC}_{\mathrm{ANGPTL} 4}, \mathrm{iCEC}_{\mathrm{TTP}}$ or $\mathrm{iCEC}_{\mathrm{Ctrl}}$, following actinomycin $\mathrm{D}$ treatment after $\mathrm{NaBu}$ stimulation. (i) Transwell migration assay set-up. siRNA knockdown was performed on iCECs seeded in wells while THP1-derived macrophages were cultured on inserts. The macrophages were later introduced to iCECs and further stimulated with either pro- or anti-inflammatory stimuli. The Mann-Whitney U test was used. ${ }^{*} \mathrm{p}<0.05$, **p $<0.01$, $* * * \mathrm{p}<0.001$. 
a Veh

$\square$ rh-nANGPTL4

$\square$ rh-CANGPTL4

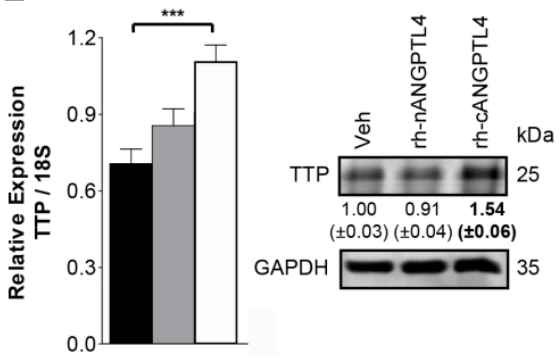

c

- Control

$\square$ rh-CANGPTL4
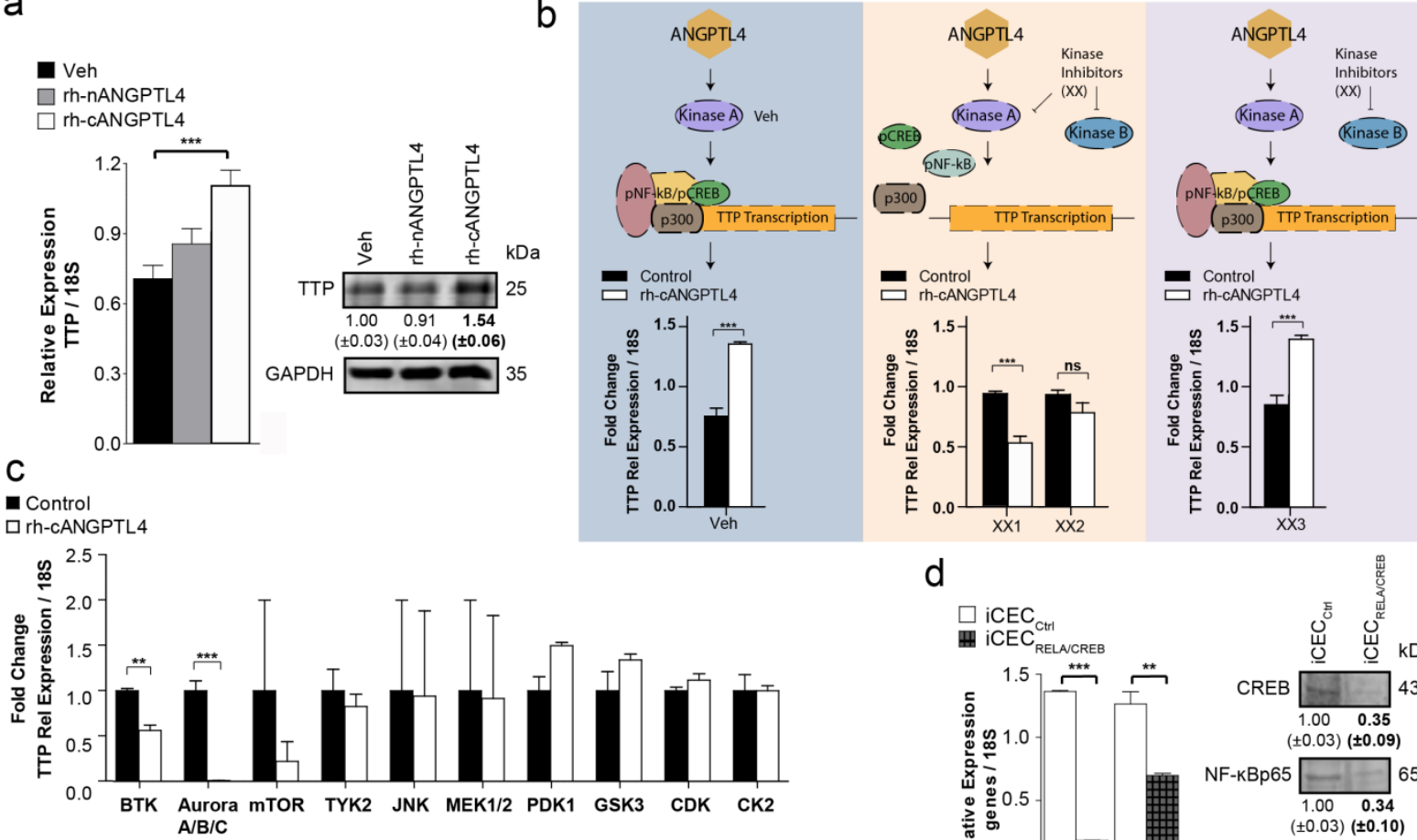

d

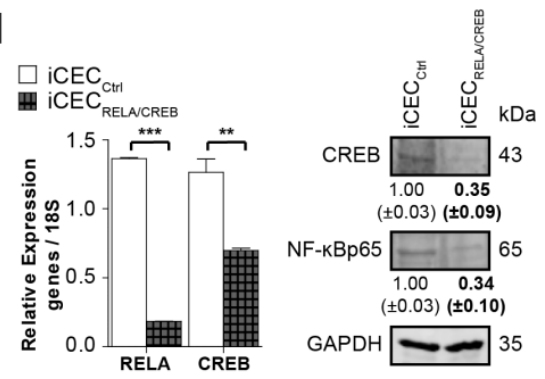

e

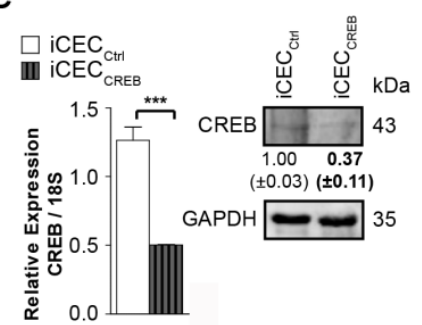

$f$

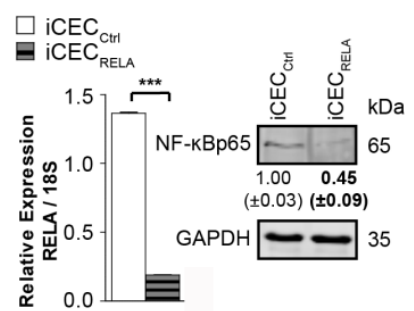

$\mathrm{h}$
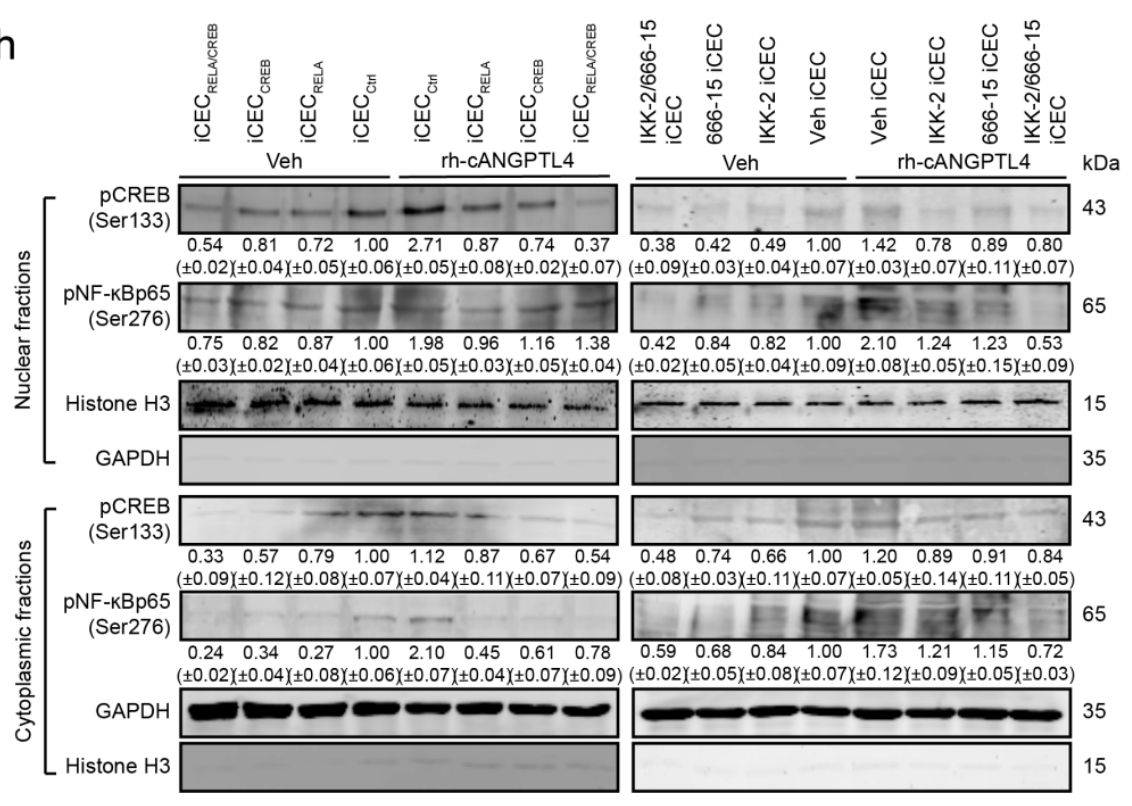

g

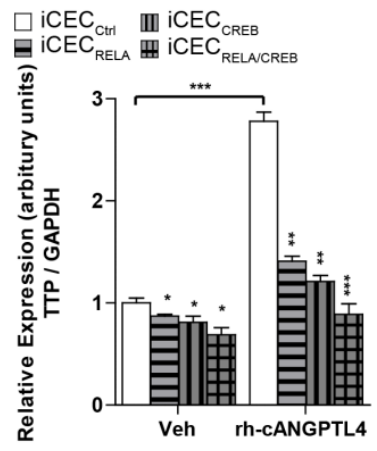

$\square$ Veh iCEC III 666-15 iCEC

日 IKK-2 iCEC 曲 IKK-2/666-15 iCEC

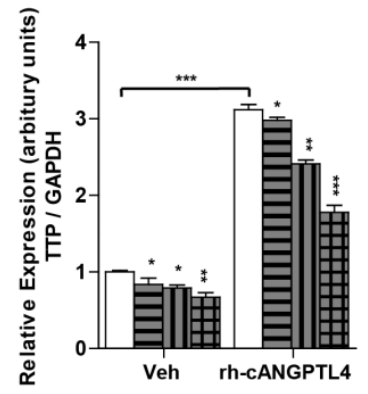




\section{Supplementary Fig. S3. Expression of TTP transcript in iCECs treated with various kinase inhibitors.}

(a) Relative mRNA (left panel) and protein (right panel) levels accounting for TTP expression in iCECs treated with rh-cANGPTL4 and rh-nANGPTL4. (b) A schematic illustration of the rationale behind the kinase inhibitor array, which led up to mapping the IPA pathway in Fig. 4a. When treated with rh-cANGPTL4, TTP expression was increased in iCECs (left panel). Kinase inhibitors $\left(\mathrm{XX}_{\mathrm{n}}\right)$ that negated this up-regulation of TTP in the presence of rh-cANGPTL4 meant that these kinases were directly responsible for regulating TTP expression (middle panel). On the other hand, the addition of kinase inhibitors that brought about an increase in TTP expression in the presence of rh-cANGPTL4 (right panel) suggested that ANGPTL4 works through other unique pathways to up-regulate the expression of TTP, independent of the target kinase. (c) Relative mRNA level of TTP after treatment with various kinase inhibitors from a SYNLibrary 95 array. Relative mRNA (left panel) and protein (right panel) levels accounting for the levels of (d) RELA and CREB, (e) CREB and (f) RELA following siRNA knockdown as determined by real-time PCR and immunoblot analysis, respectively. (g) Relative expression of TTP in iCECs after the depletion of CREB and/or NF- $\kappa \mathrm{B}$ using siRNA knockdown ( $\mathrm{iCEC}_{\mathrm{Ctrl}}, \mathrm{iCEC}_{\mathrm{RELA}}, \mathrm{iCEC}_{\mathrm{CREB}}$ and $\left.\mathrm{iCEC}_{\mathrm{RELA} / \mathrm{CREB}}\right)$ and CREB and/or NF-אB inhibitors (Veh iCEC, IKK-2 iCEC, 666-15 iCEC and IKK-2 /666$15 \mathrm{iCEC}$ ). (h) Immunoblot analysis of pCREB, CREB, and pNF- $\mathrm{B}$ for nuclear (upper panel) and cytoplasmic fractions (lower panel) isolated from iCECs $\left(\mathrm{iCEC}_{\mathrm{Ctrl}}, \mathrm{iCEC}_{\mathrm{RELA}}, \mathrm{iCEC}_{\mathrm{CREB}}\right.$, iCEC $_{\text {RELA/CREB }}$, Veh iCEC, IKK-2 iCEC, 666-15 iCEC and IKK-2 /666-15 iCEC) stimulated with or without rh-cANGPTL4. Mann-Whitney U test was used. Three independent experiments $(\mathrm{n}=3)$ were performed (means \pm s.e.m.); ${ }^{*} \mathrm{p}<0.05$, **p $<0.01,{ }^{* * *} \mathrm{p}<0.001$. 
Microarray Analysis of Murine colon samples

Veh-, DSS- and SA- treated colon samples from ANGPTL $4^{+-}$and ANGPTL4 ${ }^{+/+}$littermates were ran on the Affymetrix GeneChip $\otimes$ Mouse Gene 1.0 ST Array gene array (full genelist deposited under GSE78500). Subsequently,

data sets were imported and analyzed using the

Partek $\circledast^{\circledR}$ Genomics Suite ${ }^{\mathrm{TM}}$ to identify differences in transcriptomic profiles.

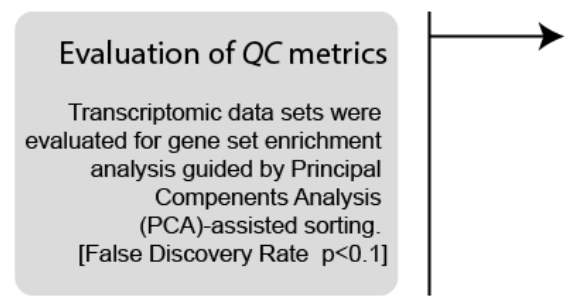

Microarray Analysis of Human Ulcerative Colitis samples

Patient ulcerative colitis (UC) colon samples were initially published by Olsen et al. (GSE9452) were analysed using the Partek ${ }^{\circledR}$ Genomics Suite ${ }^{\mathrm{TM}}$ to identify differences in transcriptomic profiles.

\section{Identification of Genes of Interest}

Transcripts were estimated using a $\log _{2}$ transformation and subjected to a mixed-model analysis of variance (ANOVA) to

detect differentially expressed genes between groups.

Genes with a fold change below -1.2 and above 1.2 were identified ( \pm 1.2 fold-change limit). Subsequently, genes were hierarchical clustered based on significance, and scaled to standard deviation one with genes shifted to mean of zero to produce heat maps.

The gene list representing Veh-, DSS- and SA- treated ANGPTL $4^{-+}$and ANGPTL $4^{+/+}$mice can be located in Fig. 2a-b, Supplementary Table S4, while the merged gene list between mouse and human samples can be located in Fig. 2e-f, Supplementary Table S5.

\section{Gene Ontology (GO) Analysis}

Using the database in Ingenuity Pathways Analysis 5.0 (IPA) platform, we first identified the list of known genes in literature (both direct and indirect, as well as endogenous chemicals) associated with "Gastrointestinal Diseases". Both gene lists (generated from the microarray analysis and and

from IPA) were then merged to identify a list of similarly regulated genes.

- Most significant canonical pathways through which genes belong to were identified. [Fig. $\mathbf{2 c}, \mathbf{g}$ ]

- Most significant molecular, cellular functions and biological processes where by genes are known to participate in were identified. [Fig. 2d, h]

\section{Supplementary Fig. S4. Schematics of the microarray analysis.}

Schematics illustrating the flow of the microarray analysis. Both murine and human samples

were subjected to ANOVA in Partek Genomics Suite software to evaluate and identify

changes in gene transcriptomic profiles. Subsequently, datasets were analyzed using

Ingenuity Pathway Analysis to identify top hits in canonical pathways and biological

processes. 


\section{Loss of TAK1 Increases Cell Traction Force in a ROS-dependent Manner to Drive Epithelial-Mesenchymal Transition of Cancer Cells}

Chee Ren Ivan LAM, Carol TAN, Ziqiang TEO, Chor Yong TAY, Terri PHUA, YunLong WU, Ping Qiang CAI, Lay Poh TAN, Xiao Dong CHEN, Pengcheng ZHU, Nguan Soon TAN*

*Corresponding Author.

Cell Death and Diseases. 2013 Oct 10;4:e848. doi: 10.1038/cddis.2013.339. 


\title{
Loss of TAK1 increases cell traction force in a ROS-dependent manner to drive epithelial- mesenchymal transition of cancer cells
}

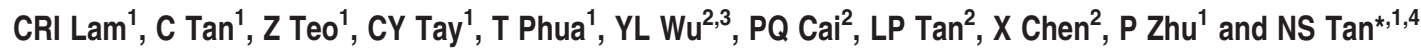

Epithelial-mesenchymal transition (EMT) is a crucial step in tumor progression, and the TGF $\beta$-SMAD signaling pathway as an inductor of EMT in many tumor types is well recognized. However, the role of non-canonical TGF $\beta$-TAK1 signaling in EMT remains unclear. Herein, we show that TAK1 deficiency drives metastatic skin squamous cell carcinoma earlier into EMT that is conditional on the elevated cellular ROS level. The expression of TAK1 is consistently reduced in invasive squamous cell carcinoma biopsies. Tumors derived from TAK1-deficient cells also exhibited pronounced invasive morphology. TAK1-deficient cancer cells adopt a more mesenchymal morphology characterized by higher number of focal adhesions, increase surface expression of integrin $\alpha 5 \beta 1$ and active Rac1. Notably, these mutant cells exert an increased cell traction force, an early cellular response during TGF $\beta 1$-induced EMT. The mRNA level of ZEB1 and SNAIL, transcription factors associated with mesenchymal phenotype is also upregulated in TAK1-deficient cancer cells compared with control cancer cells. We further show that TAK1 modulates Rac1 and RhoA GTPases activities via a redox-dependent downregulation of RhoA by Rac1, which involves the oxidative modification of low-molecular weight protein tyrosine phosphatase. Importantly, the treatment of TAK1-deficient cancer cells with Y27632, a selective inhibitor of Rho-associated protein kinase and antioxidant $N$-acetylcysteine augment and hinders EMT, respectively. Our findings suggest that a dysregulated balance in the activation of TGF $\beta$-TAK1 and TGF $\beta$-SMAD pathways is pivotal for TGF $\beta 1$-induced EMT. Thus, TAK1 deficiency in metastatic cancer cells increases integrin:Rac-induced ROS, which negatively regulated Rho by LMW-PTP to accelerate EMT.

Cell Death and Disease (2013) 4, e848; doi:10.1038/cddis.2013.339; published online 10 October 2013

Subject Category: Cancer

Metastasis accounts for more than $90 \%$ of cancer-related deaths. ${ }^{1}$ Metastasis is a multi-stage process involving cancer cell motility, intravasation, transit in the blood or lymph, extravasation and growth at a new site. ${ }^{1}$ Conceivably, the various stages consist of regulatory networks with many opportunities for cancer therapy. The potential for new therapeutics avenues and their clinical impact to reduce cancer mortality rate underscores the immense importance to identify novel target modulators of these regulatory networks during metastasis.

Numerous evidences showed metastasis of epithelial cancer involve the conversion of primary cancerous epithelial cells to a migratory form, that is, undergoes epithelialmesenchymal transition (EMT) for their dissemination to secondary tumor sites. ${ }^{2}$ It is a process whereby cobblestone-shaped epithelial cell colonies acquire distinct mesenchymal traits such as the loss of E-cadherin and the upregulation of $\mathrm{N}$-cadherin to weaken cell-cell junctional adhesion, augmenting cell dissociation. ${ }^{2}$ EMT also exhibit extensive cytoskeletal remodeling, whereby actin stress fibers are formed in conjunction with an increased establishment of integrin-containing focal contacts, effectively enhancing cell attachment to the extracellular matrix. Cancer cells undergoing EMT are also enriched with mesenchymal type intermediate filaments (i.e. desmin, vimentin). The expression of mesenchymal features and suppression of epithelial traits are induced by early EMT-associated transcriptional factors such as SNAIL and ZEB1. Studies suggested that this mesenchymal transformation enhances the ability of cancer cells to intravasate and disseminate. Thus, the identification of mechanistic drivers of EMT would provide crucial insights and therapeutic targets to halt cancer spreading.

There are many evidences that clearly support a tumorpromoting role of inflammation in the development and progression of epithelial cancer. ${ }^{3}$ Data from various animal models clearly emphasize that the tumor-promoting microenvironment is indispensable for tumor formation and progression. Although EMT culminates from an integrated

\footnotetext{
${ }^{1}$ School of Biological Sciences, Nanyang Technological University, 60 Nanyang Drive, Singapore; ${ }^{2}$ School of Materials Science and Engineering, Nanyang Technological University, 50 Nanyang Avenue, Singapore; ${ }^{3}$ School of Pharmaceutical Science, Xiamen University, Xiamen, China and ${ }^{4}$ Institute of Molecular and Cell Biology, 61 Biopolis Drive, Proteos, Singapore

${ }^{*}$ Corresponding author: NS Tan, School of Biological Sciences, Nanyang Technological University, 60 Nanyang Drive, Singapore 637551, Singapore. Tel: +65 6316 2941; Fax: +65 6791 3856; E-mails: nstan@ntu.edu.sg or nstan@imcb.a-star.edu.sg

Keywords: reactive oxygen species; cell traction force; epithelial-mesenchymal transition

Abbreviations: A5RT3 $\mathrm{CTRL}$, control- knockdown A5RT3 cancer cells; A5RT3 ${ }_{T A K 1}$, TAK1-knockdown A5RT3 cancer cells; CAFs, cancer-associated fibroblasts; CTF, cell traction force; EMT, epithelial-mesenchymal transition; NF $\kappa$ B, nuclear factor kappa B; PLA, Proximity ligation assay; PNS, perilesional normal skin; qPCR, quantitative PCR; ROS, reactive oxygen species; SCC, squamous cell carcinomas; TGF $\beta$, transforming growth factor- $\beta$; TAK1, TGF $\beta$ activated kinase 1 Received 19.2.13; revised 23.8.13; accepted 25.8.13; Edited by A Finazzi-Agró
} 
cascade of proinflammatory cytokines, much more remains to be understood about the intracellular signaling pathways that were essential for EMT. Of the numerous cytokines produced at the tumor microenvironment, transforming growth factor- $\beta$ (TGF $\beta$ ) has the broadest effects, influencing many aspects of tumorigenesis. ${ }^{4}$ TGF $\beta$ has a leading role in eliciting inflammation, serving as a chemotactic factor to recruit immune cells. TGF $\beta$ triggers EMT in tumor cells through the canonical Smad signaling.,6 TGF $\beta$ also signals through the non-canonical TGF $\beta$ activated kinase 1 (TAK1) cascade, which is a pivotal proinflammatory signaling mediator. However, the role of TGF $\beta$-mediated TAK1 signaling in EMT is unclear. A hallmark of inflammation is an increase in reactive oxygen species (ROS), which also has a protumorigenic role. ${ }^{7,8}$ TAK1 has been shown to modulate ROS level in normal and tumor cells. The ablation of TAK1 causes accumulation of ROS, resulting in epithelial cell death and inflammation. ${ }^{9,10}$ However, the effect of ROS on EMT is not well understood. In this study, we investigated the role of TAK1 in TGF $\beta$-induced EMT of tumor cells. We found that TAK1 deficiency in tumor cells accelerated EMT in ROS-dependent manner, thus providing a previously unexplored mechanistic regulation of EMT by TAK1.

\section{Results}

Reduced expression of TAK1 in invasive human squamous cell carcinomas (SCC). To assess the clinical relevance of TAK1, we examined the expression of TAK1 from 15 clinically graded SCCs. Tumors were formerly graded by in-house pathologist for their invasiveness, and were grouped into benign or invasive group. We observed reduced $\mathrm{mRNA}$ and protein levels of TAK1 in invasive SCCs when compared with its perilesional normal skin (PNS), which served as cognate-paired control. No difference was observed between benign SCCs and its corresponding PNS (Figures $1 \mathrm{a}$ and $\mathrm{b}$ ). This observation suggests reduced TAK1 expression is associated with an invasive prognosis.

TAK1-deficient A5RT3 cells form skin tumors with invasive morphology. Next we set out to investigate the in vivo effect of TAK1 deficiency on tumor invasiveness by using SSC orthotopic nude mice model. In the first instance, we suppressed TAK1 expression in human SCC A5RT3 cell line using lentiviral-mediated RNAi (designated herein A5RT3 ${ }_{\text {TAK } 1}$ ). A5RT3 cells transduced with a scrambled sequence served as the control A5RT3 ${ }_{\text {CTRL. The efficiency }}$ of TAK1-knockdown was verified with immunoblotting and quantitative PCR (qPCR) (Supplementary Figure S1A). Immunoblot analysis of A5RT3 that diminished TAK1 corresponded with a reduced downstream phosphorylation of nuclear factor kappa $\mathrm{B}(\mathrm{NF} \kappa \mathrm{B})$ upon treatment with interleukin-1 (IL-1) ${ }^{4}$ (Supplementary Figure S1B). The activation of other stress response pathways like ERK and JNK were likewise also found to be suppressed in A5RT3 ${ }_{\text {TAK1 }}$ (Supplementary Figure S1C). $\mathrm{A} \mathrm{RT} 3_{\mathrm{CTRL}}$ and $\mathrm{A} \mathrm{RT} 3_{\mathrm{TAK} 1}$ cells showed no significant difference in their rate of proliferation (Supplementary Figure S1D). A5RT3 $3_{\text {TAK1 }}$ and A5RT3 ${ }_{\text {CTRL }}$ were injected subcutaneously into nude mice to induce tumor formation.
A5RT3 $3_{\text {TAK } 1-\text { and }}$ A5RT3 $_{\text {CTRL-derived tumors were harvested }}$ and sections were immunostained for laminin-332, a recognized marker of tumor invasiveness. ${ }^{11-13}$ Laminin-332 immunostaining in A5RT3 $_{\text {CTRL-derived tumors appeared as }}$ demarcated boundaries characteristic of basement membrane type architecture, whereas the corresponding staining

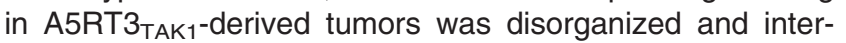
spersed among the tumor epithelial cells, indicative of enhanced invasiveness. This spatial disparity in laminin332 immunostaining also corresponded with an increased laminin-332 protein (Figure 1d). Gelatin zymography also revealed increased MMP-9 activity, another marker of tumor invasiveness, in A5RT3 ${ }_{\mathrm{TAK} 1}$-tumor. These observations indicate that the TAK deficiency is associated with enhanced invasiveness (Figure 1d).

TAK1 deficiency promotes TGF $\beta-1$ induced EMT in A5RT3 cells. Reduced TAK1 expression is a common attribute of invasive human SCCs. In addition, TAK1deficient tumors exhibited more prominent invasive features. The TGF $\beta 1-S M A D$ signaling pathway is central to EMT induction. However, the role of TGF $\beta 1-T A K 1$ signaling in EMT remains unknown. A5RT3 ${ }_{T A K 1}$ colonies treated with TGF $\beta 1$ showed earlier separation at cell-cell borders at $48 \mathrm{~h}$ post treatment (Figure 1e). Immunofluorescence staining revealed a decreased E-cadherin and a corresponding increased $\mathrm{N}$-cadherin staining (Figure 1f). The mRNA levels of EMT markers measured by qPCR revealed an EMT phenotype as early as $24 \mathrm{~h}$ after exposure in A5RT3 treated with TGF $\beta$ compared with cognate controls (Figure 1g). The protein expression of EMT markers vimentin, fibronectin, Snail 1 and Snail 2 was also altered, indicating an earlier switch to an EMT phenotype by A5RT3 ${ }_{\text {TAK1 } 1}$ cells (Figure 1h). To further assess the potential role of TAK1 in EMT, we examined TGF $\beta 1$-induced EMT in three other tumor lines, HSC-5, II4 and MKN28, whose endogenous TAK1 was suppressed by SiRNA. HSC-5 was established from a clinically derived human squamous cell carcinoma. ${ }^{14}$ II4 cell line is a Ras-transformed HaCat cell line that is not metastatic. ${ }^{15}$ MKN28 is a well-differentiated stomach adenocarcinoma cell line. ${ }^{16}$ Importantly, we also observed similar augmentation of TGF $\beta 1$-induced EMT in these TAK1-knockdown cells. TAK1-deficient cells were observed to adopt mesenchymal-like cell-cell separation upon TGF $\beta 1$ induction (Supplementary Figure S1E) and corresponded to the expression of mesenchymal markers (Supplementary Figure S1F). These observations support a role of TAK1 deficiency in promoting mesenchymal transition. We also found that TGF $\beta$-induced EMT phenotype was prevented in $\mathrm{A} 5 \mathrm{RT} 3_{\mathrm{TAK} 1}$ transfected with an expression vector for TAK1 pCDNA-TAK1 (Supplementary Figure S2). The TAK1 mRNA sequence transcribed from pCDNA-TAK1 contains silent mutations that are not targeted by the siRNA produced in A5RT3 TAK1 $_{\text {. }}$.

To understand the relationship between SMAD and TAK1 in TGF $\beta 1$-induced EMT, we assessed the relevance of SMAD3 in TGF $\beta 1$-induced EMT of A5RT3 ${ }_{\text {TAK } 1}$ cells, which was previously unclear. A5RT3 ${ }_{\mathrm{TAK} 1}$ cells were transiently transfected with siRNA targeting SMAD3 (Figure 2). Loss of

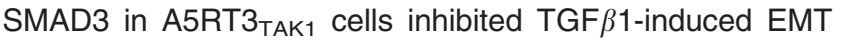




\section{a}
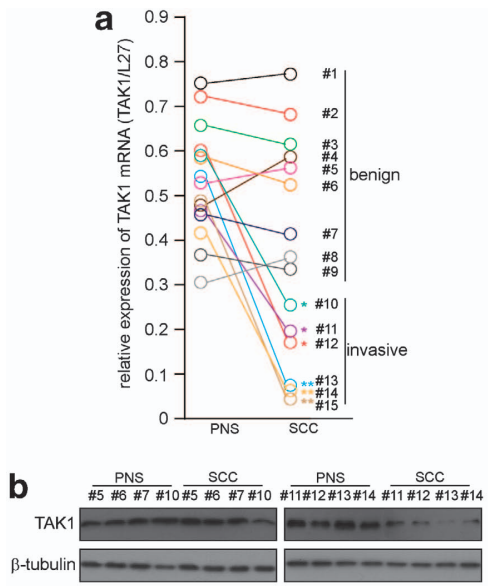

e
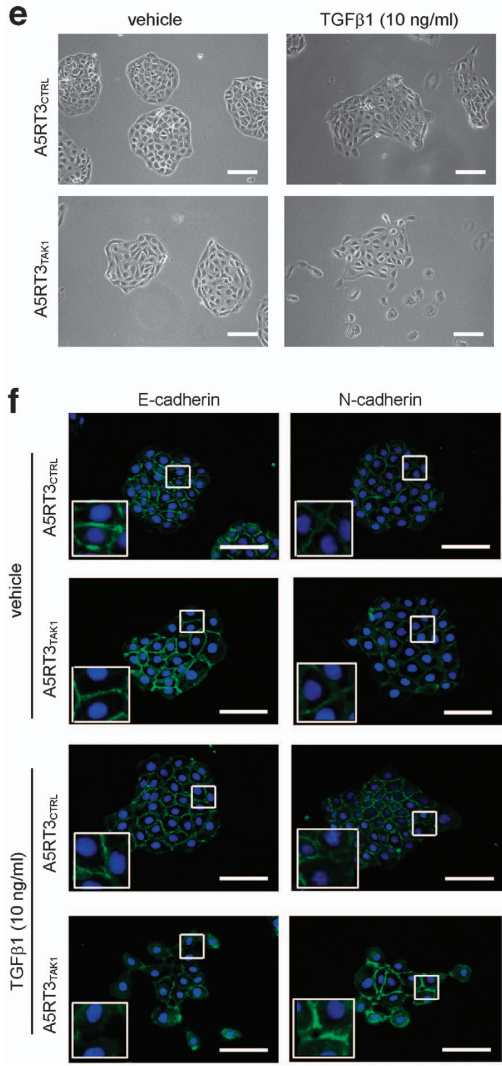

C $\quad$ ASRT $_{\text {CTRL }}$

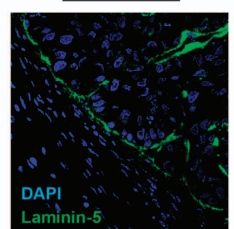

A5RT3 $_{\text {TAK } 1}$

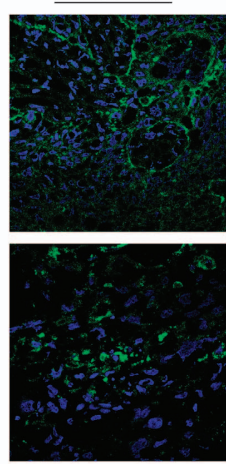

d
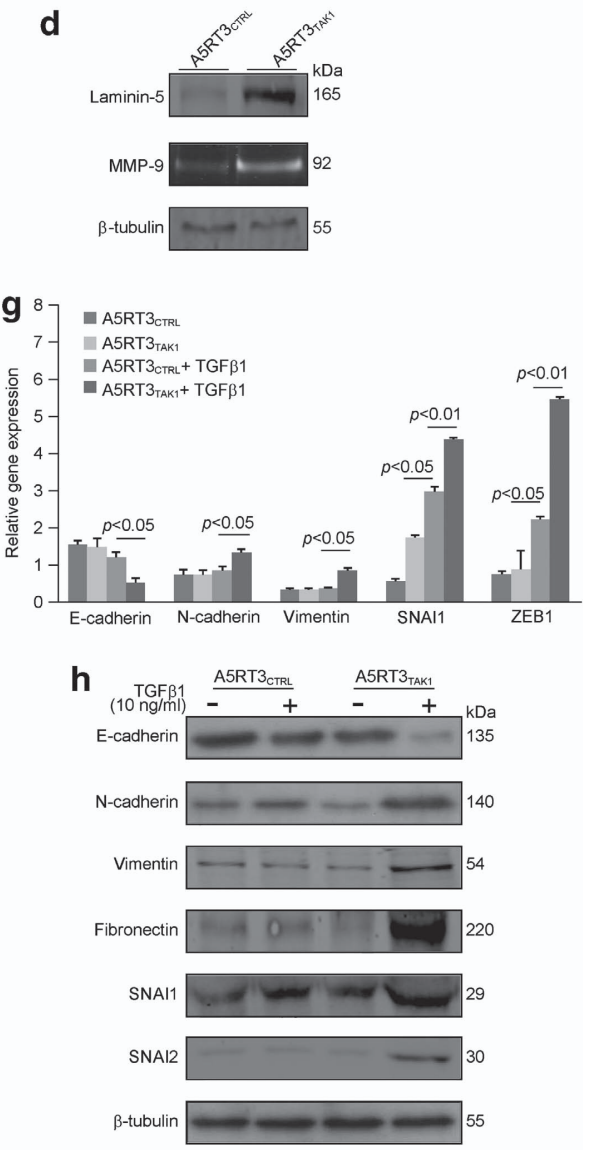

Figure 1 TAK1 deficiency promotes TGF $\beta 1$ induced EMT in A5RT3 cells. ( $\mathbf{a}$ and $\mathbf{b}$ ) Relative mRNA (a) and protein (b) expression of TAK1 in human squamous cell carcinomas (SCC) biopsies and their cognate perilesional normal skin (PNS). Biopsies \#1-9 and \#10-15 are benign and invasive grades SCC samples, respectively. Biopsies \#5-7 and \#10-14 were used for western blot analysis. For qPCR, data points from the same individual are linked by colored lines. (c) Immunofluorescence staining of laminin332 (green) in cryosections of A5RT3 CTRL- and A5RT3 ${ }_{\text {TAK1 }}$-derived tumors. Nuclei were counterstained with DAPI (blue). (d) Expression of invasive markers laminin-332 and MMP-9 in A5RT3 CTRL $^{-}$and A5RT3 ${ }_{T A K}$-derived tumors as determined by immunoblot and gelatin zymography, respectively. Values below each band represent the mean fold differences $(n=3)$ in expression level when compared with A5RT3 ${ }_{\text {CTRL }}$-derived tumor. (e) Phase-contrast microscopy images of A5RT3 ${ }_{\text {CTRL }}$ and A5RT3 ${ }_{\text {TAK1 } 1}$ cell colonies $48 \mathrm{~h}$ after TGF $\beta 1$ treatment. Scale bar, $100 \mu \mathrm{m}$. (f) Immunostaining of E-cadherin and N-cadherin (green) in A5RT3 ${ }_{\text {CTRL }}$ and A5RT3 ${ }_{\text {TAK } 1}$ cells under the indicated treatment. Nuclei were counterstained with DAPI (blue). Scale bar, $100 \mu \mathrm{m}$. (g) qPCR analysis of EMT markers in A5RT3 ${ }_{\text {CTRL }}$ and A5RT3 ${ }_{\text {TAK1 } 1} 24 \mathrm{~h}$ after TGF $\beta 1$ treatment. Samples were normalized with reference gene, L27. (h) Representative western blots of EMT markers in A5RT3 ${ }_{\text {CTRL }}$ and A5RT3 ${ }_{\text {TAK } 1} 48 \mathrm{~h}$ after TGF $\beta 1$ treatment. The densitometry values as indicated below respective lanes were normalized with respect to the control. For western blot, $\beta$-tubulin was used as a loading control. For qPCR, all samples were normalized with housekeeping gene L27 gene. Data represent means \pm S.D.; $n=3$

(Figure 2a). The expression of mesenchymal markers was downregulated whereas epithelial markers were upregulated in SMAD3-deficient A5RT3TAK1 cells (Figures 2b and c). We observed that EMT also occurred in A5RT3 after prolonged (i.e. $72 \mathrm{~h}$ ) treatment with TGF $\beta 1$ as compared

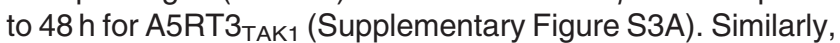
SMAD3 deficiency in A5RT3 ${ }_{\text {CTRL }}$ cells inhibited TGF $\beta 1$ induced EMT (Supplementary Figures S3B \& S3C). 
a
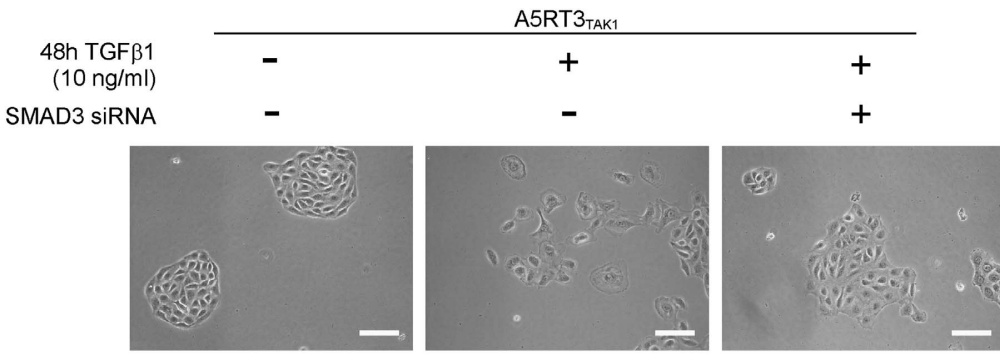

b

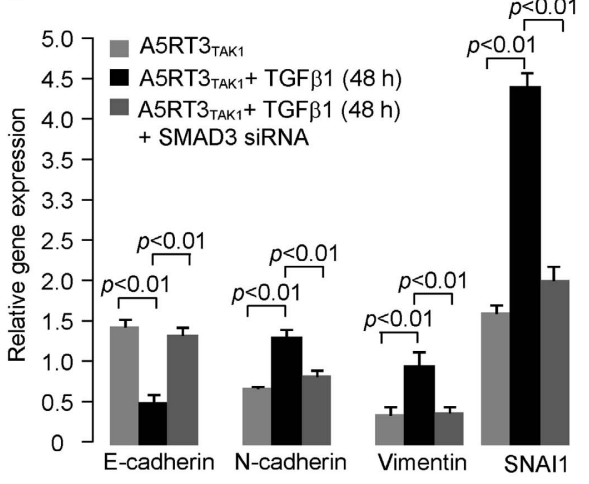

c

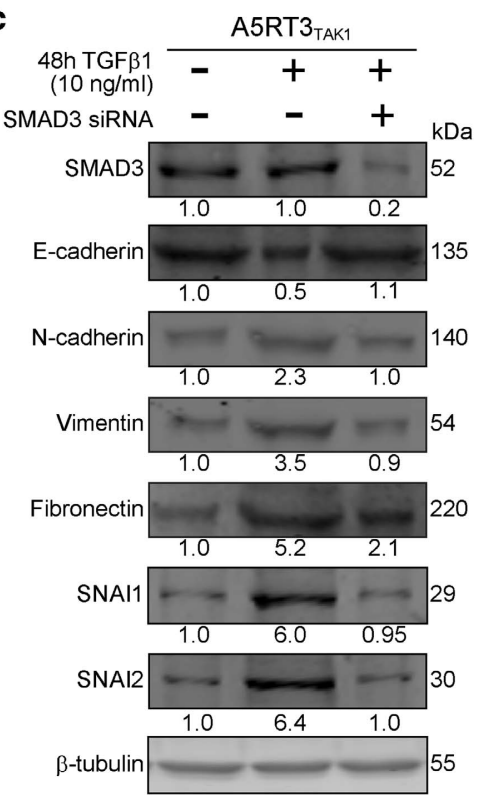

Figure 2 TGF $\beta 1$-induced EMT is effected through SMAD signaling. (a) Phase-contrast images of SMAD3 siRNA-transfected A5RT3 ${ }_{\text {TAK } 1}$ and respective control cells with $48 \mathrm{~h}$ of TGF $\beta 1(10 \mathrm{ng} / \mathrm{ml})$ treatment. Scale bar, $100 \mu \mathrm{m}$. (b) qPCR analysis of EMT markers in SMAD3 siRNA-transfected A5RT3 TAK1 cells with and without TGF $\beta 1$ treatment. (c) Immunoblot analysis of SMAD3 siRNA-transfected A5RT3 ${ }_{\text {TAK } 1}$ cells for their knockdown and EMT markers upon TGF $\beta 1$ treatment. Representative blots were shown with respective densitometry values indicated below lanes, normalized with respect to the control. For western blot, $\beta$-tubulin was used as a loading control. For qPCR, all samples were normalized with housekeeping gene L27 gene. Data represent means \pm S.D.; $n=3$

The phosphorylation of SMAD3 was also evaluated in the SMAD3-knockdown cells by immunoblotting (Supplementary Figure S3D). Altogether, our observations indicates that TAK1 limits TGF $\beta$ driven pro-EMT signals mediated by SMAD3 pathway, and suggests a signaling balance between the canonical SMAD3 and non-canonical TAK1 in regulating EMT.

TAK1 deficiency increases the expression of surface integrin $\beta 1$ and $\beta 3$. Our above observations suggest that TAK1 deficiency in A5RT3 facilitated TGF $\beta 1$-induced EMT. EMT involves the transition of the epithelial cancer cells from predominantly cell-cell adhesion to a cell-ECM interaction. Integrins are major cell surface receptors that enable bidirectional communication between the cells with the ECM are thus well apt to influence cellular behavior involved in EMT including migration and adhesion. ${ }^{17,18}$ Thus, we examined the surface expression of integrins $\beta 1, \beta 3, \beta 4, \beta 5$ and $\beta 7$ on A5RT $_{\text {TAK } 1}$ (Figure 3a). We found that A5RT3 $3_{\text {TAK1 }}$ had significantly increased surface levels of $\beta 1$ and $\beta 3$ integrins (Figure 3a). We further conducted staining of $\alpha 5 \beta 1$ integrin and found that it is also significantly elevated in a manner reminiscent of integrin $\beta 1$ (Figure $3 b$ ). Proximity ligation assay (PLA) also confirmed higher level of surface $\alpha 5 \beta 1$ integrin in A5RT3 $_{\text {TAK } 1}$ than A5RT3 ${ }_{\text {CTRL }}$ (Figure 3c, upper panel). PLA assay was conducted without Triton-X permeabilization to detect only to surface integrin levels. PLA signals for integrin $\alpha 5 \beta 1$ were predominantly situated along the cell-cell boundary (Figure 3c, upper panel). PLA assay performed using antibody against active integrin $\beta 1$ and configuration-specific monoclonal anti-Rac1-GTP antibody further confirmed that the increase expression of surface integrin was associated with an elevated Rac1 activation, suggesting a remodeling of the cytoskeleton (Figure 3c, lower panel).

Loss of TAK1 increases cell traction force in A5RT3 during EMT. During EMT, integrins are recruited to distinct contact points to form focal adhesions accompanied by cytoskeletal remodeling of cortical actin network to filamentous stress fibers. Interestingly, we already observed more focal adhesions and better developed stress fiber formation in A5RT3 ${ }_{\text {TAK1 }}$ than A5RT3 $3_{\text {CTRL, }}$ before TGF $\beta 1$ treatment (Figure 4a). These differences in stress fiber formation correlated with vinculin-positive focal adhesion staining at their cell junctions of A5RT3 TAK1 $_{1}$ (Figure 4a). The vinculin 

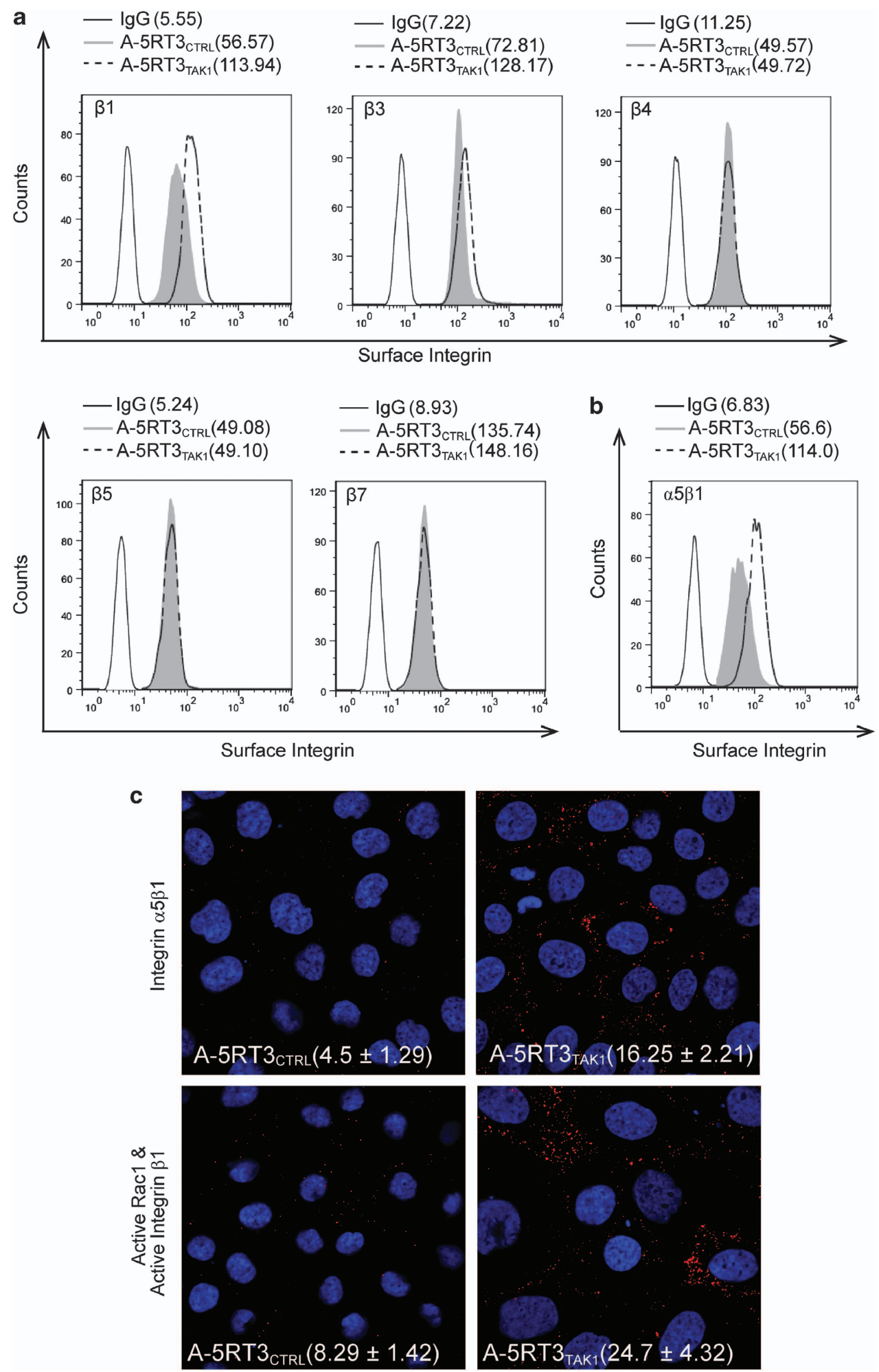

Figure 3 Expression of surface integrin in A5RT3 ${ }_{\text {CTRL }}$ and A5RT3 ${ }_{\text {TAK1. }}$ ( $\mathbf{a}$ and $\left.\mathbf{b}\right)$ FACS analysis of A5RT3 $3_{C T R L}$ and A5RT3 $3_{\text {TAK1 }}$ cells immunostained with antibodies against indicated surface integrin $\beta$ subunits (a) and with integrin $\alpha 5 \beta 1$ (b). The distribution of tumor cells with regard to their extent of integrin staining was presented in histogram plots with the identity of the stained integrin subunit indicated (top left). Cells stained with only control IgG served as the negative controls. Each image is representative of at least three different experiments. Values shown indicate mean fluorescence intensity. (c) PLA assay of $\alpha 5 \beta 1$ integrin expression (upper panel) and active integrin $\beta 1$ :active Rac1 (lower panel) n A5RT3 ${ }_{\mathrm{CTRL}}$ and A5RT3 ${ }_{\mathrm{TAK} 1}$ cells. Representative images were shown with mean number of PLA spots per nucleus \pm S.D. indicated; $n=3$ 

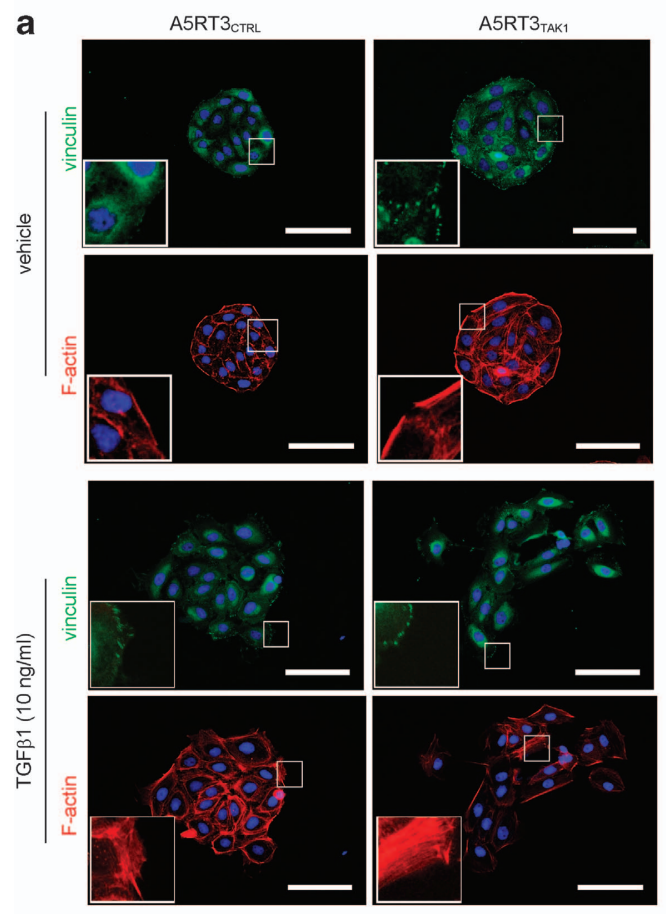

b
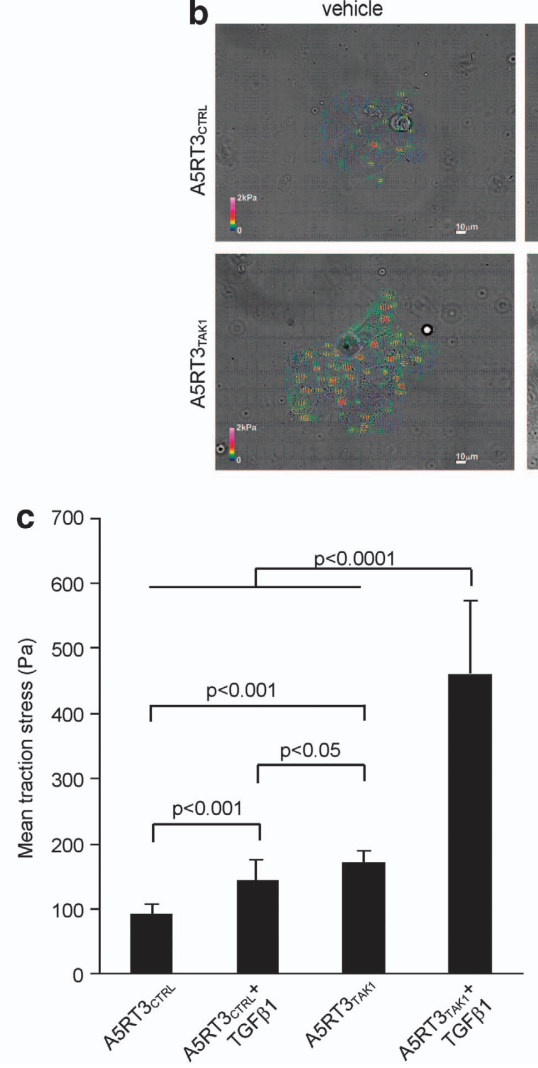

$\operatorname{TGF} \beta 1(10 \mathrm{ng} / \mathrm{ml})$
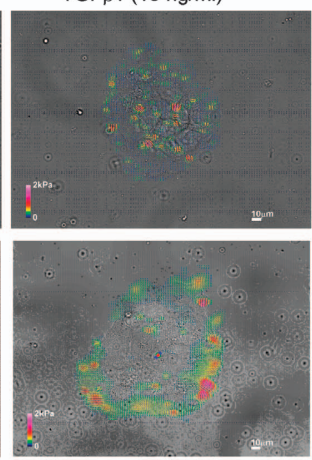

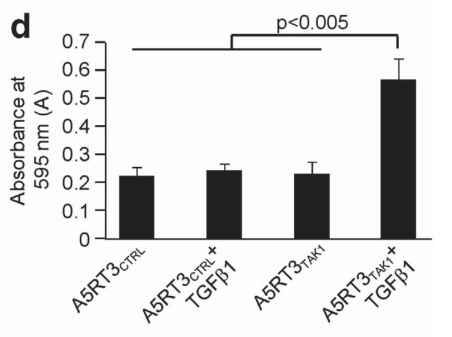

Figure 4 TAK1 deficiency increases cell traction force in A-5RT3 facilitating EMT. (a) Vinculin immunostaining (green) and phalloidin F-actin (red) staining were conducted of A5RT3 $3_{\text {CTRL }}$ and A5RT3TAK1 cell colonies with and without TGF $\beta 1$ treatment for $48 \mathrm{~h}$ with representative images shown. Scale bar, $100 \mu \mathrm{m}$. (b and $\left.\mathbf{c}\right)$ Cell traction force profiling (b) and mean measured cell traction stress (c) of A5RT3 ${ }_{\text {CTRL }}$ and A5RT3 TAK1 with and without TGF $\beta 1$ ( $\left.10 \mathrm{ng} / \mathrm{ml}\right)$ treatment for $24 \mathrm{~h}$. Color scale bar denotes traction stress $(\mathrm{kPa})$. Scale bar, $10 \mu \mathrm{m}$. Values (mean + S.D.) of three independent measurements. (d) A5RT3 ${ }_{\mathrm{CTRL}}$ and A5RT3 ${ }_{\text {TAK1 }}$ cells were seeded on a transwell membrane of pore size and evaluated for their migration through the pore with and without TGF $\beta 1$ treatment for $48 \mathrm{~h}$. Migrated cells were stained with crystal violet and destained with a fixed volume of $0.5 \%$ Triton-X solution. Absorbance at $595 \mathrm{~nm}$ of the solutions was measured to quantify transwell migration. Graph displays mean absorbance values \pm S.D.; $n=3$

staining also displayed differences in spatial distribution observed in PLA staining for integrin $\alpha 5 \beta 1$. As expected, the treatment with TGF $\beta 1$ increased stress fiber in both cell lines despite their different cell-cell dispersion. Differences in stress fibers and focal adhesion contact formation in cells are known to effect differential cell traction force (CTF) generation. These force differences represent the early detectable changes in cell responses, preceding the appearance of visible morphological changes. Thus, we measured the CTF generated in A5RT3 TAK1 $_{\text {and }}$ A5RT3 ${ }_{\text {CTRL using CTF micro- }}$ scopy. Cells were cultured on fibronectin-treated compliant PDMS substrate $(10 \mathrm{kPa})$ coated with a monolayer of fluorescence beads. Traction force is determined by measuring the displacement of the fluorescence beads in the $x-y$ plane because of the contraction exerted by the cell. Notably, A5RT3 ${ }_{\text {TAK1 } 1}$ generated a considerably different CTF profile from that of control A5RT3 $3_{C T R L}$ cells. Although traction field was observed to be concentrated along the periphery of the A5RT3 ${ }_{\text {CTRL }}$ cell colony, intra-colony traction can only be detected in the A5RT3 ${ }_{\text {TAK1 }}$ group (Figure 4b). As the CTF exerted is also relative to the cell colony size and shape, measured CTF values are divided over the area covered by the cell colonies to derive traction stress values for comparison between cell colonies of differing sizes.
Untreated A5RT3 ${ }_{\text {CTRL }}$ cells displayed a mean traction stress value of $93.4 \pm 14.8 \mathrm{~Pa}$, whereas that of A5RT3 ${ }_{\text {TAK1 }}$ displayed a mean CTF of $170.7 \pm 20.2 \mathrm{~Pa}$ (Figure 4c), indicating higher contractility in TAK1-deficient cancer cells. TGF $\beta 1$ induction resulted in significant CTF increments for both $\mathrm{A}_{5 \mathrm{RT}} 3_{\mathrm{CTRL}}$ and $\mathrm{A}_{5 \mathrm{RT}} \mathrm{T}_{\mathrm{TAK} 1}$ as reflected in their elevated traction stress values of $143.96 \pm 31.7 \mathrm{~Pa}$ and $461.4 \pm 113.22 \mathrm{~Pa}$, respectively (Figure 4c). Notably,

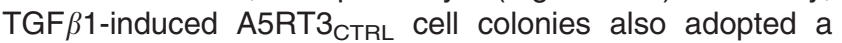
CTF profile pattern comparable with that of untreated A5RT3 ${ }_{\text {TAK1. In }}$ transwell invasion assay, we found that A5RT3 TAK1 significantly migrated faster through an $8-\mu \mathrm{m}$ pore transwell with TGF $\beta 1$ as the chemotactic factor (Figure $4 d$ ). Our results indicate that TAK1 deficiency in A5RT3 promoted the expression of $\beta 1$ and $\beta 3$ integrins, and altered stress fiber formation. This cytoskeletal remodeling corresponded with an increased CTF profile and metastatic potential in A5RT3 ${ }_{\text {TAK1 }}$ cells, suggesting an inhibitory role of endogenous TAK1 during TGF $\beta 1$-induced EMT.

Elevated ROS levels in TAK1-deficient tumor cells accelerate EMT. TAK1 deficiency was reported to upregulate ROS level in human keratinocytes and in cancer cells. ${ }^{9,19}$ Integrin-Rac1 signaling mediates multiple pathways 
that control actin cytoskeletal changes, transcriptional activity and ROS production. ${ }^{20,21}$ First, we examine if the increased

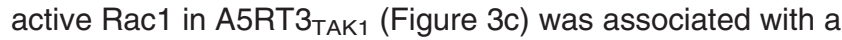
higher recruitment of Rac1-dependent Nox1 for ROS generation. PLA assay using antibodies against active Rac1 and Nox1 revealed higher number of PLA signals in

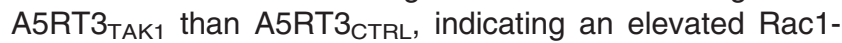
dependent activation of Nox1 (Figure 5a). Next, we evaluated intracellular ROS production in A5RT3 ${ }_{T A K}$ and A5RT3 ${ }_{\text {CTRL }}$ using fluorescence dye CM-H2DCFDA followed by FACS analysis. We found that A5RT3 TAK1 cells have increased ROS level, which was further potentiated by TGF $\beta 1$ induction. In fact, the ROS level in untreated

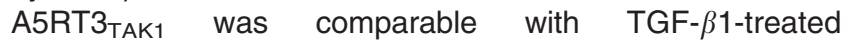
A5RT3 $_{\text {CTRL }}$ (Figure $5 \mathrm{~b}$ ). The difference in ROS level between A5RT3 $_{\text {CTRL }}$ and A5RT3 TAK $1_{\text {upon TGF }} \beta 1$ treatment was also verified by fluorescence microscopy. Interestingly, we observed that A5RT3 ${ }_{\text {TAK } 1}$ cells that have separated from the tumor colony were more intensely stained with $\mathrm{CM}$ -

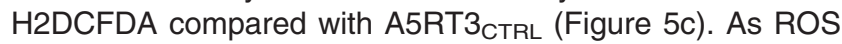
upregulation in $A 5 R T 3_{T A K 1}$ correlated with the mesenchymal phenotype induced by TGF $\beta 1$, it was conceivable that the observed elevated oxidative stress may be mechanistically involved in EMT. Antioxidant $\mathrm{N}$-acetylcysteine (NAC) addition was utilized to quench ROS. First, we determine the optimal concentration of NAC $(30 \mu \mathrm{M}-30 \mathrm{mM})$ for our experiments. At NAC concentrations of $3-30 \mathrm{mM}$, annexin $\mathrm{V} /$ propidium iodide staining of $\mathrm{A}_{5 \mathrm{R}} \mathrm{3}_{\mathrm{TAK} 1}$ revealed increased percentage of apoptotic cells (Supplementary Figure S4A). A corresponding CM-H2DCFDA staining showed that the ROS level was increased in cells treated with high $30 \mathrm{mM}$ NAC concentration, most likely due to cell death. The treatment with $300 \mu \mathrm{M}-3 \mathrm{mM}$ of NAC did not lower the ROS further than that obtained by $100 \mu \mathrm{M}$ of NAC. At $30 \mu \mathrm{M}$ of NAC, the decrease in ROS was less effective (Figure 4b). Thus, we determine the optimal NAC concentration to be $100 \mu \mathrm{M}$, which lowers the ROS level without substantially increasing cell death. We quenched the ROS in the cancer cells with NAC and observed that the treatment hindered the cell-cell separation characteristic of TGF $\beta 1$-induced EMT in A5RT3 ${ }_{\text {TAK1 }}$ (Figure $5 \mathrm{~d}$ ). The NAC-treated cells were significantly diminished of their EMT traits (Figures $5 e$ and f). NAC treatment neither altered the phenotype of A5RT3 ${ }_{C T R L}$ cells (Supplementary Figure S4C) nor elicited any off-target effects as verified by immunoblotting of various EMT markers (Supplementary Figure S4D).

Inhibition of Rho accelerated TGF $\beta 1$-induced EMT in A5RT3 $_{\text {TAK1. }}$. Our above observations on increased CTF of untreated A5RT3 $3_{\text {TAK1 } 1}$ corresponded with the increased surface integrin expression, focal adhesion and stress fiber formation. RhoA and Rac1 have key roles in the formation of stress fiber, focal adhesion, cytoskeleton organization and cellular migration. We first examined their activities in A5RT3 $_{\text {CTRL }}$ and A5RT3 $_{\text {TAK1 } 1}$ with and without TGF $\beta 1$ induction. In both TGF $\beta 1$-treated and -untreated groups, the activities of Rac1 and RhoA were elevated and reduced, respectively, in $A 5 R T 3_{\text {TAK1 }}$ compared with $A 5 R T 3_{C T R L}$ (Figure 6a). We observed that NAC treatment increased RhoA activity, but has not significantly affected the level of active Rac1 (Figure 6a). This is consistent with our earlier observation that increased surface expression of integrin $\beta 1$ activated Rac1, which subsequently engaged Nox1 for ROS generation. It also suggested that the activity of RhoA was redox-dependent. To further understand the relationship between Rac1 and ROS, we transfected A5RT3 ${ }_{C T R L}$ and A5RT3 $3_{\text {TAK } 1}$ with expression vectors containing constitutively active (G12V) and dominant-negative Rac mutant $(\mathrm{T} 17 \mathrm{~N})$, respectively. FACS analysis revealed that A5RT3 ${ }_{\text {CTRL }}(\mathrm{G} 12 \mathrm{~V})$ cells have increased ROS content compared with control vector transfected A5RT3 ${ }_{C T R L}$ (Figure 6b). Conversely, the oxidative stress level of A5RT3 ${ }_{\text {TAK1 }}(\mathrm{T} 17 \mathrm{~N})$ was significantly decreased compared

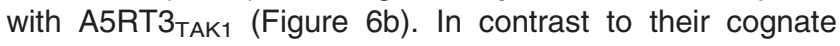
parent cell lines, A5RT3 ${ }_{\mathrm{CTRL}}(\mathrm{G} 12 \mathrm{~V})$ and $\mathrm{A}_{5 \mathrm{RT}} \mathrm{T}_{\mathrm{TAK} 1}(\mathrm{~T} 17 \mathrm{~N})$ showed increased and reduced TGF $\beta 1$-induced EMT, respectively (Figure 6c).

To understand how ROS can modulate the activity of RhoA, we employed the sensitive cysteine-targeted biotin switch method to label oxidatively modified proteins. We showed that the oxidation of low-molecular weight protein tyrosine phosphatase (LMW-PTP), a potential downstream target of ROS was significantly increased in A5RT3 ${ }_{\text {TAK1. }}$. LMW-PTP oxidation was, however, undetectable in NAC-treated A5RT3 ${ }_{\text {TAK } 1}$ cells (Figure $6 \mathrm{a}$ ) regardless of TGF $\beta 1$ treatment. Oxidatively modified LMW-PTP has been shown to inhibit RhoA activity. ${ }^{20}$ To understand the role of RhoA in TGF $\beta 1$ induced EMT, we treated A5RT3 TAK1 $_{\text {with }}$ Y27632, a selective inhibitor of Rho-associated protein kinase, the downstream mediator of RhoA. The inhibition of Rho augmented EMT in A5RT3 ${ }_{\text {TAK1 } 1}$ cells detected within $24 \mathrm{~h}$ post-TGF $\beta 1$ treatment (Figure 6d). The Y27632-treated cells had decreased epithelial marker expression and increased expression of mesenchymal markers (Figure 6e). To further confirm the role of RhoA in attenuating EMT, we transfected A5RT3 ${ }_{T A K 1}$ cells with RhoA siRNA and observed an increase EMT-like dispersion (Figure 6d). These RhoA-knockdown cells also exhibited changes in the expression of EMT markers (Figure 6e). The inhibition of ROCK and the suppression of RhoA by siRNA in A5RT3 ${ }_{\text {CTRL }}$ also stimulated the expression of EMT markers, albeit at a reduced level (Supplementary Figure S5). Altogether, our observations showed that TAK1 deficiency increased integrin:Rac1-induced ROS, which negatively regulated RhoA by LMW-PTP to accelerate EMT.

\section{Discussion}

Chronic inflammation has been linked to various steps involved in tumorigenesis, including cellular transformation, promotion, invasion and metastasis. ${ }^{22,23}$ Among the numerous cytokines involved in inflammation and tumorigenesis, TGF $\beta 1$ has the broadest effect, influencing cell growth, cancer invasion and spreading. ${ }^{24,25}$ Cellular response to TGF $\beta 1$ is cell-type specific and context-dependent. EMT is a crucial step in tumor progression, and the TGF $\beta$-SMAD signaling pathway as an inductor of EMT in many tumor types is wellrecognized. ${ }^{26,27}$ However, the role of TGF $\beta$-TAK1 signaling in EMT remains unclear. Earlier studies showed that TAK1 through the $\mathrm{NF}_{\kappa} \mathrm{B}$ signaling pathway triggered increased EMT and invasiveness in tumor. ${ }^{28}$ Recent studies also 
a
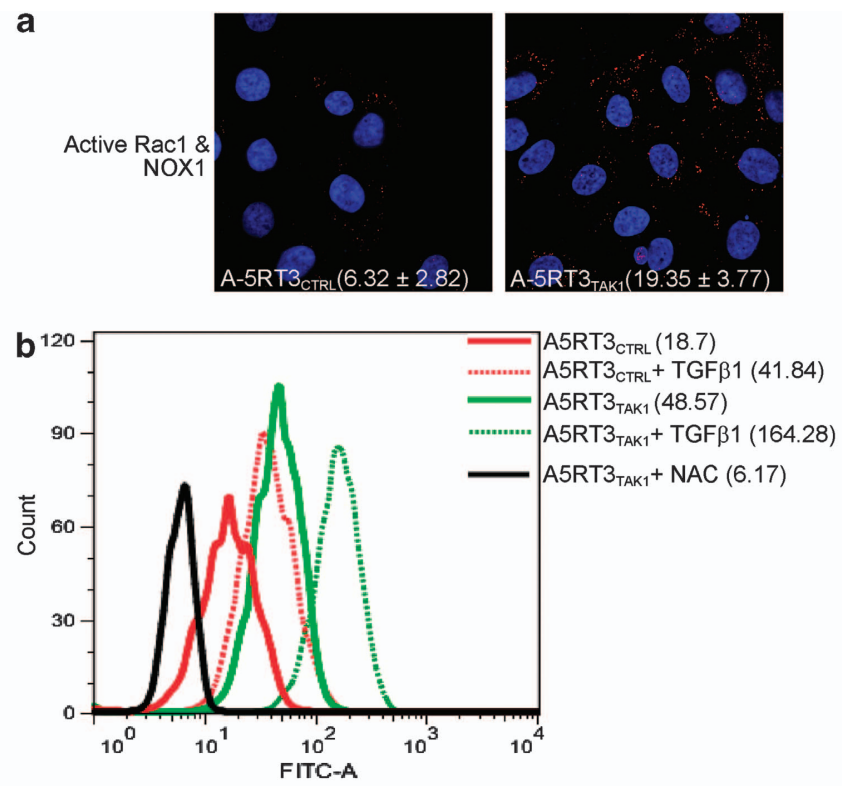

c

TGF $\beta 1(10 \mathrm{ng} / \mathrm{ml})$ for $48 \mathrm{~h}$

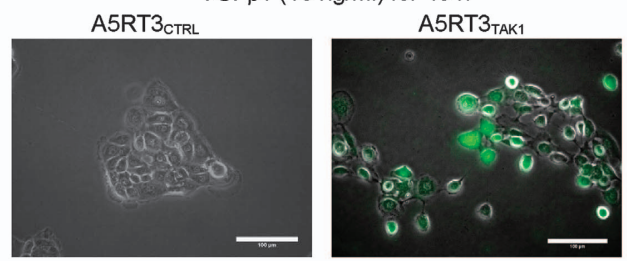

d

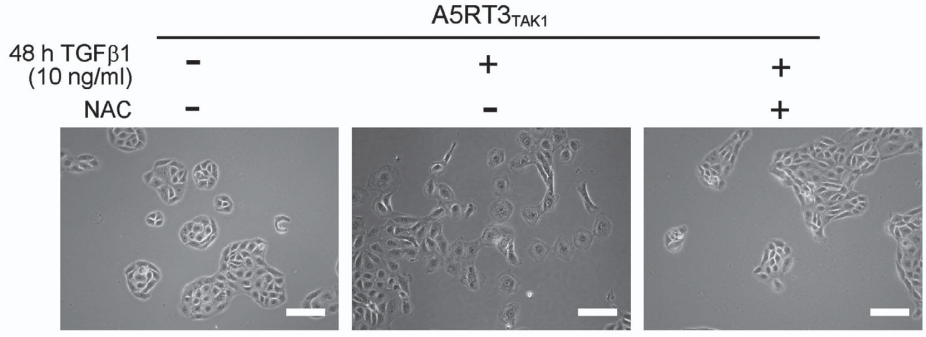

e

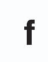

f
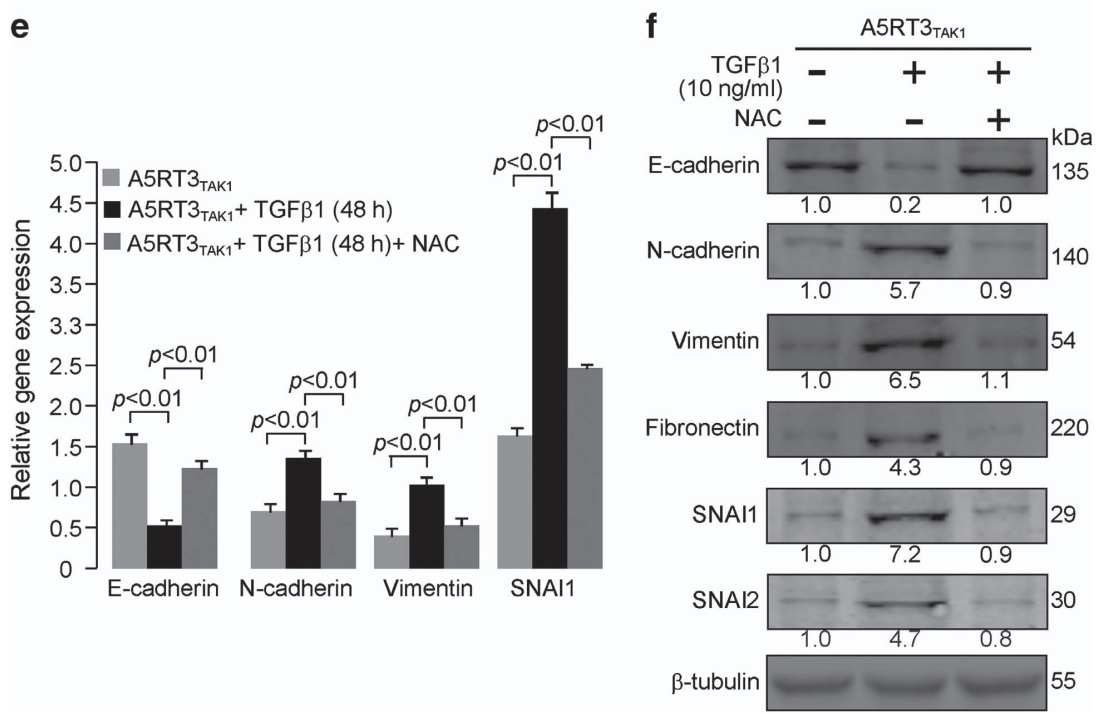
implicated ROS-mediated oxidative stress to promote tumorigenesis ${ }^{29}$ Herein, we found that squamous cell carcinoma progressed earlier into EMT that was conditional on elevated cellular ROS level resulting from TAK1 deficiency (Figure 7). Notably, the expression of TAK1 was consistently reduced in invasive SCCs, but not in benign SCCs, when compared with their cognate perilesional normal tissues. Furthermore, tumors derived from TAK1-deficient cells expressed elevated levels of MMP-9 and laminin-332, the latter was spatially dispersed in the epithelial tumor region. Our investigation further showed that TAK1-deficient cancer cells exhibited an increased cell traction force, one of the earliest cellular response during EMT. ${ }^{30,31}$ This cellular response was associated with an increase in surface integrin $\alpha 5 \beta 1$, a well-known mesenchymal marker in cancer. ${ }^{32}$ We found increased active integrin and active Rac1 interactions in TAK1-deficient cancer cells. The elevated active Rac1 mediated the recruitment of Rac-dependent Nox1 and higher ROS production. Rac1-mediated ROS production resulted in the downregulation of Rho activity in TAK1-deficient cancer cells. The redox-dependent decrease in Rho activity was required for Rac1-induced formation of integrin-mediated cell spreading during TGF $\beta 1$-induced EMT. Conversely, the suppression of Rac1 hindered the progression of EMT by TAK1-deficient cancer cells. The redox-dependent downregulation of RhoA involves the inhibition of LMW-PTP. ${ }^{20}$ Thus, TAK1 modulates Rac1 and Rho GTPases activities, which are critical regulators of actin cytoskeleton remodeling during EMT.

Numerous studies have revealed a biphasic role of epithelial TGF $\beta 1$ signaling in tumorigenesis, but our molecular understanding for this phenomenon remains unclear. In the early stages of cancer, TGF $\beta 1$ has tumor suppressive effects, whereas in the advanced stages of cancer, TGF $\beta 1$ can promote the growth of tumor cells, as well as induce EMT, which results in metastasis. ${ }^{25,33}$ The role of TGF $\beta$-TAK1 in tumorigenesis is only emerging. It was reported that in mouse skin tumor, the deletion of TAK1 gene increases cellular apoptosis due to elevated ROS level. ${ }^{19}$ In fact, TAK1-deficient cancer cells displayed increased propensity to undergo EMT upon TGF $\beta 1$ exposure. Thus, our findings together with that of others suggest that TGF $\beta-$ TAK1 signaling also exhibited a biphasic role during tumor progression. In early development of tumor, TAK1 promotes tumor growth by reining ROS production and ROS-mediated apoptosis. In advance tumor stage, TAK1 discourages EMT and thus delays metastasis. Although we observed a similar increase ROS in our TAK1deficient cancer cells, in contrast to the mouse study, ${ }^{19}$ no significant difference in proliferation or apoptosis were observed. This discrepancy may be due the influence of other surrounding cell types, such as cancer-associated fibroblasts (CAFs). The development and progression of neoplastic epithelia can be understood to arise from the deregulation of this dynamic interaction between normal epithelial and stromal fibroblasts. ${ }^{34,35}$ Recent studies of TGF $\beta$ signaling in stromal fibroblasts showed significant paracrine influence on the behavior of adjacent epithelial cell. Mice with a fibroblastspecific deletion of TGF $\beta$ receptor type II developed intraepithelial neoplasia in prostate and invasive squamous cell carcinoma of the forestomach. ${ }^{36,37}$ Clearly, a better understanding the role of TGF $\beta$-TAK and TGF $\beta$-SMAD signalings in CAFs, and their influence on epithelial cell behaviors requires our future attention. In summary, we showed that TAK1 deficiency increases CTF by a ROS-dependent manner to drive EMT in metastatic A5RT3 cells. Our findings suggest that a dysregulated balance in the activation of TGF $\beta$-TAK1 and TGF $\beta$-SMAD pathways is pivotal for TGF $\beta 1$-induced EMT.

\section{Materials and Methods}

Materials. Antibodies were purchased against integrin $\alpha 5 \beta 1$ (Millipore, Billerica, MA, USA), TAK1, NF $\kappa$ Bp65, phospho NF $\kappa$ Bp65, E-cadherin, SMAD3, phospho-SMAD3, ERK, phospho-ERK, JNK, phospho-JNK (Cell Signaling, Danvers, MA, USA), Rac1, RhoA (Cytoskeleton Inc), activated RhoA, activated Rac1 (NewEast Biosciences, King of Prussia, PA, USA), vimentin, snail2 (Millipore), laminin-332 (AB14509, Abcam, Cambridge, MA, USA), N-cadherin, $\beta$-tubulin, snail1, fibronectin, Nox1 and secondary HRPconjugated antibodies (Santa Cruz Biotechnology, Dallas, TX, USA) for immunoblotting or immunostaining. Transfection reagent ExGen 500 was purchased from Fermentas. Double-promoter lentivirus-based siRNA vector and pFIV packaging kit were acquired from System Biosciences (Mountain View, CA, USA). Pooled siRNA and transfection reagent were purchased from Dharmacon (Pittsburgh, PA, USA). All restriction enzymes and DNA/ RNA-modifying enzymes were obtained from Fermentas. TGF $\beta 1$ and IL-1 were purchased from Peprotech (Rehovot, Israel). Otherwise, chemicals were purchased from Sigma-Aldrich (St. Louis, MO, USA).

\begin{abstract}
Reverse-transcription and real-time PCR (qPCR). Total RNA was isolated and reverse transcribed as previously described. ${ }^{9}$ The CDNA were used for aPCR using SYBR-Green Master mix (KAPABiosystem, Woburn, MA, USA). Melt curve analysis was included to assure that only one PCR product was formed. Expression was normalized to the control gene ribosomal L27, which did not change under any of the experimental conditions studied. Average fold change was derived from three independent experiments and two-tailed student $t$-tests were performed to evaluate statistical significance of expressional changes. The sequences of primers are in Supplementary Table S1.
\end{abstract}

Tumorigenicity assay. Primary tumor formation was monitored over a period of 2 months after subcutaneous injection of $2 \times 10^{6}$ tumor cells resuspended in $100 \mu \mathrm{l}$ of DMEM medium containing Matrigel $(5 \mathrm{mg} / \mathrm{ml})$ into the interscapular region of 6-week-old BALB/c athymic nude mice. Animals were killed at tumor size of $10 \mathrm{~mm}$ diameter. Excised tumors were fixed in $4 \%$ paraformaldehyde overnight followed by overnight equilibration in 30\% sucrose/PBS for cryoprotection before embedding and freezing in OCT medium (Tissue-tek, Alphen aan den Rijn, The Netherlands). Frozen tumor tissues were sectioned and immunostained as previously described. ${ }^{38}$

Figure 5 Role of elevated ROS during TGF $\beta 1$-induced EMT in A5RT3 TAK1. $_{\text {. }}$ (a) PLA assay of active Rac1 and Nox1 in A5RT3 $3_{\text {CTRL }}$ and A5RT3 $3_{\text {TAK1 }}$ cell. Representative images were shown with mean number of PLA spots per nucleus \pm S.D. indicated; $n=3$. (b) A5RT3 $3_{C T R L}$ and A5RT3 $3_{\text {TAK1 }}$ with or without $48 \mathrm{~h}$ of TGF $\beta 1$ (10 $\mathrm{ng} / \mathrm{ml}$ ) treatment were stained with DCF and analyzed with flow cytometry. Antioxidant NAC-treated cells $(100 \mu \mathrm{M})$ served as a negative control. Image shown is representative of three different experiments. Values shown indicate mean fluorescence intensity. (c) Phase-contrast images showing DCF staining of A5RT3CTRL and A5RT3 TAK1 after $48 \mathrm{~h}$ of TGF $\beta 1$ $(10 \mathrm{ng} / \mathrm{ml})$ treatment. Scale bar, $100 \mu \mathrm{m}$. (d) Phase-contrast images A5RT3 TAK1 cells subjected to indicated treatements. NAC (100 $\mu \mathrm{M})$ was used to quench ROS. Scale bar, $100 \mu \mathrm{m}$. (e) qPCR analysis of EMT markers in TGF $\beta 1$ induced A5RT3 TAK $1_{1}$ with NAC treatment. Samples were normalized with reference gene, L27. (f) Representative blots of EMT markers in TGF $\beta 1$ induced A5RT3 $3_{\text {TAK } 1}$ with NAC treatment were shown with densitometry values indicated below respective lanes. Samples were normalized with tubulin as a loading control. Data represent means \pm S.D.; $n=3$ 
a

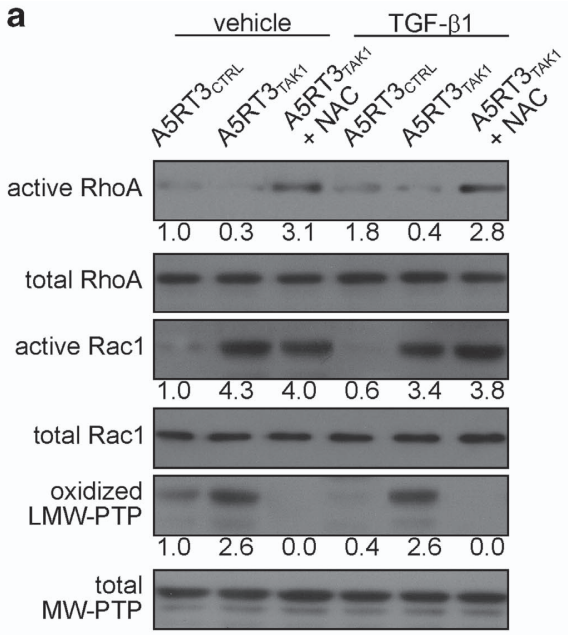

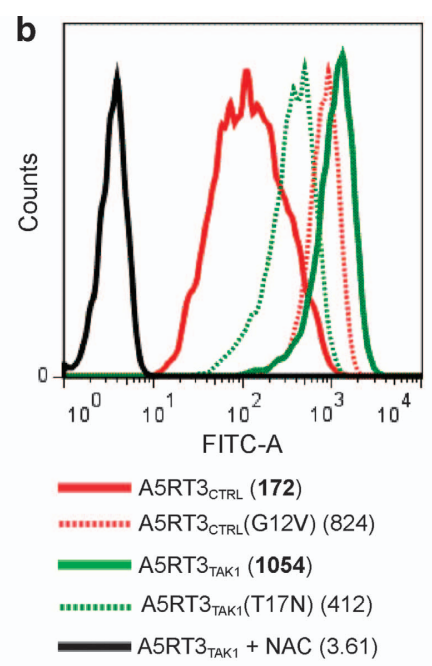

C
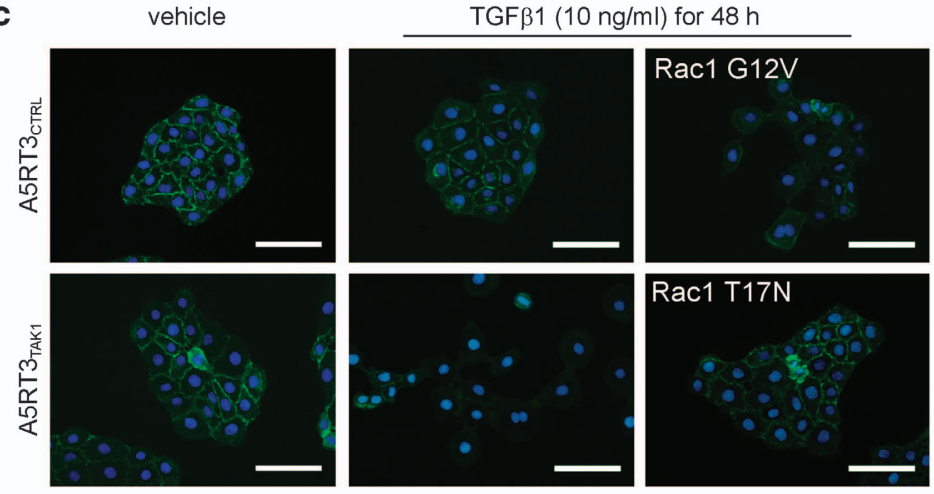

d

A5RT3 TAK1 $_{1}$

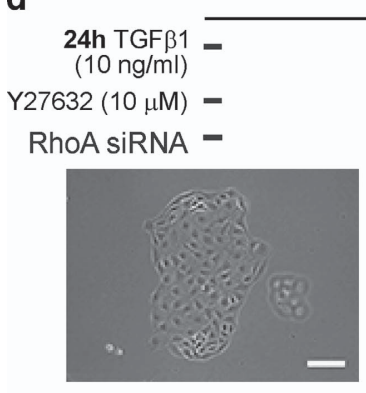

e

$\mathrm{A} 5 \mathrm{RT} 3_{\mathrm{CTRL}}$

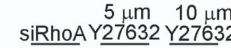
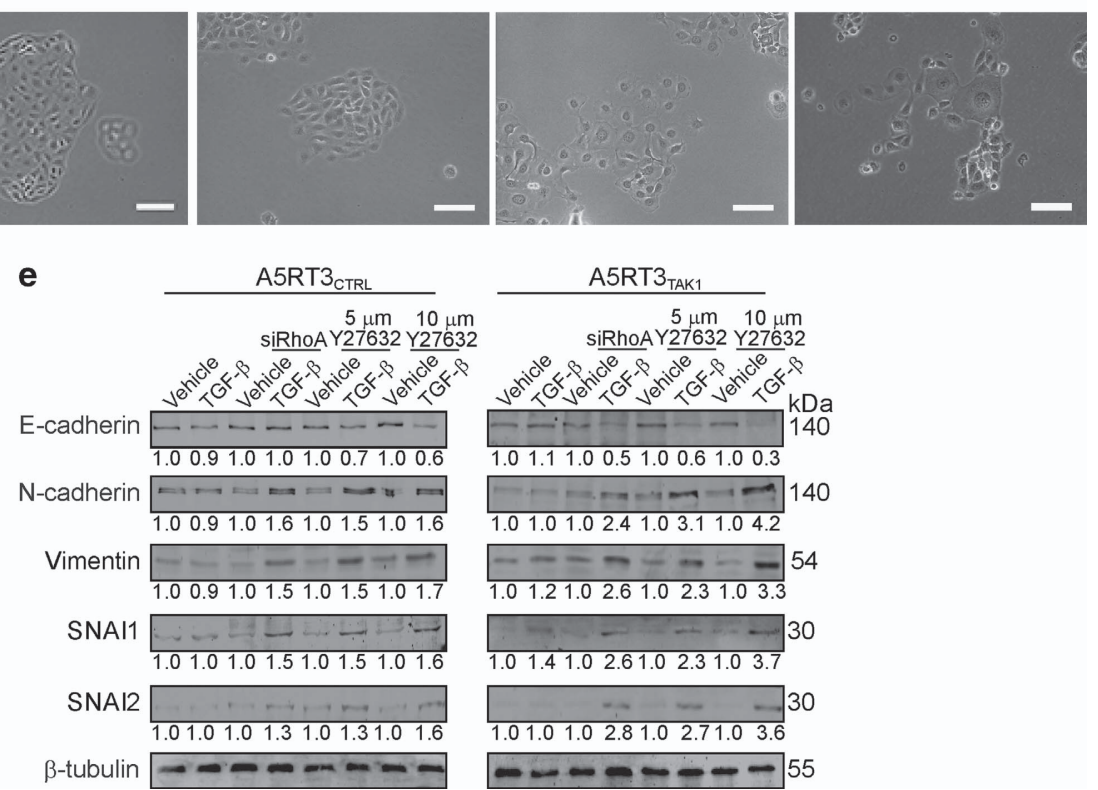
$\mathrm{A} \mathrm{RT} 3_{\text {TAK }}$

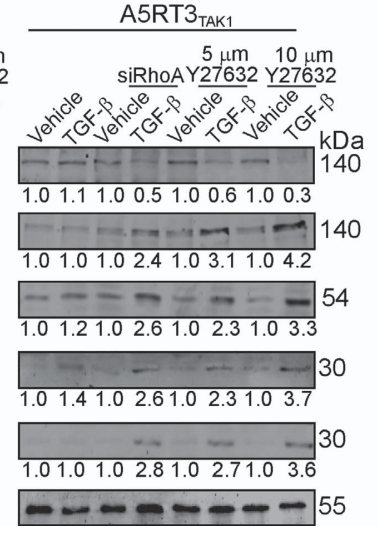


Immunostaining and proximity ligation assay. Cells or tissue sections were fixed with $4 \%$ paraformaldehyde for $15 \mathrm{~min}$, blocked and permeabilized in $3 \%$ BSA with $0.05 \%$ Triton-X for $1 \mathrm{~h}$. Cells or tissue sections were incubated with either anti-vinculin $(1: 100)$, or monoclonal anti-E- cadherin $(1: 25)$, or anti-N-cadherin $(1: 25)$ or in anti-laminin-332 $(1: 50)$ in $0.3 \%$ BSA overnight at $4{ }^{\circ} \mathrm{C}$. Secondary goat anti-rabbit Alexa Fluor 488 antibodies (1:200) in $0.2 \%$ BSA were added with incubation for $1 \mathrm{~h}$ at room temperature in the dark. Cells were then incubated with 1:50 dilution of Alexa Fluor 594 phalloidin (Molecular Probes, Eugene, OR, USA) in 2\% BSA for $2 \mathrm{~h}$ and counterstained with DAPI (Vectashield, Burlingame, CA, USA). Between each step, slides were washed thrice with PBS. Immunofluorescent and phase-contrast images were taken using Nikon 801 Eclipse microscope equipped with a Nikon DS-SI1 camera, using a (20x/0.5) DIC objective and the NIS element D3.0 software with constant exposure and gain. Respective PLA assays were carried out as per manufacturer protocol (Olink Bioscience, St. Louis, MO, USA). Images were taken using Carl Zeiss (Thornwood, NY,

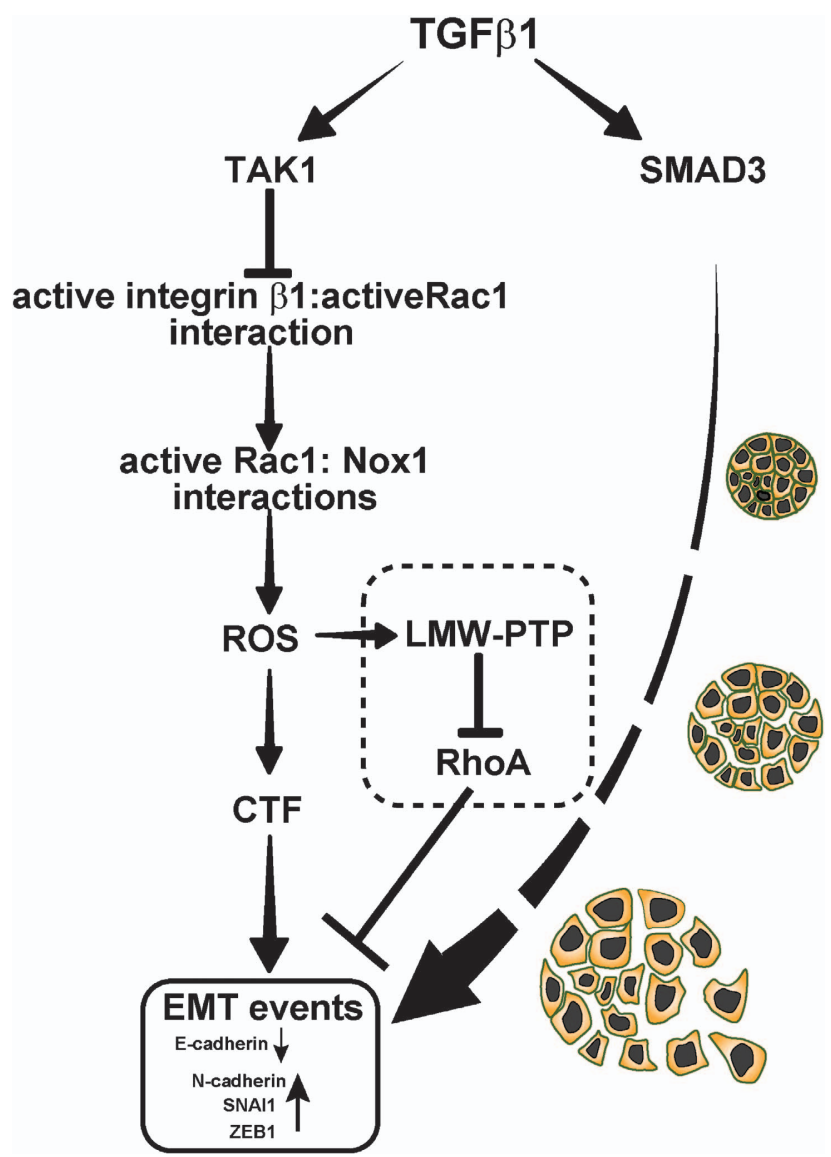

Figure 7 Proposed mechanism by which TAK1 inhibits TGF $\beta 1$-induced EMT. TAK1 likely counters the well-established TGF $\beta 1$-SMAD3 induction of EMT with the suppression of integrin expression and hence Rac1 activation. Rac activity otherwise promotes ROS production via the recruitment of Nox1, which oxidizes LMW-PTP and correspondingly inhibit RhoA activity, promoting EMT. Elevated ROS also leads to increased CTF, a characteristic of EMT progression
USA) confocal microscope LSM 710meta using a Plan-Apochromat $\times 63 / 1.4$ oil DIC objective, and ZEN 2009 LE software with constant exposure and gain. The resulting fluorescent spots were quantified using Duolink ImageTool (Olink Bioscience).

Flow cytometry. Cells were seeded and grown on $75 \mathrm{~cm}^{2}$ culture flask (Corning, Tewksbury, MA, USA) until $50 \%$ confluence. Cells were then washed with PBS and incubated with $25 \mathrm{mM}$ EDTA/PBS for $20 \mathrm{~min}$. EDTA suspended cells were then spun down at $250 \times \mathrm{g}$ and fixed with resuspension in $4 \%$ paraformaldehyde for $20 \mathrm{~min}$ at room temperature. Fixed cells were spun down at 700 r.p.m. and washed with PBS twice through resuspension. Washed cells were then treated with anti- $\beta 1, \beta 3, \beta 4, \beta 5, \beta 7, \alpha 5 \beta 1$ integrin antibodies, respectively at concentration $(1: 25)$ in $10 \%$ BSA and incubated at room temperature for $1 \mathrm{~h}$. Cells were washed twice with PBS resuspension before incubation in secondary antibody conjugated with Alexa Fluor 488 at concentration $(1: 100)$ in 10\% BSA at room temperature in the dark for $1 \mathrm{~h}$. Stained cells were analyzed with Becton Dickinson (BD, Franklin Lakes, NJ, USA) LSRII flow cytometer. Analysis of flow cytometry results were performed with Flowjo software (Ashland, OR, USA). For ROS detection, cells were incubated with $10 \mu \mathrm{M}$ CM-H2DCFDA (Invitrogen, Carlsbad, $\mathrm{CA}$, USA) for $30 \mathrm{~min}$ at $37^{\circ} \mathrm{C}$ prior to trypsinization and analysis by flow cytometry (LSRII from BD Biosciences). Results were analyzed by the Flowjo software. ROS was quenched with the addition of $\mathrm{N}$-acetyl-L-cysteine (NAC) to a final concentration of $100 \mu \mathrm{M}$. CM-H2DCFDA-labeled cells were also imaged with the Nikon 80l Eclipse microscope equipped with a Nikon DS-SI1 camera, using a $(\times 20 / 0.5)$ DIC objective and the NIS element D3.0 software with constant exposure and gain.

Western blot analysis and detection of oxidized proteins. Cells $\left(5 \times 10^{5}\right)$ were lyzed in $100 \mu$ lysis buffer $\left(50 \mathrm{mM} \mathrm{Na}_{2} \mathrm{H}_{2} \mathrm{PO}_{4}, 250 \mathrm{mM} \mathrm{NaCl}, 1 \%\right.$ Triton-X and $0.1 \%$ SDS). Equal amount of proteins were resolved by SDS-PAGE and transferred onto PVDF membrane (Millipore). Immunoblotting for $\beta$-tubulin was used to ensure equal loading of total proteins. The primary antibodies were used at dilutions of 1:4000 (TAK1), 1:2000 (NF $\kappa$ Bp65), 1:2000 (phospho NF $\kappa$ Bp65), 1:1000 (SMAD 3), 1:2000 (Fibronectin), 1:1000 (Vimentin) and $1: 5000$ ( $\beta$-tubulin). Secondary antibodies were used at 1:5000 (anti-rabbit lgG), $1: 10000$ (anti-mouse $\operatorname{lgG}$ ) and 1:5000 (anti-goat lgG). All incubations were performed in $1 \times \mathrm{TBS}, \mathrm{pH} 7.5$, with $0.1 \%$ Tween-20 and either $5 \%$ fat-free milk ( $\beta$-tubulin) or $5 \%$ bovine serum albumin (TAK1). Washing was performed with the same buffer without milk or BSA. The procedure to identify oxidized proteins (i.e. LMW-PTP) with biotin-NM was performed as earlier described. ${ }^{39}$ Densitometry measurements of bands were performed with ImageJ software. The first band in each blot was given an arbitrary density of 1 , and relative densities of the remaining bands were determined. Densitometry results of all individual bands are divided by those from tubulin and the ratio is presented as values below individual lanes.

Cell culture and transient transfection assay. Human embryonic kidney (HEK293-T) and human skin squamous cell carcinoma (A5RT3) cells were routinely maintained in DMEM and FBS (Hyclone, Pittsburgh, PA, USA). Transfection of $A 5 R T 3_{\text {CTRL }}$ and $A 5 R T 3_{\text {TAK1 }}$ cells was carried out as per manufacturer's protocol (Fugene, Madison, WI, USA) with either plasmids expressing constitutive-active Rac1 G12V or dominant-negative Rac T17N.

Cell traction force (CTF) measurements. To measure cell contraction forces, cells were cultured on PDMS substrate embedded with fluorescent beads. Briefly, glass coverslips were cleaned by use of oxygen plasma before it was placed in a vacuum desiccator containing a small drop of $1 \mathrm{H}, 1 \mathrm{H}, 2 \mathrm{H}, 2 \mathrm{H}$-perfluorooctyltrichlorosilane for $30 \mathrm{~min}$. Following which, the surface-treated coverslips were removed and $100 \mu \mathrm{l}$ of diluted yellow-green FluoSpheres microspheres $(0.2 \mu \mathrm{m}$, carboxylate-modified, $0.1 \mathrm{vol} \%$,

Figure 6 Redox regulation of RhoA by Rac1 facilitates TGF $\beta 1$ induced EMT. (a) RhoA and Rac1 activities and LMW-PTP oxidation were compared with immunoblotting as respectively indicated. $10 \mathrm{ng} / \mathrm{ml} \mathrm{TGF} \beta 1$ and $100 \mu \mathrm{M}$ of antioxidant NAC were used respectively for treatment duration of $48 \mathrm{~h}$. (b) FACS analysis of tumor cells transfected with or without constitutive-active (G12V) and dominant-negative Rac1 (T17N). Antioxidant NAC-treated cells $(100 \mu \mathrm{M})$ served as a negative control. Values shown indicate mean fluorescence intensity. (c) Representative images of E-cadherin (green/488 nm) immunostaining of tumor cells with respectively indicated treatments and transfection. Cell nucleus is stained with DAPI (blue). Scale bar, $100 \mu \mathrm{m}$. (d) Phase-contrast images of A5RT3 TAK1 $_{1}$ cells with and without TGF $\beta 1$ induction were inhibited with Y27632 $(10 \mu \mathrm{M})$ for $24 \mathrm{~h}$ or transfected with siRNA for RhoA. Representative phase-contrast images of treated cells. Scale bar, $100 \mu \mathrm{m}$. (e) Representative blots of EMT markers in TGF $\beta 1$-induced A5RT3 ${ }_{\text {CTRL }}$ and A5RT3 TAK1 $_{1}$ treated with ROCK Y27632 or transfected with pooled siRNA for RhoA. Values shown with densitometry values indicated below respective lanes. Samples were normalized with tubulin as a loading control. Data represent means \pm S.D.; $n=3$ 
Molecular Probes) was added to the coverslips with the treated surface facing upwards. This step forms a monolayer of fluorescent beads on the coverslips. Thin film of PDMS were generated by placing $15 \mu \mathrm{l}$ of PDMS mixture (before curing, $1: 60 \mathrm{w} / \mathrm{w}$ of cross-linker to base ratio) on the fluorescent microspheres coated glass coverslips. One layer of Scotch tapes was applied as a spacer before putting the other bare glass coverslip on the top of fluorescent microspheres coated glass coverslips. The PDMS droplet was flattened under the weight of the upper coverslip. The entire assembly was left undisturbed for $5 \mathrm{~min}$ and then placed in $37^{\circ} \mathrm{C}$ incubator overnight before carefully peeling off the upper bonded PDMS thin film with fluorescent microspheres on its top. Only samples with well-dispersed beads were used in the study. Substrates were UV sterilized for $30 \mathrm{~min}$. As PDMS surface does not allow cells to adhere, the substrate was coated by incubation with human plasma derived fibronectin solution $(50 \mu \mathrm{g} / \mathrm{ml}$ in $0.02 \mathrm{M}$ acetic acid for thin coating) overnight to promote cell attachment. Coated substrates were rinsed with PBS, and stored in the dark at $4{ }^{\circ} \mathrm{C}$ prior to culture. $1 \times 10^{3}$ cells/inserts were seeded for $48 \mathrm{~h}$ under cell culture conditions. Cells were subsequently treated with $10 \mathrm{ng} / \mathrm{ml}$ TGF $\beta 1$ for $24 \mathrm{~h}$ before traction force measurement. All experiments were triplicated.

Calculation of CTFs was based on the elastic moduli of PA substrate and fluorescent beads displacements as previously described. ${ }^{40}$ Spatial displacement of the beads was tacked by taking images of the fluorescent beads before and after cell trypsinization. The cell traction mapping was based on the DIC image of cells on PA substrate and the corresponding traction stress mapping in pseudocolor, which indicated regions of traction stresses (dark blue to light pink according to stresses from low to high). Briefly, the traction force at discrete point $\vec{f}_{i}$, located at the position $\left(x_{i}, y_{i}\right)$ was calculated based on the following formulation: $\vec{u}_{t}(x, y)=\sum_{i=1}^{n} \vec{G}\left(x-x_{i}, y-y_{i}\right) \vec{f}_{i}$, where $\vec{G}$ denoted the Greens' tensor and $\vec{u}_{t}$ denoted the experimental displacements of fluorescent beads at position $\left(x_{i}, y_{i}\right)$. The overall force of the cell $(F)$ is an integral of the traction field magnitude over the area, $F=\iint \sqrt{T_{x}^{2}(x, y)+T_{y}^{2}(x, y)} \mathrm{d} x \mathrm{~d} y$, where $T(x, y)=\left[T_{x}(x, y)+T_{y}(x, y)\right]$ is the continuous field of traction vectors defined at any spatial position $(x, y)$ within the cell.

Transwell invasion assay. $5 \times 10^{4}$ cells were dispensed into the top chamber of transwell migration chambers $(8 \mu \mathrm{m}$ Millicell, Billerica, MA, USA; Hanging Cell Culture Inserts) loaded on a 6-well plate and allowed to attach overnight in cell culturing conditions. One thousand microliters of complete medium (supplemented with $10 \mathrm{ng} / \mathrm{ml} \mathrm{TGF-} \beta 1$ ) was added to each of the underlying wells. For control wells, no TGF- $\beta 1$ was supplemented. This construct was then incubated for $48 \mathrm{~h}$. Following incubation, the inserts were washed with PBS and fixed with $1 \%$ glutaraldehyde for $10 \mathrm{~min}$. The inserts were rinsed with PBS and subsequently stained in $0.1 \%$ crystal violet for $25 \mathrm{~min}$. Cells were thoroughly rinsed with PBS after crystal violet staining. Cotton buds were used to swipe the upper surface of the transwell membrane, ensuring thorough removal of unmigrated cells. The inserts were then immersed in a $500 \mu \mathrm{l}$ of $0.5 \%$ Triton-X solution dispensed in a clean 6-well plate and incubated overnight in the dark. The 6-well plate was sealed with parafilm tape to prevent evaporation. Absorbance of the $500 \mu \mathrm{l}$ volume of $0.5 \%$ Triton- $X$ at $595 \mathrm{~nm}$ was then measured using Nanodrop. The absorbance difference for the test and control wells were used as an index for the comparison of cellular migration.

\section{Conflict of Interest}

The authors declare no conflict of interest.

Acknowledgements. This work is supported by research grant from Ministry of Education (MOE 2010-T2-2-009) to NST. A5RT3 cell line was kindly provided by Dr Petra Boukamp of German Cancer Research Center DKFZ.

1. Mehlen P, Puisieux A. Metastasis: a question of life or death. Nat Rev Cancer 2006; 6 : 449-458.

2. Kalluri R, Weinberg RA. The basics of epithelial-mesenchymal transition. J Clin Invest 2009; 119: 1420-1428.
3. Coussens LM, Werb Z. Inflammation and cancer. Nature 2002; 420: 860-867.

4. Ikushima H, Miyazono K. TGFbeta signalling: a complex web in cancer progression. Nat Rev Cancer 2010; 10: 415-424.

5. Zavadil J, Böttinger EP. TGF-beta and epithelial-to-mesenchymal transitions. Oncogene 2005; 24: 5764-5774.

6. Zavadil J, Cermak L, Soto-Nieves N, Böttinger EP. Integration of TGF-beta/Smad and Jagged1/Notch signalling in epithelial-to-mesenchymal transition. EMBO J 2004; 23 : $1155-1165$.

7. Reuter S, Gupta SC, Chaturvedi MM, Aggarwal BB. Oxidative stress, inflammation, and cancer: how are they linked? Free Radic Biol Med 2010; 49: 1603-1616.

8. Toyokuni S, Okamoto K, Yodoi J, Hiai H. Persistent oxidative stress in cancer. FEBS Lett 1995; 358: 1-3

9. Lam CRI, Tan MJ, Tan SH, Tang MBY, Cheung PCF, Tan NS. TAK1 regulates SCF expression to modulate $\mathrm{PKB} \alpha$ activity that protects keratinocytes from ROS-induced apoptosis. Cell Death Differ 2011; 18: 1120-1129.

10. Omori E, Morioka S, Matsumoto K, Ninomiya-Tsuji J. TAK1 regulates reactive oxygen species and cell death in keratinocytes, which is essential for skin integrity. J Biol Chem 2008; 283: 26161-26168.

11. Kim BG, An HJ, Kang S, Choi YP, Gao MQ, Park $\mathrm{H}$ et al. Laminin-332-rich tumor microenvironment for tumor invasion in the interface zone of breast cancer. Am J Pathol 2011; 178: 373-381.

12. Imura J, Uchida $Y$, Nomoto $K$, Ichikawa $K$, Tomita $S$, lijima $T$ et al. Laminin-5 is a biomarker of invasiveness in cervical adenocarcinoma. Diagnostic pathology 2012; 7: 105.

13. Marinkovich MP. Tumour microenvironment: laminin 332 in squamous-cell carcinoma Nat Rev Cancer 2007; 7: 370-380.

14. Hozumi $Y$, Kondo S, Shimoura T, Aso K. Human squamous cell carcinoma from skin: establishment and characterization of a new cell line (HSC-5). J Dermatol 1990; 17: 143-148.

15. Mueller MM, Peter W, Mappes M, Huelsen A, Steinbauer H, Boukamp P et al. Tumor progression of skin carcinoma cells in vivo promoted by clonal selection, mutagenesis, and autocrine growth regulation by granulocyte colony-stimulating factor and granulocytemacrophage colony-stimulating factor. Am J Pathol 2001; 159: 1567-1579.

16. Motoyama $\mathrm{T}$, Hojo $\mathrm{H}$, Watanabe $\mathrm{H}$. Comparison of seven cell lines derived from human gastric carcinomas. Acta Pathol Jpn 1986; 36: 65-83.

17. Ihara $Y$, Inai $Y$, Ikezaki M. Alteration of integrin-dependent adhesion and signaling in EMT-like MDCK cells established through overexpression of calreticulin. J Cell Biochem 2011; 112: 2518-2528.

18. Maschler S, Wirl G, Spring H, Bredow DV, Sordat I, Beug H et al. Tumor cell invasiveness correlates with changes in integrin expression and localization. Oncogene 2005; 24 : 2032-2041.

19. Omori E, Matsumoto K, Zhu S, Smart RC, Ninomiya-Tsuji J. Ablation of TAK1 upregulates reactive oxygen species and selectively kills tumor cells. Cancer Res 2010; 70: 8417-8425.

20. Nimnual AS, Taylor LJ, Bar-Sagi D. Redox-dependent downregulation of Rho by Rac. Nat Cell Biol 2003; 5: 236-241.

21. Aoudjit F, Vuori K. Integrin signaling in cancer cell survival and chemoresistance. Chemother Res Pract 2012; 2012: 283181.

22. Mantovani A. Cancer: inflammation by remote control. Nature 2005; 435: 752-753.

23. Sethi G, Shanmugam MK, Ramachandran L, Kumar AP, Tergaonkar V. Multifaceted link between cancer and inflammation. Biosci Rep 2012; 32: 1-15.

24. Rahimi RA, Leof EB. TGF-beta signaling: a tale of two responses. J Cell Biochem 2007; 102: 593-608.

25. Meulmeester E, Ten Dijke P. The dynamic roles of TGF- $\beta$ in cancer. J Pathol 2011; 223: 205-218.

26. Brandl M, Seidler B, Haller F, Adamski J, Schmid RM, Saur D et al. IKK( $\alpha$ ) controls canonical TGF( $(3)$-SMAD signaling to regulate genes expressing SNAIL and SLUG during EMT in panc1 cells. J Cell Sci 2010; 123: 4231-4239.

27. Valcourt $\mathrm{U}$, Kowanetz M, Niimi H, Heldin $\mathrm{CH}$, Moustakas A. TGF-beta and the Smad signaling pathway support transcriptomic reprogramming during epithelial-mesenchymal cell transition. Mol Biol Cell 2005; 16: 1987-2002.

28. Huber MA, Azoitei N, Baumann B, Grünert S, Sommer A, Pehamberger $\mathrm{H}$ et al. NF-кB is essential for epithelial-mesenchymal transition and metastasis in a model of breast cancer progression. J Clin Invest 2004; 114: 569-581.

29. Reuter S, Gupta S, Chaturvedi M, Aggarwal B. Oxidative stress, inflammation, and cancer: how are they linked? Free Radic Biol Med 2010; 49: 1603-1616.

30. du Roure O, Saez A, Buguin A, Austin RH, Chavrier P, Silberzan P et al. Force mapping in epithelial cell migration. Proc Natl Acad Sci USA 2005; 102: 2390-2395.

31. de Rooij J, Kerstens A, Danuser G, Schwartz MA, Waterman-Storer CM. Integrin-dependent actomyosin contraction regulates epithelial cell scattering. J Cell Biol 2005; 171: 153-164.

32. Maschler S, Wirl G, Spring H, Bredow D, Sordat I, Beug H et al. Tumor cell invasiveness correlates with changes in integrin expression and localization. Oncogene 2005; 24: 2032-2041.

33. Joshi A, Cao D. TGF-beta signaling, tumor microenvironment and tumor progression: the butterfly effect. Front Biosci 2010; 15: 180-194. 
34. Mueller MM, Fusenig N. Tumor-Associated Fibroblasts and Their Matrix. Springer Verlag, 2011.

35. TIsty TD, Hein PW. Know thy neighbor: stromal cells can contribute oncogenic signals. Curr Opin Genet Dev 2001; 11: 54-59.

36. Bhowmick NA, Chytil A, Plieth D, Gorska AE, Dumont N, Shappell S et al. TGF-beta signaling in fibroblasts modulates the oncogenic potential of adjacent epithelia. Science 2004; 303: 848-851.

37. Cheng N, Bhowmick NA, Chytil A, Gorksa AE, Brown KA, Muraoka R et al. Loss of TGF-beta type II receptor in fibroblasts promotes mammary carcinoma growth and invasion through upregulation of TGF-alpha-, MSP- and HGF-mediated signaling networks. Oncogene 2005; 24: 5053-5068.

38. Zhu P, Tan MJ, Huang RL, Tan CK, Chong HC, Pal M et al. Angiopoietin-like 4 protein elevates the prosurvival intracellular $\mathrm{O} 2(-): \mathrm{H} 2 \mathrm{O} 2$ ratio and confers anoikis resistance to tumors. Cancer Cell 2011; 19: 401-415.
39. Song BJ, Suh SK, Moon KH. A simple method to systematically study oxidatively modified proteins in biological samples and its applications. Methods Enzymol 2010; 473: 251-264.

40. Dembo M, Wang YL. Stresses at the cell-to-substrate interface during locomotion of fibroblasts. Biophys J 1999; 76: 2307-2316.

(c) (i) $\Theta($ Cell Death and Disease is an open-access journal published by Nature Publishing Group. This work is licensed under a Creative Commons Attribution-NonCommercialNoDerivs 3.0 Unported License. To view a copy of this license, visit http://creativecommons.org/licenses/by-nc-nd/3.0/

Supplementary Information accompanies this paper on Cell Death and Disease website (http://www.nature.com/cddis) 

Loss of TAK1 increases cell traction force in a ROS-dependent manner to drive epithelialmesenchymal transition of cancer cells.

Chee Ren Ivan Lam ${ }^{\mathrm{a}}$, Carol Tan ${ }^{\mathrm{a}}$, Ziqiang Teo ${ }^{\mathrm{a}}$, Chor Yong Tay ${ }^{\mathrm{a}}$, Terri Phua ${ }^{\mathrm{a}}$, YunLong Wu ${ }^{\mathrm{b}, \mathrm{c}}$, Ping Qiang Cai ${ }^{\mathrm{b}}$, Lay Poh Tan ${ }^{\mathrm{b}}$, Xiao Dong Chen ${ }^{\mathrm{b}}$, Pengcheng Zhu ${ }^{\mathrm{a}}$, Nguan Soon Tan ${ }^{\mathrm{a}, \mathrm{d}, 1}$

${ }^{a}$ School of Biological Sciences, Nanyang Technological University, 60 Nanyang Drive, Singapore 637551.

${ }^{\mathrm{b}}$ School of Materials Science and Engineering, Nanyang Technological University, 50 Nanyang Avenue, Singapore 639798.

${ }^{\mathrm{c}}$ School of Pharmaceutical Science, Xiamen University, China 361000

${ }^{\mathrm{d}}$ Institute of Molecular and Cell Biology, 61 Biopolis Drive, Proteos, Singapore 138673.

Running title: TAK1 modulates epithelial-mesenchymal transition.

${ }^{1}$ Corresponding author: N.S.T.: Email: nstan@ntu.edu.sg or nstan@imcb.a-star.edu.sg; Tel: +65-63162941; Fax: +65-67913856. 
Figure S1
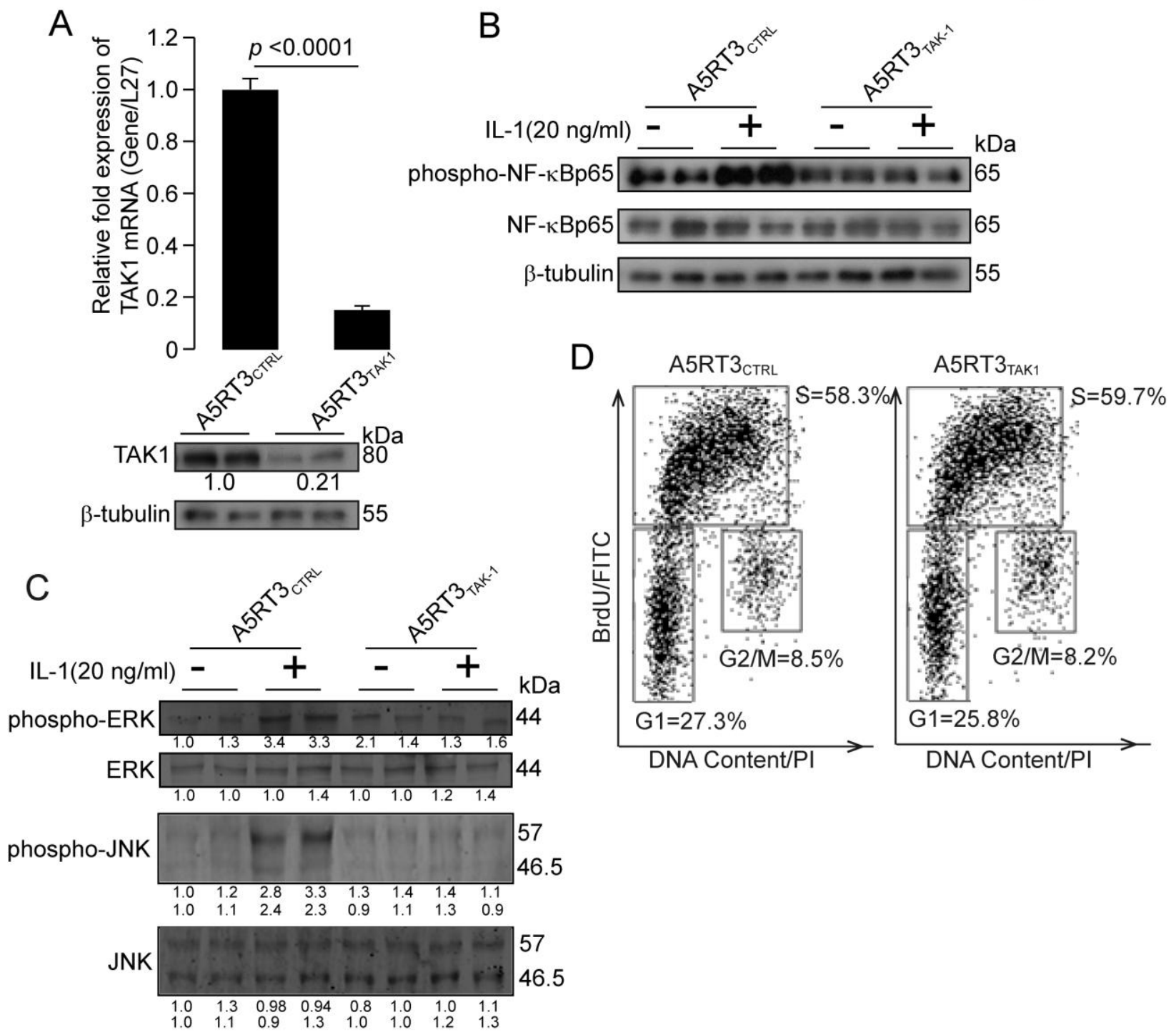

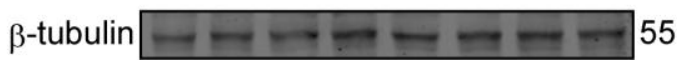


Figure S1

E

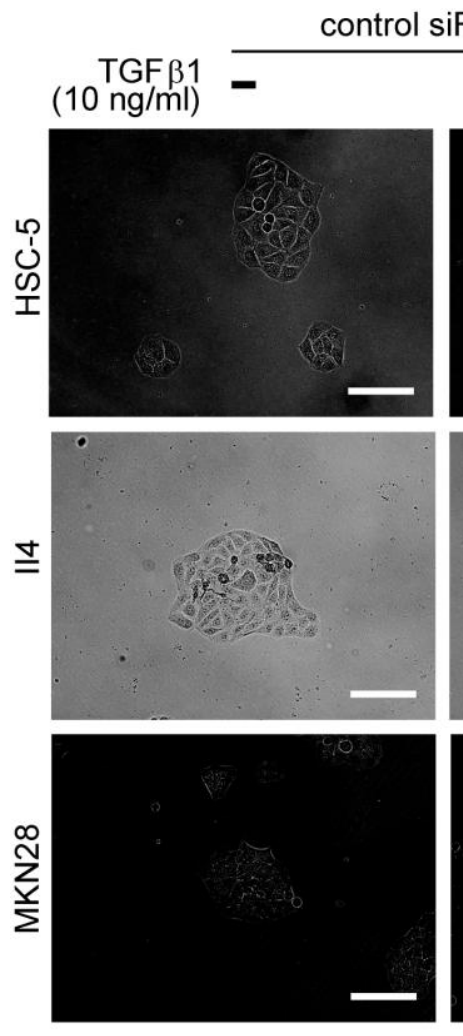

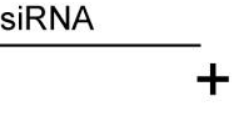
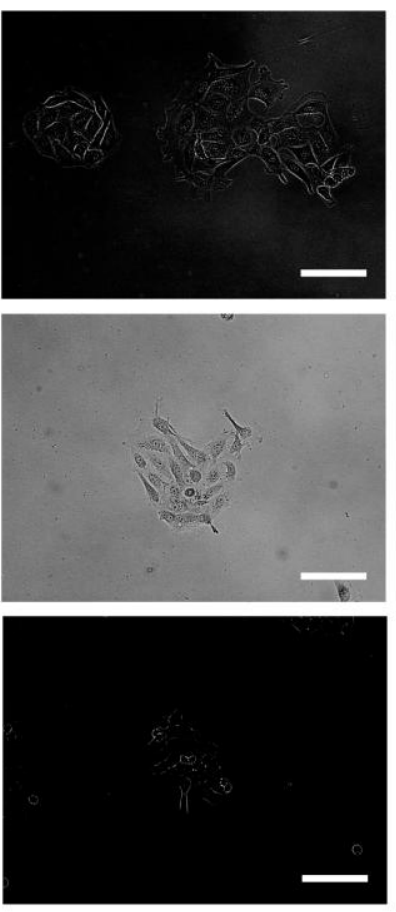
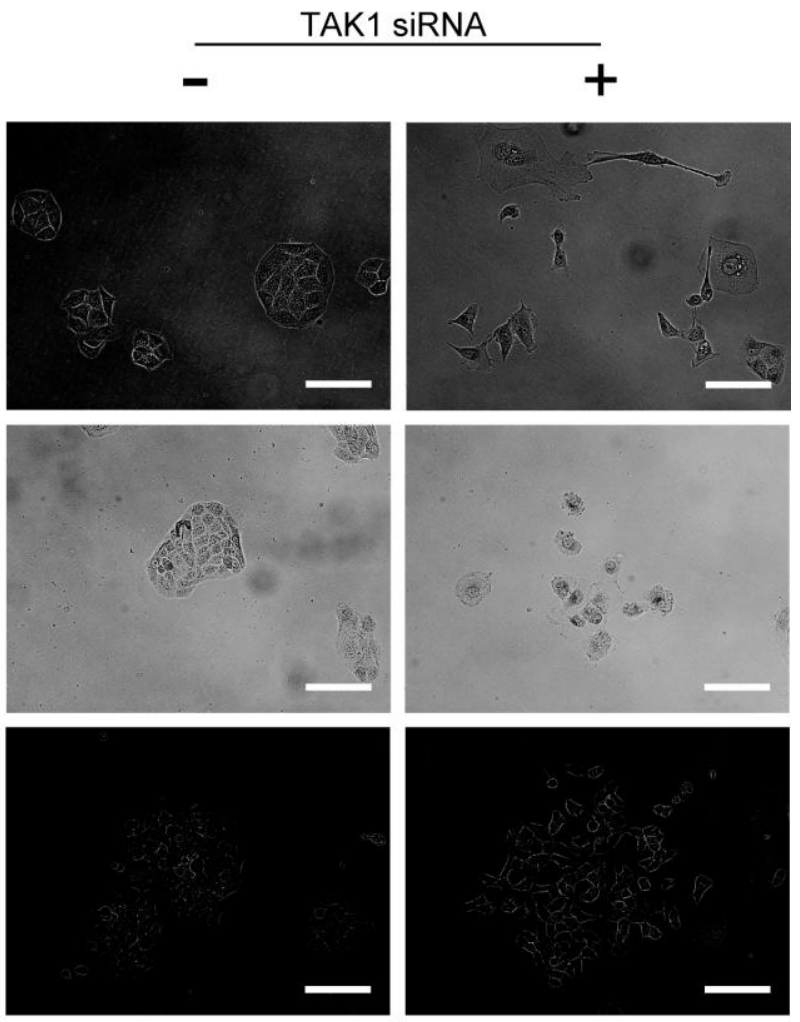

114

F

HSC-5
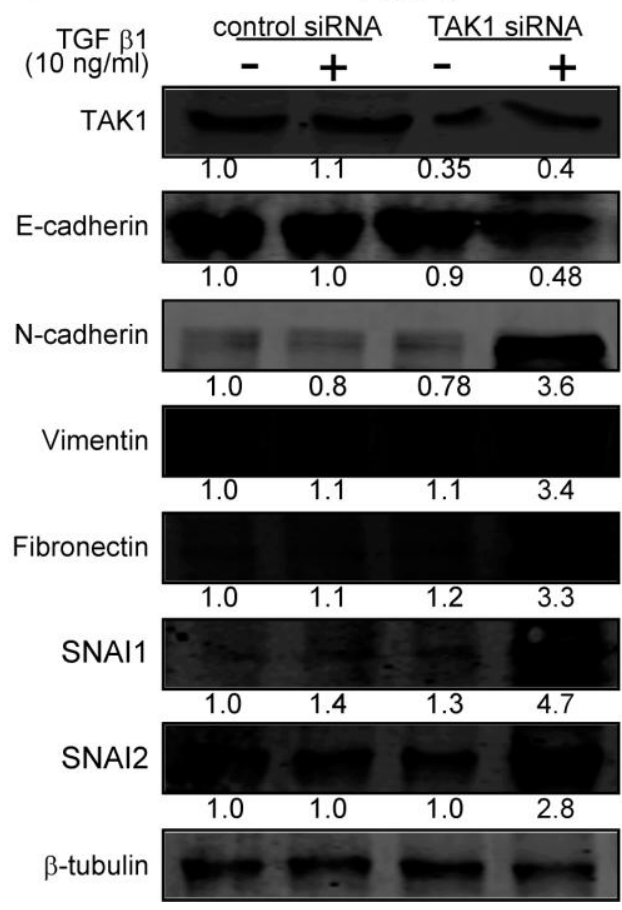

control siRNA TAK1 siRNA
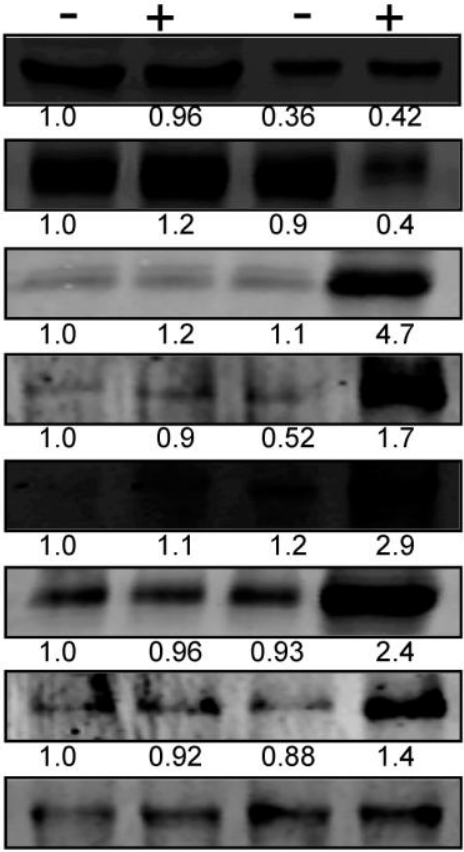

MKN28
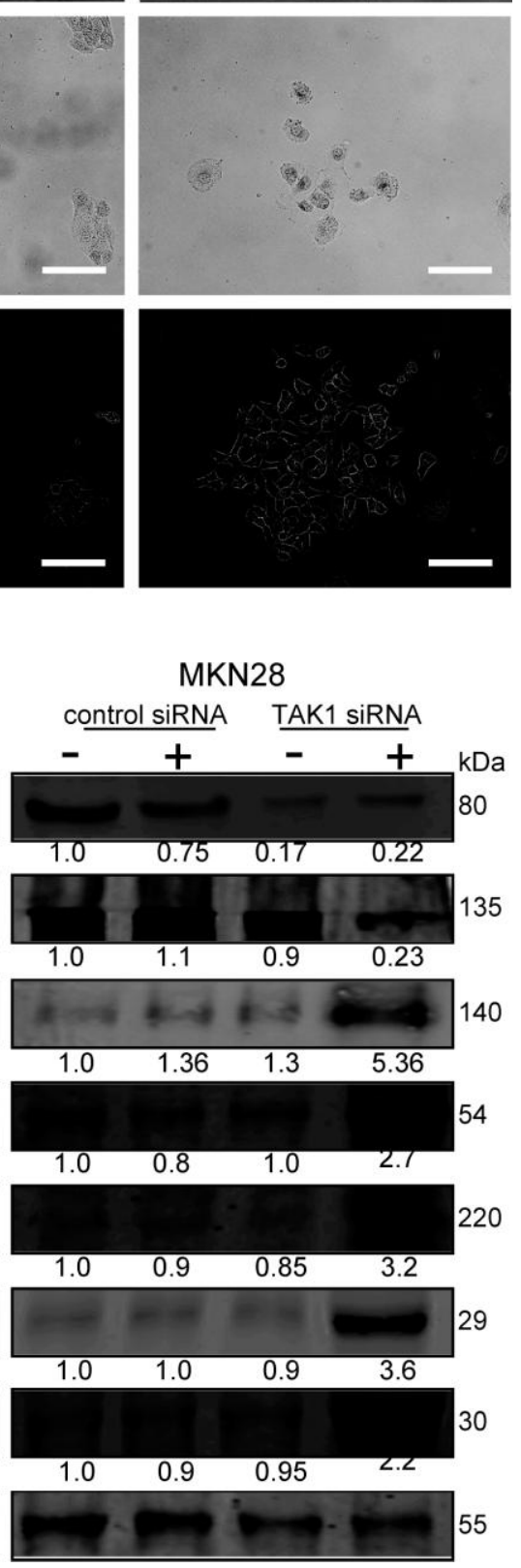
A

Figure S2

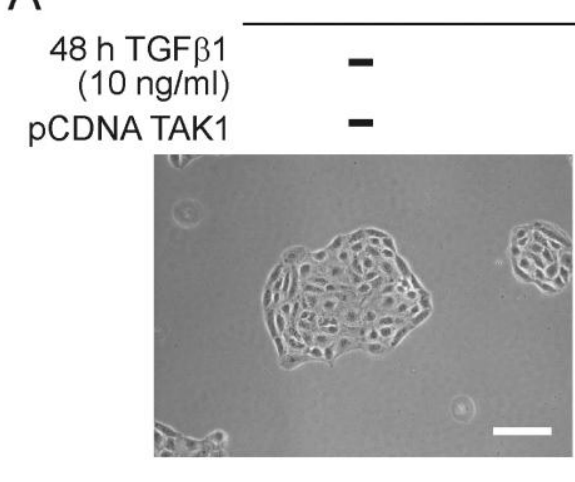

A5RT3 ${ }_{\text {TAK1 }}$

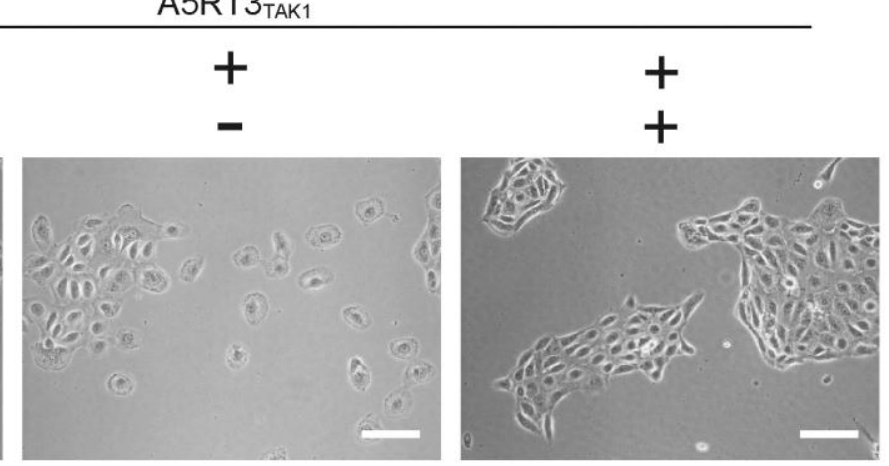

B

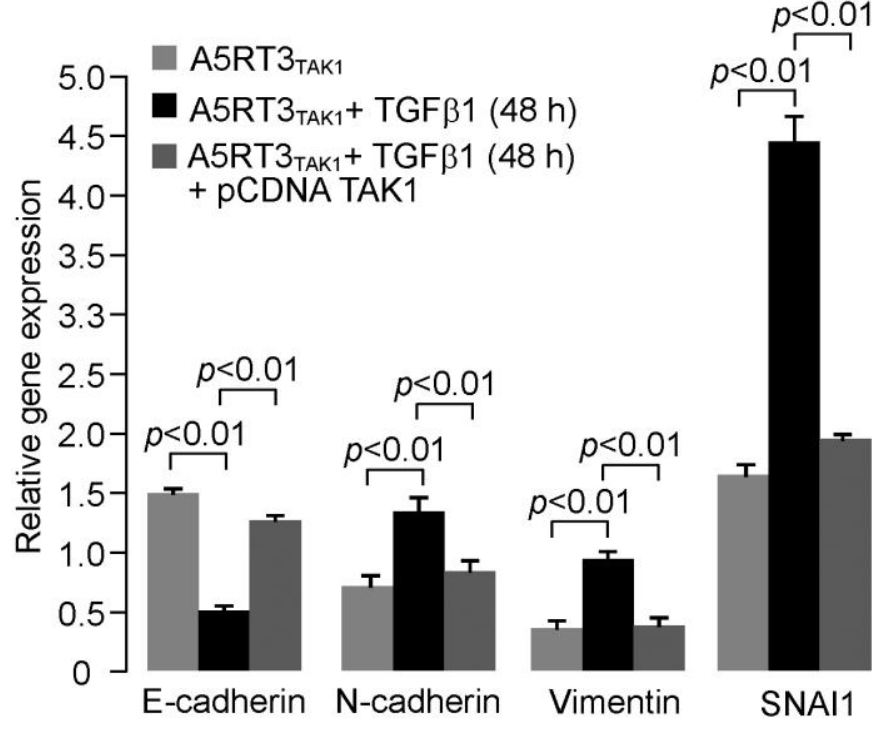

C
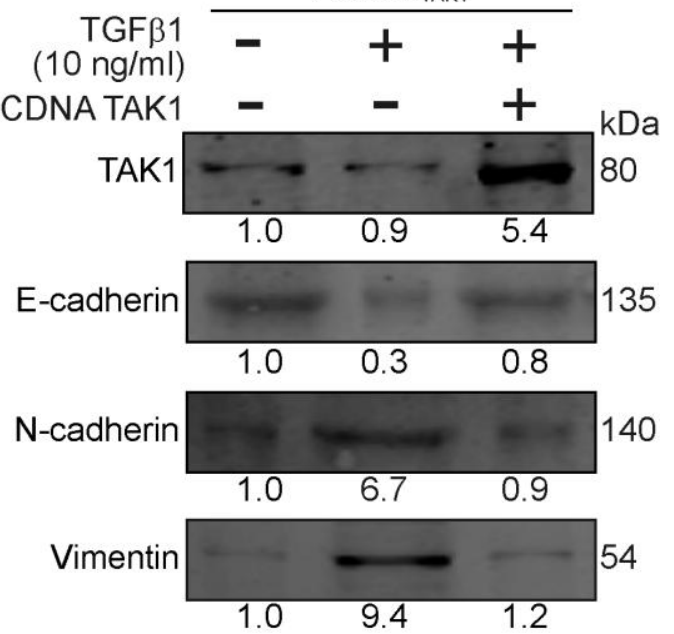

Fibronectin \begin{tabular}{llll}
\cline { 3 - 4 } 2 & & & \\
\cline { 2 - 3 } & 1.0 & 8.4 & 0.9
\end{tabular}
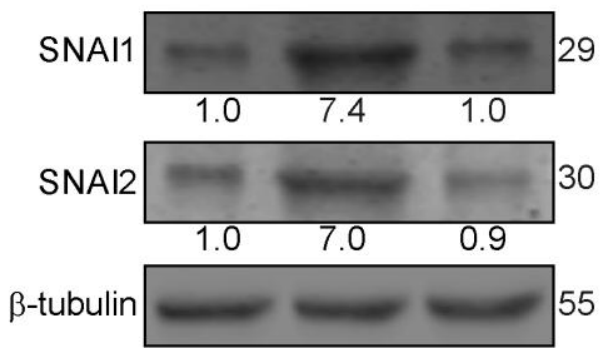
Figure S3

A

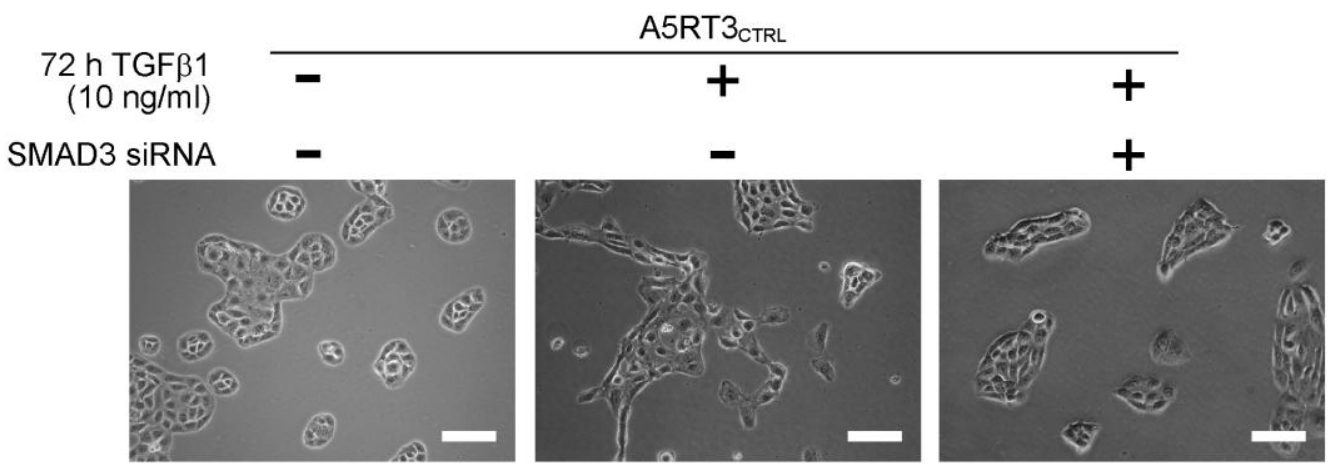

B

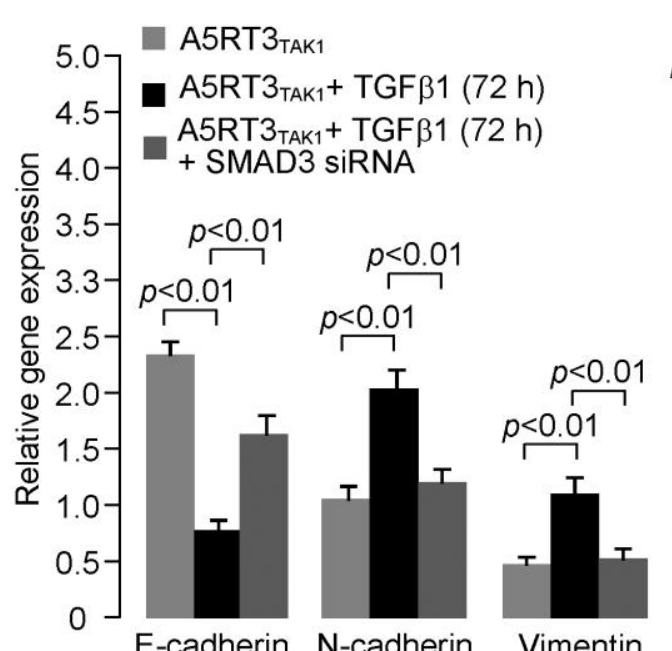

D

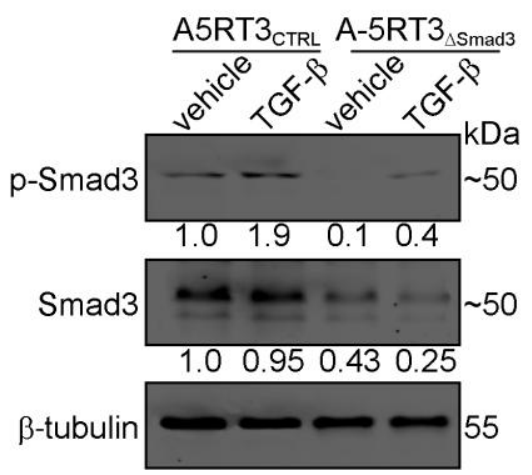

C

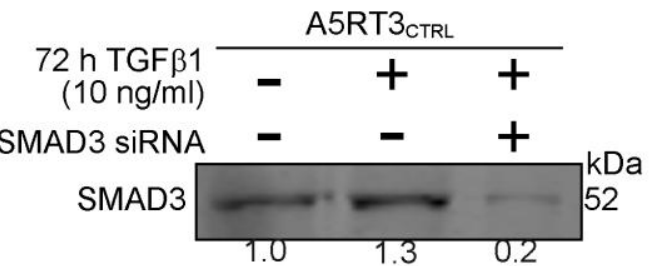

E-cadherin
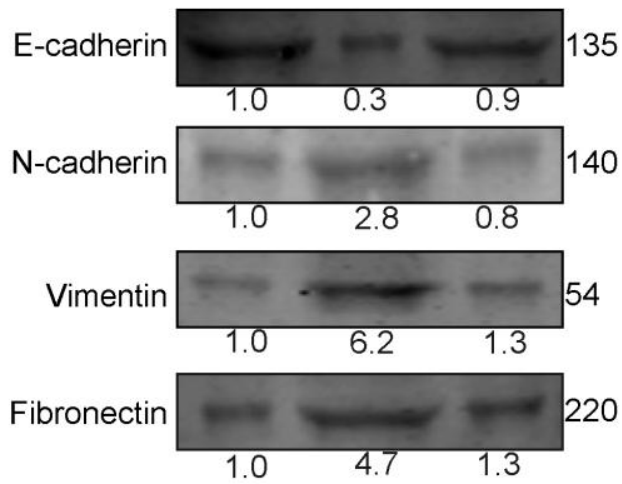

SNAI1

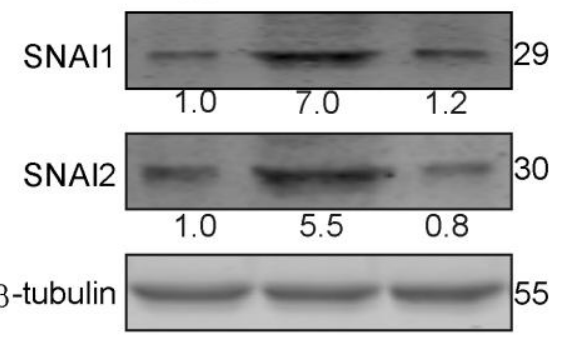


A

A5RT3 $3_{\text {TAK1 }}$

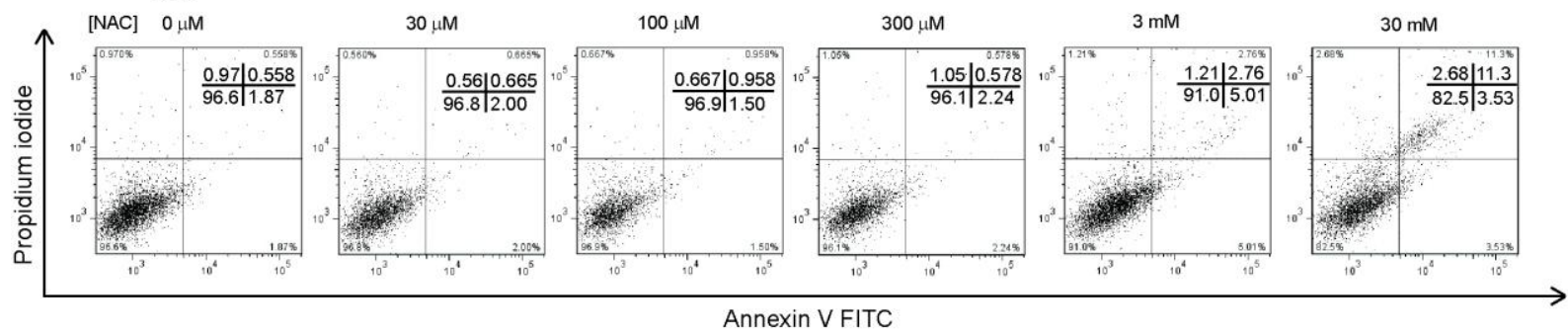

B

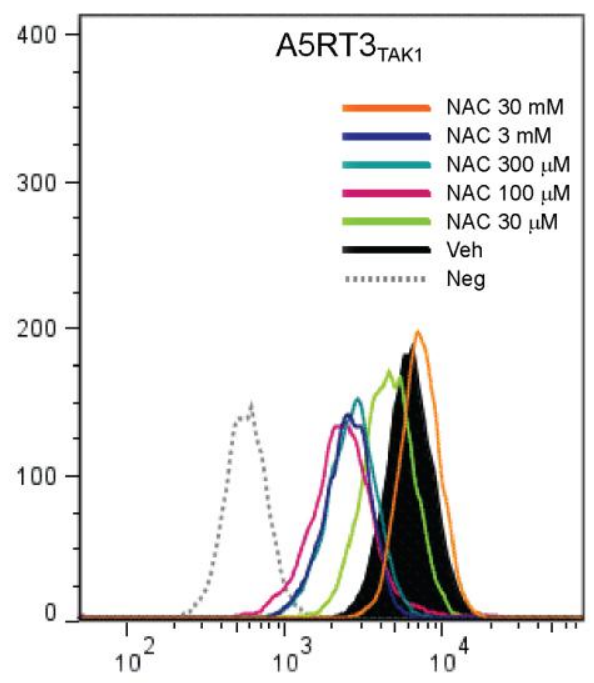

Figure S4

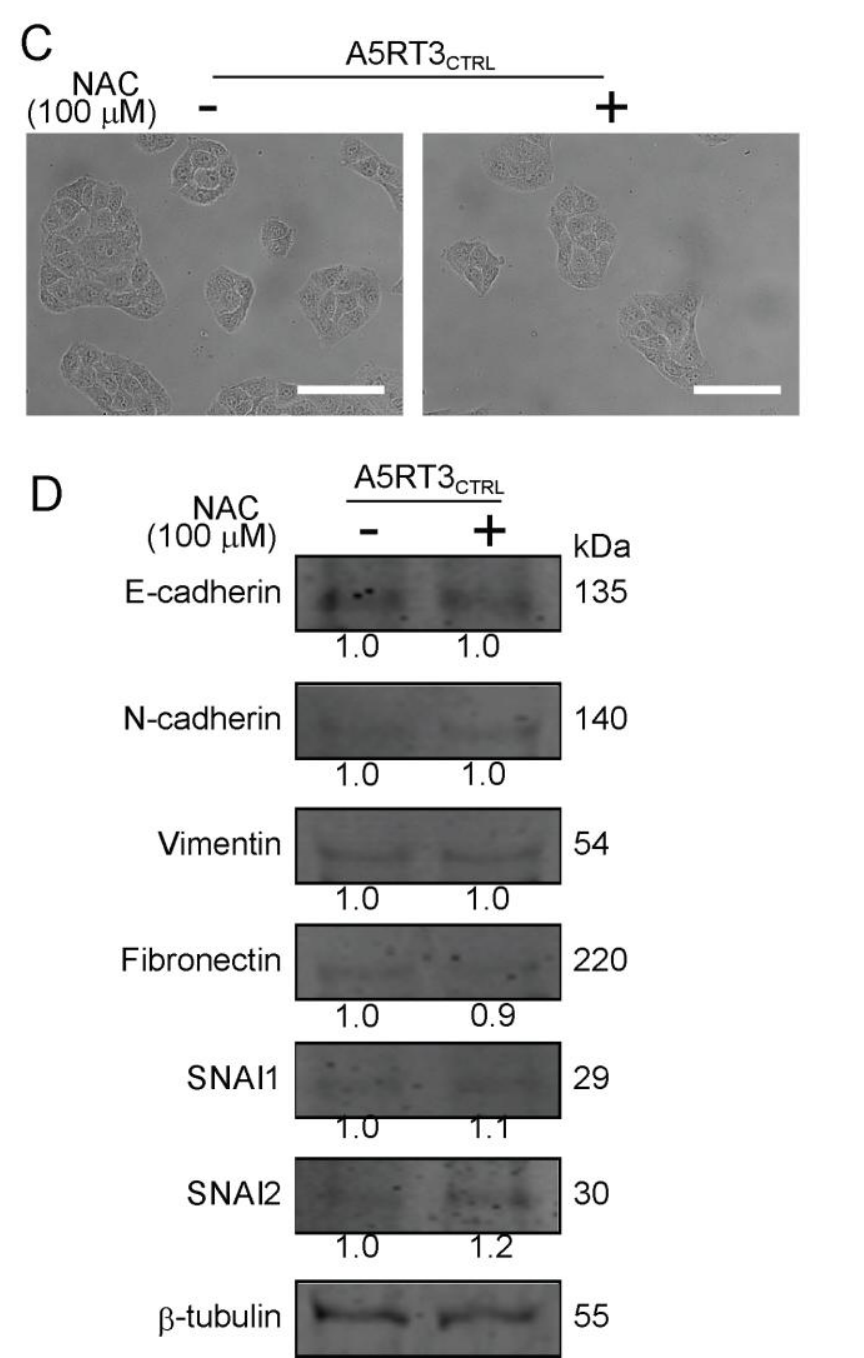


Figure S5

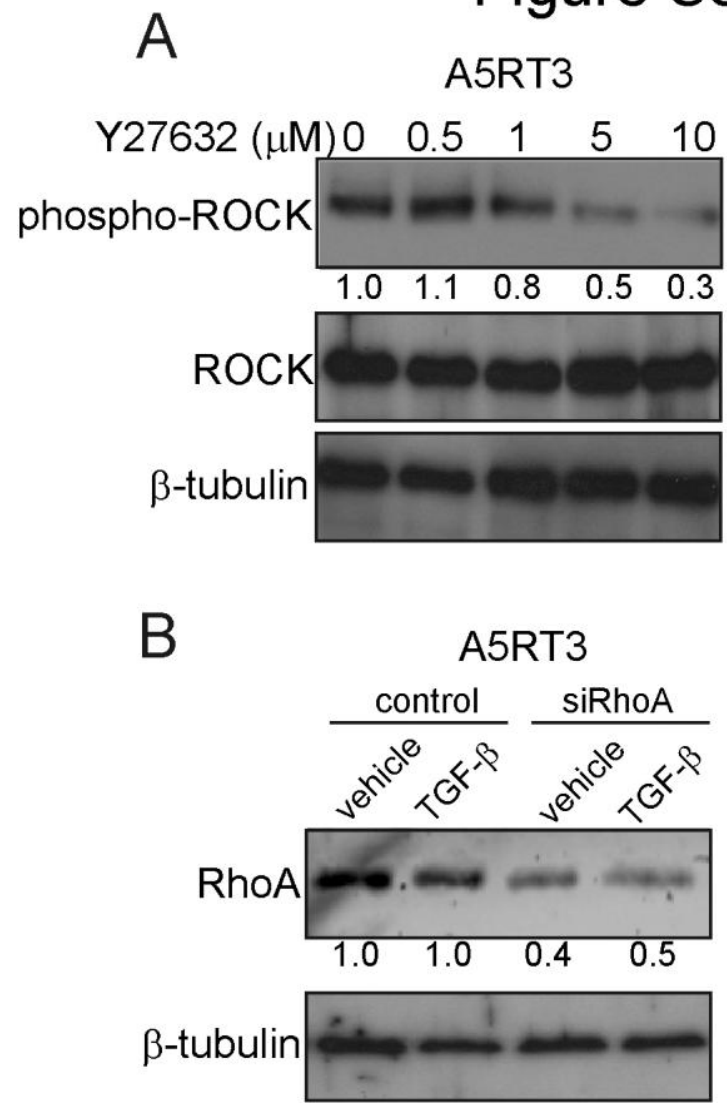




\section{Supplementary Figure Legends}

Figure S1. Comparison of cellular proliferation and death in A5RT3 ${ }_{\text {CTRL }}$ and A5RT3 ${ }_{\text {TAK1 }}$ cells.

(A) The expression levels of TAK1 mRNA and protein in A5RT3 $3_{\text {CTRL }}$ and A5RT3 $3_{\text {TAK1 }}$ cells.

(B-C) Immunoblot analysis of TAK1 downstream signaling mediator, NFאB p65 subunit (B) and ERK and JNK (C) in cells treated with cytokine IL-1 at $20 \mathrm{ng} / \mathrm{ml}$ prior to analysis. $\beta$-tubulin immunoblotting showed equal loading and transfer. Values below each band represents mean fold change compared with control.

(D) FACS analysis of proliferation rate in $\mathrm{A} 5 \mathrm{RT} 3_{\mathrm{CTRL}}$ and $\mathrm{A} 5 \mathrm{RT} 3_{\mathrm{TAK} 1}$ cells as determined by BrdU labelling. Results were gated to show percentage of cell population in the various mitotic phases (ie. G1, S and G2/M). Quadrant percentages are indicated in the respective dot plots.

(E) Phase contrast microscopic images of TAK1-knockdown HSC-5, II4 and MKN28 treated with TGFß1 (10 ng/ml) for $48 \mathrm{~h}$. Scale bar: $100 \mu \mathrm{m}$.

(F) Representative immunoblots of indicated EMT markers of cell samples mentioned in (E). Values below each band represents mean fold change compared with control.

For immuoblotting, $\beta$-tubulin serves as loading control.

Figure S2. TAK1 overexpression in A5RT3 ${ }_{\text {TAK1 }}$ rescued TGFß1-induced EMT.

(A) Phase contrast images of A5RT3 $3_{\mathrm{TAK} 1}$ transfected with expression vector containing TAK1 cDNA and treated with TGF $\beta 1(10 \mathrm{ng} / \mathrm{ml})$ for $48 \mathrm{~h}$. Scale bar: $100 \mu \mathrm{m}$.

(B) qPCR analysis of EMT markers in TAK1-transfected A5RT3 $3_{\mathrm{TAK} 1}$ cells with and without TGF $\beta 1$ treatment. Samples were normalized with reference gene, L27. 
(C) Immunoblot analysis of TAK1-transfected A5RT3 ${ }_{\mathrm{TAK} 1}$ cells for the overexpression of TAK1 and EMT phenotype upon TGF $\beta 1$ treatment. Representative blots were shown with respective densitometry values indicated below lanes, normalised with respect to the control. Samples were normalized with $\beta$-tubulin as a loading control.

Data represent means \pm S.D; $n=3$.

Figure S3. TGFß1-induced EMT is effected through SMAD signaling.

(A) Phase contrast images of SMAD3 siRNA transfected A5RT3 $3_{\text {CTRL }}$ cells with $72 \mathrm{~h}$ of TGF $\beta 1$ (10 ng/ml) treatment. Scale bar: $100 \mu \mathrm{m}$.

(B) qPCR analysis of EMT markers in SMAD3 siRNA transfected A5RT3 ${ }_{\text {CTRL }}$ cells with and without TGF $\beta 1$ treatment. Samples were normalized with reference gene, L27.

(C) Immunoblot analysis of SMAD3 siRNA transfected A5RT3 $3_{\text {CTRL }}$ cells for their knockdown and EMT markers upon TGF $\beta 1$ treatment. Representative blots were shown with respective densitometry values indicated below lanes, normalised with respect to the control. Samples were normalized with $\beta$-tubulin as a loading control.

(D) Immunoblot analysis of phosphorylated SMAD3 in SMAD3-knockdown A5RT3 ${ }_{\text {CTRL }}$ cells. Values below each band represents mean fold change compared with control. For immuoblotting, $\beta$-tubulin serves as loading control.

Data represent means \pm S.D; $n=3$.

\section{Figure S4. Determination of optimal NAC concentration.}

(A-B) Apoptosis assay by annexin V/PI staining (A) and CM-H2DCFDA staining (B) of A5RT3 $3_{\text {TAK1 }}$ cells treated with indicated concentrations of NAC as determined by FACS. Numbers indicate percentages of cells in the respective quadrants. 
(C) Phase contrast microscopy images of A5RT3 ${ }_{\text {CTRL }}$ cells after $48 \mathrm{~h}$ of $100 \mu \mathrm{M}$ NAC treatment.

(D) Representative immunoblots indicated EMT markers in A5RT3 ${ }_{\text {CTRL }}$ cells treated NAC.

Values below each band represents mean fold change compared with control. For immuoblotting, $\beta$-tubulin serves as loading control.

Data represent means \pm S.D; $\mathrm{n}=3$.

\section{Figure S5. Effect of different concentration of Y27632 in ROCK acivity.}

(A) Representative immunoblots of phosphorylated ROCK in A5RT3 ${ }_{\text {CTRL }}$ treated with indicated concentration of ROCK inhibitor Y27632. Values below each band represents mean fold change compared with control. $\beta$-tubulin serves as loading control.

(B) Relative protein expression of RhoA in siRNA RhoA-knockdown A5RT3 cells treated with or without TGF- $\beta 1$.

Data represent means \pm S.D $; n=3$. 
Supplemental Table 1- qPCR Primer sequences used in this study.

\begin{tabular}{|l|l|}
\hline TAK1 qPCR primer 1 & GTTCCGTGTAAGGGCTTTGA \\
\hline TAK1 qPCR primer 2 & GAGCAGCTGCCACTTACCTTTACA \\
\hline E-cadherin qPCR primer 1 & GCCGAGAGCTACACGTTCA \\
\hline E-cadherin qPCR primer 2 & GACCGGTGCAATCTTCAAA \\
\hline N-cadherin qPCR primer 1 & TGCGGTACAGTGTAACTGGG \\
\hline N-cadherin qPCR primer 2 & GAAACCGGGCTATCTGCTCG \\
\hline Vimentin qPCR primer 1 & GTTTCCCCTAAACCGCTAGG \\
\hline Vimentin qPCR primer 2 & AGCGAGAGTGGCAGAGGA \\
\hline SNAI1 qPCR primer 1 & TCGGAAGCCTAACTACAGCGA \\
\hline SNAI1 qPCR primer 2 & AGATGAGCATTGGCAGCGAG \\
\hline L27 qPCR primer 1 & TGATGGCACCTCAGATCGC \\
\hline L27 qPCR primer 2 & AGAGTACCTTGTGGGCATTAGG \\
\hline
\end{tabular}




\section{Reprogramming of Cellular Bioenergetics Primes Cancer Cells for Epithelial-Mesenchymal Transition}

Ziqiang TEO, Ming Keat SNG, Jeremy Soon Kiat CHAN, Yinliang LI, Liang LI, Maegan Miang Kee LIM, Terri PHUA, Pengcheng ZHU, Nguan Soon TAN*

*Corresponding Author.

Submitted Manuscript. 
Reprogramming of cellular Bioenergetics Primes cancer cells for EpithelialMesenchymal Transition

Ziqiang $\mathrm{TEO}^{1}$, Ming Keat $\mathrm{SNG}^{1}$, Jeremy Soon Kiat $\mathrm{CHAN}^{1}$, Yinliang $\mathrm{LI}^{1}$, Liang $\mathrm{LI}^{1}$, Maegan Miang Kee LIM ${ }^{1}$, Terri PHUA ${ }^{1,2}$, Pengcheng ZHU ${ }^{1}$, Nguan Soon TAN ${ }^{1,3,4}$

${ }^{1}$ School of Biological Sciences, Nanyang Technological University, 60, Nanyang Drive, Singapore 637551.

${ }^{2}$ Karolinska Institutet, Department of Microbiology, Tumor and Cell Biology. Nobels väg 16, Stockholm 17177, Sweden

${ }^{3}$ Institute of Molecular and Cell Biology, 61 Biopolis Drive, Proteos, Singapore 138673.

${ }^{4}$ KK Research Centre, KK Women's and Children's Hospital, 100 Bukit Timah Road, Singapore 229899

Correspondence:

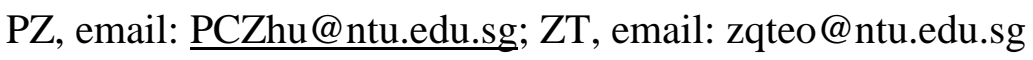

NST (senior corresponding author), email: nstan@ntu.edu.sg, Tel: +65-6316-2941, Fax: +6567913856 


\section{Summary}

Background: Epithelial-mesenchymal transition (EMT) empowering cancer cells with enhanced invasive capabilities. Although molecular and cellular changes are well-studied during EMT, little is known about bioenergetics changes during EMT and the specific molecular players that reprogram cancer cell bioenergetics to drive EMT.

Methods: Three in vitro EMT model, a novel dual-inducible EMT mouse model and clinical patient's samples were used to characterize bioenergetics changes during EMT. Cellular levels of ATP, ADP and AMP were measured using reverse-phase HPLC and used to determine cancer cells' energy charge status after indicated treatment. Comparative microarray analysis and gene knock-down assays were used to identify critical players that reprogrammed cancer cell metabolism during EMT. The mechanism was elucidated using a combination of proximity ligation assay, immunofluorescence, immunoblots and protein and chromatin immunoprecipitation.

Results: We found that EMT is an energy-demanding process fueled by glucose metabolismderived ATP. We identified angiopoietin-like 4 (ANGPTL4) as an important driver of cancer cell metabolism within a larger EMT gene program. ANGPTL4-knockdown suppresses energy charge elevation, delaying EMT and reduces cancer metastasis to the lung and liver. Unbiased kinase inhibitor screens revealed that ANGPTL4 differentially regulates the expression of 14-3-3 adaptor proteins to synchronize metabolic shift necessary for EMT.

Conclusions: Our study shows that metabolic reprogramming is necessary to prime cancer cells for EMT competency. We identified a novel ANGPTL4:14-3-3 signaling axis which coordinates energy demand and stability of critical EMT proteins necessary to drive EMT and promote metastasis. These findings offer new therapeutic opportunities for metastasis with important clinical implications. 


\section{Introduction}

Metastasis, the spread of cancer cells to distant tissues and/or organs, is a complex and multi-step process, making it a challenging therapeutic target (1). Metastasis accounts for more than $90 \%$ of cancer mortality (2). Initiation of the metastatic process resembles the epithelial-mesenchymal transition (EMT) that occurs during embryogenesis and wound healing (3-5). During EMT in cancer, epithelial cells lose their cell polarity and cell-cell adhesions are weakened as they adopt a mesenchymal phenotype. This change is accompanied by cytoskeletal remodeling, ultimately allowing the cells to gain motility and the ability to invade distant organs (5). These energy-demanding processes during cancer EMT suggest that changes in cellular metabolism may be required to drive the EMT event.

Metabolic reprogramming has been recognized as one of the 10 hallmarks of cancer (6). Our current understanding of cancer metabolism is based primarily on metabolic status comparison between primary tumors and normal healthy cells. Cancer cells derive a substantial amount of ATP from aerobic glycolysis (i.e., the Warburg effect) instead of oxidative phosphorylation to support their anabolic growth and proliferation (7). Cancer cells also exploit glutaminolytic flux, amino acid and lipid metabolism, mitochondrial biogenesis, the pentose phosphate pathway, and macromolecular biosynthesis to progress malignancy $(8,9)$. Recent studies have suggested that oncogenes and tumor suppressors function as critical modulators of metabolic reprogramming to support tumor progression (10,11). Despite the significance of EMT in metastasis, little is known about the changes in cellular bioenergetics that occur during this process and whether such metabolic reprogramming is a functional prerequisite.

The exponential growth of the primary tumor exposes cancer cells to various microenvironmental stresses, including hypoxic, acidic, and inflammatory milieus $(3,4)$. Interestingly, though these constraints can kill cancer cells, they also exert selective pressures 
on cells to exploit adverse microenvironments by modifying their cellular behavior and selecting for a highly invasive phenotype that facilitates metastasis (12). Microenviromental stimuli initiate the EMT process by controlling the expression and function of a myriad of molecular players, such as Snai1, ZEB-1, Wnt ligands and matricellular proteins which are pivotal for EMT $(3,13,14)$. However, majority of these players are unable to initiate EMT alone, suggesting that there are unique molecular drivers capable of driving the EMT event (15-17). Interestingly, hypoxia and TGF- $\beta$, which are known initiators of EMT, can also modulate cancer cell metabolism during tumorigenesis $(12,18)$. Hence, whether these microenvironmental signals can influence specific molecular players to reprogram cancer cell bioenergetics and drive EMT remains unknown.

Here, we show how EMT is coordinated with cancer cell metabolism. Using in vitro, in vivo EMT models, stage-specific human tumor biospies, comparative microarray analysis, and energy charge measurements, we showed that reprogramming of cancer cells bioenergetics is required for EMT. We further identified angiopoietin-like 4 (ANGPTL4) as a key player in cellular bioenergetics and elucidated the mechanism by which it coordinates the energy demands required for EMT. 


\section{Results}

\section{Altered cancer cell bioenergetics during EMT}

To examine whether EMT utilizes cellular energy, we carried out a series of molecular and biochemical analyses to examine changes in cellular bioenergetics during EMT. First, we established three in vitro EMT models using the polarized gastric carcinoma cell line MKN74. In two models, EMT was initiated by either hypoxia $\left(1 \% \mathrm{O}_{2}\right.$, Figure 1A) or TGF- $\beta$ (Supplementary Figure 1A, available online) to mimic the microenvironment of stress-induced EMT. The third model utilized the transgenic MKN74 cell line harboring a Snai1-ER transgene (MKN74 $\left.{ }_{\text {SnailER }}\right)$ that allows direct initiation of EMT by 4hydroxytamoxifen (4-OHT) (Figure 1B; Supplementary Figure 1B, available online). Upon exposure to either microenvironmental stress or 4-OHT, the epithelial colonies of MKN74 cells scattered and adopted a mesenchymal-like phenotype, with diminished E-cadherin staining bordering the epithelial cells, after $48 \mathrm{~h}$ (Figure 1A; Supplementary Figure 1A, available online) and $96 \mathrm{~h}$ (Figure 1B), respectively. qPCR and immunoblotting for critical EMT genes showed downregulation of epithelial genes (CDH1; DDR1; ErBb3), with a concomitant increase in mesenchymal gene expression (Snai1; ZEB-1; Figure 1, A and B; Supplementary Figure 1A, available online). Similar EMT phenotype was also observed in MCF7, II4, HSC, and HepG2 cancer cell lines (Supplementary Figure 4D-G, available online).

Cancer cells are known to exploit aerobic glycolysis to metabolize glucose to lactate instead of pyruvate, resulting in the acidification of the extracellular environment (7). We observed increased glucose uptake and extracellular acidification rate in MKN74 $4_{\text {CTRL }}$ and MKN74 $_{\text {SnailER }}$ cells during EMT, suggesting that glycolysis was augmented (Figure 1, C and D). In vivo 2-deoxyglucose (2-DG) imaging of our inducible EMT xenograft mice, transplanted with MKN74 $_{\text {SnailER }}$ cells, showed similar elevation in glucose uptake in 4-OHT- 
treated tumors compared to vehicle-treated tumors (Figure 1E). To further confirm that cellular metabolism was altered during cancer EMT, we investigated the energy charge status of the cells which is indicative of the cellular metabolic activity (19-21). We observed an increased energy charge in MKN74 $4_{\text {CTRL }}$ and MKN74 $4_{\text {SnailER }}$ cells during EMT progression, which mirrored the increased glycolytic activity (Figure 1F). This increase in energy charge during EMT was also observed in four different cancer cell lines and our in vivo xenograft tumors (Figure 1, G and H). Importantly, clinical samples showed significant correlations between the energy charge status and tumor grades, with the higher grade metastatic tumors exhibiting a higher energy charge (Figure 1I; Supplementary Table 3). These observations suggest that cancer cells undergo metabolic reprogramming during EMT and the increased cellular bioenergetics could fuel the aggression of the metastatic cancer cells.

\section{Metabolic reprogramming enhanced metastatic capability of cancer cells}

Cancer EMT is often accompanied by energy-intensive processes like cytoskeletal remodeling and enhanced drug resistance, ultimately transforming these cancer cells into malignant forms with superior survivability and invasiveness (22-25). The observations above prompted us to examine whether alteration of cancer cellular bioenergetics can influence malignant behavior. The PI3K/AKT and AMPK pathways are two major regulatory nodes for cellular bioenergetics (26). Therefore, dominant-negative and constitutive forms of AKT and AMPK were introduced into MKN74 ${ }_{\text {CTRL }}$ and MKN74 $4_{\text {SnailER }}$ cells (Supplementary Figure 2A, available online). Constitutive active AKT (caAKT) and AMPK (caAMPK) increased energy charge status of both MKN74 $4_{\text {CRL }}$ and MKN74 $4_{\text {SnailER }}$ cells (Figure 2A-D). Conversely, dominant-negative AKT (dnAKT) and AMPK (dnAMPK) resulted in a decrease in the energy charge status of these cells (Figure 2A-D). In the absence of EMT-inducing signals, the colony morphology of MKN74 cells harboring caAKT (MKN74 $\left.4_{\text {caAKT }}\right)$, dnAKT $\left(\right.$ MKN74 $\left._{\mathrm{dnAKT}}\right)$, caAMPK $\left(\right.$ MKN74 $\left._{\text {caAMPK }}\right)$ and dnAMPK $\left(\right.$ MKN74 $\left._{\mathrm{dnAMPK}}\right)$ resembled that of 
MKN74 $_{\text {CTRL }}$ cells. Interestingly, upon exposure to hypoxia or 4-OHT, MKN74 $4_{\text {caAKT }}$ and MKN74 caAMPK cells exhibited a more pronounced EMT phenotype, as early as $24 \mathrm{~h}$ after hypoxia exposure, compared to $48 \mathrm{~h}$ in cognate MKN74 ${ }_{\mathrm{CTRL}}$ cells (Figure 2, E and F). The dominant-negative forms of AKT and AMPK suppressed energy charge elevation, delaying EMT progression (Figure 2A-F; Supplementary Figure 2B, available online). These observations suggest that the consolidation of cellular bioenergetics may be a pre-requisite to prime the cancer cells for EMT.

To gain further insights into the biological significance of altered cancer cellular bioenergetics during EMT, we created an inducible EMT xenograft mouse model using MKN74 $_{\text {SnailER }}$ cells. Histological analysis of tumor sections revealed a compact vehicletreated control tumors with clear encapsulation of the tumor mass, defined by strong distinct E-cadherin and basement membrane laminin 332 staining within the tumors (Figure 2G; Supplementary Figure 2C, available online). Conversely, 4-OHT-treated tumors appeared dispersed with diminished E-cadherin staining at the cell-cell borders and clear disruption of basement membrane laminin 332 compared to vehicle-treated tumors, indicative of an aggressive tumor phenotype (Figure 2G; Supplementary Figure 2C, available online). Indeed, elevated activities of pro-invasive matrix metalloproteinases (MMPs)-2 and -9 were detected in 4-OHT-treated tumors and these mice harbored significantly more lung metastases than the vehicle control mice (Figure 2, H and I). Likewise, we also observed improved xenobiotic resistance of cancer cells during EMT. One of the most important resistance mechanisms is the active efflux of anti-cancer drugs via the ATP-binding cassette (ABC) transporter family. Drug efflux was enhanced during EMT and the exposure of MKN74 $4_{\text {TRL }}$ and MKN74 $4_{\text {SnailER }}$ cells to chemotherapeutic drug, cisplatin, during EMT resulted in a significant reduction in cellular apoptosis (Figure 2, $\mathrm{J}$ and $\mathrm{K}$ ). The $\mathrm{ABC}$ transporters expression was also elevated during EMT as revealed by focus array analysis (Supplementary Figure 2D, available online). 
This was further confirmed by immunoblot analysis of multidrug resistant receptor 1 (MDR1), a major ABC transporter in tumor (Figure 2, L and M).

Altogether, our findings indicated that the increased cellular bioenergetics during EMT could fuel malignant transformation, conferring enhanced invasive and chemoresistant capabilities to the cancer cells.

\section{ANGPTL4 regulates cellular bioenergetics during EMT}

EMT induction signals could influence various molecular players to modify cancer cells behaviors and drive EMT. To identify driver genes involved in metabolic reprogramming during EMT from differentially expressed genes associated with cellular metabolism, we performed knockdown experiments followed by microarray analysis. We observed an unanimous enrichment of metabolic regulatory genes, particularly in glucose, fatty acid, autophagy, amino acid, and ROS metabolism, as early as $24 \mathrm{~h}$ after EMT across the three in vitro EMT models (Figure 3, A and B). qPCR and immunoblot analysis of selected metabolic genes and ROS production measurements further confirmed that these processes were enhanced during EMT, which could contribute to the elevated energy charge of the cancer cells during EMT (Figure 3, C and D; Supplementary Figure 3B, available online). Interestingly, we found elevated angiopoietin-like 4 (ANGPTL4) expression in several gene clusters across all EMT models. ANGPTL4 protein levels, specifically the Cterminal fibrinogen-like form (cANGPTL4), were elevated during EMT in both in vitro models and in vivo xengraft tumors (Figure 3, E and F; Supplementary Figure 3C, available online). Furthermore, clinical analysis revealed that metastatic cancers expressed higher ANGPTL4 expression, which suggested important roles for ANGPTL4 in regulating cellular bioenergetics during EMT (Figure 3G).

ANGPTL4 has well-defined roles in peripheral metabolism, however, its roles in cellular metabolism remains unknown. The suppression of ANGPTL4 resulted in significant 
alteration of $>50 \%$ of genes identified in our microarray analysis (Figure 3B). Treatment with either neutralizing antibodies ( $\alpha$-cANGPTL4) or siRNA against ANGPTL4 (siANGPTL4) diminished glucose uptake and energy charge elevation in MKN74 $4_{\mathrm{CTRL}}, \mathrm{MKN} 74_{\text {SnailER }}$ and 4 other cancer lines during EMT. Conversely, the addition of exogenous rh-cANGPTL4 alone was able to rescue glucose uptake and energy charge status (Figure 3H-K; Supplementary Figure 3C-F, available online). To further understand the role(s) of ANGPTL4 in controlling cancer cells' bioenergetics, we created a dual-inducible EMT xenograft mouse model. A doxycycline (Dox)-inducible shANGPTL4 expression plasmid was introduced into MKN74 $_{\text {SnailER }}$ cells to generate the dual-inducible MKN74 $4_{\text {SnailER:shANGPTL4 }}$ cell line prior to xenograft (Supplementary Figure 3G, available online). In vivo xenograft tumors exhibited a similar elevation in glucose uptake and energy charge status ( 1.5-fold) during EMT progression, and this elevation was diminished by Dox-diet-induced ANGPTL4 depletion (Figure 3L; Supplementary Figure 3H, available online). These changes in the cellular energy charge corresponded with the intracellular ATP levels, lending further credence to the importance of ANGPTL4 in regulating cellular bioenergetics during EMT (Supplementary Figure 3I-K, available online).

\section{Metabolic reprogramming by ANGPTL4 augments EMT}

Our observations above prompted us to investigate whether metabolic reprogramming by ANGPTL4 could influence the malignant transformation of cancer cells during EMT. The depletion of ANGPTL4 delayed EMT, with the cells retaining their epithelial-like morphology characterized by clear E-cadherin staining and reduced nuclear accumulation of Snai2 (Figure 4, A and B; Supplementary Figure 4, A and H available online). qPCR and immunoblot analysis revealed a delay in the expression of critical EMT genes (Figure 4, A and B; Supplementary Figure 4B, D-G, available online). Interestingly, MKN74 cells treated with recombinant human cANGPTL4 (rh-cANGPTL4) resulted in the repression of epithelial 
markers and significant elevation of mesenchymal markers (Figure 4C; Supplementary Figure 4C, available online).

The depletion of ANGPTL4 in our dual-inducible EMT xenograft model rescued Ecadherin and laminin 332 expression in the tumors compared to the 4-OHT-treated tumors (Supplementary Figure 4I, available online). Pro-invasive MMPs activities and secondary metastases in the lungs were also significantly decreased (Figure 4, D and E). Furthermore, immunofluorescence analysis of benign and metastatic mouse skin tumor sections revealed that metastatic tumors expressed high levels of ANGPTL4 that is correlated to diminished Ecadherin expression (Figure 4F). Finally, the depletion of ANGPTL4 was able to sensitize MKN74 $_{\text {CTRL }}$ and MKN74 $4_{\text {SnailER }}$ cells to xenobiotics and suppressed MDR1 expression during EMT (Figure 4G-I). These observations suggest that ANGPTL4 can augment malignant transformation by controlling cellular bioenergetics to drive EMT.

\section{ANGPLT4 regulates specific 14-3-3 expression}

The 14-3-3 protein family consists of adaptor proteins that play critical roles in numerous important cellular functions via binding to specific phosphorylation signals on target proteins (27). During wound healing, keratinocytes adopt a migratory mesenchymal phenotype highly reminiscent of cancer EMT (28). We demonstrated that ANGPTL4 can influence the expression of specific members of the 14-3-3 protein family during wound healing. Thus, we examined the expression of all seven 14-3-3 protein isotypes during cancer EMT. In response to EMT-inducing signals, MKN74 $4_{\text {CTRL }}$ and MKN74 $4_{\text {SnaileR }}$ cells exhibited

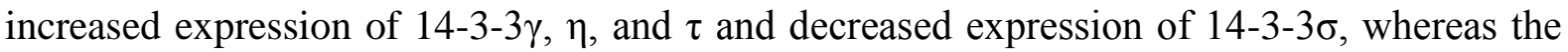
remaining 14-3-3 isotypes were unchanged (Figure 5, A and B). Suppression of ANGPTL4 resulted in a significant decrease in the expression of $14-3-3 \gamma$ compared to $14-3-3 \eta$ and $\tau$, whereas the exposure of MKN74 ${ }_{\text {CTRL }}$ cells to rh-cANGPTL4 enhanced 14-3-3 $\gamma$ expression (Figure 5, A and C). Notably, tumor biopsies from patients exhibited similar alterations in 14- 
3-3 $\gamma$ expression as in four other cancer cell lines during EMT (Figures 5I; Supplementary Figure 5B-E, available online). These observations suggest that ANGPTL4 may exert its influence through these 14-3-3 isotypes to reprogram cancer cell bioenergetics and drive EMT.

Although a multitude of studies demonstrated the importance of 14-3-3 in various signaling pathway, how specific 14-3-3 expression are regulated remains unclear. Exposure of MKN74 ${ }_{\text {CTRL }}$ cells to rh-cANGPTL4 increased the expression of 14-3-3 $\gamma, \eta$, and $\tau$ mRNA within 6 hours, while 14-3-3 $\sigma$ expression was suppressed (Supplementary Figure 5A, available online). To obtain insight into the signaling pathways involved in ANGPTL4mediated expression of 14-3-3 isotypes, we performed an unbiased kinase inhibitor screen to identify key signaling mediators whose inhibition would suppress ANGPTL4-mediated upregulation of 14-3-3 expression. qPCR followed by Ingenuity Pathway Analysis showed that the majority of involved kinases converged on the PI3K/AKT and MAPK signaling cascades, implicating several transcription factors known to be involved in cancer progression (Figure 5D). We further showed that MKN74 cells exposed to hypoxia, 4-OHT and exogenous rhcANGPTL4 displayed increased phospho-activation of AKT, whereas the depletion of ANGPTL4 opposed this effect during hypoxia-induced EMT (Figure 5E-G). Analysis of four different cancer cell lines, xenograft EMT tumors and tumor biopsies from patients revealed similar phospho-activation of AKT dependent on ANGPTL4 (Figure 5I; Supplementary Figure 5B-E, available online). Importantly, immunoblot analysis of the identified transcription factors showed that three of these transcription factors, namely CREB, cFOS, and Stat3, were phospho-activated during EMT (Figure 5J). These observations confirm that ANGPTL4 can activate specific transcription factors via the PI3K/AKT pathway to differentially regulate specific 14-3-3 isotypes.

\section{The ANGPTL4:14-3-3 $\gamma$ axis coordinates energy demand during EMT}


To further decipher how ANGPTL4:14-3-3 $\gamma$ axis control cellular metabolism during EMT, we interrogated the ANnotation and Integrated Analysis of the 14-3-3 interactome (ANIA) database to find key signaling mediators of cancer cell metabolism and pivotal transcription factors in EMT that could interact with 14-3-3 (29). Remarkably, our analysis identified tuberin sclerosis complex (TSC2) and Snai1 as highly ranked interaction partners for 14-3-3. TSC2 is an important gatekeeper of cellular metabolism that integrates extracellular signals to direct anabolic or catabolic activities (30,31). Depending on the TSC2 phosphorylation sites, 14-3-3 can bind and inhibits TSC2 activities. In our experiment, inhibitory TSC2 phosphorylation at Ser939 (TSC2 ${ }_{\text {S939) }}$ was increased during EMT (Supplementary Figure 6A-C, available online). Co-immunoprecipitation and proximity

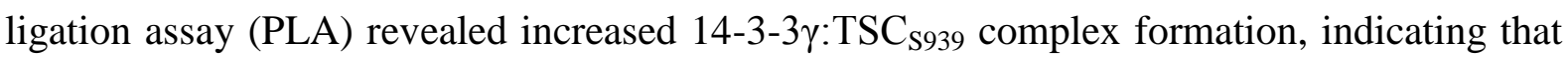
TSC2 was suppressed during EMT (Figure 6A-D; Supplementary Figure 6E-F and 7A-C, available online). Conversely, ANGPTL4 deficiency diminished 14-3-3 $\gamma: \mathrm{TSC}_{\text {S939 }}$ complex formation (Figure 6A-D; Supplementary Figure 6E-F, available online). These observations revealed a novel ANGPTL4:14-3-3 signaling axis that exerts significant influences to control cellular bioenergetics during EMT.

In vitro co-immunoprecipitation analysis and PLA of Snai1 and 14-3-3 revealed a significant increase in 14-3-3 $\gamma$ :Snail complex formation during hypoxia-induced EMT. This interaction was diminished in the absence of ANGPTL4, while Snai1:14-3-3 $\eta / \tau$ complex formation remained unchanged during EMT (Figure 6E-H; Supplementary Figure 6G-H, available online). Similarly, PLA analysis revealed significantly more 14-3-3 $\gamma$ :Snai1 complexes in 4-OHT-treated tumors than in vehicle-treated tumors from mice fed a normal diet (Figure 6G). Tumor sections from 4-OHT-treated mice fed a Dox diet exhibited fewer 14-3-3 $\gamma:$ Snai1 interactions than mice fed a normal diet (Figure 6G). PLA analysis of tumor biopsies from patients further confirmed that higher grade, more aggressive tumors harbored 
significantly more 14-3-3 $\gamma$ :Snail complexes than their benign counterparts (Figure $6 \mathrm{H}$; Supplementary Figure 7D-F, available online). The interaction of 14-3-3 with Snai1 was proposed to stabilize Snai1 binding on the E-cadherin promoter to facilitate transcriptional repression during EMT (32). We showed that the reduction in 14-3-3 $\gamma$ :Snai1 complex in MKN74 $_{\triangle \mathrm{ANGPTL} 4}$ cells resulted in decreased Snail occupancy of the E-cadherin promoter during hypoxia-induced EMT, indicating that the repression of E-cadherin gene expression was impaired. Furthermore, EMT was delayed in the absence of ANGPTL4, highlighting the importance of 14-3-3 $\gamma$ :Snail complexes during malignancy (Figure 6I).

Taken together, our data revealed a previously unknown mechanism where the ANGPTL4:14-3-3 $\gamma$ signaling axis coordinates energy demand and stability of critical EMT proteins necessary to drive EMT (Figure 6J). 


\section{Discussion}

The EMT is the first step in metastasis initiation and confers invasive characteristics to cancer cells (4). The chronic exposure of cancer cells to microenvironmental stresses, such as hypoxia and inflammation, allows the selection of EMT-competent cells in cancer (33). Incidentally, these stresses also modify cancer cell metabolism to sustain their growth potential (26). However, whether such metabolic reprogramming is a prerequisite for EMT remains unclear. Using a combination of in vitro, in vivo and clinical models, we found that reprogramming and consolidation of cellular bioenergetics are required to prime cancer cells for EMT. Furthermore, the elevated cellular bioenergetics can fuel malignant transformation and enhanced cancer metastasis. Conceivably, there may be molecular drivers that modulate metabolic reprogramming and prime these cancer cells for EMT competency. Microarray analysis of three EMT models revealed ANGPTL4 as an important molecular driver of metabolic changes during EMT. We went on to demonstrate that ANGPTL4 exert its influence through a novel ANGPTL4:14-3-3 signaling axis to coordinate energy demand and stability of critical EMT proteins necessary to drive EMT.

Metastasis requires the cooperative effects of multiple signaling events and biological progresses $(3,33)$. While the role of cellular metabolism is well-known during cancer cell proliferation, its role during malignant progression remains unclear. During metastasis initiation, the primary cancer cells undergo major alterations in cell morphology and functions to gain a pro-invasive and chemoresistance phenotype. We demonstrated that during EMT, the cancer cells increase glucose uptake and aerobic glycolysis to reprogram their cellular bioenergetics, which may be necessary to fuel the acquisition of these malignant traits. The active remodelling of the actin cytoskeleton requires ATP binding and hydrolysis to confer invasive abilities to the cancer cells $(22,23)$. Similarly, the enhanced chemoresistance involved active efflux of various chemotherapeutic compounds from cancer cells (24). Thus, it 
is conceivable that metabolic reprogramming can prime the cancer cells for EMT. Interestingly, when we enhanced the cancer cellular biogenergetics by constitutively activating PI3K/AKT and AMPK pathways, these cancer cells showed accelerated transition into mesenchymal forms. Importantly, the suppression of cellular bioenergetics hindered EMT progression, suggesting that the alteration of cellular bioenergetics during EMT primes these cancer cells for increased mobility and xenobiotic-resistance. Moreover, the loss of Ecadherin has been shown to augment glycolysis to favor EMT. Thus, our study revealed that the consolidation of cellular bioenergetics is essential for metastasis initiation.

Cancer cells are known to express higher ANGPTL4 levels compared to their normal counterparts. As the tumor progresses, the chronic exposure of the cancer cells to microenvironmental signals can triggers further elevation in ANGPTL4 expression. This suggest that ANGPTL4 could play significant roles during malignant progression (34-37). Many studies have linked ANGPTL4 to cancer metastasis, from enhancement of metastatic cells motility, angiogenesis to the disruption of vascular integrity to facilitate distal dissemination. Although ANGPTL4 has well-established roles in peripheral metabolism, its role in cellular metabolism is unknown. By demonstrating ANGPTL4 as a molecular driver of metabolic changes during EMT, we provide a novel insight for its function in pathological metabolism. By suppressing ANGPTL4, we are able to reconfigure cancer cell metabolism and delay EMT. In vitro and in vivo EMT models showed that cancer cells deficient in ANGPTL4 were unable to rewire aerobic glycolysis to increase cellular energy charge during EMT, resulting in reduced metastasis. These suggest that ANGPTL4 can consolidate the cellular bioenergetics of cancer cells and prime them to an "excited" state for EMT. Notably, all of these changes were also detected in clinical tumor biopsies and strongly correlated with the stage and aggressiveness of the tumors, further underscoring the clinical relevance of our 
findings. It will be interesting to explore if cancer cells within tumors have energetic "threshold" for EMT competency.

A key mechanistic insight is that ANGPTL4 regulates the expression of specific isoforms of 14-3-3 and contributes to an important new signaling axis that coordinates multiple biological processes for metastasis. Interestingly, the functions of many mediators in autophagy, glycolysis, survival, and cell cycle, among others, are known to be regulated by the 14-3-3 protein family $(27,38)$. The expression of $14-3-3$ protein was also aberrantly upregulated in numerous tumor types (39-41). However, due to the diverse influences 14-3-3 proteins has over critical cellular pathways, the development of inhibitors targeting 14-3-3 remains a challenge (39-41). In contrast, ANGPTL4 is a secreted extracellular protein that can be easily targeted by either small molecules or antibodies. Furthermore, the elevated expression of ANGPTL4 and its multi-faceted roles in cancer makes it an attractive therapeutic target. Therefore, through the inhibition of ANGPTL4, we may be able to apply a multi-prong approach to inhibit tumor progression and EMT, keeping the tumors in a lowgrade status, potentially increasing therapeutic efficacy of cancer treatments.

Our study shows that metabolic reprogramming in cancer cells is a pivotal event in the progression of EMT. Our findings identified the involvement of the ANGPTL4:14-3-3 signaling axis that coordinates multiple biological processes for metastasis and revealed a previously unknown role for ANGPTL4 as a molecular driver of cellular metabolism in the EMT to promote metastasis with important clinical implications. 


\section{Experimental Procedures}

Antibodies. Antibodies for E-cadherin, pan-AKT, p-AKT (S473), AMPK, p-AMPK (T172), TSC2, p-TSC2, CREB, p-CREB, cFOS, p-cFOS, ELK1, p-ELK1, STAT3, p-STAT3 and various 14-3-3 isotypes (Cell Signaling, USA); N-cadherin and Snai2 (Millipore, USA); $\beta$ -

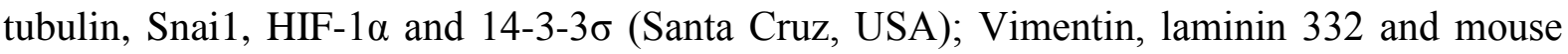
monoclonal anti-human cANGPTL4 mAb11F6C4 (Abcam, USA); pan-cytokeratin and IRdye 680-conjugated secondary antibodies (Thermo Scientific, USA); Alexa Fluor 488conjugated secondary antibodies and Alexa Fluor 594-conjugated phalloidin (Molecular Probes, USA). Rabbit polyclonal anti-human cANGPTL4 were produced in-house.

Cell culture. MKN74 human gastric carcinoma (ATCC, USA) were cultured in RPMI-1640 supplemented with $10 \% \mathrm{FBS}$ in a humidified atmosphere of $5 \% \mathrm{CO}_{2}$ at $37{ }^{\circ} \mathrm{C}$. All other cell lines were cultured in DMEM with 10\% FBS. All ANGPTL4 knock-down cells were generated as per manufacturer's protocol (Thermo Scientific, USA) with DharmaFECT transfection reagent and Smartpool siRNA against ANGPTL4. AKT activity was measured using the AKT activity kit (Cell Signaling, USA).

Human tumor biospies. Fresh frozen breast, colon, gastric and head and neck tumor biospies (Proteogenex, USA) were used for protein, energy charge and immunofluorescence analysis.

Retrovirus transduction. MKN74 $4_{\text {SnailER }}$ cells were generated using retroviral transduction as previously described (42). Briefly, HEK 293 T cells were transfected with pCL-10A1 retrovirus packaging vector (Imgenex, USA) and pWZL-Snai1-ER (Addgene, USA). 
Harvested pseudoviruses were used to infect MKN74 cells. Following transduction, cells were selected with $5 \mu \mathrm{g} / \mathrm{mL}$ of blasticidin hydrochloride (Sigma Aldrich, USA).

Generation of dual-inducible MKN74 $_{\text {Snai1ER/shANGPTL4 }}$ cell line. Doxycycline-inducible pSingle-tTS-shRNA vector carrying either shANGPTL4 or scrambled shRNA was introduced into MKN74 $_{\text {SnailER }}$ cells using Fugene HD reagent as per manufacturer protocol (Promega, USA). MKN74 $4_{\text {SnailER/shANGPTL4 }}$ and MKN74 $4_{\text {Snai1ER/shscrambled }}$ cells were selected with $1 \mathrm{mg} / \mathrm{mL}$ of G418 (Sigma Aldrich, USA). The efficiency of knockdown was verified by qPCR and immunoblotting after treatment with various concentrations of doxycycline (up to $10 \mu \mathrm{g} / \mathrm{mL}$ ).

In vitro induction of EMT. Cells were seeded at a density of $5.25 \times 10^{2} \mathrm{cell} / \mathrm{cm}^{2}$ to allow the formation of individual colonies before indicated treatments. Hypoxia treatments were performed in hypoxic chamber (Stem cell Technology, USA) purged with 5\% $\mathrm{CO}_{2}$ and $95 \%$ $\mathrm{N}_{2}$ to obtain $\mathrm{O}_{2}$ concentration at $1 \%$ as determined by an $\mathrm{O}_{2}$ sensor. For TGF- $\beta$-induced EMT, cells were treated with $10 \mathrm{ng} / \mathrm{mL}$ of TGF- $\beta$ for 2 days. For Snail-mediated EMT, MKN74 $_{\text {SnailER/shANGPTL4 }}$ and MKN74 $_{\text {SnaileR }}$ cells were exposed to $20 \mathrm{ng} / \mathrm{mL}$ of 4hydroxytamoxifen (4-OHT) for 4 days.

In vivo induction of EMT. Six-week-old BALB/c athymic female nude mice (20-22 g) were injected subcutaneously with either $1 \times 10^{6}$ MKN74 $4_{\text {SnailER/shANGPTL4 }}$ or MKN74 $4_{\text {SnailER/shscrambled }}$ cells resuspended in matrigel. A week after xenograft was established, each xenograft group was randomly divided into four subgroups. Two subgroups received intraperitoneal injection of 4-OHT (4 mg/kg) and were fed on either normal chow diet or Dox-diet (625 mg/kg, Harlan Laboratories, USA). The other two subgroups received vehicle and similarly fed on the two different diets. Injection was done twice weekly using sunflower seed oil as carrier for 4- 
OHT. After 8 weeks, the mice were sacrificed. The tumors and lungs were harvested for further analysis. Animal experiments were carried out in accordance to the guidelines of the University Institutional Animal Care and Use Committee (IACUC, ARF-SBS/NIEA0250AZ), Singapore.

Chromatin-immunoprecipitation (ChIP) and Re-ChIP. ChIP experiments were carried out as previously described with slight modifications (43). Briefly, chromatin was cross-linked using $0.5 \%$ formaldehyde for $10 \mathrm{~min}$ at $37^{\circ} \mathrm{C}$ and sonicated in SDS lysis buffer (1\% SDS, 10mM EDTA, and 50mM Tris-HCl, pH 8.1) to obtain DNA fragments of 200-500 bp. $10 \%$ of the supernatant was retained as input, while the remaining amount was immunoprecipitated using anti-Snai1 antibody (Santa Cruz, USA) and immunocomplexes were affinity precipitated by Protein A/G Sepharose (Santa Cruz, USA). Re-ChIP was performed by subsequent probing with anti-14-3-3 $\gamma$ antibody (Thermo Scientific, USA). DNA fragments were reverse cross-linked at $65{ }^{\circ} \mathrm{C}$ for $6 \mathrm{~h}$. The ChIP primer sequences are listed in Supplementary Table S1.

Energy charge determination. The cellular levels of AMP, ADP and ATP were determined by HPLC. Nucleotides were extracted as previously described (44). Nucleotides standards (Sigma Aldrich, USA) were prepared in milli-Q water and resolved on a Shimadzu LC-20AD series Quaternary Gradient HPLC system with PDA detector using a Hypersil ODS 150 mm x $4.6 \mathrm{~mm}$ I.D. C18 reverse-phase column with $3 \mu \mathrm{m}$ particle size and $5 \mu \mathrm{m}$ Hypersil ODS C18 guard column (Thermo Scientific, USA). Separation of the nucleotides were carried out with a mobile phase containing $60 \mathrm{mmol} / \mathrm{L}$ of $\mathrm{KH}_{2} \mathrm{PO}_{4}, 0.45 \mathrm{mmol} / \mathrm{L}$ of tetrabutylammonium bromide $(\mathrm{TBAB})$ and $1.26 \mathrm{~mol} / \mathrm{L}$ of acetonitrile. The $\mathrm{pH}$ of the mobile phase was adjusted to 3.20 using concentrated phosphoric acid. Retention times of the individual nucleotides were 
matched to that of the respective nucleotide standards. Areas under the peaks for the respective nucleotides were tabulated and energy charge was calculated using the formula: Energy Charge $=[\mathrm{ATP}]+1 / 2[\mathrm{ADP}] /[\mathrm{ATP}]+[\mathrm{ADP}]+[\mathrm{AMP}]$

Kinase inhibitor array. MKN74 cells were treated with 95 different kinase inhibitors (SYN2103; Synkinase, Australia) in the absence and presence of rh-cANGPTL4 $(10 \mu \mathrm{g} / \mathrm{mL})$ for 6 h. RNA was isolated and reverse transcribed using the iScript Reverse Transcription Supermix. qPCR was done using the $\mathrm{KAPA}^{\mathrm{TM}}$ SYBR qPCR Universal Master Mix (KAPABiosystems).

Statistical analysis. Statistical analyses were performed using two-tailed Mann-Whitney test with SPSS software. All statistical tests were two-sided. $\mathrm{p}$ value of $\leq 0.05$ is considered significant.

Accession numbers. Microarray data have been submitted to the GEO database under accession number GSE 71280 


\section{Acknowledgements}

This work was supported by grants from Ministry of Education, Singapore (MOE2014-T2-1012 and 2012-T1-001-036). We thank Prof Walter Wahli (Lee Kong Chian School of Medicine, NTU), Prof Jeak Ling Ding (Department of Biological Sciences, NUS) and Dr

Carol Tang (National Neuroscience Institute, Singapore) for critical reading of the manuscript.

Contribution: ZT performed experiments, analyzed the results and wrote the article; MKS, JSKC, YL, MMKL, LL, TP and PZ performed experiments and contributed to discussion. NST analyzed the results, contributed to discussion, reviewed and edited the article.

Conflict-of-interest disclosure: The authors declare no competing financial interests. 


\section{References}

1. Steeg PS, Theodorescu D. Metastasis: a therapeutic target for cancer. Nat Clin Pract Oncol. 2008;5(4):206-219.

2. Siegel R, Ma J, Zou Z, Jemal A. Cancer statistics, 2014. CA Cancer J Clin. 2014;64(1):929.

3. Chiang AC, Massagué J. Molecular basis of metastasis. N Engl J Med. 2008;359(26):28142823.

4. Klymkowsky MW, Savagner P. Epithelial-mesenchymal transition: a cancer researcher's conceptual friend and foe. Am J Pathol. 2009;174(5):1588-1593.

5. Savagner P. Leaving the neighborhood: molecular mechanisms involved during epithelialmesenchymal transition. Bioessays. 2001;23(10):912-923.

6. Hanahan D, Weinberg RA. Hallmarks of cancer: the next generation. Cell. 2011;144(5):646-674.

7. Vander Heiden MG, Cantley LC, Thompson CB. Understanding the Warburg effect: the metabolic requirements of cell proliferation. Science. 2009;324(5930):1029-1033.

8. Ward PS, Thompson CB. Metabolic reprogramming: a cancer hallmark even warburg did not anticipate. Cancer Cell. 2012;21(3):297-308.

9. Dang CV, Semenza GL. Oncogenic alterations of metabolism. Trends Biochem Sci. 1999;24(2):68-72.

10. Dang CV, Lewis BC, Dolde C, Dang G, Shim H. Oncogenes in tumor metabolism, tumorigenesis, and apoptosis. J Bioenerg Biomembr. 1997;29(4):345-354.

11. Jones RG, Thompson CB. Tumor suppressors and cell metabolism: a recipe for cancer growth. Genes Dev. 2009;23(5):537-548.

12. Finger EC, Giaccia AJ. Hypoxia, inflammation, and the tumor microenvironment in metastatic disease. Cancer Metastasis Rev. 2010;29(2):285-293. 
13. Perl AK, Wilgenbus P, Dahl U, Semb H, Christofori G. A causal role for E-cadherin in the transition from adenoma to carcinoma. Nature. 1998;392(6672):190-193.

14. Thiery JP, Sleeman JP. Complex networks orchestrate epithelial-mesenchymal transitions. Nat Rev Mol Cell Biol. 2006;7(2):131-142.

15. Gonzalez DM, Medici D. Signaling mechanisms of the epithelial-mesenchymal transition. Science signaling. 2014;7(344):re8.

16. Zaravinos A. The Regulatory Role of MicroRNAs in EMT and Cancer. Journal of oncology. 2015;2015:865816.

17. Chong HC, Tan CK, Huang RL, Tan NS. Matricellular proteins: a sticky affair with cancers. Journal of oncology. 2012;2012:351089.

18. Lunt SJ, Chaudary N, Hill RP. The tumor microenvironment and metastatic disease. Clin Exp Metastasis. 2009;26(1):19-34.

19. Live TR, Kaminskas E. Changes in adenylate energy charge in Ehrlich ascites tumor cells deprived of serum, glucose, or amino acids. J Biol Chem. 1975;250(5):1786-1789.

20. Ridge JW. Hypoxia and the energy charge of the cerebral adenylate pool. Biochem J. 1972;127(2):351-355.

21. Atkinson DE, Walton GM. Adenosine triphosphate conservation in metabolic regulation. Rat liver citrate cleavage enzyme. J Biol Chem. 1967;242(13):3239-3241.

22. Hu H, Juvekar A, Lyssiotis CA, et al. Phosphoinositide 3-Kinase Regulates Glycolysis through Mobilization of Aldolase from the Actin Cytoskeleton. Cell. 2016;164(3):433-446. 23. Shiraishi T, Verdone JE, Huang J, et al. Glycolysis is the primary bioenergetic pathway for cell motility and cytoskeletal remodeling in human prostate and breast cancer cells. Oncotarget. 2015;6(1):130-143.

24. Szakács G, Paterson JK, Ludwig JA, Booth-Genthe C, Gottesman MM. Targeting multidrug resistance in cancer. Nat Rev Drug Discov. 2006;5(3):219-234. 
25. Liang XJ, Aszalos A. Multidrug transporters as drug targets. Curr Drug Targets. 2006;7(8):911-921.

26. Dang CV. Links between metabolism and cancer. Genes Dev. 2012;26(9):877-890.

27. Bridges D, Moorhead GBG. 14-3-3 proteins: a number of functions for a numbered protein. Sci STKE. 2005;2005(296):re10.

28. Goh YY, Pal M, Chong HC, et al. Angiopoietin-like 4 interacts with integrins beta1 and beta5 to modulate keratinocyte migration. Am J Pathol. 2010;177(6):2791-2803.

29. Tinti M, Madeira F, Murugesan G, Hoxhaj G, Toth R, Mackintosh C. ANIA: ANnotation and Integrated Analysis of the 14-3-3 interactome. Database : the journal of biological databases and curation. 2014;2014:bat085.

30. Mak BC, Yeung RS. The tuberous sclerosis complex genes in tumor development. Cancer Invest. 2004;22(4):588-603.

31. Cai SL, Tee AR, Short JD, et al. Activity of TSC2 is inhibited by AKT-mediated phosphorylation and membrane partitioning. J Cell Biol. 2006;173(2):279-289.

32. Hou Z, Peng H, White DE, et al. 14-3-3 binding sites in the snail protein are essential for snail-mediated transcriptional repression and epithelial-mesenchymal differentiation. Cancer Res. 2010;70(11):4385-4393.

33. Chaffer CL, Weinberg RA. A perspective on cancer cell metastasis. Science. 2011;331(6024):1559-1564.

34. Huang RL, Teo Z, Chong HC, et al. ANGPTL4 modulates vascular junction integrity by integrin signaling and disruption of intercellular VE-cadherin and claudin-5 clusters. Blood. 2011;118(14):3990-4002.

35. Tan MJ, Teo Z, Sng MK, Zhu P, Tan NS. Emerging roles of angiopoietin-like 4 in human cancer. Mol Cancer Res. 2012;10(6):677-688. 
36. Zhu P, Tan MJ, Huang RL, et al. Angiopoietin-like 4 protein elevates the prosurvival intracellular O2(-):H2O2 ratio and confers anoikis resistance to tumors. Cancer Cell. 2011;19(3):401-415.

37. Padua D, Zhang XHF, Wang Q, et al. TGFbeta primes breast tumors for lung metastasis seeding through angiopoietin-like 4. Cell. 2008;133(1):66-77.

38. Mhawech P. 14-3-3 proteins--an update. Cell Res. 2005;15(4):228-236.

39. Hermeking H. The 14-3-3 cancer connection. Nat Rev Cancer. 2003;3(12):931-943.

40. Freeman AK, Morrison DK. 14-3-3 Proteins: diverse functions in cell proliferation and cancer progression. Semin Cell Dev Biol. 2011;22(7):681-687.

41. Tzivion G, Gupta VS, Kaplun L, Balan V. 14-3-3 proteins as potential oncogenes. Semin Cancer Biol. 2006;16(3):203-213.

42. Mani SA, Guo W, Liao MJ, et al. The epithelial-mesenchymal transition generates cells with properties of stem cells. Cell. 2008;133(4):704-715.

43. Montagner A, Delgado MB, Tallichet-Blanc C, et al. Src is activated by the nuclear receptor peroxisome proliferator-activated receptor $\beta / \delta$ in ultraviolet radiation-induced skin cancer. EMBO molecular medicine. 2014;6(1):80-98.

44. Yang NC, Ho WM, Chen YH, Hu ML. A convenient one-step extraction of cellular ATP using boiling water for the luciferin-luciferase assay of ATP. Anal Biochem.

$2002 ; 306(2): 323-327$. 


\section{Figure Legends}

Figure 1. Altered cancer cell bioenergetics during EMT. (A, B, left panel) Immunofluorescence staining of E-cadherin in hypoxia- $(\mathbf{A})$ or 4-OHT-treated MKN74 ${ }_{\text {SnailER }}$ cells (B) at the indicated time intervals. Cells were counterstained with DAPI (blue) for nuclei and phalloidin (red) for actin cytoskeleton. Scale bar $=40 \mu \mathrm{m}$. (A, B, middle and right panels) Relative expression (middle) and immunodetection (right) of EMT markers in hypoxia-exposed MKN74 cells (A) or 4-OHT-treated MKN74 $4_{\text {SnaileR }}$ cells (B) at the indicated time intervals. Data (mean \pm SD) from three independent experiments. Loading controls are from the same sample. $* P<0.05, * * P<0.01$. (C) FACS analysis of fluorescent glucose analog, 2-NBDG, uptake in MKN74 ${ }_{\mathrm{CTRL}}$ and $\mathrm{MKN} 74_{\text {SnailER }}$ cells after the indicated treatments. (D) Glycolysis rate in MKN74 ${ }_{\text {CTRL }}$ cells after the indicated treatments. (E) In vivo 2-DG fluorescence imaging of mice bearing tumors that received the indicated treatments.

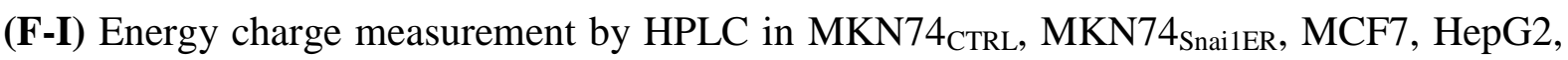
II4, and HSC cells (F, G), xenograft tumors $(\mathbf{H})$ and tumor biopsies from patients (I) after the indicated treatments. veh: vehicle

Figure 2. Metabolic reprogramming enhanced metastatic capability of cancer cells. (A-

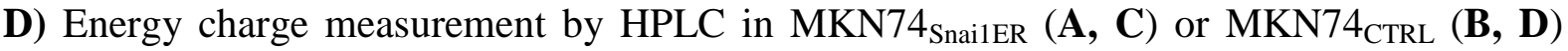
cells harboring the respective modification after the indicated treatments. * $P<0.05$; ** $P<0.01$. (E, F) Immunofluorescence staining of E-cadherin in MKN74 cells with the indicated modification under normoxic or hypoxic conditions. Nuclei were counterstained with DAPI (blue). Scale bar $=50 \mu \mathrm{m}$. (G) Representative immunofluorescence images of Ecadherin and laminin 332 from MKN74 SnailER tumor sections exposed to the indicated treatments. Scale bar $=40 \mu \mathrm{m}$ (top) or $100 \mu \mathrm{m}$ (bottom). (H) Gelatin zymography and quantification of MMP activities in the indicated tumors. (I) Representative macroscopic images of lungs and the relative expression of human TBP from the indicated lungs. Black 
arrows in (I) indicate micrometastases $(\mathbf{n = 5})$. (J, K) FACS analysis of fluorescent dye efflux (J) and Annexin/PI (K) staining in MKN74 ${ }_{\text {CTRL }}$ and MKN74 $4_{\text {SnailER }}$ cells after the indicated treatments. (L, M) Immunodetection of MDR1 in MKN74 $4_{\mathrm{CTRL}}, \mathrm{MKN74} 4_{\text {SnaileR }}$ cells $(\mathbf{L})$ and tumor biopsies from patients (M) after indicated treatment.

Figure 3. ANGPTL4 regulates cellular bioenergetics during EMT. (A, left panel) Top five most represented biological processes in hypoxia (top), TGF- $\beta$ (middle), and 4-OHT (bottom) induced EMT models. (A, right panel) Top five most enriched metabolic processes. (B) Heat map representation of commonly regulated genes across the three EMT models (control vs. ANGPTL4-knockdown) over the indicated time points. Green: downregulated; Red: up-regulated; White box: groups of genes that are differentially regulated in ANGPTL4-knockdown cells compared to control cells. (C) Relative expression of selected genes involved in the four enriched metabolic processes. (D) Total ROS content of MKN74 or MKN74 ${ }_{\text {SnailER }}$ cells during EMT based on fluorescence measurements. Loading controls are from the same sample. (E-G) Immunodetection of ANGPTL4 in MKN74 CTRL, $_{\text {, }}$ MKN74 $_{\text {SnailER }}$ cells (E), xenograft tumors (F) and tumor biopsies from patients $(\mathbf{G})$ after indicated treatment. Loading controls are from the same sample. (H-L) Energy charge measurement by HPLC in MKN74 $4_{\text {CTRL }}, \mathrm{MKN} 4_{\text {SnailER }}, \mathrm{MCF} 7, \mathrm{HepG} 2$, II4, and HSC cells $(\mathbf{H}-\mathbf{K})$ and xenograft tumors $(\mathbf{L})$ after the indicated treatments. veh: vehicle; Ab: $\alpha-$ cANGPTL4; Kd: ANGPTL4-knockdown.

Figure 4. Metabolic reprogramming by ANGPTL4 augments EMT. (A-C, top panel) Immunofluorescence staining of E-cadherin in hypoxia- (A) or rh-cANGPTL4- (C) treated MKN74 cells or 4-OHT-treated MKN74 $4_{\text {SnailER }}$ cells (B) in the presence of $\alpha$-cANGPTL4 at the indicated time intervals. Cells were counterstained with DAPI (blue) for nuclei and phalloidin (red) for actin cytoskeleton. Scale bar $=40 \mu \mathrm{m}$. (A-C, bottom panels) Immunodetection of EMT markers in hypoxia-exposed MKN74 or MKN74 ${ }_{\triangle A N G P T L 4}$ cells 
(A), rh-cANGPTL4-treated MKN74 cells (C), or 4-OHT-treated MKN74 $4_{\text {SnailER }}$ cells (B) exposed to $\alpha$-cANGPTL4 at the indicated time intervals. Data (mean \pm SD) from three independent experiments. $* P<0.05, * * P<0.01$. Loading controls are from the same sample. (D) Gelatin zymography and quantification of MMP activities in the indicated tumors. (E) Representative macroscopic images of lungs and the relative expression of human TBP from the indicated lungs. Black arrows in (E) indicate micrometastases $(n=5)$. Immunofluorescence staining of cANGPTL4 and E-cadherin in benign and metastatic skin tumors. Scale bar $=100 \mu \mathrm{m}$. $(\mathbf{G}, \mathbf{H})$ FACS analysis of fluorescent dye efflux MKN74 $\mathrm{CTRL}$, MKN74 $_{\triangle \text { ANGPTL4 }}(\mathbf{G})$ and MKN74 $4_{\text {SnailER:shANGPTL4 }}(\mathbf{H})$ cells after the indicated treatments. (I) Immunodetection of MDR1 in MKN74 $4_{\text {CTRL }}$, MKN74 ${ }_{\triangle A N G P T L 4}$ and MKN744 $4_{\text {SnailER:shANGPTL4 }}$ cells after indicated treatment.

Figure 5. ANGPLT4 regulates specific 14-3-3 expression. (A-C) Immunodetection of various 14-3-3 isoforms in MKN74 $4_{C T R L}, M_{K N 74_{\triangle A N G P T L 4} \text {, and MKN74 }}$ SnailER cells under hypoxia- (A) and 4-OHT-induced (B) EMT or in the presence of rh-cANGPTL4 (C) at the indicated time intervals. (D) Analysis map of kinases (solid line) whose inhibition attenuates ANGPTL4-induced 14-3-3 expression and possible downstream transcriptional regulators (dotted line). (E-J) Immunodetection of the indicated proteins in hypoxia-treated MKN744 $_{\text {CTRL }}$ or MKN74 ${ }_{\triangle \mathrm{ANGPTL}}$ cells $(\mathbf{E}, \mathbf{J})$, 4-OHT-treated MKN74 $4_{\text {SnaileR }}$ cells $(\mathbf{F})$, rhcANGPTL4-treated MKN74 $4_{\text {CRL }}$ cells $(\mathbf{G}, \mathbf{J})$, xenograft tumors $(\mathbf{H})$, and tumor biopsies from patients (I) at the indicated time points. Loading controls are from the same sample.

Figure 6.The ANGPTL4:14-3-3 $\gamma$ axis coordinates energy demand during EMT. (A, E) Immunodetection of indicated 14-3-3 isoforms with $\mathrm{TSC}_{\mathrm{S} 939}$ (A) or Snai1 (E) after immunoprecipitation with Snai1, TSC2, or pan 14-3-3 antibodies. (B-D and F-H) Proximity

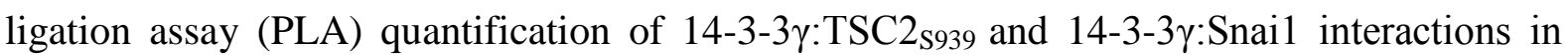


MKN74 $_{\mathrm{CTRL}}$ and MKN74 ${ }_{\triangle \mathrm{ANGPTL}}$ cells $(\mathbf{B}, \mathbf{F})$, xenograft tumors $(\mathbf{C}, \mathbf{G})$, and tumor biopsies from patients $(\mathbf{D}, \mathbf{H})$ after the indicated treatments. Data (mean \pm SD) from three independent experiments. $* P<0.05, * * P<0.01$. (I) Representative ChIP results using anti-Snai1 (top

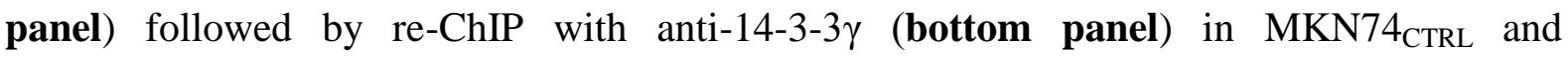
MKN74 $_{\triangle A N G P T L 4}$ cells under normoxic or hypoxic conditions. Pre-immune serum (IgG) served as control. (J) Schematic diagram depicting the coordination of metabolic reprogramming by the ANGPTL4:14-3-3 axis during EMT. 

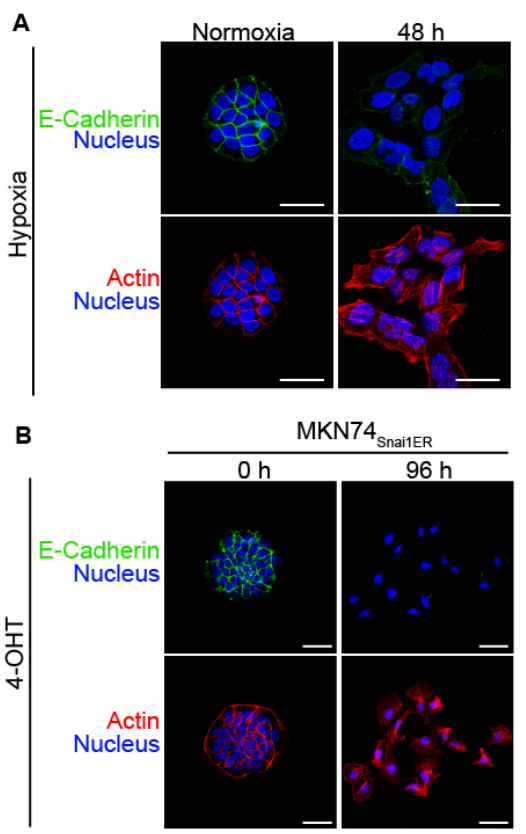

C
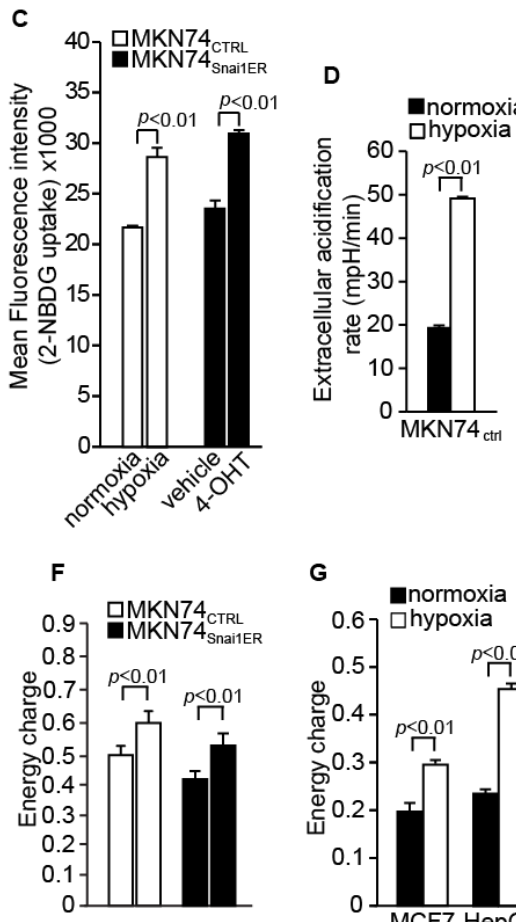
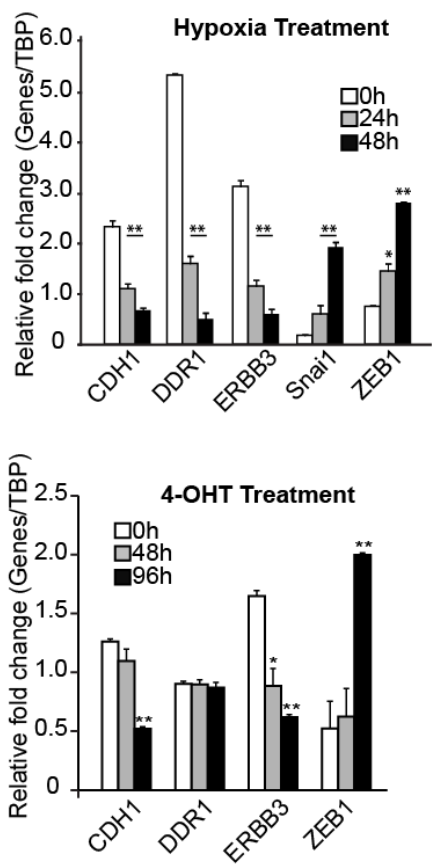

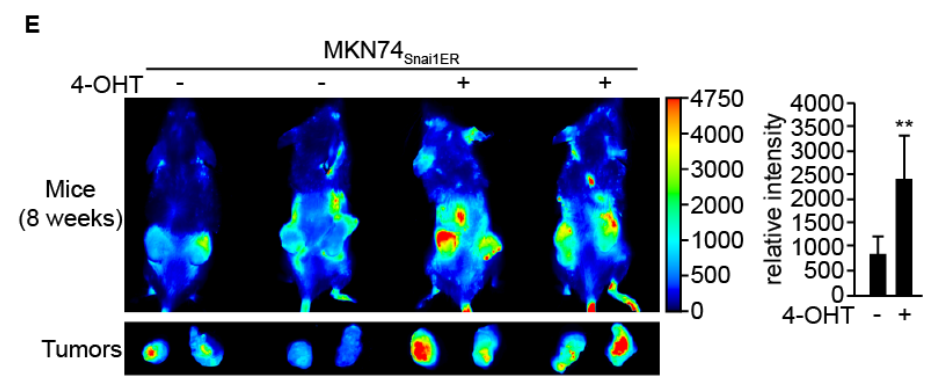

G

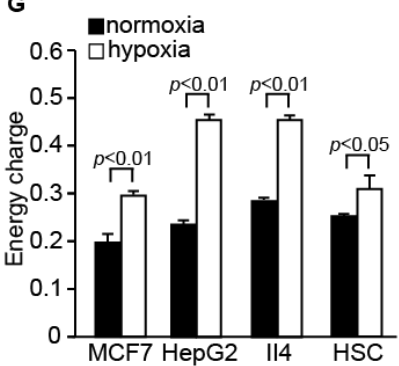

H

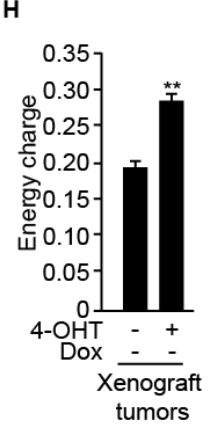

Hypoxia exposure $\overline{0 \mathrm{~h} 24 \mathrm{~h} 48 \mathrm{~h}} \mathrm{kDa}$ E-cadherin $=-140$
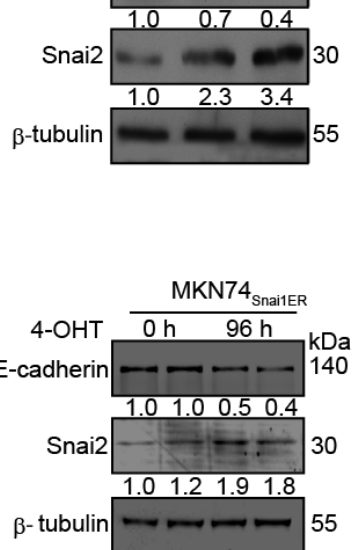

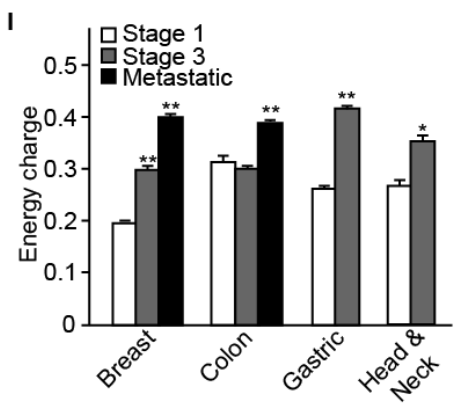



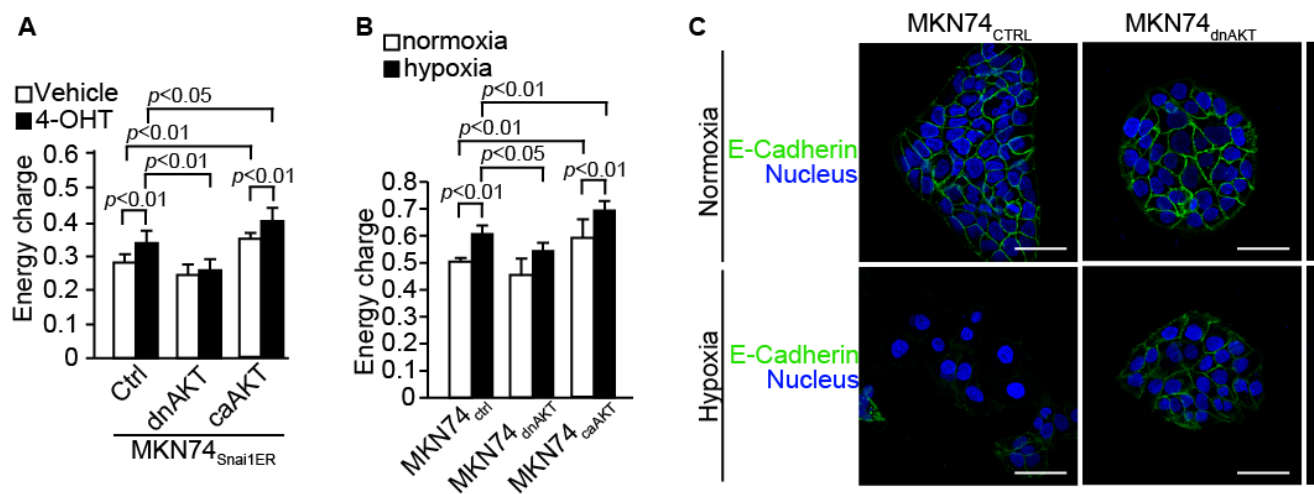

D
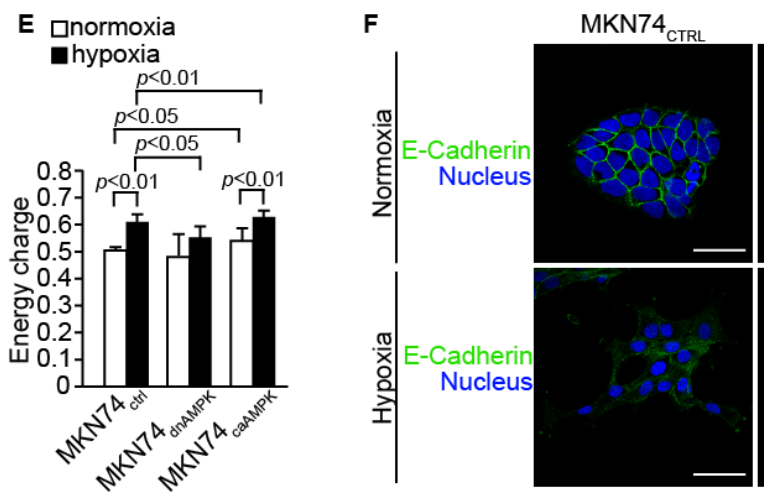

MKN74
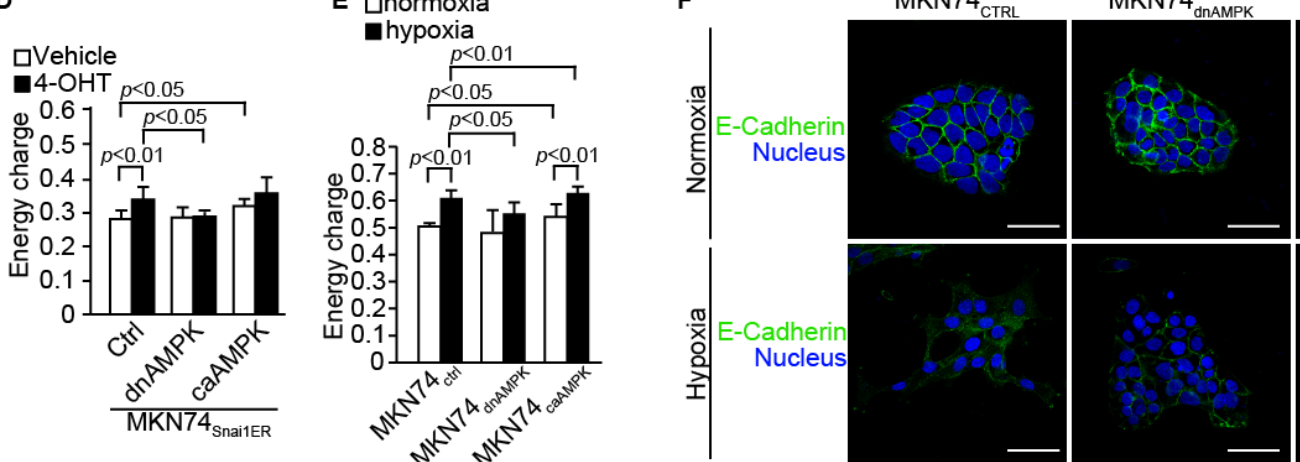

MKN74
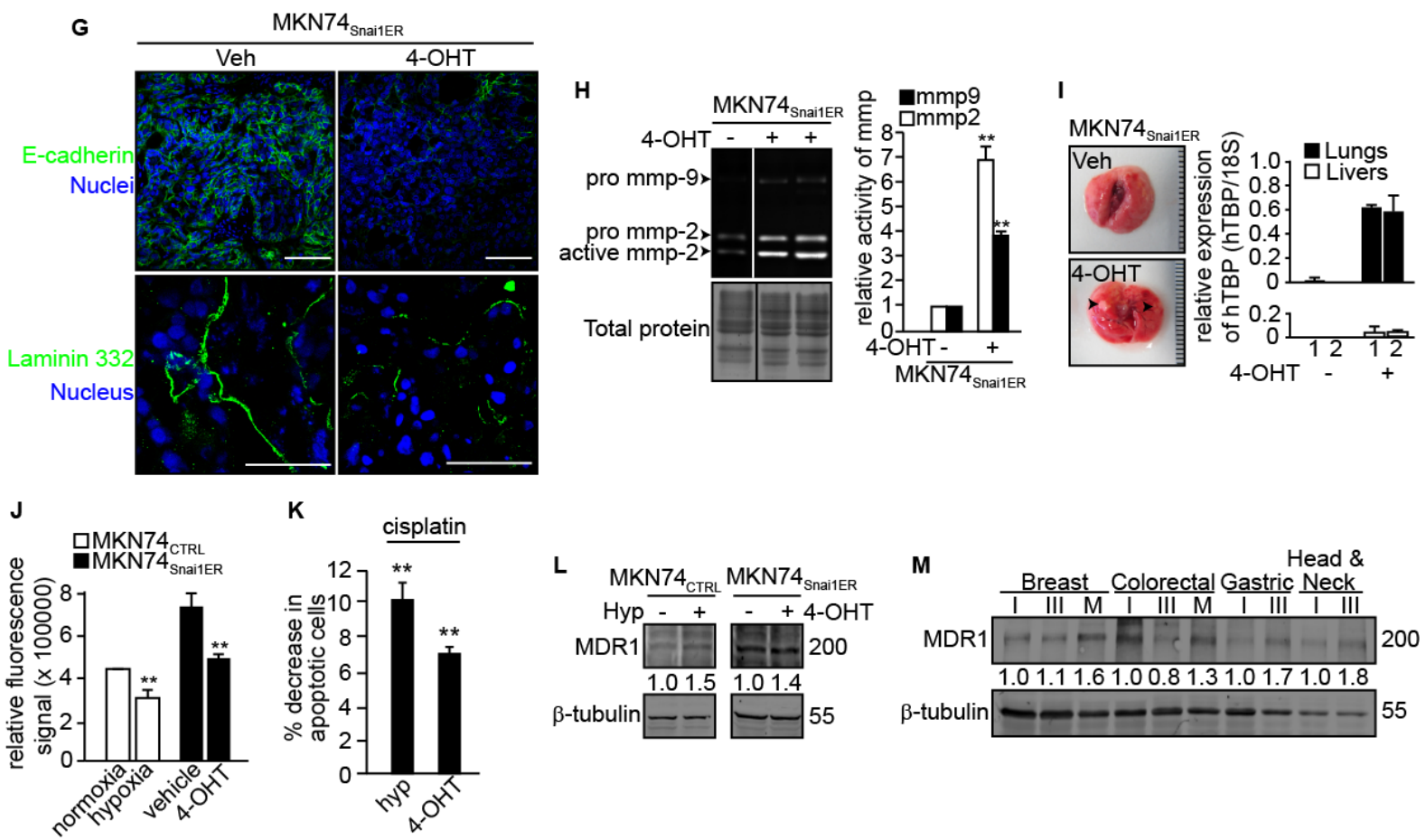

K

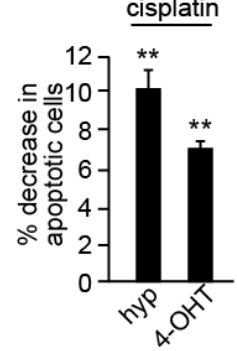

L MKN74 $4_{\text {CTRL }}$ MKN74

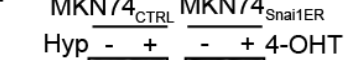

MDR1 $\square 200$

$\beta$-tubulin 1.01 .51 .01 .4

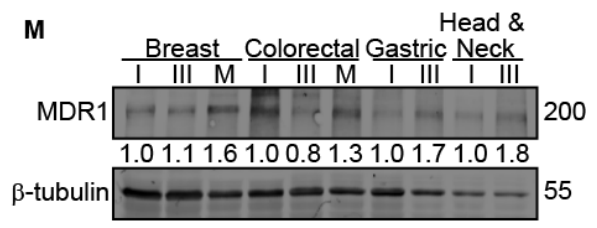


Teo et al Figure 3

\section{A Hypoxia-induced EMT}

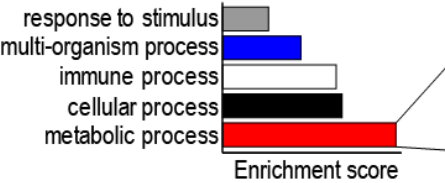

F A metabolic process glucose metabolic process ROS metabolic process

autophagy A.A metabolic process

TGF- $\beta$ induced EMT response to stimulus $\square$ cellular process

immune process metabolic process multi-organism process

\section{Enrichment score}

4-OHT induced EMT

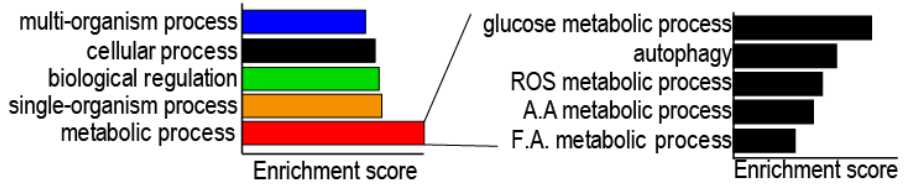

B
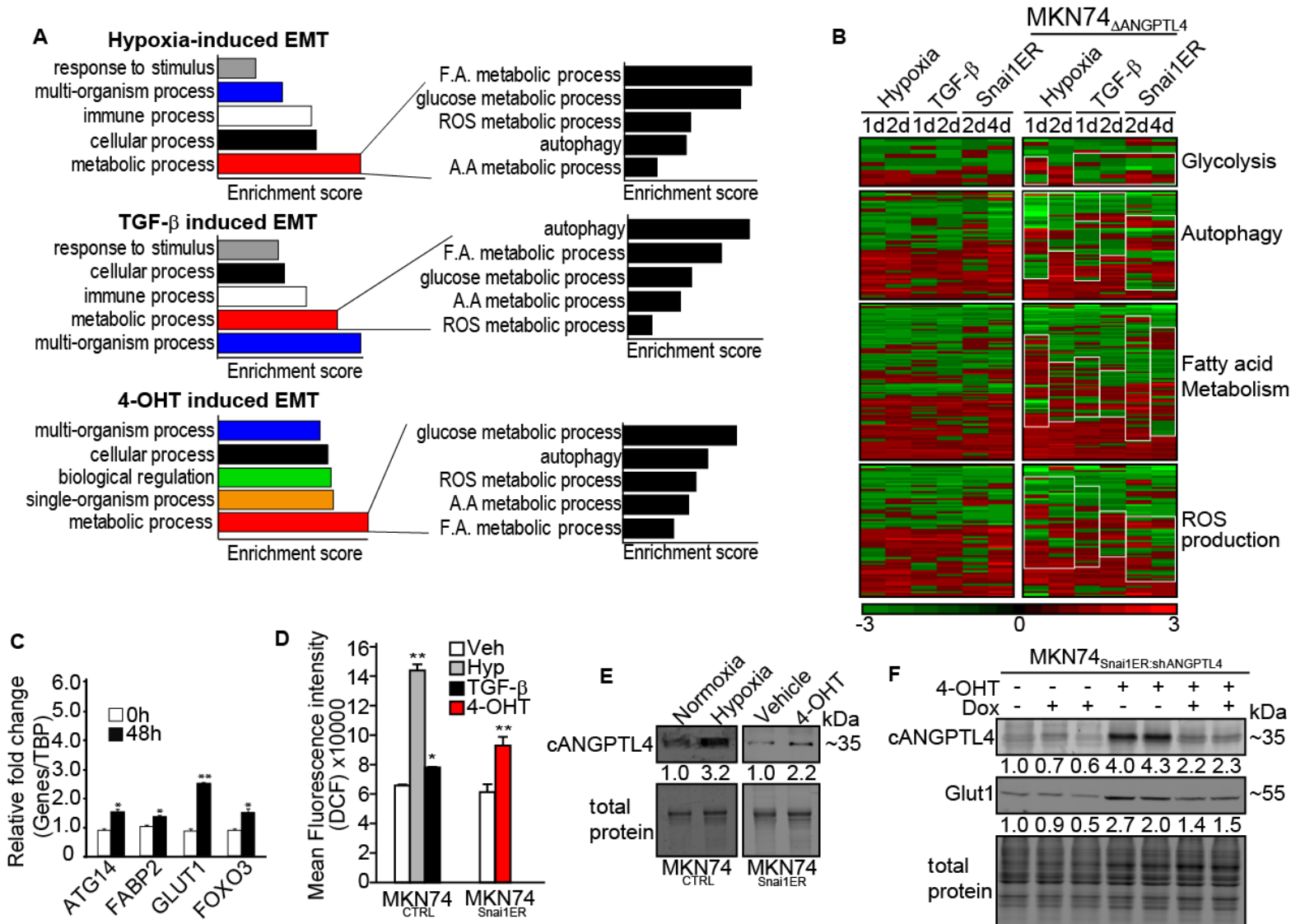

G
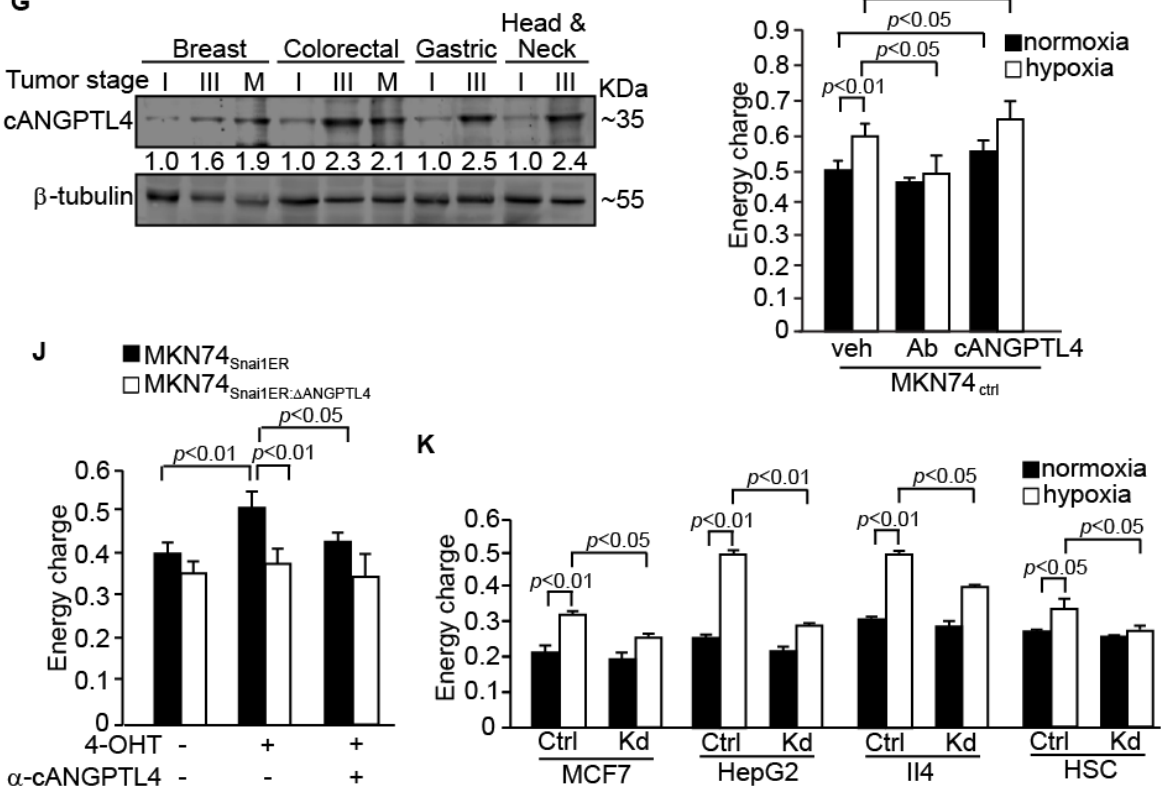
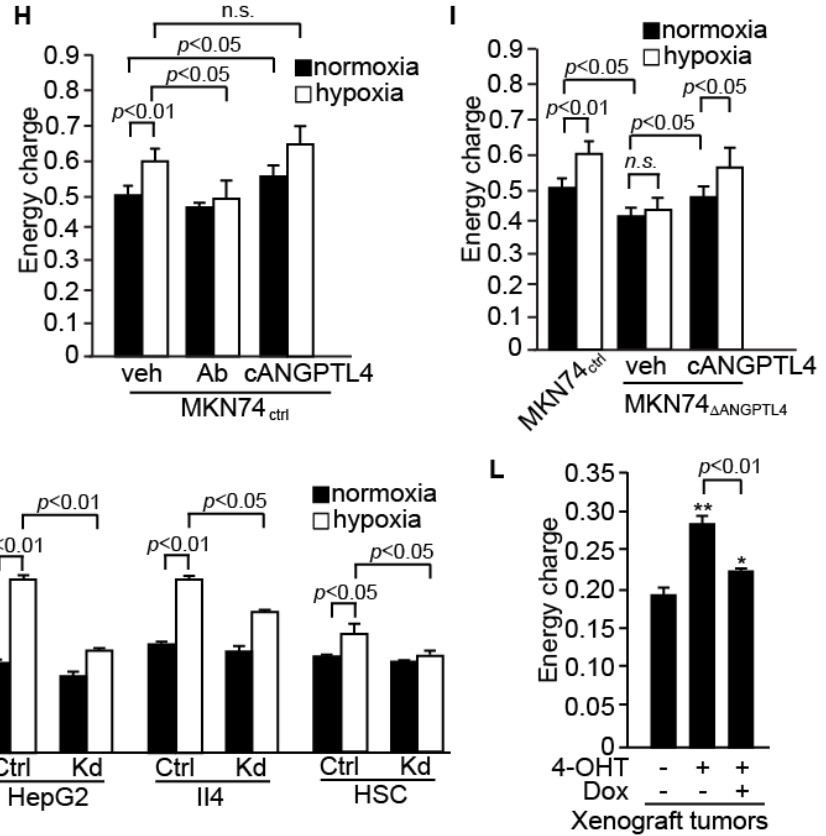
Teo et al Figure 4

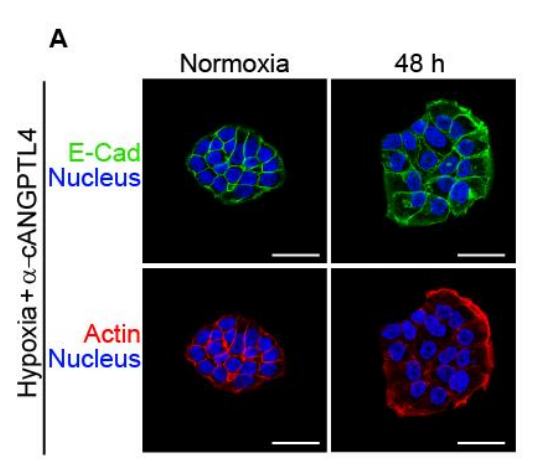

$+\alpha$-cANGPTL4 $\quad$ MKN74 $\triangle$ ANGPTL4
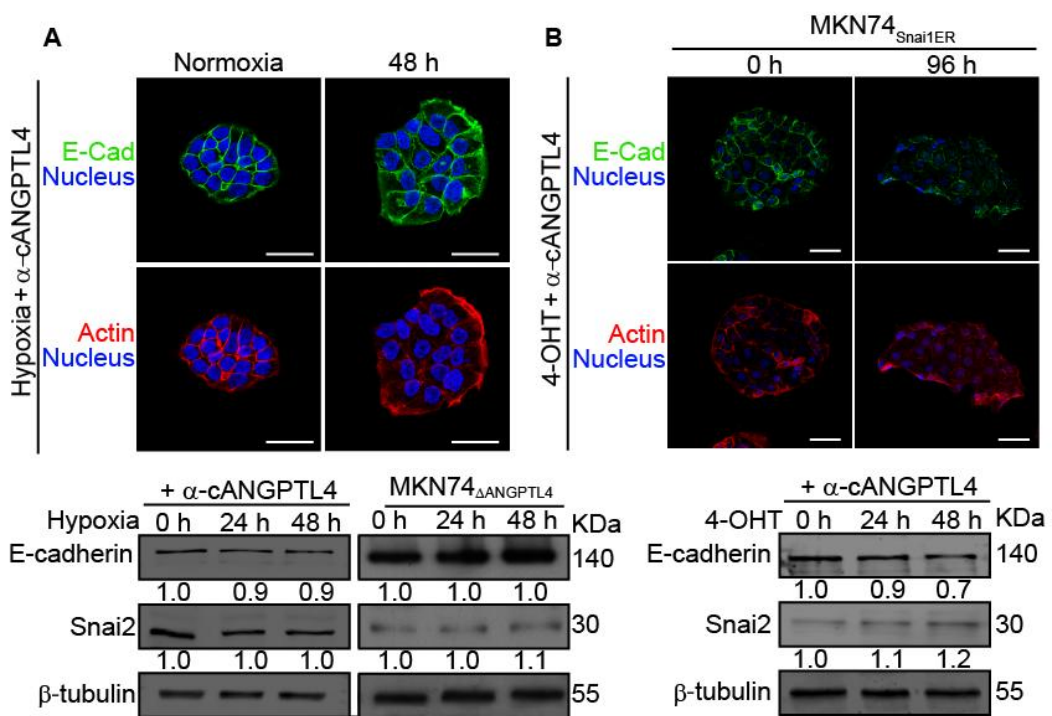

c
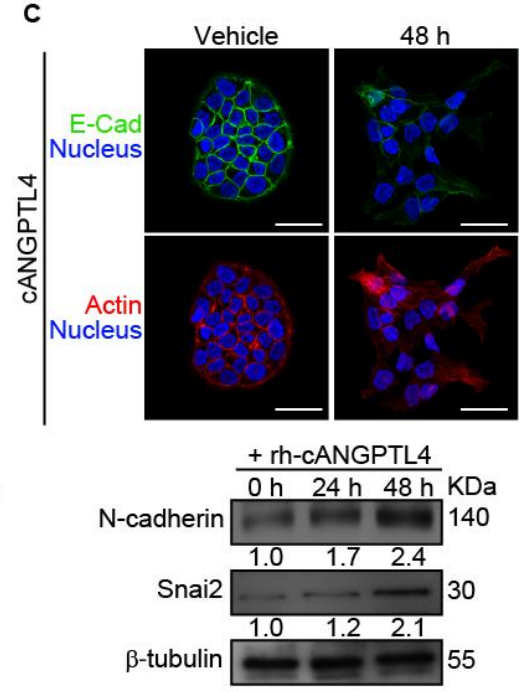
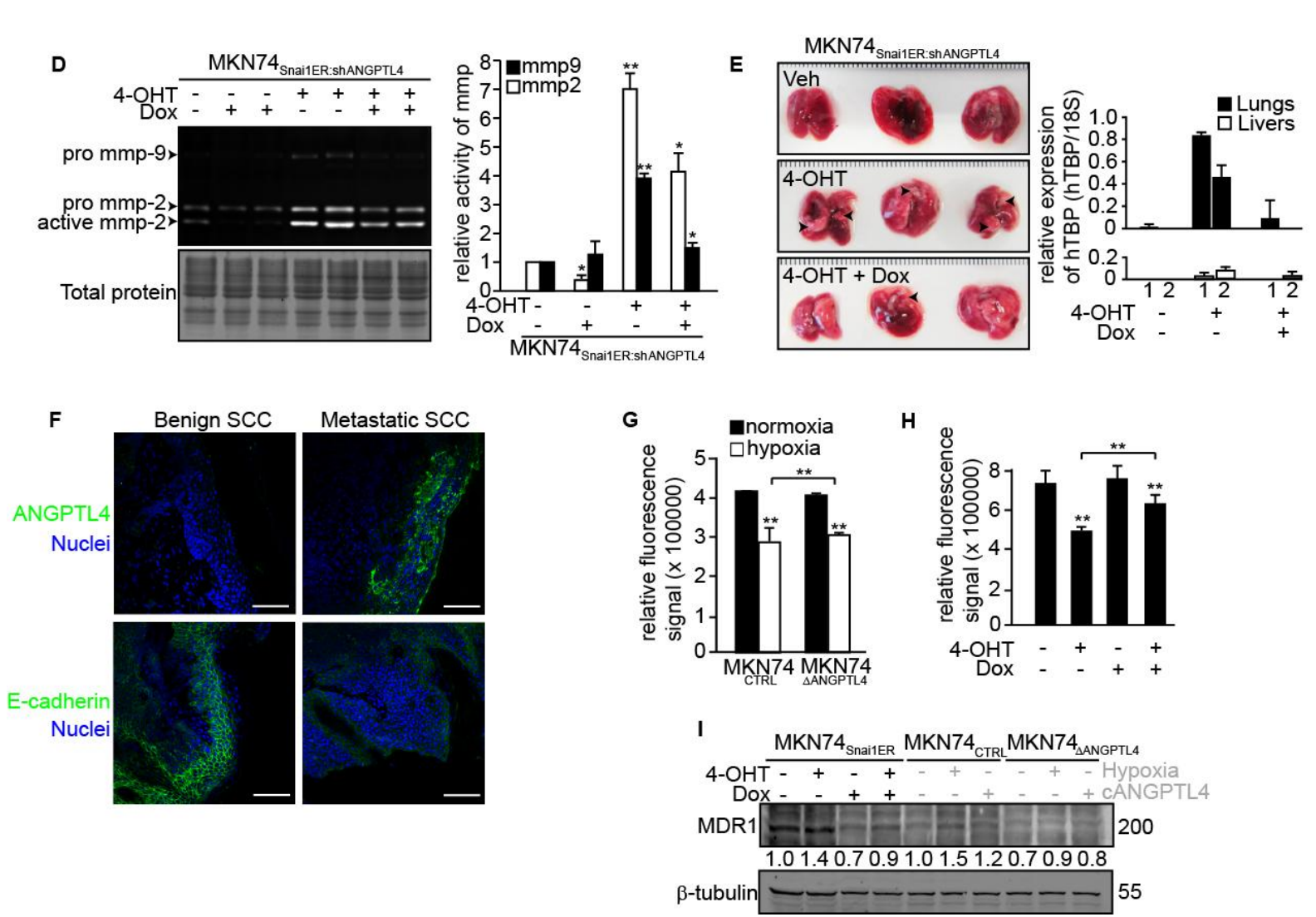

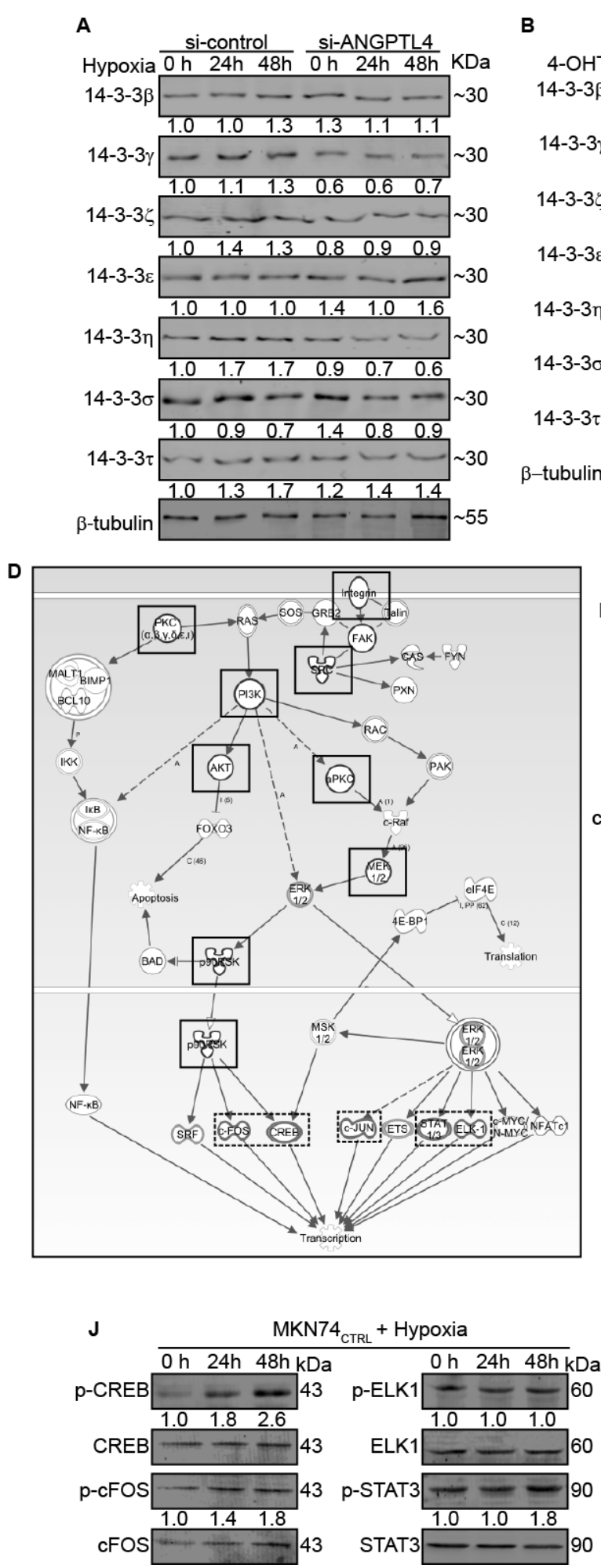

B 4 -OHT $\frac{\text { MKN74 }_{\text {Snai1ER }}}{0 h 48 \text { h } 96 \mathrm{~h}} \mathrm{KDa}$ 14-3-3 $-\div-\sim 30$

$$
\begin{array}{lll}
1.0 & 1.4 & 3.3 \\
\hline
\end{array}
$$

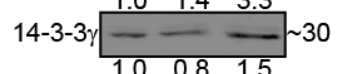

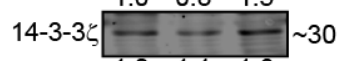$$
14-3-3 \varepsilon+1.0 \quad 1.1 \quad 1.6
$$$$
\begin{array}{llll}
14-3-3 \eta & 1.2 \quad 1.8 \\
\hline & - & - & -
\end{array}
$$

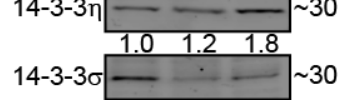

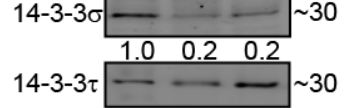$$
\begin{array}{lll}
1.0 & 1.4 \quad 2.9 \\
\beta-\text { tubulin } & - & -
\end{array}
$$

c $\quad \mathrm{MKN}_{\mathrm{CTRL}}$

CANGPTL4 0 h $24 \mathrm{~h} 48 \mathrm{~h}$ KDa

14-3-3 $-\square \sim 30$

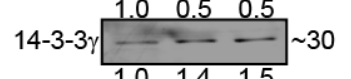$$
\text { 14-3-3ל } \begin{array}{llll}
1.0 & 1.4 & 1.5 \\
\hline & & & - \\
\hline
\end{array}
$$

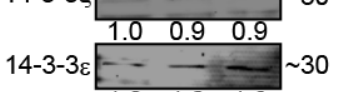$$
\begin{array}{llll}
1.0 & 1.2 & 1.8 \\
14-3-3 \eta & - & - & \cdots
\end{array}
$$

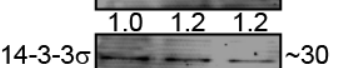$$
\begin{array}{llll}
1.0 & 0.9 & 0.4 \\
14-3-3 \tau & \ldots & \ldots &
\end{array}
$$$$
\beta \text {-tubulin } \begin{array}{lll}
1.0 & 1.2 & 0.9 \\
- & - & -
\end{array} 55
$$

E

si-control si-ANGPTL4 Hypoxia $0 \mathrm{~h} \mathrm{24h48 \textrm {h }} 0 \mathrm{~h} \mathrm{24h48 \textrm {h }} \mathrm{KDa}$ p-AKT $(\mathrm{S} 473) \cdots \cdots \cdots \cdots$

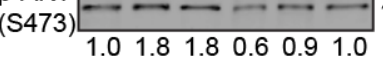
АKT $\longrightarrow+\cdots+70$

G $\quad$ MKN74 CANGPTL4 $\frac{\text { MKN74 }}{\text { CTRL } 24 \mathrm{~h} 48 \mathrm{~h}}$ KDa p-AKT
$(\mathrm{S} 473)$

$$
\begin{array}{llll}
1.0 & 1.42 .1 \\
\hline & &
\end{array}
$$
AKT $\longrightarrow 70$

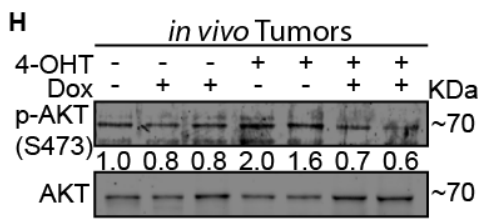

I

Head \&

Breast Colorectal Gastric Neck

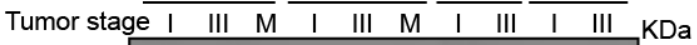

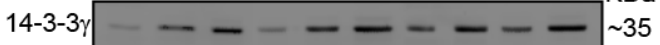
$\begin{array}{lllllllllll}1.0 & 1.7 & 2.1 & 1.0 & 1.9 & 2.1 & 1.0 & 2.1 & 1.0 & 2.3\end{array}$ $\beta$-tubulin $\longrightarrow-\ldots--\infty-\infty-\infty$
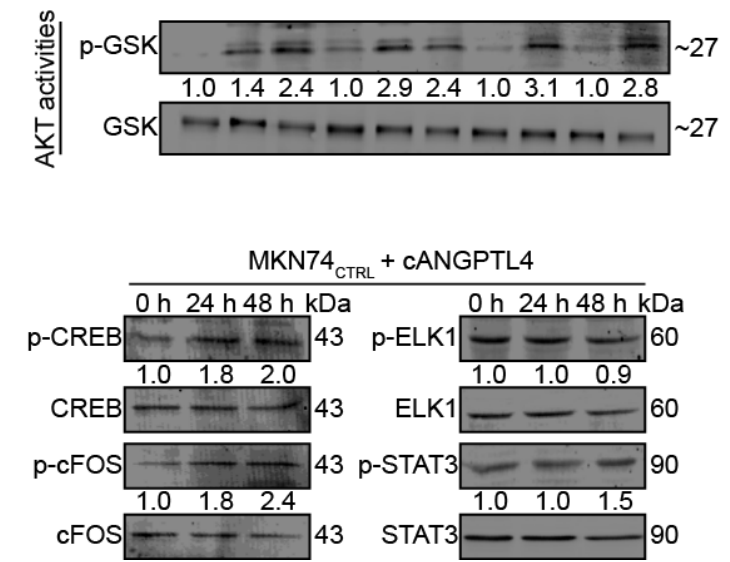
A

IP: TSC2 si-control si-ANGPTL4

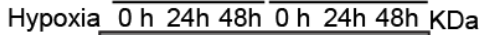
IB:TSC2 - - - - 289 IB:p-TSC2 … 289 (S-939) $\begin{array}{llllll}1.0 & 1.3 & 1.7 & 0.8 & 0.8 & 0.9\end{array}$

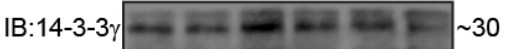
$\begin{array}{llllll}1.0 & 0.9 & 1.4 & 0.7 & 0.7 & 0.6\end{array}$
B

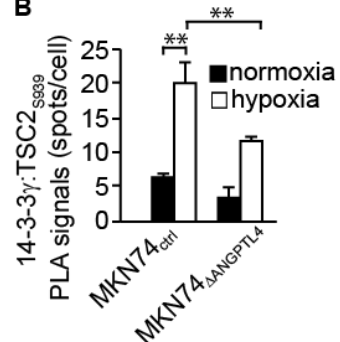

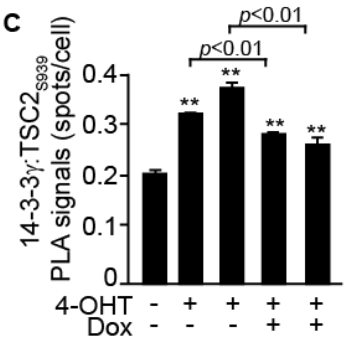

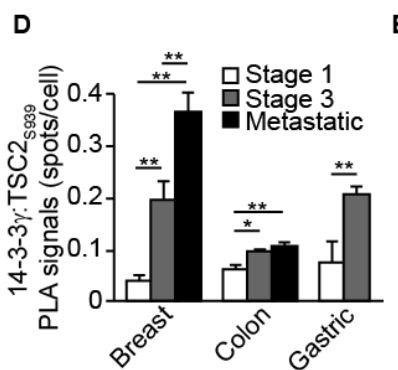

E IP: 14-3-3 si-control si-ANGPTL4 Hypoxia 0 h 24h 48h Oh 24h 48h KDa

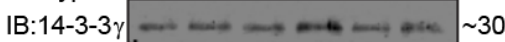

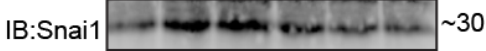

IP: Snai1 si-control si-ANGPTL4

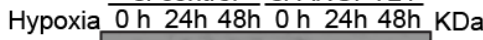
IB:Snai

IB:14-3-3

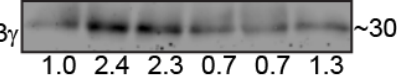

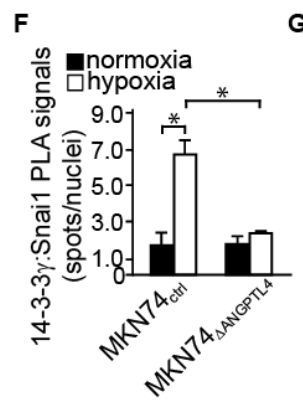

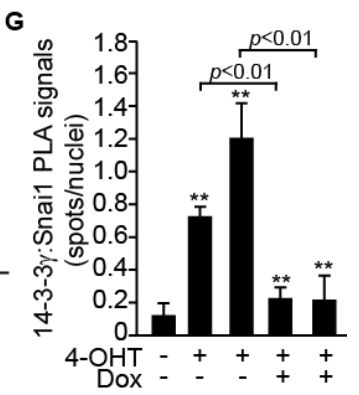

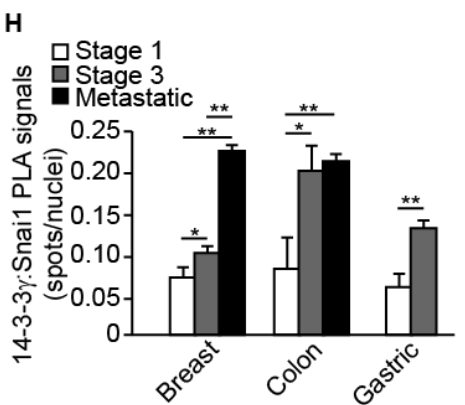

Snai1 binding site

Negative Control
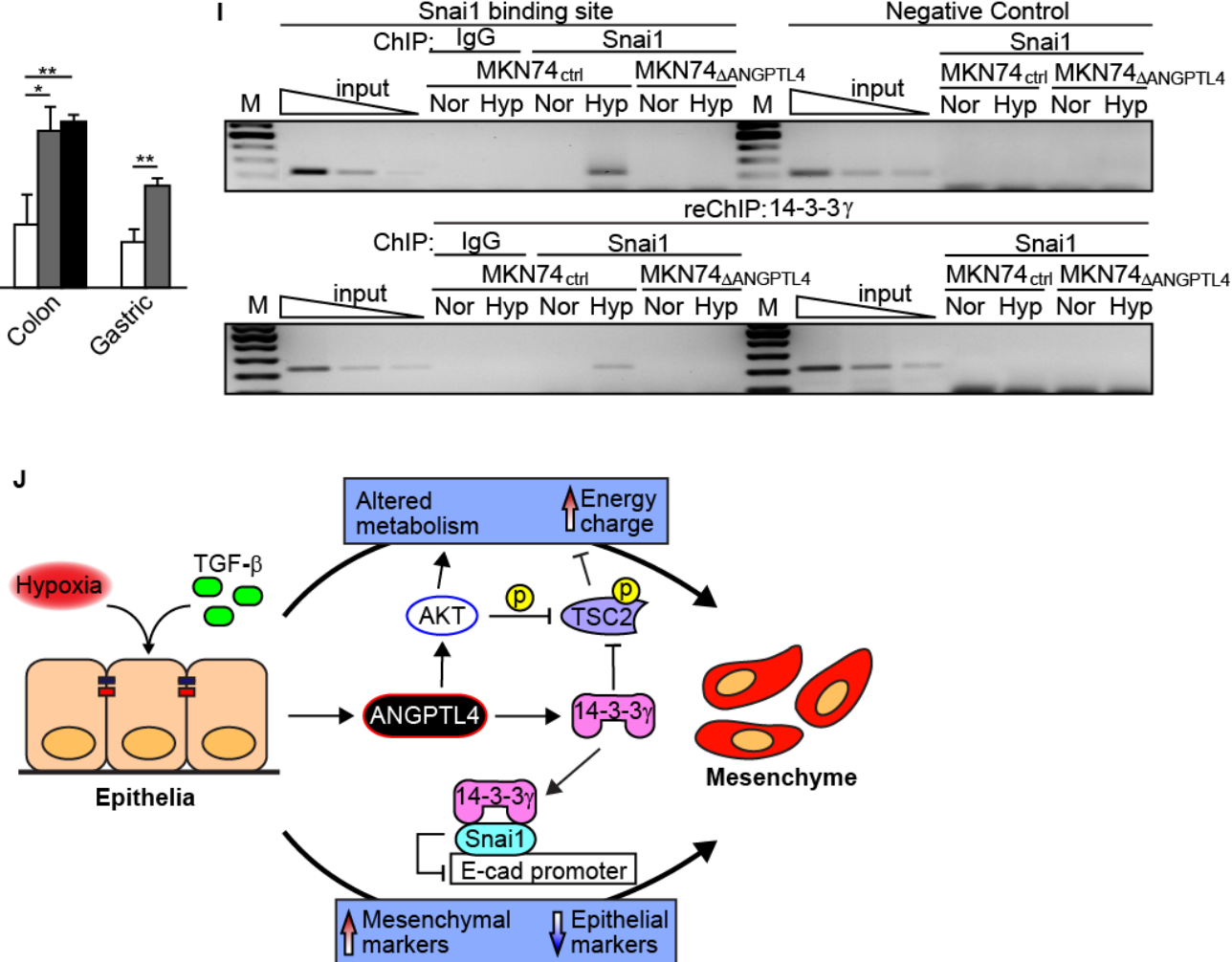



\section{SUPPLEMENTAL INFORMATION}

Reprogramming of cellular Bioenergetics Primes cancer cells for EpithelialMesenchymal Transition

Ziqiang TEO ${ }^{1}$, Ming Keat $\mathrm{SNG}^{1}$, Jeremy Soon Kiat $\mathrm{CHAN}^{1}$, Yinliang $\mathrm{LI}^{1}$, Liang $\mathrm{LI}^{1}$, Maegan Miang Kee LIM $^{1}$, Terri PHUA ${ }^{1,2}$, Pengcheng ZHU ${ }^{1}$, Nguan Soon TAN ${ }^{1,3,4}$

${ }^{1}$ School of Biological Sciences, Nanyang Technological University, 60, Nanyang Drive, Singapore 637551.

${ }^{2}$ Karolinska Institutet, Department of Microbiology, Tumor and Cell Biology. Nobels väg 16, Stockholm 17177, Sweden

${ }^{3}$ Institute of Molecular and Cell Biology, 61 Biopolis Drive, Proteos, Singapore 138673.

${ }^{4}$ KK Research Centre, KK Women’s and Children’s Hospital, 100 Bukit Timah Road, Singapore 229899

Correspondence:

PZ, email: PCZhu@ntu.edu.sg; ZT, email: zqteo@ntu.edu.sg

NST (senior corresponding author), email: nstan@ntu.edu.sg, Tel: +65-6316-2941, Fax: +6567913856 

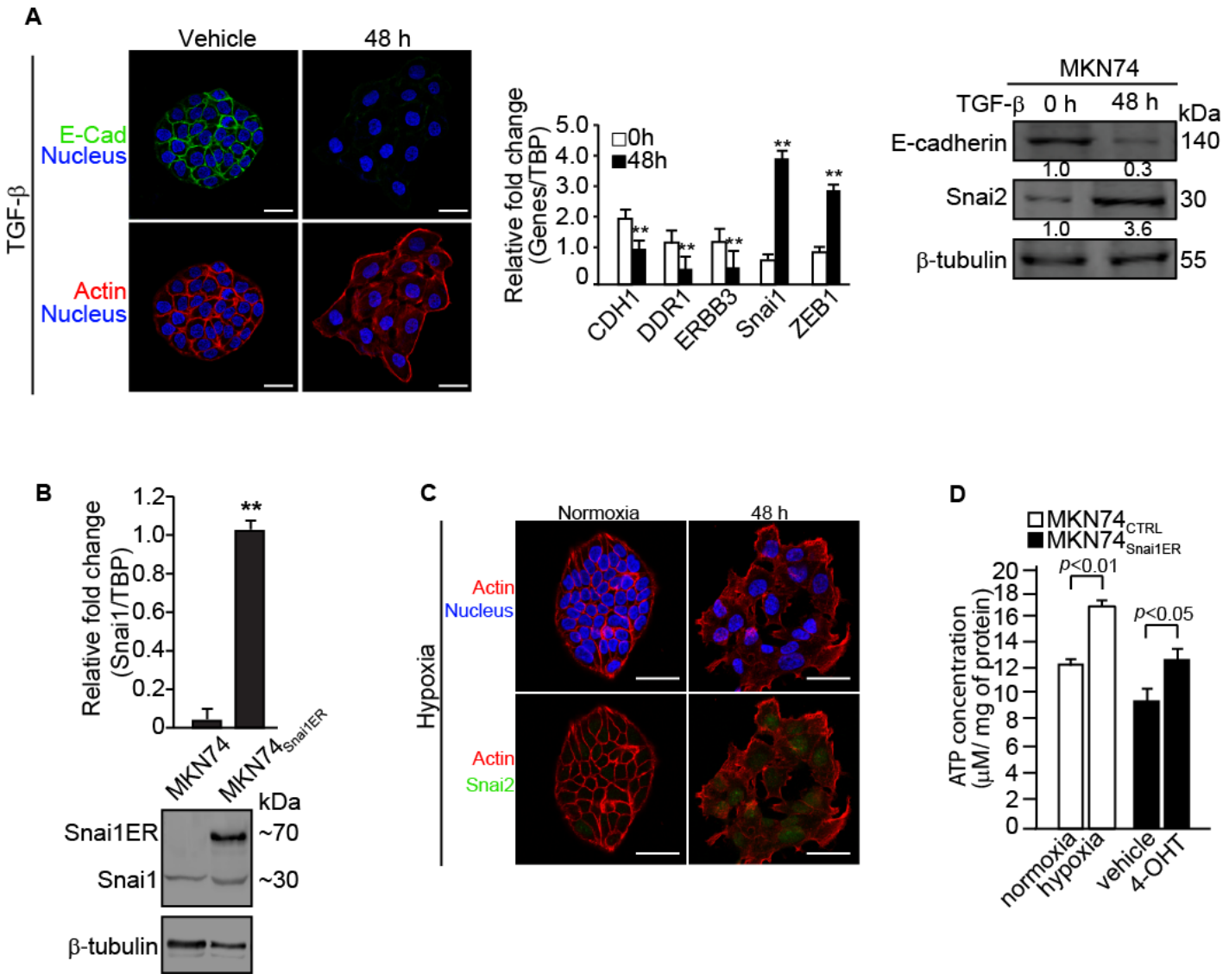
A
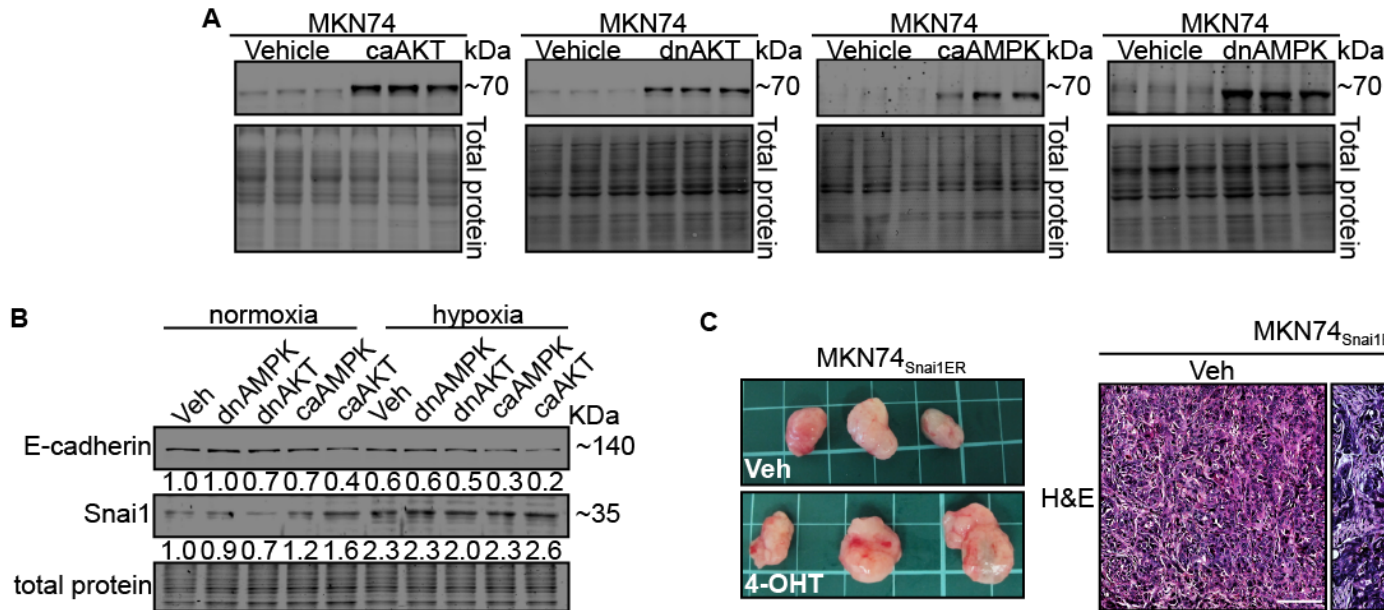

C
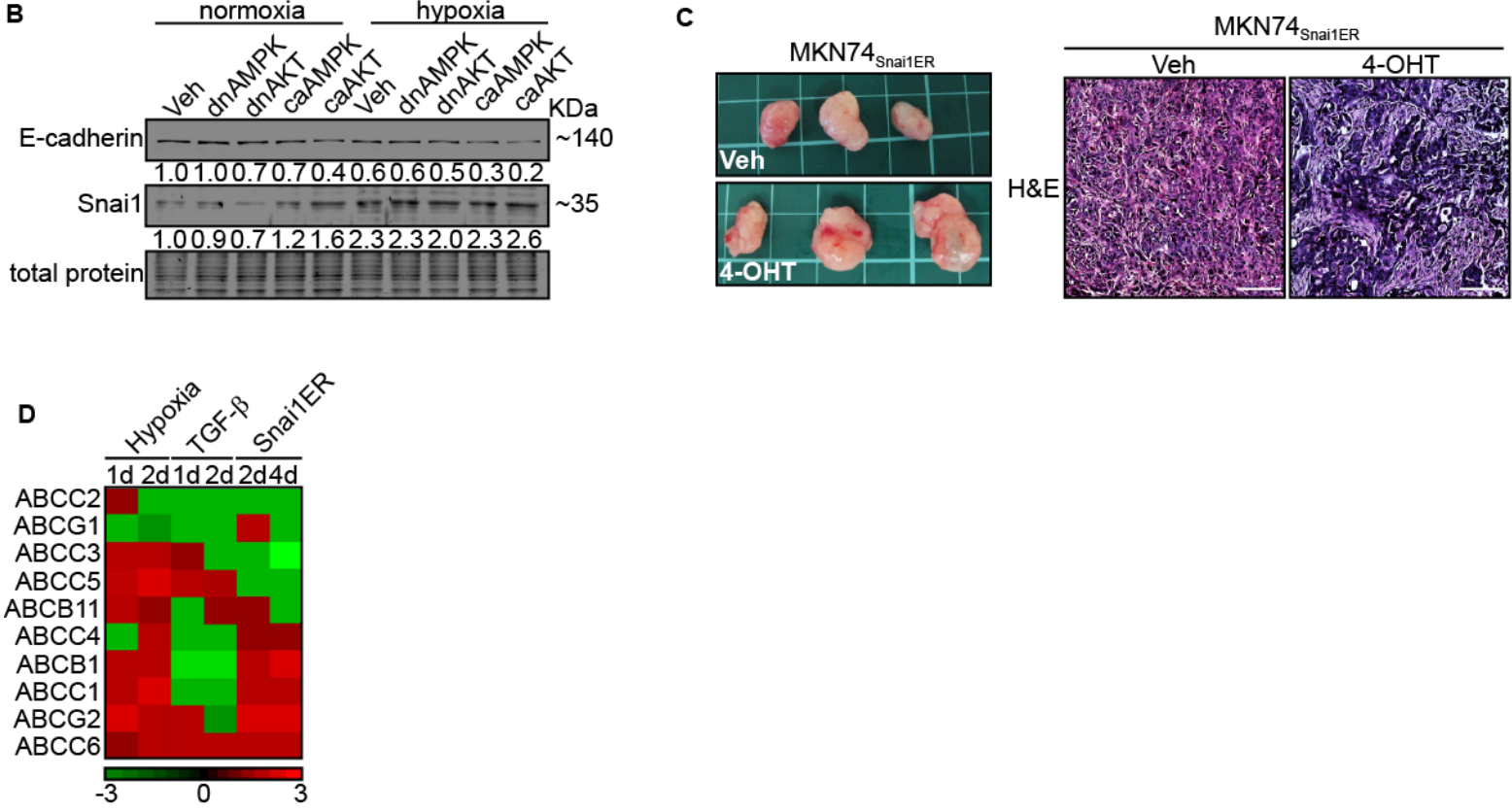
A

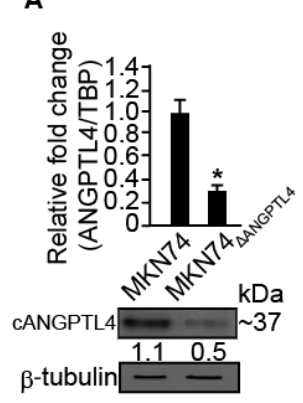

B si-ctrl si-ANGPTL4 Hypoxia $\frac{\text { si-ctrl }}{0 \mathrm{~h} 48 \mathrm{~h}}$ Si-AN $48 \mathrm{~h}$ KDa LC3II $\sum_{\beta} \begin{gathered}1.01 .81 .20 .9 \\ 1.02 .61 .211 .0\end{gathered}$ a) 4-OHT Oh 48h 96h LC3I,

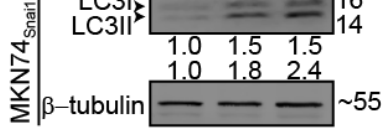

c

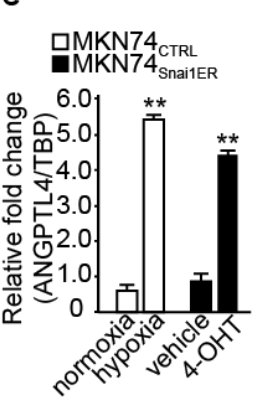

D

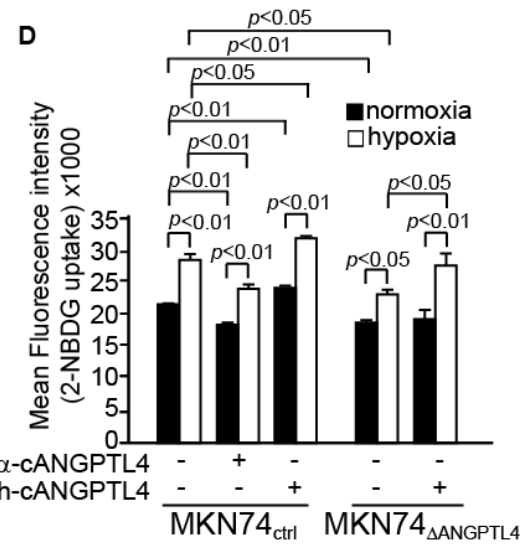

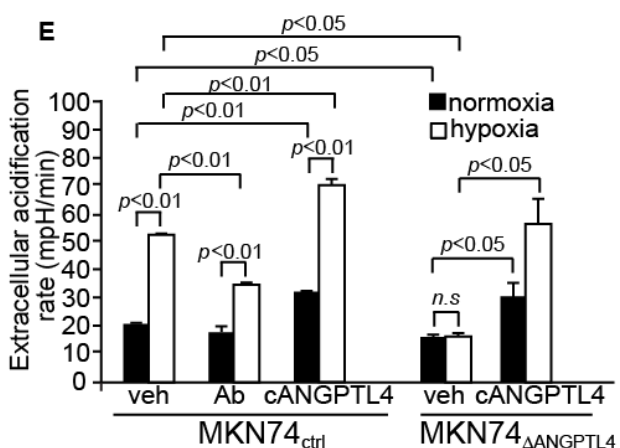
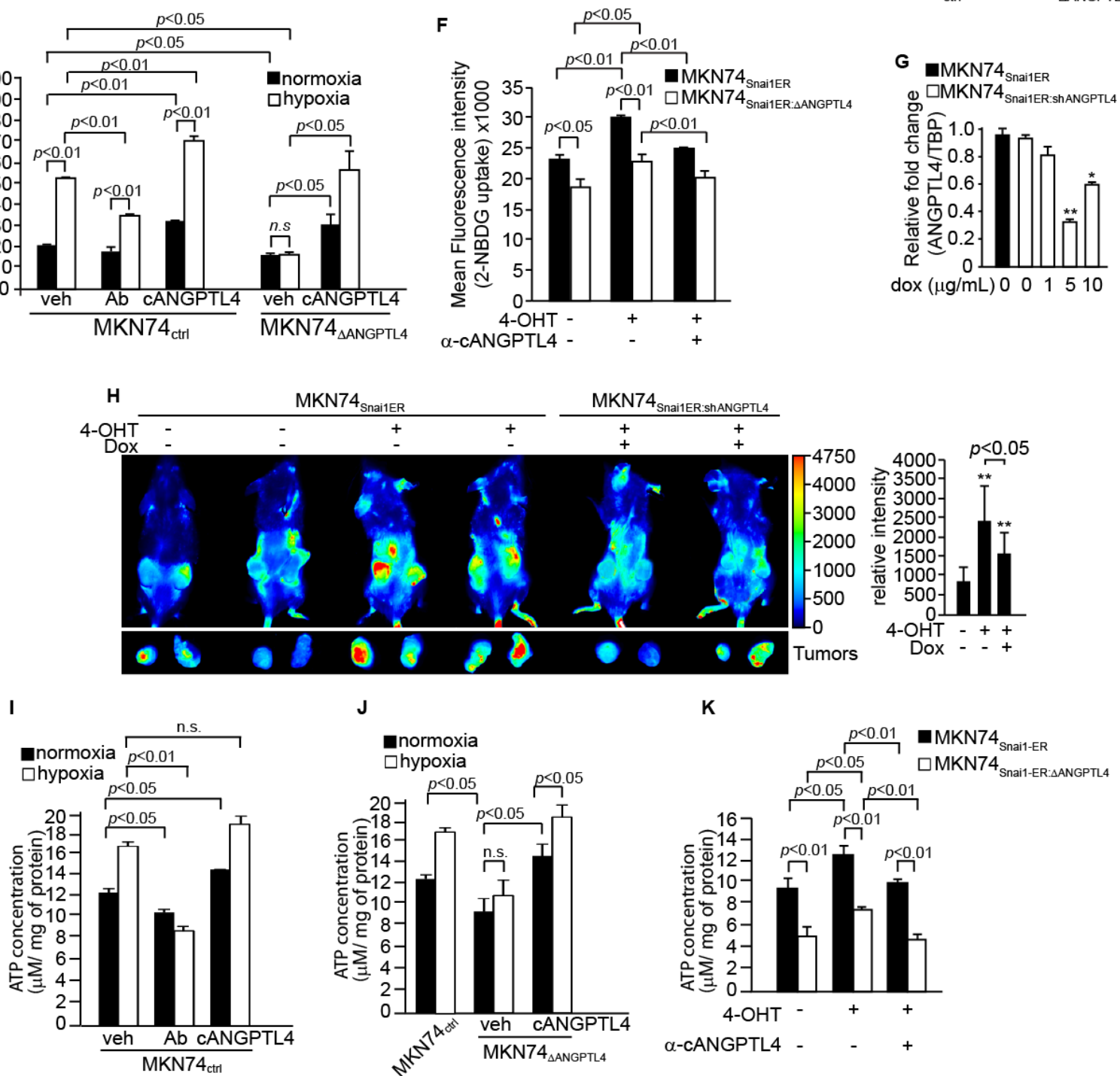


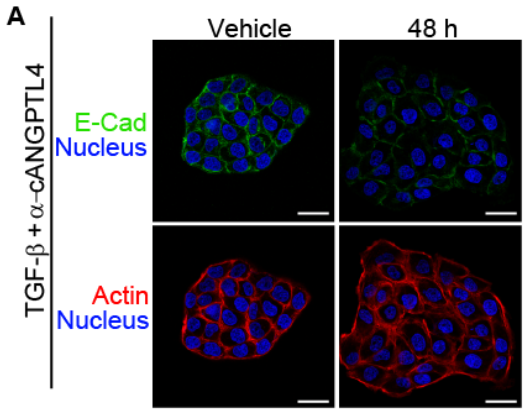

B

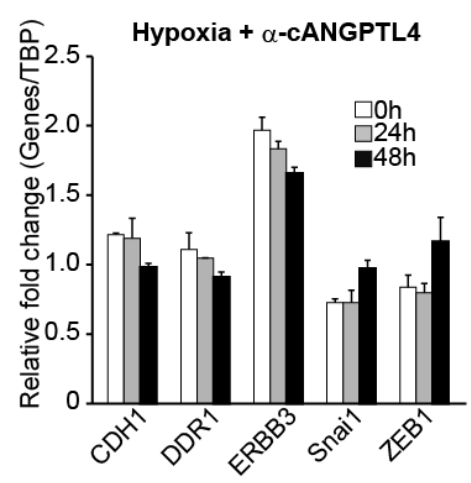

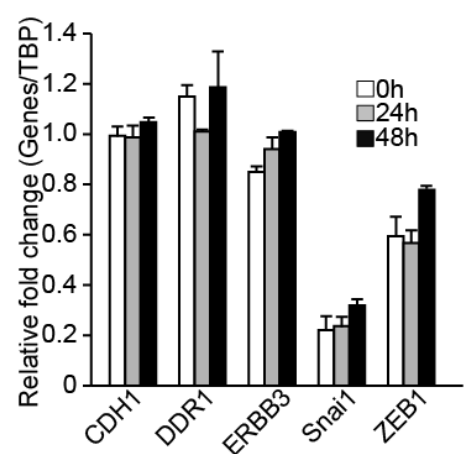

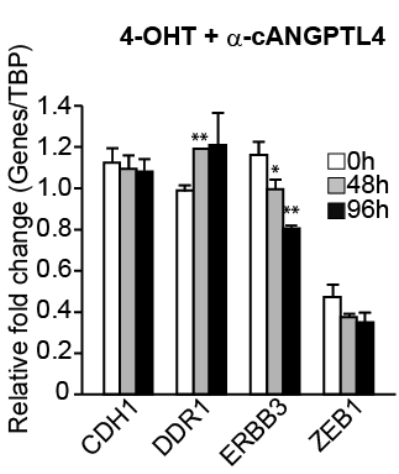

$c$

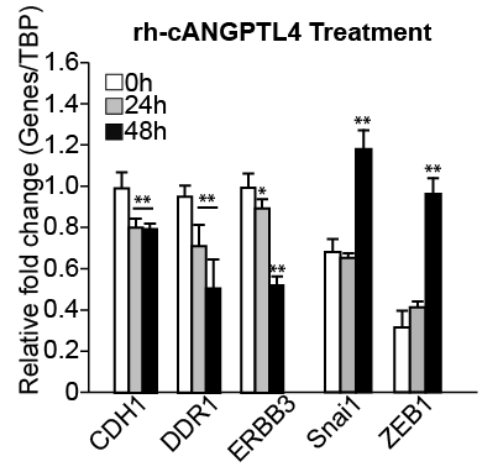

D $\quad \mathrm{MCF}_{\text {CTRL }}$ MCF7 $7_{\triangle A N G P T L 4}$ Hypoxia Oh 48h $0 \mathrm{~h} \mathrm{48h} \mathrm{KDa}$ E-cad - - - 140

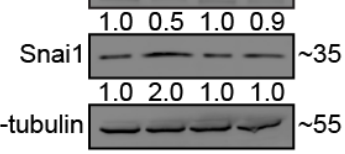
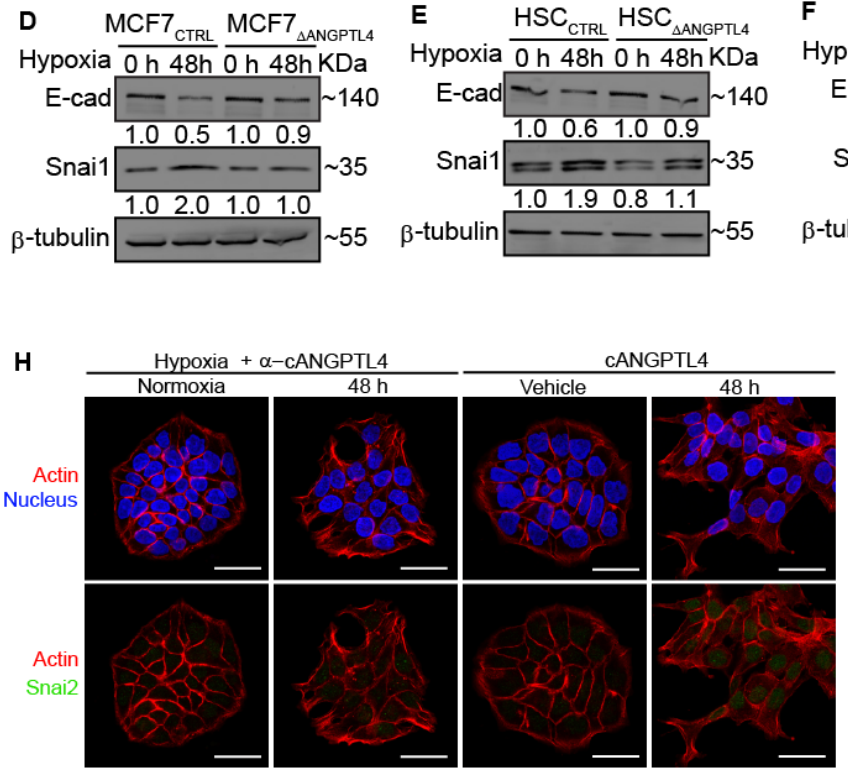
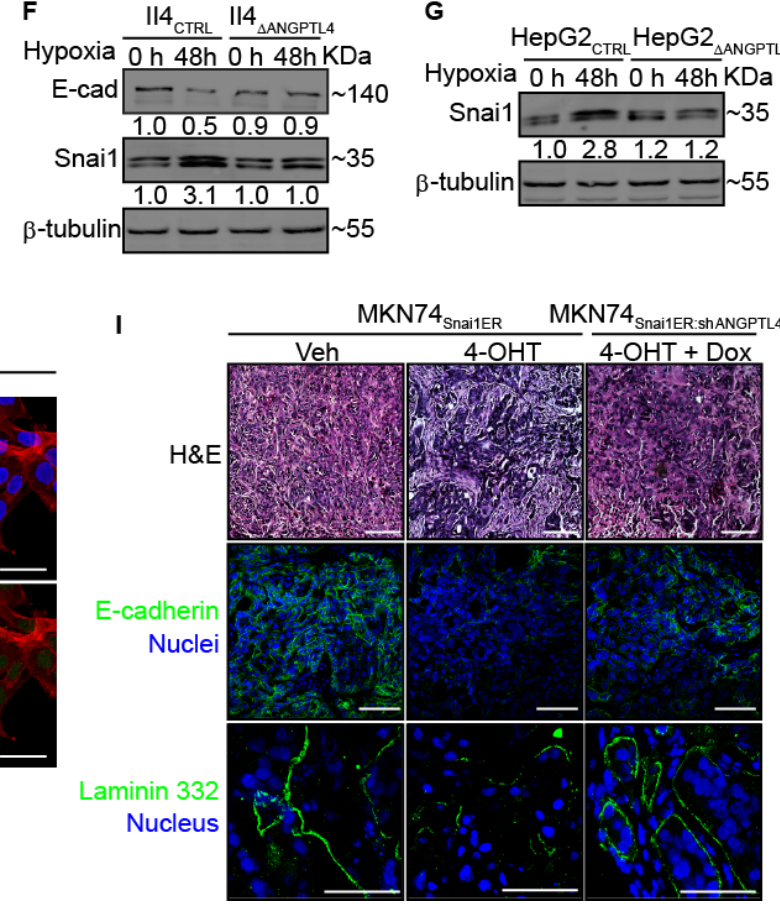

G

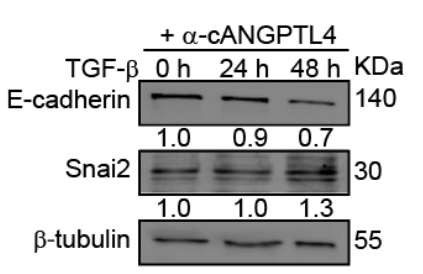

HepG2 ${ }_{\text {CTRL }}$ HepG2 $2_{\triangle A N G P T L 4}$ Hypoxia 0 h 48h $0 \mathrm{~h} \mathrm{48h} \mathrm{KDa}$ Snai1 $=\approx=20=0$ $\beta$-tubulin $\begin{array}{lll}1.0 & 2.81 .2 & 1.2 \\ - & - & -\end{array}$ 
A

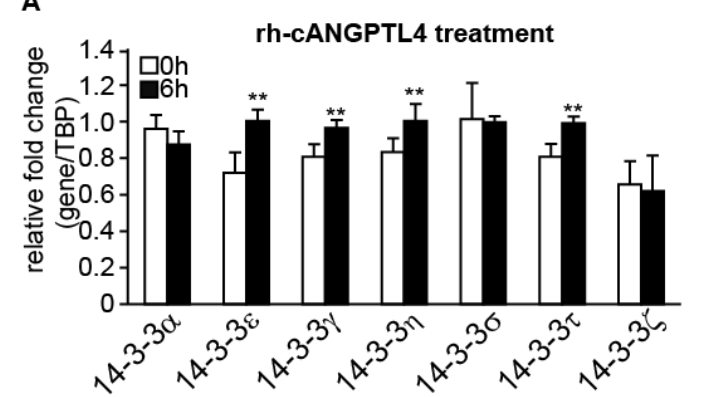

B $\quad \mathrm{MCF}_{\text {CTRL }} \mathrm{MCF} 7_{\triangle \mathrm{ANGPTL4}}$ Hypoxia $0 \mathrm{~h} \mathrm{48h} 0 \mathrm{~h} \mathrm{48h}$ KDa p-AKT - - - 70 (S473) $\begin{array}{llll}1.0 & 1.8 & 0.9 & 1.2\end{array}$

$\mathrm{AKT}----70$

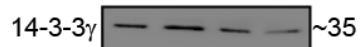
$\begin{array}{llll}1.0 & 2.0 & 1.0 & 0.7\end{array}$ c $\quad \mathrm{Il} 4_{\mathrm{CTRL}} \mathrm{II} 4_{\triangle \mathrm{ANGPTL}}$

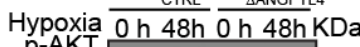
$\begin{array}{llll}\text { (S473) } & - & - & - \\ 1.0 & 3.4 & 1.0 & 1.6\end{array}$ $\mathrm{AKT}----70$

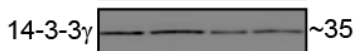

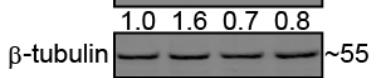

$\beta$-tubulin - - $\sim 55$

D HSC $_{\text {CTRL }}$ HSC $_{\triangle A N G P T L 4}$ Hypoxia $\overline{\mathrm{Oh} \mathrm{48h}} \overline{\mathrm{Oh} \mathrm{48h} \mathrm{KDa}}$ $\mathrm{p}-\mathrm{AKT} \longrightarrow-\mathrm{Z}$
(S473) (S473) $\begin{array}{lllll}1.0 & 2.8 & 1.0 & 1.6 \\ \text { AKT }- & - & - & - & -\end{array} 70$

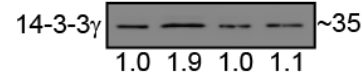
$\beta$-tubulin $\square-\square-\square 5$
E HepG2 ${ }_{\text {ctRL }}$ HepG2 ${ }_{\triangle A N G P T L 4}$

Hypoxia $\overline{\mathrm{Oh} \mathrm{48h}} \overline{\mathrm{Oh} \mathrm{48h}} \mathrm{KDa}$

p-AKT $-\cdots-\cdots 70$

(S473) \begin{tabular}{lllll}
\hline & & & & \\
1.0 & 2.1 & 0.7 & 1.0
\end{tabular}

$\mathrm{AKT}--\ldots-10$

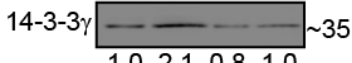

$\beta$-tubulin $--\square 5$ 


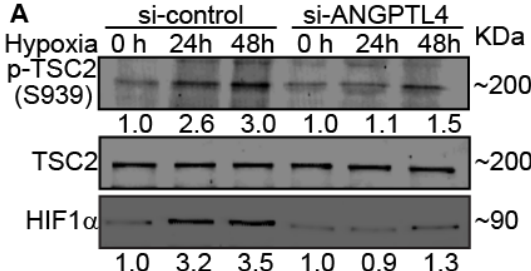

B 4-OHT $\overline{\mathrm{Oh} 48 \mathrm{~h} 96 \mathrm{~h}} \mathrm{KD}$ p-TSC2 $-\div-\div 200$ (S939)

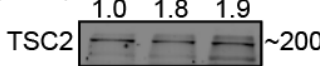

c $\quad$ MKN74

cANGPTL $4 \longdiv { 0 \mathrm { h } 2 4 \mathrm { h } 4 8 \mathrm { h } } \mathrm { KDa }$

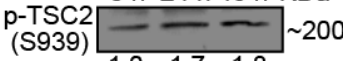

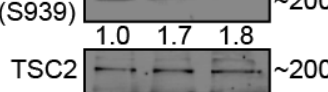

D

IP: Snai1

si-control si-ANGPTL4

Hypoxia exposure $0 \mathrm{~h} \quad 48 \mathrm{~h} \quad \mathrm{~h} \quad 48 \mathrm{~h} \mathrm{KDa}$

IB:Snai1 $\sim 30$

IB:14-3-3y

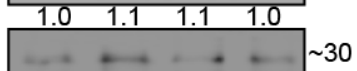

IB: $14-3-3 \eta$

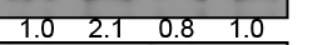

IB: $14-3-3 \tau$ $\begin{array}{llll}1.0 & 1.1 & 1.1 & 1.0\end{array}$

IB:14-3-3

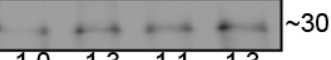
$\sim 30$

E

14-3-3y \& TSC2 ${ }_{S 939}$

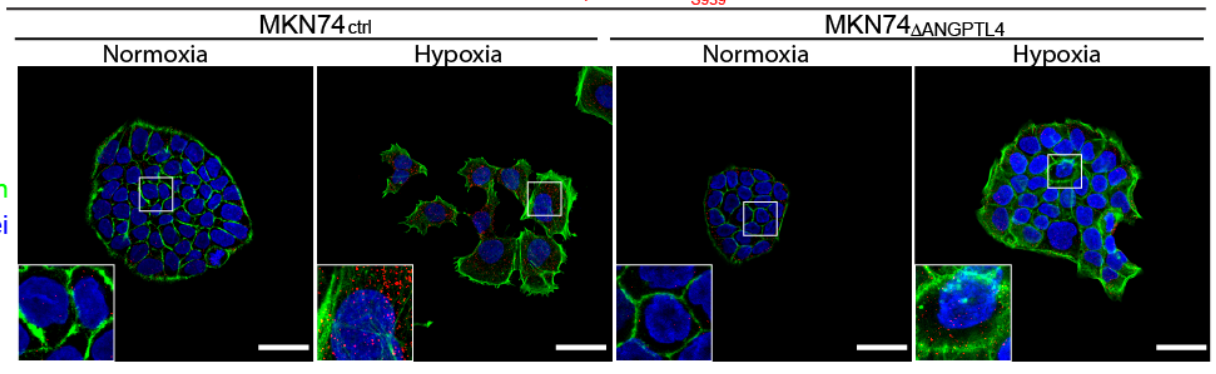

F

14-3-3y \& Snai 1

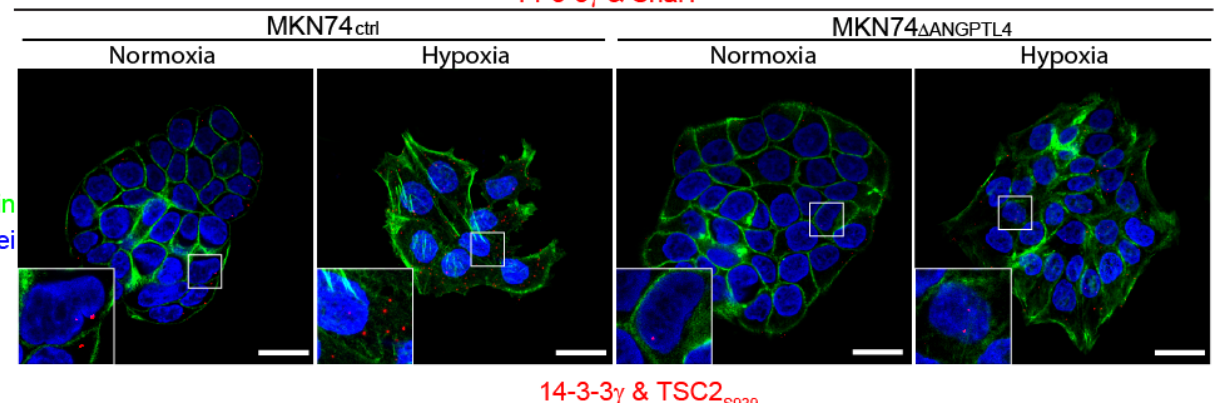

G

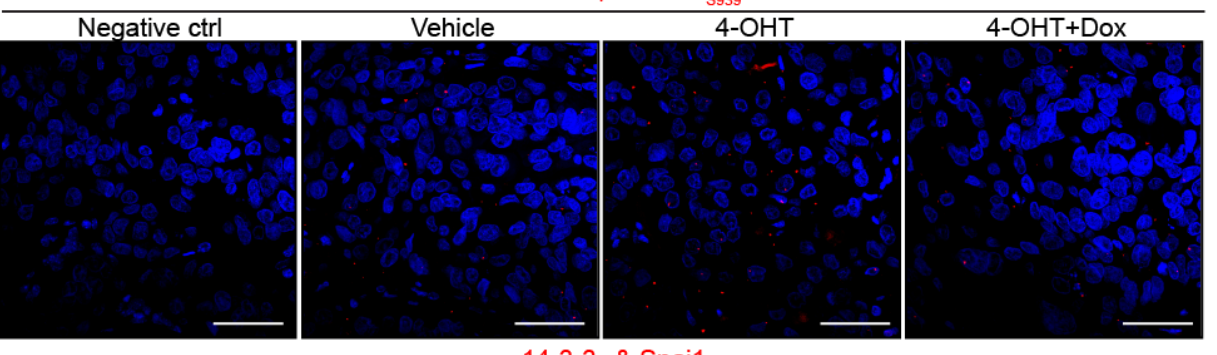

$\mathrm{H}$

14-3-3y \& Snai1

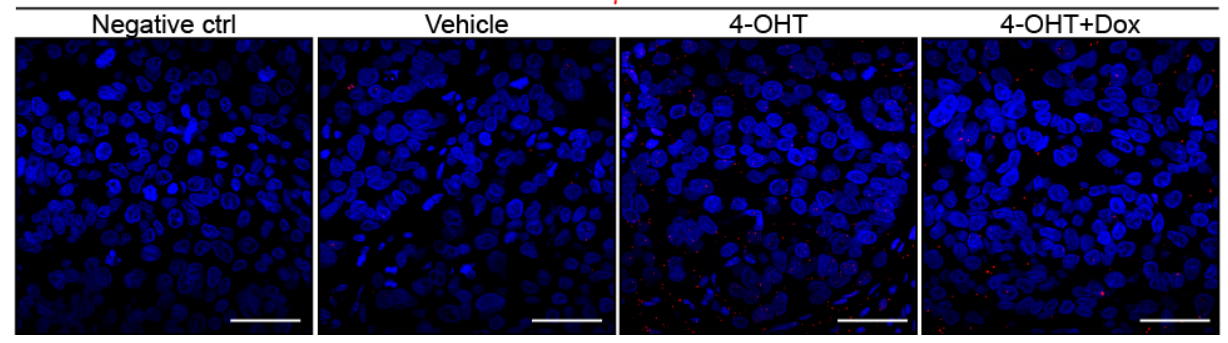




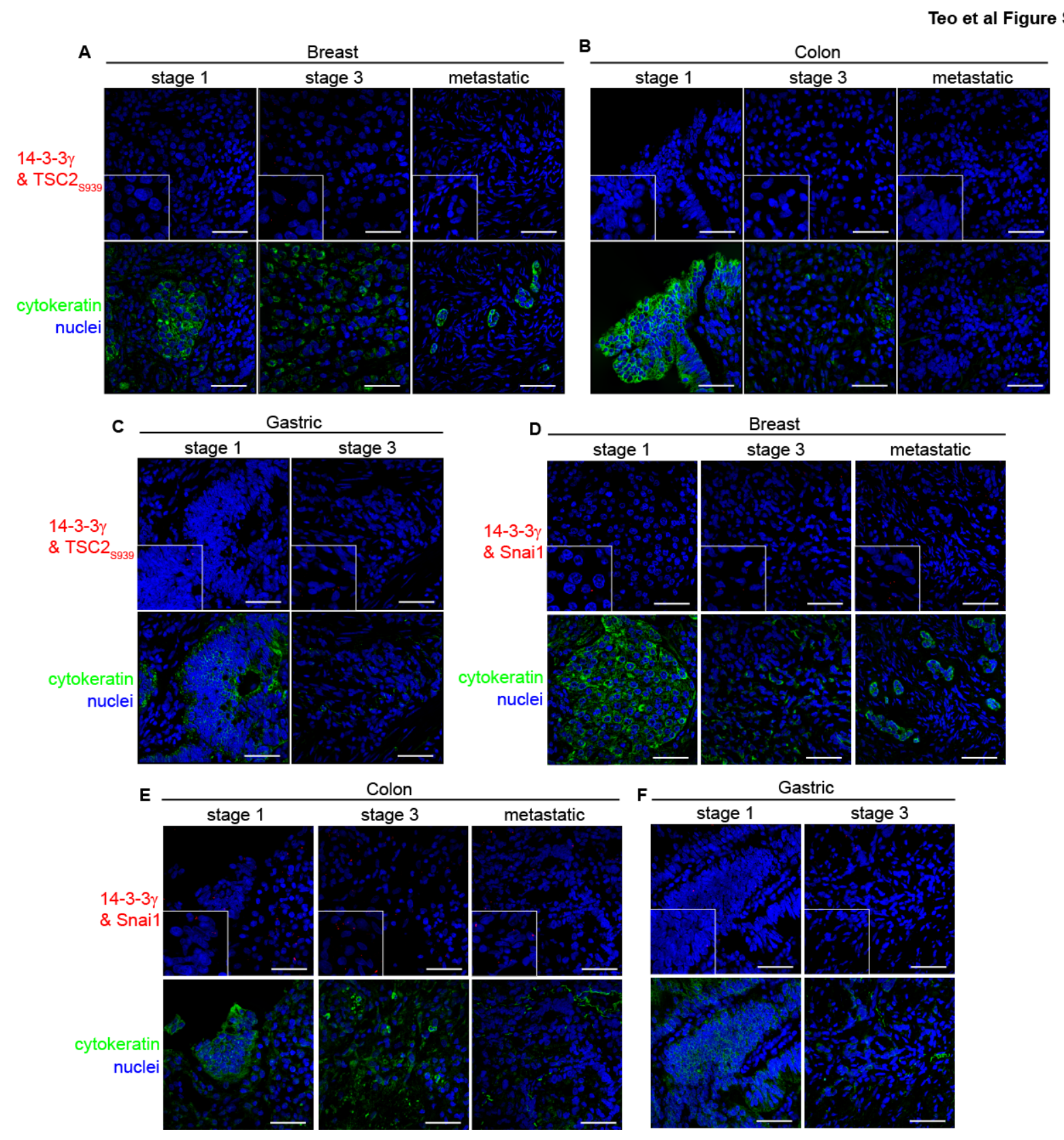




\section{Supplementary Figure Legends}

Figure S1, related to Figure 1. (A, C) Immunofluorescence staining of E-cadherin in TGF$\beta$-treated MKN74 cells (A) or Snai2 in hypoxia-treated MKN74 cells (C) at indicated time intervals. Cells were counterstained with DAPI (blue) for nuclei and phalloidin (red) for actin cytoskeleton. Scale bar $=40 \mu \mathrm{m}$. (A, right panel) Immunodetection and relative gene expression of EMT markers in MKN74 cells exposed to TGF- $\beta$ at indicated time intervals. Data (mean \pm SD) from three independent experiments. * $P<0.05$, ** $P<0.01$. Immunodetection and relative gene expression of Snai1 in MKN74 $4_{\text {CTRL }}$ or MKN74 $4_{\text {Snai1ER }}$ cells. (D) Cellular ATP measurement in MKN74 CTRL $_{\text {and }}$ MKN74 $4_{\text {Snai1ER }}$ cells based on bioluminescence after the indicated treatments. The ATP concentration was determined from a standard curve using pure ATP. For immunoblots, loading controls are from the same sample.

Figure S2, related to Figures 2. (A) Immunodetection of dominant-negative (dn) or constitutively active (ca) AKT and AMPK in MKN74 cells after transfection (n=3). (B) Immunodetection of E-cadherin and Snai1 in hypoxia-treated MKN74 cells harboring the respective modifications. (C) Representative macroscopic and H\&E images of tumors from MKN74 $4_{\text {Snai1ER }}$ tumor sections exposed to the indicated treatments. Scale bar $=40 \mu \mathrm{m}$ (D) Focus array of critical ABC transporters' genes involved in drug resistance across the three EMT models over the indicated time points. Green: down-regulated; Red: up-regulated. For immunoblots, loading controls are from the same sample.

Figure S3, related to Figures 3. (A, C, G) Relative gene expression of ANGPTL4 in MKN74 $_{\text {CTRL }}$, MKN74 $_{\triangle \mathrm{ANGPTL} 4}$, MKN74 $_{\text {Snai1ER }}$ or MKN74 $4_{\text {Snai1ER:shANGPTL4 }}$ cells after indicated treatments. (B) Immunodetection of selected proteins involved in the autophagy in MKN74 $_{\mathrm{CTRL}}, \mathrm{MKN74}_{\triangle \mathrm{ANGPTL} 4}$ and MKN74 $4_{\text {Snai1ER }}$ cells. (D, F) FACS analysis of fluorescent glucose analog, 2-NBDG, uptake in MKN74 CTRL, MKN74 $_{\triangle \mathrm{ANGPTL} 4}, \mathrm{MKN74} 4_{\text {Snai1ER, or }}$ 
MKN74 $_{\text {SnailER:AANGPTL4 }}$ cells after the indicated treatments. (E) Glycolysis rate in MKN74 $_{\text {CTRL }}$ and MKN74 ${ }_{\triangle A N G P T L 4}$ cells after the indicated treatments. (H) In vivo 2-DG fluorescence imaging of mice bearing tumors that received the indicated treatments. (I-K) Cellular ATP measurement in MKN74 $4_{\text {CTRL }}$ MKN74 ${ }_{\triangle A N G P T L 4}$, MKN74 $4_{\text {Snai1ER, }}$ or MKN74 $_{\text {SnailER:AANGPTL4 }}$ cells based on bioluminescence after the indicated treatments. The ATP concentration was determined from a standard curve using pure ATP. veh: vehicle; Ab: $\alpha$-cANGPTL4.

Figure S4, related to Figure 4. (A, H) Immunofluorescence staining of E-cadherin in TGF$\beta$ - treated MKN74 cells (A) or Snai2 in hypoxia-treated MKN74 cells in the presence of antibodies against cANGPTL4 and rh-cANGPTL4-treated MKN74 cells $(\mathbf{H})$ at the indicated time intervals. Cells were counterstained with DAPI (blue) for nuclei and phalloidin (red) for actin cytoskeleton. Scale bar $=40 \mu \mathrm{m}$. (B, C) Relative expression of EMT markers in hypoxia-exposed MKN74, 4-OHT-treated MKN74 $4_{\text {Snai1ER }}$ cells (B) or rh-cANGPTL4-treated MKN74 cells (C) exposed to $\alpha$-CANGPTL4 at the indicated time intervals. Data (mean \pm SD) from three independent experiments. ${ }^{*} P<0.05$, $* * P<0.01$. (D-G) Immunodetection of indicated proteins in hypoxia-treated, wild-type or ANGPTL4-knockdown MCF7 (D), HSC (E), II4 (F) and HepG2 cells (G). Loading controls are from the same sample. (I) Representative H\&E images and immunofluorescence images of E-cadherin and laminin 332 from MKN74 $4_{\text {Snai1ER:shANGPTL4 }}$ tumor sections exposed to the indicated treatments. Scale bar $=$ $40 \mu \mathrm{m}$ (top) or $100 \mu \mathrm{m}$ (bottom). For immunoblots, loading controls are from the same sample.

Figure S5, related to Figure 5. (A) Relative expression of various 14-3-3 isoforms in MKN74 cells in the presence of rh-cANGPTL4 after 6 hours. Data (mean \pm SD) from three independent experiments. * $P<0.05$, ** $P<0.01$. (B-E) Immunodetection of indicated 
proteins in hypoxia-treated MCF7 (B), II4 (C), HSC (D) and HepG2 cells (E) at the indicated time points with or without ANGPTL4. Loading controls are from the same sample.

Figure S6, related to Figure 6. (A-D) Immunodetection of the indicated proteins in hypoxiatreated MKN74 CTRL $_{\text {or MKN74 }}$ ANGPTL4 $_{4}$ cells (A), 4-OHT-treated MKN74 $4_{\text {Snai1ER }}$ cells (B), rh-

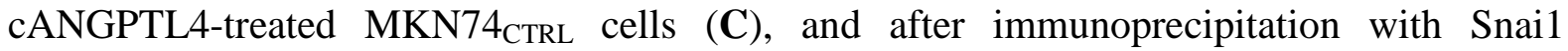
antibody (D) at the indicated time points. Loading controls are from the same sample. (E-H)

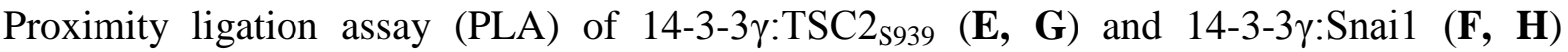

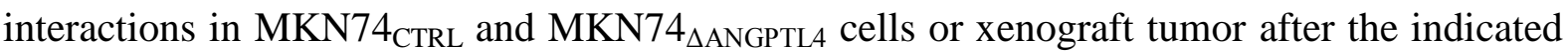
treatments. PLA signals are in red. MKN74 and xenograft tumor cells were counterstained with DAPI (blue) for nuclei and alexa-488 phalloidin (green) for actin cytoskeleton. Scale bar $=40 \mu \mathrm{m}(\mathbf{E}, \mathbf{G})$ or $50 \mu \mathrm{m}(\mathbf{F}, \mathbf{H})$. Data (mean \pm SD) from three independent experiments. * $P<0.05, * * P<0.01$.

Figure S7, related to Figure 7. (A-F) Proximity ligation assay (PLA) of 14-3-3 $\gamma: \mathrm{TSC} 2_{\text {S939 }}$ (A-C) and 14-3-3y:Snail (D-F) interactions in patient biopsies with the indicated tumor grades. PLA signals are in red. Tumor cells were counterstained with DAPI (blue) for nuclei and pan-cyotkeratin (green) to differentiate epithelial and mesenchymal origin. Scale bar = $50 \mu \mathrm{m}$. Data (mean \pm SD) from three independent experiments. $* P<0.05, * * P<0.01$. 


\section{Supplementary Experimental Procedures}

Retrovirus transduction. MKN74 $4_{\text {Snai1ER }}$ cells were generated using retroviral transduction as previously described (Mani et al., 2008). Briefly, HEK 293T cells were transfected with pCL10A1 retrovirus packaging vector (Imgenex, USA) and pWZL-Snai1-ER (Addgene, USA). Harvested pseudoviruses were used to infect MKN74 cells. Following transduction, cells were selected with $5 \mu \mathrm{g} / \mathrm{mL}$ of blasticidin hydrochloride (Sigma Aldrich, USA).

Generation of dual-inducible MKN74 $_{\text {Snai1ER/shANGPTL4 }}$ cell line. Doxycycline-inducible pSingle-tTS-shRNA vector carrying either shANGPTL4 or scrambled shRNA was introduced into MKN74 $4_{\text {Snai1ER }}$ cells using Fugene HD reagent as per manufacturer protocol (Promega, USA). MKN74 Snai1ER/shANGPTL4 $_{\text {and MKN74 }}$ Snai1ER/shscrambled cells were selected with $1 \mathrm{mg} / \mathrm{mL}$ of G418 (Sigma Aldrich, USA). The efficiency of knockdown was verified by qPCR and immunoblotting after treatment with various concentrations of doxycycline (up to $10 \mu \mathrm{g} / \mathrm{mL}$ ).

Expression and purification of recombinant cANGPTL4 proteins. The expression and purification of recombinant cANGPTL4 were performed as previously described (Goh et al., 2010).

Microarray analysis. Cells were seeded at a density of $5.25 \times 10^{2} \mathrm{cell} / \mathrm{cm}^{2}$ to allow the formation of individual colonies before indicated treatments. Hypoxia treatments were performed in hypoxic chamber (Stem cell Technology, USA) purged with 5\% $\mathrm{CO}_{2}$ and $95 \%$ $\mathrm{N}_{2}$ to obtain $\mathrm{O}_{2}$ concentration at $1 \%$ as determined by an $\mathrm{O}_{2}$ sensor. For TGF- $\beta$-induced EMT, cells were treated with $10 \mathrm{ng} / \mathrm{mL}$ of TGF- $\beta$ for 2 days. For Snail-mediated EMT, MKN74 $_{\text {Snai1ER/shANGPTL4 }}$ and MKN74 $4_{\text {Snai1ER }}$ cells were exposed to $20 \mathrm{ng} / \mathrm{mL}$ of 4hydroxytamoxifen (4-OHT) for 4 days. RNA was extracted from the respective treatments with Trizol following the manufacturer's protocol. Further sample processing of the RNA 
was carried out using Applause ${ }^{\circledR}$ WT-Amp ST System (NuGEN), and microarray experiments were performed on GeneChip ${ }^{\circledR}$ Human Gene 1.0 ST arrays according to the manufacturer’s instructions.

Immunofluorescence staining. EMT was identified by immunofluorescence staining for Ecadherin and Snai2. MKN74 cells were fixed with 4\% paraformaldehyde for 10 min, permeablized with $0.2 \%$ Triton-X 100 for 10 min and blocked with $3 \%$ normal goat serum (NGS) containing $0.05 \%$ Triton-X 100 in a humidified chamber for $1 \mathrm{~h}$ at room temperature. Following three washes in PBS, cells were incubated overnight at $4{ }^{\circ} \mathrm{C}$ with either antihuman-E-cadherin (1:200) or Snai1 (1:100) antibodies in 3\% NGS. After three washes, cells were incubated for $1 \mathrm{~h}$ at room temperature with Alexa488-secondary antibodies (1:250) and counterstained with Alexa594-phalloidin for F-actin and DAPI for nuclei. Immunostainings performed without primary antibodies served as negative controls. Images were acquired using Zeiss LSM710 confocal microscope with a Plan-APOCHROMAT 40x/1.4 oil objective and ZEN2009 software.

Proximity Ligation Assay (PLA) and analysis. MKN74 $4_{C T R L}$ or MKN74 ${ }_{\triangle A N G P T L}\left(5 \times 10^{3}\right.$ cells) were subjected to either normoxia or hypoxia for 2 days at $37{ }^{\circ} \mathrm{C}$. At indicated time, MKN74 cells were fixed, permeabilized and blocked similar to immunofluorescence staining. Next, MKN74 cells were incubated with monoclonal mouse-anti human 14-3-3 $\gamma$ antibody (1:200) and monoclonal rabbit anti-human Snai1 antibody (1:200) in 3\% NGS overnight at 4 ${ }^{\circ} \mathrm{C}$. PLA was carried out as per manufacturer's protocol (Olink Bioscience, USA). Images were taken using Carl Zeiss confocal microscope LSM710 using a Plan-APOCHROMAT 63x/1.4 oil DIC objective, and ZEN2012 LE software with constant exposure and gain. Number of protein interaction was quantified using the "BlobFinder" (Allalou and Wählby, 2009). 
Immunoprecipitation and immunoblot. For immunoprecipitation, at the indicated time points after treatments, cells were lysed using IP lysis buffer (25mM Tris-HCl pH7.4, 150mM NaCl, 1\% NP-40, 1mM EDTA). The lysate was then incubated with indicated antibodies overnight at $4{ }^{\circ} \mathrm{C}$ with constant rotation. Following that, the antibodies were affinity precipitated using protein A/G beads (Santa Cruz, USA). Proteins were released by boiling for 10 min in Lamelli's buffer. Protein extracts were resolved using $12 \%$ SDSpolyacrylamide gel electrophoresis and electrotransferred (25 mM Tris, $192 \mathrm{mM}$ glycine, 20\% methanol) onto a low fluorescence PVDF membrane for immunoblot analysis. Membranes were stripped and re-probed for other proteins as described (Yeung and Stanley, 2009). Protein bands were detected using Odyssey CLx Infrared Imaging system (LI-COR, USA).

Energy charge determination. Cellular levels of AMP, ADP and ATP were determined by HPLC. A Shimadzu LC-20AD series Quaternary Gradient HPLC system with PDA detector using a Hypersil ODS 150 mm x 4.6 mm I.D. C18 reverse-phase column with $3 \mu \mathrm{m}$ particle size and $5 \mu \mathrm{m}$ Hypersil ODS C18 guard column (Thermo Scientific, USA) was used. Nucleotides were extracted as previously described (44). Nucleotides standards were from Sigma Aldrich, USA. Separation of the nucleotides were carried out with a mobile phase containing $60 \mathrm{mmol} / \mathrm{L}$ of $\mathrm{KH}_{2} \mathrm{PO}_{4}, 0.45 \mathrm{mmol} / \mathrm{L}$ of tetrabutylammonium bromide and 1.26 mol/L of acetonitrile. The $\mathrm{pH}$ of the mobile phase was adjusted to 3.20 using concentrated phosphoric acid. Retention times of the individual nucleotides were matched to that of the respective nucleotide standards. Areas under the peaks for the respective nucleotides were tabulated and energy charge was calculated using the formula: Energy Charge = $[\mathrm{ATP}]+1 / 2[\mathrm{ADP}] /[\mathrm{ATP}]+[\mathrm{ADP}]+[\mathrm{AMP}]$ 
ATP quantification. ATP level in the cancer cells were quantified using the ATP determination assay kit (Invitrogen, USA) as per manufacturer protocols. Briefly, ATP was released from the cells using somatic cell lysis buffer (Sigma Aldrich, USA) after treatment for indicated time period. ATP levels were assayed and the luminescence readings were taken using a GloMax 20/20 luminometer (Promega, USA). Luminescence readings were plotted against an ATP standard curve to obtain the actual ATP concentration followed by normalization to the protein concentration.

Glucose uptake assay. Briefly, cells were incubated with $10 \mu \mathrm{M}$ of 2-NBDG for $12 \mathrm{~h}$. The cells were then harvested and subjected to FACS analysis using BD Accuri C6 flow cytometer (BD Biosciences, USA). Data was analysed using FlowJo and plotted based on mean intensity.

Metabolic analysis. Oxygen consumption rate (OCR) and extracellular acidification rate (ECAR) were measured in real-time using a XF96 flux analyzer (Seahorse Bioscience, USA). Cells were seeded on 96-well microplates. All measurements were normalized against total protein content from cell lysates.

Drug efflux assay. The activities of the multidrug resistance proteins MDR1, MRP and BCRP were measured using eFluxx-ID Green Multidrug Resistance Assay Kit (ENZ-51029K100, Enzo Lifesciences, Farmingdale, NY) according to the manufacturer's instructions. Briefly, single cell suspensions were harvested and equal numbers of cells ( $2 \times 10^{5} /$ condition) were resuspended in full media containing either DMSO or specific inhibitors (Verapamil: MDR1 inhibitor; MK-571: MRP1 inhibitor; Novobiocin: BCRP inhibitor; $200 \mu \mathrm{m}$ ) and incubated for $10 \mathrm{~min}$ at $37^{\circ} \mathrm{C}$. EFLUXX-ID ${ }^{\circledR}$ Green Detection Reagent was then added and incubated at $37^{\circ} \mathrm{C}$ for 40 min before FACS analysis using BD Accuri C6 flow cytometer (BD Biosciences, USA). Data was analysed using FlowJo and plotted based on mean intensity. 
Kinase inhibitor array. MKN74 were treated with 95 different kinase inhibitors (SYN2103; Synkinase, Australia) in the absence and presence of rh-cANGPTL4 (10 $\mu \mathrm{g} / \mathrm{mL})$ for 6 h. RNA was isolated and reverse-transcribed using the iScript Reverse Transcription Supermix. qPCR was done using the $\mathrm{KAPA}^{\mathrm{TM}}$ SYBR qPCR Universal Master Mix (KAPABiosystems).

Table S1. ChIP Primer sequences.

\begin{tabular}{|l|l|l|}
\hline & Forward (5' -> 3') & Reverse (5’ -> 3’) \\
\hline $\begin{array}{l}\text { Snai1- } \\
\text { binding } \\
\text { region }\end{array}$ & TAGAGGGTCACCGCGTCTAT & GGCTGGAGTCTGAACTGACTTC \\
\hline $\begin{array}{l}\text { Negative } \\
\text { Control }\end{array}$ & TCTAGGAGCAGCAGGAAGGGAG & GTCCCTTTCATCTCCTGGCAAG \\
\hline
\end{tabular}


Table S2.Real-time PCR Primers

\begin{tabular}{|c|c|c|}
\hline Genes & Forward Sequence (5' to 3') & Reverse Sequence (3’ to 5’) \\
\hline ANGPTL4 & TCCAACGCCACCCACTTAC & TGAAGTCATCTCACAGTTGACCA \\
\hline CDH1 & GCCGAGAGCTACACGTTCA & GACCGGTGCAATCTTCAAA \\
\hline DDR1 & CACTCCGCTCCCTGTGTC & AGAGGTGCGACTGGAACAA \\
\hline ERBB3 & CTGATCACCGGCCTCAAT & GGAAGACATTGAGCTTCTCTGG \\
\hline Snai1 & GCCTTCAACTGCAAATACTGC & CTTCTTGACATCTGAGTGGGTC \\
\hline TBP & GCTGGTTATCGGGAGTTGG & ACTGGCCTGGTGTCCTAGAG \\
\hline ZEB1 & GCCAACAGACCAGACAGTGTT & TCTTGCССТTССТTTTССТG \\
\hline $14-3-3 \alpha / \beta$ & GGCAAAGAGTACCGTGAGAAG & CTGGTTGTGTAGCATTGGGAATA \\
\hline $14-3-3 \gamma$ & AGCCACTGTCGAATGAGGAAC & CTGCTCAATGCTACTGATGACC \\
\hline $14-3-3 \varepsilon$ & GATTCGGGAATATCGGCAAATGG & GCTGGAATGAGGTGTTTGTCC \\
\hline $14-3-3 \eta$ & TGGCTGATGGAAACGAAAAGAA & CCTCTGCTAAGTAGCGGTAGT \\
\hline $14-3-3 \sigma$ & TGACGACAAGAAGCGCATCAT & GTAGTGGAAGACGGAAAAGTTCA \\
\hline $14-3-3 \tau$ & AGGGTCATCTCTAGCATCGAG & CСАCTTTCTCCCGATAGTCCTT \\
\hline $14-3-3 \zeta$ & CCTGCATGAAGTCTGTAACTGAG & GACCTACGGGCTCCTACAACA \\
\hline
\end{tabular}


Table S3. Human tumor biopsies details

\begin{tabular}{|c|c|c|c|c|c|c|c|}
\hline $\begin{array}{l}\text { Sample } \\
\text { Source }\end{array}$ & Sex & Age & Diagnosis & $\begin{array}{c}\text { Histological } \\
\text { diagnosis }\end{array}$ & Grade & TNM & Stage \\
\hline Breast & $\mathrm{F}$ & 51 & cancer & $\begin{array}{l}\text { infiltrating ductal } \\
\text { carcinoma }\end{array}$ & G2 & T1cN0M0 & I \\
\hline Breast & $\mathrm{F}$ & 62 & cancer & $\begin{array}{c}\text { infiltrating } \\
\text { lobular carcinoma }\end{array}$ & G2 & T2N3aM0 & IIIC \\
\hline $\begin{array}{c}\text { Breast to } \\
\text { ovary }\end{array}$ & $\mathrm{F}$ & 59 & metastases & $\begin{array}{l}\text { infiltrating ductal } \\
\text { carcinoma }\end{array}$ & N/A & recurrent & \\
\hline Colon & $\mathrm{M}$ & 66 & cancer & adenocarcinoma & G1 & T2N0M0 & $\mathrm{I}$ \\
\hline Colon & $\mathrm{M}$ & 80 & cancer & adenocarcinoma & G1 & T3N1M0 & IIIB \\
\hline $\begin{array}{l}\text { Colon to } \\
\text { lung }\end{array}$ & $\mathrm{F}$ & 42 & metastases & adenocarcinoma & N/A & recurrent & \\
\hline Stomach & $\mathrm{M}$ & 64 & cancer & adenocarcinoma & G2 & T2N0M0 & IB \\
\hline Stomach & $\mathrm{M}$ & 58 & cancer & adenocarcinoma & G3 & T3N2M0 & IIIA \\
\hline Tongue & M & 64 & cancer & $\begin{array}{l}\text { squamous cell } \\
\text { carcinoma }\end{array}$ & G2 & T2N1M0 & III \\
\hline Tongue & M & 58 & cancer & $\begin{array}{l}\text { squamous cell } \\
\text { carcinoma }\end{array}$ & G1 & T2N0M0 & II \\
\hline
\end{tabular}

\section{Supplemental References}

Allalou, A., and Wählby, C. (2009). BlobFinder, a tool for fluorescence microscopy image cytometry. Comput Methods Programs Biomed 94, 58-65.

Goh, Y.Y., Pal, M., Chong, H.C., Zhu, P., Tan, M.J., Punugu, L., Tan, C.K., Huang, R.L., Sze, S.K., and Tang, M.B.Y., et al. (2010). Angiopoietin-like 4 interacts with matrix proteins to modulate wound healing. J Biol Chem 285, 32999-33009.

Yeung, Y.G., and Stanley, E.R. (2009). A solution for stripping antibodies from polyvinylidene fluoride immunoblots for multiple reprobing. Anal Biochem 389, 89-91. 